\title{
Bimodal imaging of molecular targets associated with plaque vulnerability
}

Citation for published version (APA):

Prinzen, L. (2009). Bimodal imaging of molecular targets associated with plaque vulnerability. [Doctoral Thesis, Maastricht University]. Datawyse / Universitaire Pers Maastricht.

https://doi.org/10.26481/dis.20090917lp

Document status and date:

Published: 01/01/2009

DOI:

10.26481/dis.20090917/p

Document Version:

Publisher's PDF, also known as Version of record

\section{Please check the document version of this publication:}

- A submitted manuscript is the version of the article upon submission and before peer-review. There can be important differences between the submitted version and the official published version of record.

People interested in the research are advised to contact the author for the final version of the publication, or visit the DOI to the publisher's website.

- The final author version and the galley proof are versions of the publication after peer review.

- The final published version features the final layout of the paper including the volume, issue and page numbers.

Link to publication

\footnotetext{
General rights rights.

- You may freely distribute the URL identifying the publication in the public portal. please follow below link for the End User Agreement:

www.umlib.nl/taverne-license

Take down policy

If you believe that this document breaches copyright please contact us at:

repository@maastrichtuniversity.nl

providing details and we will investigate your claim.
}

Copyright and moral rights for the publications made accessible in the public portal are retained by the authors and/or other copyright owners and it is a condition of accessing publications that users recognise and abide by the legal requirements associated with these

- Users may download and print one copy of any publication from the public portal for the purpose of private study or research.

- You may not further distribute the material or use it for any profit-making activity or commercial gain

If the publication is distributed under the terms of Article $25 \mathrm{fa}$ of the Dutch Copyright Act, indicated by the "Taverne" license above, 
Bimodal imaging of molecular targets associated with plaque vulnerability 
ISBN 978-90-5278-847-0

(C) Lenneke Prinzen, Maastricht 2009

Printing: Datawyse / Universitaire Pers Maastricht

Cover image is a canyon in Zion National park, Utah, USA.

Cover design by Bart Prinzen. 


\title{
Bimodal imaging of molecular targets associated with plaque vulnerability
}

\author{
PROEFSCHRIFT
}

ter verkrijging van de graad van doctor aan de Universiteit Maastricht, op gezag van de Rector Magnificus, prof. mr. G.P.M.F. Mols

volgens het besluit van het College van Decanen,

in het openbaar te verdedigen

op donderdag 17 september 2009 om 16 uur.

door

Lenneke Prinzen 
Promotores:

Prof. dr. D.W. Slaaf

Prof. dr. M.A.M.J. van Zandvoort (RWTH, Aachen)

Prof. dr. C.P.M. Reutelingsperger

Beoordelingscommissie:

Prof. dr. M.P. Van Dieijen-Visser (voorzitter)

Prof. dr. P.J.A. Borm (HHU Düsseldorf/ Magnamedics Diagnostics BV)

Dr. E. Lutgens

Prof. dr. J. Narula (University of California, Irvine)

Prof. dr. F.C.S. Ramaekers

Financial support by the Netherlands Heart Foundation for the publication of this thesis is gratefully acknowledged.

Additional financial support was kindly provided by AstraZeneca, Medtronic Bakken Research Center, MosaMedix Health Care, and Schering-Plough. 
$\begin{array}{ll}\text { One } \quad \text { General Introduction } & 7\end{array}$

$\begin{array}{ll}\text { Two Imaging of atherosclerosis in murine arteries } & 13\end{array}$

Three Nanoparticles for optical molecular imaging of atherosclerosis $\quad 33$

Four Optical and magnetic resonance imaging of cell death and 79 platelet activation using Annexin A5-functionalized quantum dots

Five Hot-spot labeling of apoptosis and collagen in atherosclerotic 105 plaques using bimodal quantum dots

Six Molecular MRI of early thrombus formation using a bimodal $\alpha_{2^{-}} \quad 131$ antiplasmin-based contrast agent

$\begin{array}{lll}\text { Seven } & \text { General discussion } & 151\end{array}$

$\begin{array}{lll}\text { Eight } & 163\end{array}$

$\begin{array}{ll}\text { Samenvatting } & 166\end{array}$

$\begin{array}{ll}\text { Dankwoord } & 171\end{array}$

$\begin{array}{ll}\text { Curriculum Vitae } & 174\end{array}$

$\begin{array}{ll}\text { List of publications } & 175\end{array}$

$\begin{array}{lll}\text { Nine } & \text { Full color figures } & 177\end{array}$ 


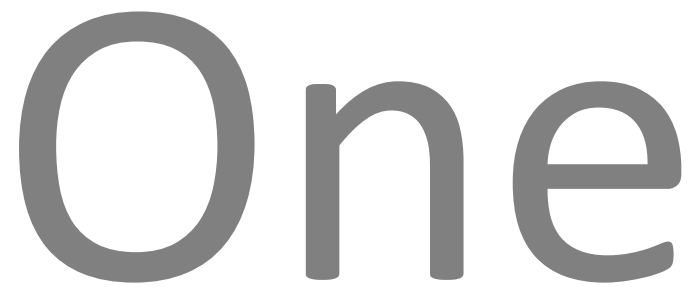

General introduction 


\section{Chapter 1}

Most humans develop atherosclerosis to some extent during aging. It can be accelerated by, e.g., genetic predisposition, a Western diet, or smoking. Atherosclerosis is a disease in which cholesterol esters are deposited inside the vascular wall at certain sites, called atherosclerotic plaques. This deposition may lead to complete or partial occlusion of the vessel or to rupture of the plaque, causing thrombosis. Plaques usually develop at sites of disturbed laminar flow, leading to low and oscillatory shear stress on the endothelial cells ${ }^{1}$. Examples of such sites are the bifurcation in the abdominal aorta, the bifurcation in the common carotid artery, and the many bifurcations in the coronary arteries. Low or oscillatory shear stress induces mechanotransductionmediated alterations in protein expression in endothelial cells ${ }^{1}$, which can lead to endothelial activation under the influence of risk factors ${ }^{1}$. Endothelial activation in turn may induce inflammation ${ }^{2,3}$. Activated endothelial cells express adhesion molecules that allow platelets and different subsets of leukocytes to adhere to the endothelium ${ }^{2}$ ${ }^{4,5}$. After adhesion, leukocytes enter the vascular wall ${ }^{2}$. Once inside the vascular wall, monocytes, one of the subsets of leukocytes, differentiate to macrophages and start to endocytose lipoproteins ${ }^{2}$. In time, plaques increase in size by taking up more lipids and leukocytes. Macrophages endocytose more lipids, forming foam cells, and this eventually results in development of a necrotic core. Fibrous caps that consist mainly of collagen and elastin develop and cover the plaque. When plaques become advanced extracellular matrix degrading enzymes degrade the fibrous cap, which may cause cap rupture. In this process the necrotic core of the plaque is exposed to the blood stream, causing thrombosis. This may lead to the occlusion of the artery at this site or at a site more downstream, causing ischemia in the tissue distal to the occlusion, e.g., a myocardial or cerebral infarction.

Prevention of such ischemic events would lead to a major decrease of morbidity and mortality in the industrialized countries, and is thus one of the major research areas in biomedical science. Currently, rupture-prone sites are detected after an ischemic event has taken place by performing angiography. This is a technique in which an X-ray contrast agent is injected intravenously in the patient, visualizing the arterial lumen. The occluded artery as well as the culprit lesion is identified as a narrowed artery. Patients are treated with thrombolytic therapy, angioplasty of stenotic plaques, or both. How- 
ever, the culprit lesion is not necessarily a stenotic plaque, as rupture-prone plaques may very well be (or perhaps even in most cases ${ }^{1,6}$ ) outward remodeled plaques, and thus not visible in angiography. Furthermore, it would be convenient if these ruptureprone plaques were identified prior to actual events. In other words, atherosclerotic patients-at-risk should be screened for rupture-prone plaques so that preventative action can be taken.

Molecular imaging is an emerging branch of biomedical science, in which contrast agents are developed that bind molecular targets specific for a certain pathology. This concept has major advantages. First, as these contrast agents bind with molecular specificity, aspecific localization of the contrast agents is reduced, and diagnosis is more accurate. Second, as pathologies induce molecular changes prior to clinical events this disease can be detected prior to these events, and preventative measures can be taken. Third, as the aspecific binding of these contrast agents is considerably reduced, a lower dose is needed, reducing possible side-effects for the patient.

Imaging can be performed using several techniques, such as fluorescence imaging, magnetic resonance imaging (MRI), computed tomography (CT), positron emission tomography (PET), or single photon emission computed tomography (SPECT). They all have their own advantages and disadvantages in research and in clinical use. Fluorescence imaging provides a high (sub-cellular) resolution ( $\mu \mathrm{m}$ range) and high sensitivity. Some anatomical information is provided as some tissue-types exhibit autofluorescence. However, fluorescence imaging is usually invasive due to the low penetration depth of light and is not often used as a clinical imaging technique. New optical, potentially clinical techniques are being developed, as discussed in chapter 2. CT offers a reasonable resolution ( $\mathrm{mm}$ range) and sensitivity and provides some anatomical information, but is an imaging technique using radiation, and iodinated contrast agents are necessary for visualization. Thus, CT is potentially harmful to patients. PET and SPECT offer high sensitivity but low resolution (several $\mathrm{mms}$ ) and no anatomical information. Furthermore, they are also imaging techniques using radiation and requiring radioactive contrast agents. MRI has a relatively high resolution (sub-mm range), and is a noninvasive technique providing anatomical information, but is not very sensitive to contrast agents. 


\section{Chapter 1}

In this thesis, three different nanoparticles were developed. Each nanoparticle targets a molecular epitope specific for either atherosclerosis or thrombosis, or both. All three nanoparticles were bimodal, i.e., visible with both fluorescence microscopy and MRI. In this way, advantages of both imaging techniques are combined. Two atherosclerosistargeting nanoparticles consisted of a fluorescent, streptavidin-coated quantum dot, which is approximately $10 \mathrm{~nm}$ in diameter. One nanoparticle targets dying cells, the other targets collagen present in atherosclerotic plaques. MRI contrast was provided by Gadolinium-DTPA wedges, each containing 8 Gadolinium-DTPA moieties for signal amplification. The two nanoparticles contained different ligands, each with their own molecular targets. The third nanoparticle is a peptide-based, thrombus-targeting nanoparticle, and is much smaller $(<<1 \mathrm{~nm})$. A sequence of 12 amino acids with known binding properties to fibrin was used, to which both an organic fluorescent dye (rhodamine) and Gadolinium-DTPA were covalently attached. All three nanoparticles were first tested in vitro on cells and/or blood clots as a proof-of-principle. Next, mouse models of atherosclerosis and thrombosis were used to establish the feasibility of the three bimodal nanoparticles to image either pathology in carotid arteries. Arteries were imaged both in vivo with MRI and ex vivo with two-photon laser scanning microscopy (TPLSM), in order to allow both non-invasive imaging with (relatively) high resolution and subcellular resolution for validation of the molecular localization of the nanoparticle.

Chapter 2 is an introductory chapter, discussing the structure of arterial walls. Next, atherosclerosis in mice is discussed, explaining the difference between atherosclerosis in mice and men. Then, biochemical processes of cell death and blood coagulation are discussed. Furthermore, the physical principles of two-photon laser scanning microscopy (TPLSM) are reviewed. Also, it is illustrated how plaques were imaged using TPLSM. In chapter 3, optical imaging techniques and novel optical en hybrid (bimodal) nanoparticles are reviewed, followed by the development of atherosclerosis in humans and appropriate molecular targets and corresponding targeting ligands. Furthermore, the clinical potential of these imaging techniques and nanoparticles is discussed. In chapter 4, the proof-of-principle of a bimodal quantum dot for the detection of apoptosis and activated platelets is provided in an in vitro and ex vivo carotid artery study. Chapter 5 describes an in vivo mouse study using the bimodal nanoparticle from chap- 
ter 4, functionalized with two different molecular targets, to visualize atherosclerosis in mice. Here, it is determined if this bimodal contrast agent is applicable to atherosclerotic mice, imaged in vivo with MRI and ex vivo with TPLSM. In chapter 6 a peptidebased bimodal nanoparticle is described, that is tested in an in vitro study providing the proof-of-principle, followed by an in vivo mouse study of fresh thrombi in carotid arteries using in vivo MRI and ex vivo TPLSM. Finally, chapter 7 is the general discussion of this thesis. 


\section{Chapter 1}

\section{References}

1 Y. S. Chatzizisis, A. U. Coskun, M. Jonas, E. R. Edelman, C. L. Feldman and P. H. Stone; "Role of endothelial shear stress in the natural history of coronary atherosclerosis and vascular remodeling: molecular, cellular, and vascular behavior", J Am Coll Cardiol 2007, 49(25) 2379-93.

R. R. Packard and P. Libby; "Inflammation in atherosclerosis: from vascular biology to biomarker discovery and risk prediction", Clin Chem 2008, 54(1) 24-38.

V. Braunersreuther, F. Mach and S. Steffens; "The specific role of chemokines in atherosclerosis", Thromb Haemost 2007, 97(5) 714-21.

C. Weber, A. Zernecke and P. Libby; "The multifaceted contributions of leukocyte subsets to atherosclerosis: lessons from mouse models", Nat Rev Immunol 2008, 8(10) 802-15.

S. Lindemann, B. Kramer, P. Seizer and M. Gawaz; "Platelets, inflammation and atherosclerosis", J Thromb Haemost 2007, 5 Suppl 1(203-11.

R. Virmani, A. P. Burke, A. Farb and F. D. Kolodgie; "Pathology of the vulnerable plaque", J Am Coll Cardiol 2006, 47(8 Suppl) C13-8. Egbrink and M. A. van Zandvoort; "Two-Photon Microscopy of Vital Murine Elastic and Muscular Arteries. Combined Structural and Functional Imaging with Subcellular Resolution", J Vasc Res 2006, 44(2) 87-98. 


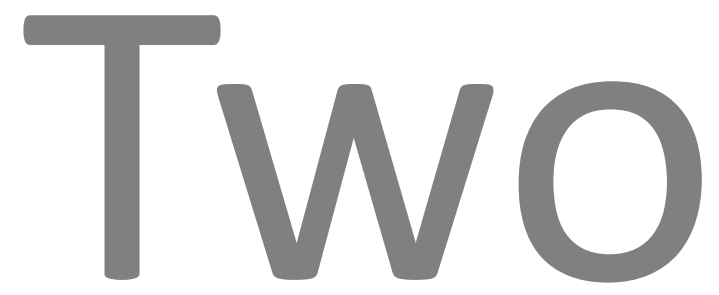

Imaging of atherosclerosis in murine arteries 


\section{Chapter 2}

In this chapter, several subjects will be discussed as an introduction to this thesis. These subjects include vascular wall structure, differences in atherosclerosis development between mice and humans, cell death in atherosclerotic plaques, the blood coagulation cascade, the physical principles of two-photon imaging, and plaque imaging with TPLSM.

\section{Arterial wall structure}

The mammalian arterial wall consists of three different layers; tunica intima, tunica media, and tunica adventitia (figure 1). Tunica intima is the most luminal layer, consisting of the endothelium, underlying connective tissue (the basal lamina), and the internal elastic lamina. The endothelium consists of a single layer of endothelial cells, and provides an antithrombotic surface, preventing blood from coagulating under normal circumstances. Furthermore, endothelial cells monitor and regulate many processes, such as vasoconstriction and vasodilatation by releasing regulatory factors, permeability to specific molecules, and, in case of inflammation, capturing and allowing trans- and paracellular migration of inflammatory cells ${ }^{1,2}$. The luminal side of endothelial cells is covered by a matrix of proteoglycans and glycoproteins called the glycocalyx. This matrix also regulates vascular permeability and may induce mechanotransduction ${ }^{3}$. The basal lamina connects the endothelial cells to the vascular wall; it is a structure of extracellullar matrix components such as collagen. Collagen is a fibrillar protein that provides the strength of the vascular wall. The internal elastic lamina separates the tunica intima from the tunica media. It consists of elastin, an extracellullar matrix protein that provides the elasticity of the vascular wall.

The second layer is the tunica media, which consists of several layers of smooth muscle cells. These smooth muscle cells are enclosed by a basal lamina and connective tissue (collagen types I, III, and IV, and elastin). Furthermore, in between these layers of cells elastic laminae are present ${ }^{2}$. The number of elastic laminae depends on the size and type of the artery: large arteries, such as aorta and carotid arteries, are more elastic and contain more elastic laminae. This is because these arteries have to deal with large pulsations in arterial pressure. Muscular arteries are present further downstream, and 


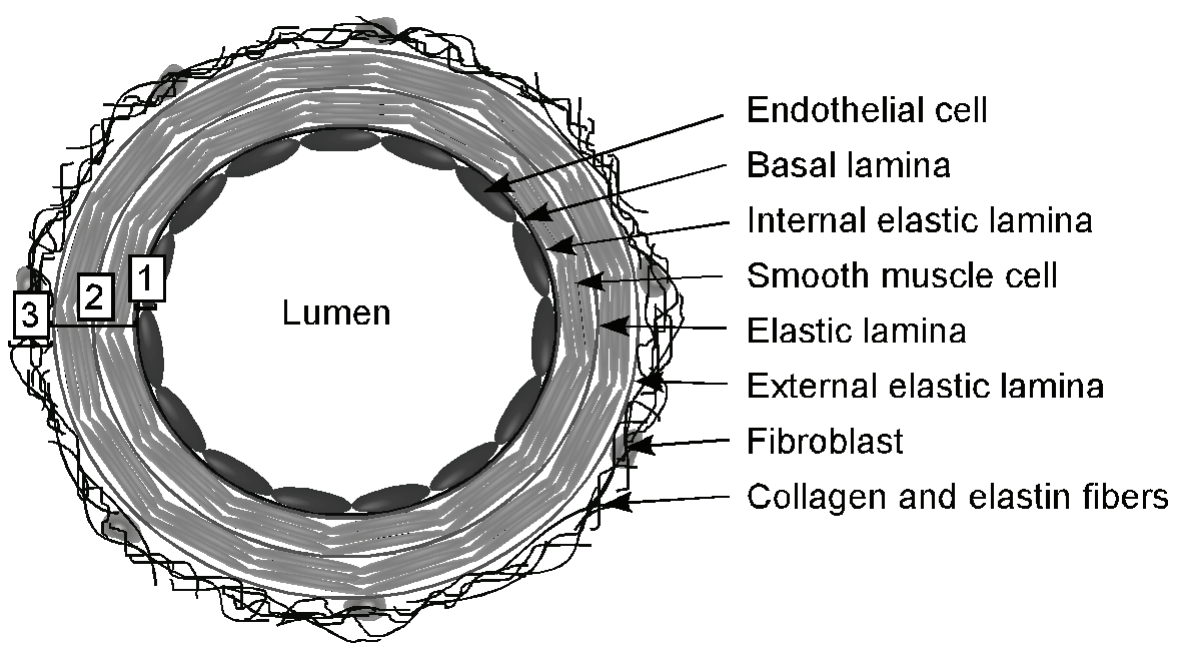

Figure 1. Schematic representation of transverse view of arterial wall of a murine carotid artery. ' 1 ' indicates tunica intima, ' 2 ' tunica media, and ' 3 ' tunica adventitia. Color figure on page 178.

contain fewer elastic laminae. Unlike the name suggests, muscular arteries actually have a lower smooth muscle cell density than elastic arteries ${ }^{4}$.

The external elastic lamina separates the tunica media from the tunica adventitia, the outer most layer of the vascular wall. The adventitial layer consists of collagen type I, elastin fibers, and fibrotic cells ${ }^{2}$, and embeds the artery in the surrounding tissue.

With increasing lumen diameter, vascular wall thickness generally also increases. When arteries are larger than, roughly, $0.5 \mathrm{~mm}$ in lumen diameter, transmural blood supply to the outer vascular wall layers is not sufficient, and vasa vasora are necessary ${ }^{5}$. These are small vessels supplying the tunica adventitia with nutrients and oxygen. Generally, murine arteries do not have vasa vasorum under normal circumstances as most arteries have a luminal diameter smaller than $0.5 \mathrm{~mm}$. However, when there is a large plaque present, it may be supplied by a vasa vasorum. For example, in ApoE/LDL double knockout mice, vasa vasora were observed in adventitia in case of a large plaque in combination with a lumen diameter larger than $0.4 \mathrm{~mm}^{6}$. 


\section{Chapter 2}

Besides endothelial cells, smooth muscle cells, and fibroblasts, other types of cells may also be present within the vascular wall, such as dendritic cells, T-cells, and endothelial progenitor cells, but it is outside the scope of this thesis to discuss these.

This study investigates murine carotid arteries. In healthy conditions, they consist of an intimal layer, a media consisting of several layers of smooth muscle cells and three elastic laminae, and an adventitium (figure 1).

\section{Atherosclerosis in mice}

Mouse models of atherosclerosis are widely used to understand biology of atherogenesis. Normal mice do not develop atherosclerosis, since mice hardly have circulating LDL (the 'bad' cholesterol) as humans do, but mainly have circulating HDL (the 'good' cholesterol) ${ }^{7}$. Therefore, genetic modifications have been introduced to disturb their lipid metabolism. The most common mouse model for atherosclerosis research is the Apolipoprotein $\mathrm{E}$ knockout mouse (ApoE -/- mouse) ${ }^{7}$. Apolipoprotein $\mathrm{E}$ is a ligand involved in lipoprotein clearance, and ApoE -/- mice have a delayed lipoprotein clearance ${ }^{7}$. Especially when fed a high-fat diet, ApoE -/- mice have increased levels of circulating chylomicron and VLDL remnants, leading, over the course of approximately 40 weeks (a mouse's lifetime), to the development of fibrous lesions that closely resemble human plaques ${ }^{7,8}$. These plaques are located mainly at sites of oscillatory shear stress, such as at branching arteries (e.g. at the branch points of the carotids at the aorta, and at the bifurcation of the carotids into the internal and external carotids), but also in the curvature of the aortic arch ${ }^{7,8}$. When ApoE -/-mice are fed a high-fat diet, these plaques start to develop after 6 weeks of age with monocyte adhesion to the endothelium. This is presumably preceded by the adhesion of platelets to activated endothelium ${ }^{9}$. After about 8 weeks the first foam cells appear in lesions. At 10 weeks, intermediate lesions containing more foam cells and smooth muscle cells start to develop, followed by progression to advanced lesions containing necrotic cores at 15 weeks of age. Lesions progress further by increasing in size and advancing occlusion of the artery ${ }^{8}$. This shows great resemblance with human plaque formation (see chapter 3 ), except on a time scale of weeks instead of years. 
To increase the rate of plaque development, Von der Thüsen et al. ${ }^{10}$ developed a modified mouse model of atherosclerosis. They used the ApoE -/- mouse on a high-fat diet in combination with a silicon collar placed around the common carotid artery, proximal to the bifurcation. This collar is mildly constrictive ( $30 \%$ stenosis) ${ }^{10}$, and induces plaque formation proximal to the collar, presumably due to disturbance of laminar flow proximal to the collar ${ }^{10}$. In this mouse model plaque progression is accelerated; already 6 weeks after collar placement advanced lesions are present. This model was used in chapter 5 of this thesis. These plaques resemble those in spontaneous atherosclerosis in ApoE -/- mice; heterogeneous in composition, an acellular necrotic core, a welldefined fibrous cap, significant extracellular matrix composition, intra- and extracellular lipids, lipid-rich foam cells, and smooth muscle cells in the intima after 6 weeks of collar-placement ${ }^{10}$.

Murine plaques are usually of a stable, fibrous phenotype that ruptures only sporadically ${ }^{11}$. In humans, plaque rupture is the most common cause for acute events such as myocardial infarction and stroke. Spontaneous rupture in ApoE -/- mice does occur, however at a far too low frequency to make it a reliable and reproducible model for studying plaque rupture ${ }^{12}$. Furthermore, murine plaque morphology differs from human plaque morphology. The latter has a well-defined fibrous cap preventing contact between blood and the underlying necrotic core. The fibrous cap can rupture which results in exposure of the necrotic core to the blood and a subsequent thrombotic response. Murine plaques do not have a well-defined fibrous cap ${ }^{11}$, although Von der Thusen et al. did describe plaques in mice that had a collar for 6 weeks as having a welldefined fibrous cap ${ }^{10}$. 'Rupture' described in these plaques may refer to either the disintegration of endothelium or deep erosion of the necrotic core ${ }^{11,12}$, both of which may occasionally lead to thrombosis. Several mouse models have been developed in an attempt to resemble human plaque rupture ${ }^{13-16}$. Schwartz et al. state in their review discussing plaque ruptures in mice and humans ${ }^{11}$ that an animal model of plaque rupture should presuppose the existence of a fibrous cap overlying a necrotic core, as this is required in human plaque rupture. This has so far not been achieved in mice.

In chapter 5 , the collar model was used in order to determine effectiveness of new bimodal contrast agents, because: i) the collar model has a rapid rate of plaque progres- 


\section{Chapter 2}

sion, and ii) the collar determines the location of plaque formation, facilitating visualization of the plaque by MRI. This model also has disadvantages. First, due to the invasive nature of collar placement, the survival rate of these mice is lower. Furthermore, due to collar placement, fibrous tissue develops around the collar, hindering optical imaging.

\section{Cell death in atherosclerosis}

Several types of cell death occur in living organisms. The two best defined types of cell death are necrosis and apoptosis. Necrosis is a process of cell death in which the cell increases in size and eventually bursts, inducing inflammation ${ }^{17}$. Apoptosis is a form of programmed cell death, in which the cell shrinks, divides into multiple apoptotic bodies, and is cleared by macrophages without leading to inflammation ${ }^{17}$. Apoptosis is a process necessary for homeostasis in any multicellular organism, and also plays an essential role in several pathologies. In tumor development, increased cell division as well as decreased apoptosis leads to excessive tissue growth, while after myocardial infarction apoptosis increases. Other, less well-defined types of cell death also exist ${ }^{18}$, but are not discussed here.

A common phenomenon in many types of cell death is the exposure of phosphatidylserine (PS) ${ }^{19}$ on the cellular membrane, which, in viable cells, is only exposed on the cytoplasmic membrane leaflet. In atherosclerosic plaques PS-exposing cells are present. Smooth muscle cells ${ }^{20}$, macrophages ${ }^{21}$, and presumably also endothelial cells ${ }^{22}$ may undergo apoptosis in plaques, contributing to plaque vulnerability. Furthermore, activated macrophages ${ }^{23}$ may expose PS, as well as activated platelets ${ }^{24}$. The presence of apoptotic cells, macrophages, and platelets in plaques are all a measure of plaque vulnerability. In other words, PS imaging is a potentially appropriate tool for vulnerable plaque detection $^{25}$.

Annexin A5 (AnxA5) is a ligand well-known for its high-affinity binding to PS ${ }^{26}$. AnxA5 is a member of the Annexin-family, the members of which share structural and functional features ${ }^{27}$. The primary structure of an annexin is composed of a divergent $\mathrm{N}$-terminal tail and a conserved C-terminal core that consists of 4 or 8 homologous domains. The conserved domains harbor the $\mathrm{Ca}^{2+}$ and phospholipid binding sites. The tertiary structure of an annexin has a bent shape with a convex and a concave side containing the 
phospholipid binding sites and the $\mathrm{N}$-terminal tail, respectively ${ }^{27}$. When bound to a phospholipid surface the concave side with the $\mathrm{N}$-terminal tail faces away from the phospholipid surface and stays in contact with the medium surrounding the membrane. The annexins differ in their affinities for binding various phospholipid species. To date AnxA5 is the annexin with the highest reported affinity for PS binding. AnxA5 can be coupled to several contrast agents to allow imaging with different techniques, such as radiographic or fluorescent imaging. AnxA5 has been used in multiple pre-clinical and clinical studies. Examples of pathologies imaged with AnxA5 are endocarditis ${ }^{28}$, thrombosis ${ }^{29}$, atherosclerosis ${ }^{30,31}$, abdominal aortic aneurysm ${ }^{32}$, ischemic tissue after myocardial infarction ${ }^{33-35}$, heart failure ${ }^{36}$, liver apoptosis ${ }^{37}$, and radiation-induced apoptosis in anti-tumor treatment ${ }^{38,39}$.

\section{Thrombosis}

Thrombosis is the major event leading to infarctions after plaque rupture. Thrombi bound to the vascular wall (mural thrombi) are thus also a suitable target for detection of vulnerable plaques. The pathway leading to thrombus formation is the coagulation cascade. The initiation phase of the cascade encompasses the complexation of cellbound tissue factor (TF) with factor VII. In vascular surroundings, TF may be expressed on smooth muscle cells, fibroblasts, and in case of inflammation, also on endothelial cells and monocytes ${ }^{40}$. Also necessary for initiation is binding of platelets to collagen or other extracellular matrix components after vascular wall damage, leading to platelet activation ${ }^{41}$. Platelet activation leads to PS exposition, and coagulation factors are activated. In the amplification phase, small amounts of thrombin are exposed by TF-bearing cells, resulting in an amplification of the activation of coagulation factors by activated platelets ${ }^{41}$. Finally, in the propagation phase, activation of more coagulation factors occurs, resulting in the conversion of large amounts of prothrombin into thrombin ${ }^{41}$. Thrombin converses circulating fibrinogen into fibrin, which is followed by cross-linking of fibrin fibers under the influence of activated factor XIII, forming a stabilized fibrin clot. $\alpha_{2}$-antiplasmin is also cross-linked to fibrin under the influence of activated factor XIII ${ }^{42}$, making the clot more resistible to fibrinolysis. Eventually, over the course of 


\section{Chapter 2}

hours, a stabilized clot develops consisting of activated platelets, erythrocytes, and leucocytes, in a matrix of cross-linked fibrin.

This coagulation cascade gives rise to many molecular targets of thrombosis. Fibrin is perhaps the easiest target within a clot, and has been used in several studies ${ }^{43-48}$. In chapter 6 of this thesis, an $\alpha_{2}$-antiplasmin-based contrast agent is used to target fibrin. Also activated platelets are a potential target ${ }^{49,50}$, for example by targeting PS ${ }^{32}$.

\section{Two-photon laser scanning microscopy}

Two-photon laser scanning microscopy (TPLSM) is a type of fluorescence imaging in which two photons instead of a single photon are used to excite a fluorescent molecule (figure 2). These photons each have about half the energy and a wavelength that is about double that of the photons used in single photon imaging, as the wavelength is inversely related to its energy:

$$
E=\frac{h c}{\lambda}
$$

with $\mathrm{E}$ the energy, $\mathrm{h}$ Planck's constant, $\mathrm{c}$ the speed of light and $\lambda$ the wavelength.

These two photons have to arrive at the molecule within $10^{-18} \mathrm{~s}$ in order to induce excitation ${ }^{51}$. This means that a high density of photons is needed at the focal point of the laser beam in order to achieve a measurable amount of excitation, and thus also emission. This density needs to be approximately $10^{31}$ photons $/ \mathrm{cm}^{2} 52,10^{6}$ times higher than for single-photon excitation ${ }^{51}$. For this reason, photons are concentrated both in spatial and temporal dimensions. Temporal concentration of photons is achieved by a pulsed laser beam instead of a continuous one. Pulsation of the laser beam leads to highintensity pulses, in turn leading to sufficient excitation, while the average laser power remains low ${ }^{51}$. A pulse duration of about 100 femtoseconds $\left(1 \mathrm{fs}=10^{-15} \mathrm{~s}\right)$ and a repetition rate of $80 \mathrm{MHz}^{53}$ (1 pulse per 12.5 nanoseconds; $1 \mathrm{~ns}=10^{-9} \mathrm{~s}$ ) are required for a measurable amount of two-photon excitation (figure 3). Spatial concentration is achieved by tight focusing of the laser beam. The laser has to have a very high intensity; an incident laser power in the order of $50 \mathrm{~mW}$ is required ${ }^{53}$. This is large compared to the incident laser light of confocal microscopy: $\approx 3 \mathrm{~mW}^{53}$. 


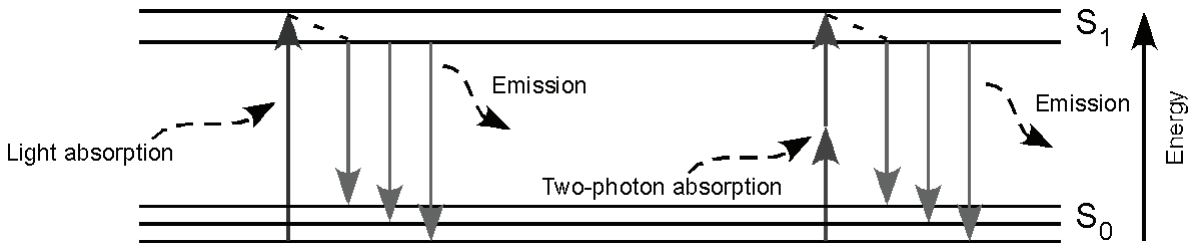

Figure 2. Simplified Jablonski diagram showing single (blue) and two (red) -photon excitation. Color figure on page 178.

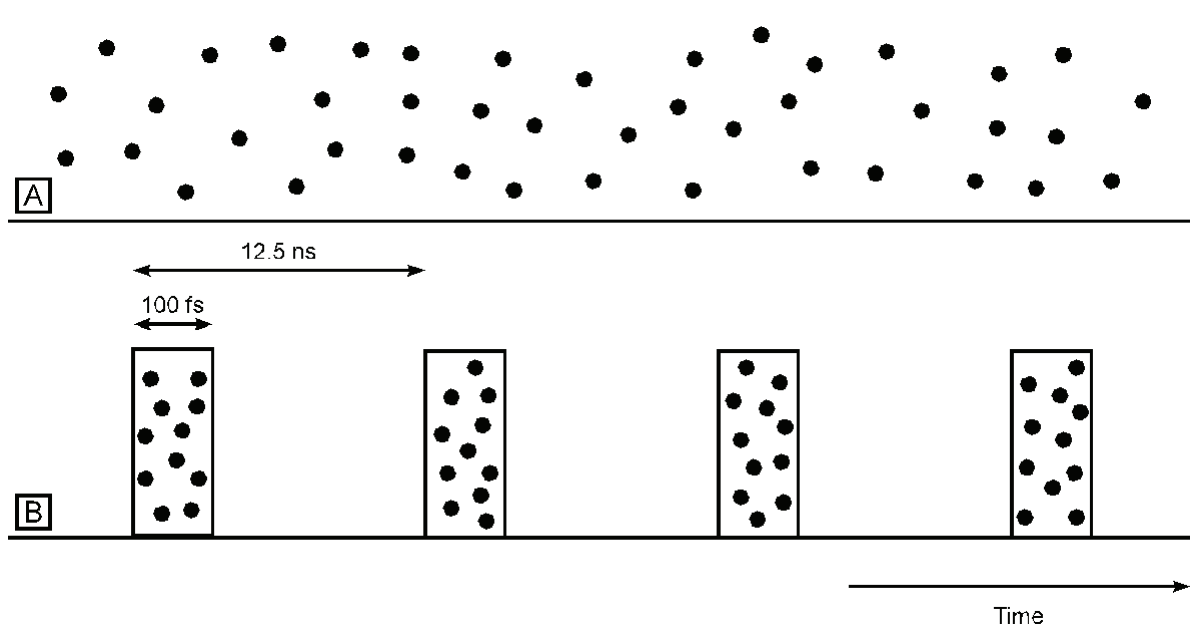

Figure 3. Schematic representation of photons in a continuous (A) and pulsed (B) laser beam.

As the absorption of photons by fluorescent molecules depends on the square of the excitation intensity, and because the excitation intensity decreases with the square of the distance to the focal point, the two-photon excitation probability falls with the fourth power with distance to the focal point ${ }^{52}$. This means that, effectively, only in the focal point a measurable amount of two-photon excitation and consequent fluorescence emission occurs (figure 4). Thus, with TPLSM, no out-of-focus excitation occurs. This results in intrinsic optical sectioning making 3D reconstruction possible without the use of pinholes, as required in confocal microscopy. Also, no photodamage or photobleaching occurs outside of the focal point, since no excitation occurs outside focus. This is an important advantage over single-photon excitation, such as confocal imaging. Another advantage is that the penetration depth in tissue is larger. This is due to the 

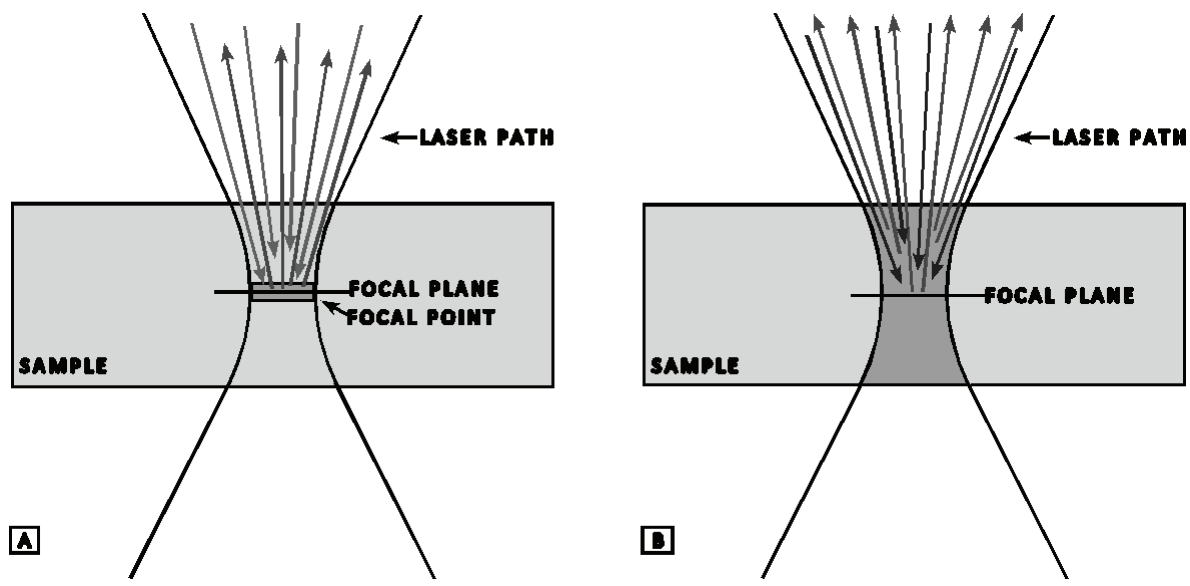

Figure 4. Schematic representation of excitation and fluorescence emission in TPLSM (A) and confocal microscopy (B). In TPLSM, near infrared laser light (indicated by red arrows) is used to excite molecules in the focal point. As excitation only occurs in the focal point, all of the green emitted light (indicated by green arrows) originates from the focal point, and can be used for reconstruction of the image. In confocal microscopy, blue/green laser light is used for excitation, as indicated by blue arrows. Molecules are excited along the laser path, not necessarily only in the focal plane. Color figure on page 179.

absence of out-of-focus absorption and due to the longer excitation wavelength. Excitation is in the near infrared range $(700-1100 \mathrm{~nm})$, reducing scattering of light in tissue.

Two-photon absorption and emission were first predicted by Maria Göppert-Mayer already in 1931. Two-photon excitation was not possible until the discovery of the laser in $1960{ }^{54}$, and the development of a femtosecond-pulsed laser in $1971^{54}$. Denk et al. were the first to apply two-photon excitation fluorescence microscopy to image biological samples in $1990^{53}$. They described the combination of a femtosecond pulsed laser with a laser scanning microscope, which allowed two-photon laser scanning microscopy (TPLSM). Since then, many papers have been published in which TPLSM is used to image biological tissues with high resolution and at larger penetration depth. Examples of application areas are: neurology ${ }^{55-59}$, immunology ${ }^{60-62}$, nephrology ${ }^{63,64}$, and vascular biology $3,4,65$.

\section{Plaque imaging with TPLSM}

Atherosclerotic plaques are difficult to visualize with fluorescence imaging due to the lack of penetration depth in fat. Three different methods were employed to visualize 
the plaque as much as possible. The first method was described by Megens et al. ${ }^{4}$, using flow chambers in which arteries are mounted on glass micropipettes. In this flow chamber, arteries can be visualized while under static transmural pressure, usually 80 $\mathrm{mmHg}$. Visualization is performed from the adventitial side to the luminal side of the vascular wall (figures $5 \mathrm{~A}$ and $6 \mathrm{~A}$ ). In this method, shoulders of plaques can be imaged well; however, the luminal side and the center of the plaque is usually undetectable when plaques are too thick, i.e. $>100 \mu \mathrm{m}^{66}$. Therefore, in the second method, carotids are carefully dissected in longitudinal direction (figures $5 B$ and $6 B$ ), and placed flat on silicon gel. This way, the plaque can be imaged from the luminal side. However when plaques are too thick, again not the entire plaque can be visualized. In the third method, the artery is dissected in transverse direction and placed in agarose gel (figures $5 \mathrm{C}$ and $6 \mathrm{C}$ ). Now, the plaque can be visualized from the adventitial to the luminal side, but only few tens of micrometers in longitudinal direction. In other words, all three methods have advantages as well as disadvantages.

Atherosclerotic plaques have previously been imaged with TPLSM. Megens et al. have performed a study in which CNA-OG488 was investigated for its ability to label fibrous caps in plaques ${ }^{67}$. Here, mounted carotid arteries, including the bifurcation, of old ApoE -/- mice were examined for the presence of CNA-OG488 in combination with a nucleic acid stain. In a follow-up study, the interaction of inflammatory cells with collagen in plaques was investigated ${ }^{66}$. Yu et al. performed an in vivo study in which they investigated murine carotid arteries for the presence of lipids using Nile Red ${ }^{68}$. Another study used TPLSM in combination with second harmonic generation (SHG) to investigate human aortic samples for their intrinsic collagen and elastin luminiscence ${ }^{69}$. Yet another study combined TPLSM, SHG, and coherent anti-Stokes Raman scattering (CARS) for visualization of several plaque components (collagen, elastin, and lipids) in ex vivo pig iliac arteries, also without the addition of any fluorescent label ${ }^{70}$. 

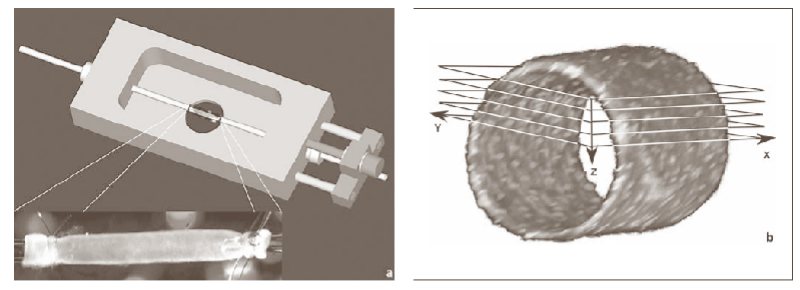

因
Figure 5. Schematic representation of artery visualization. A) Left: Artery in flow chamber. Right: Optical sections can be made from adventitial side to luminal side. From Megens et al. ${ }^{4}$. B) Artery is dissected in longitudinal direction and imaged from the luminal side. C) Artery is dissected in transverse direction and viewed as a transverse slice.

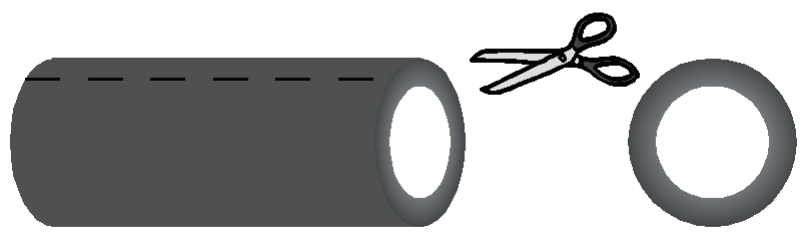

因

무
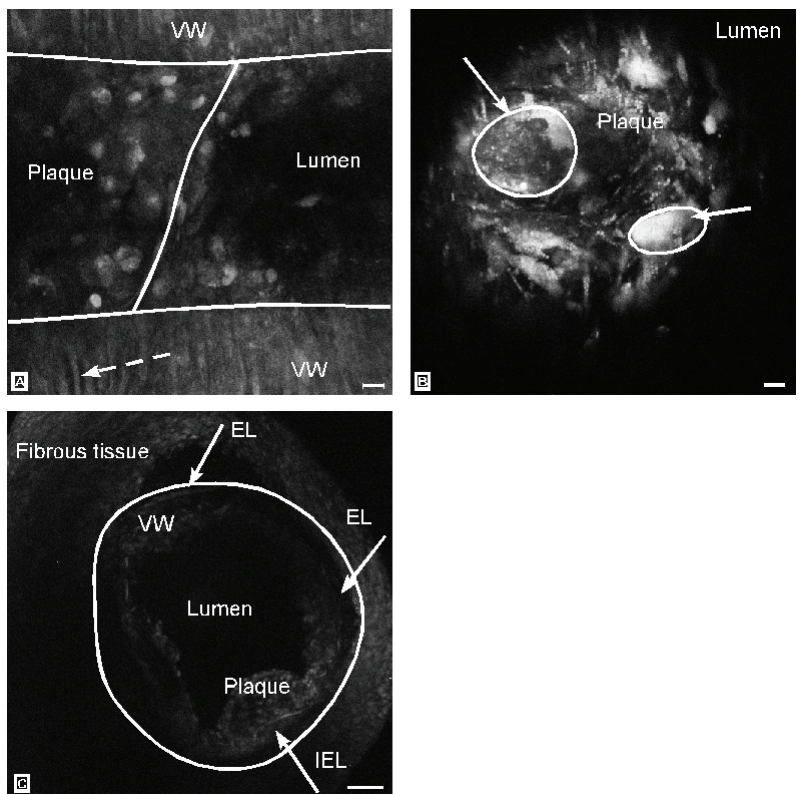

Figure 6. TPLSM images of plaque-containing carotid arteries with three different methods. A) Artery mounted in flow chamber. In the vascular wall, smooth muscle cells are visible; an example is indicated by dashed arrow. Also inside the plaque cells are visible. Endothelial cells are not visible in this image. B) Artery longitudinally dissected and imaged while in agarose gel, with the luminal side on top. Here, only the plaque is visible. Inside the plaque, foam cells containing multiple nuclei (indicated by circles) are visible. C) Artery transversally dissected. Circle indicates border between vascular wall and surrounding tissue. IEL indicates border between vascular wall and plaque (endothelium is not clearly visible in this image). The three elastic laminae are indicated by arrows. Blue: nuclei; red in B: lipids. VW: vascular wall, EL: elastic lamina, IEL: internal elastic lamina. In $A$ and $B, b a r=10 \mu \mathrm{m}$; in $C$, bar $=50 \mu \mathrm{m}$. Color figure on page 179 . 


\section{References}

1 K. Ley, C. Laudanna, M. I. Cybulsky and S. Nourshargh; "Getting to the site of inflammation: the leukocyte adhesion cascade updated", Nat Rev Immunol 2007, 7(9) 678-89.

L. Junqueira and J. Carneiro, "Basic Histology", 11th edition, 2005, The McGraw-Hill Companies, Inc.

S. Reitsma, D. W. Slaaf, H. Vink, M. A. van Zandvoort and M. G. Oude Egbrink; "The endothelial glycocalyx: composition, functions, and visualization", Pflugers Arch 2007,

R. T. Megens, S. Reitsma, P. H. Schiffers, R. H. Hilgers, J. G. De Mey, D. W. Slaaf, M. G. Oude Egbrink and M. A. van Zandvoort; "Two-Photon Microscopy of Vital Murine Elastic and Muscular Arteries. Combined Structural and Functional Imaging with Subcellular Resolution", J Vasc Res 2006, 44(2) 87-98.

E. L. Ritman and A. Lerman; "The dynamic vasa vasorum", Cardiovasc Res 2007, 75(4) 649658.

A. C. Langheinrich, A. Michniewicz, R. M. Bohle and E. L. Ritman; "Vasa vasorum neovascularization and lesion distribution among different vascular beds in ApoE-/-/LDL-/- double knockout mice", Atherosclerosis 2007, 191(1) 73-81.

J. L. Breslow; "Mouse models of atherosclerosis", Science 1996, 272(5262) 685-8.

Y. Nakashima, A. S. Plump, E. W. Raines, J. L. Breslow and R. Ross; "ApoE-deficient mice develop lesions of all phases of atherosclerosis throughout the arterial tree", Arterioscler Thromb 1994, 14(1) 133-40.

S. Massberg, K. Brand, S. Gruner, S. Page, E. Muller, I. Muller, W. Bergmeier, T. Richter, M. Lorenz, I. Konrad, B. Nieswandt and M. Gawaz; "A critical role of platelet adhesion in the initiation of atherosclerotic lesion formation", J Exp Med 2002, 196(7) 887-96.

J. H. von der Thusen, T. J. C. van Berkel and E. A. L. Biessen; "Induction of Rapid Atherogenesis by Perivascular Carotid Collar Placement in Apolipoprotein E-Deficient and Low-Density Lipoprotein Receptor-Deficient Mice", Circulation 2001, 103(8) 1164-1170.

S. M. Schwartz, Z. S. Galis, M. E. Rosenfeld and E. Falk; "Plaque rupture in humans and mice", Arterioscler Thromb Vasc Biol 2007, 27(4) 705-13.

F. Calara, M. Silvestre, F. Casanada, N. Yuan, C. Napoli and W. Palinski; "Spontaneous plaque rupture and secondary thrombosis in apolipoprotein E-deficient and LDL receptor-deficient mice", J Pathol 2001, 195(2) 257-63. 


\section{Chapter 2}

J. H. von der Thusen, B. J. van Vlijmen, R. C. Hoeben, M. M. Kockx, L. M. Havekes, T. J. van Berkel and E. A. Biessen; "Induction of atherosclerotic plaque rupture in apolipoprotein E-/mice after adenovirus-mediated transfer of p53", Circulation 2002, 105(17) 2064-70.

T. Sasaki, M. Kuzuya, K. Nakamura, X. W. Cheng, T. Shibata, K. Sato and A. Iguchi; "A simple method of plaque rupture induction in apolipoprotein E-deficient mice", Arterioscler Thromb Vasc Biol 2006, 26(6) 1304-9.

J. Johnson, K. Carson, H. Williams, S. Karanam, A. Newby, G. Angelini, S. George and C. Jackson; "Plaque rupture after short periods of fat feeding in the apolipoprotein E-knockout mouse: model characterization and effects of pravastatin treatment", Circulation 2005, 111(11) 1422-30.

M. J. Kuijpers, K. Gilio, S. Reitsma, R. Nergiz-Unal, L. Prinzen, S. Heeneman, E. Lutgens, V. A. N. Z. MA, B. Nieswandt, M. G. Oude Egbrink and J. W. Heemskerk; "Complementary roles of platelets and coagulation in thrombus formation on plaques acutely ruptured by targeted ultrasound treatment: a novel intravital model", J Thromb Haemost 2009, 7(1) 152-61.

G. Kroemer, L. Galluzzi, P. Vandenabeele, J. Abrams, E. S. Alnemri, E. H. Baehrecke, M. V. Blagosklonny, W. S. El-Deiry, P. Golstein, D. R. Green, M. Hengartner, R. A. Knight, S. Kumar, S. A. Lipton, W. Malorni, G. Nunez, M. E. Peter, J. Tschopp, J. Yuan, M. Piacentini, B. Zhivotovsky and G. Melino; "Classification of cell death: recommendations of the Nomenclature Committee on Cell Death 2009", Cell Death Differ 2009, 16(1) 3-11.

G. Kroemer, W. S. El-Deiry, P. Golstein, M. E. Peter, D. Vaux, P. Vandenabeele, B. Zhivotovsky, M. V. Blagosklonny, W. Malorni, R. A. Knight, M. Piacentini, S. Nagata and G. Melino; "Classification of cell death: recommendations of the Nomenclature Committee on Cell Death", Cell Death Differ 2005, 12 Suppl 2(1463-7.

M. F. Corsten, L. Hofstra, J. Narula and C. P. Reutelingsperger; "Counting heads in the war against cancer: defining the role of annexin A5 imaging in cancer treatment and surveillance", Cancer Res 2006, 66(3) 1255-60.

M. C. Clarke, N. Figg, J. J. Maguire, A. P. Davenport, M. Goddard, T. D. Littlewood and M. R. Bennett; "Apoptosis of vascular smooth muscle cells induces features of plaque vulnerability in atherosclerosis", Nat Med 2006, 12(9) 1075-80.

T. Seimon and I. Tabas; "Mechanisms and consequences of macrophage apoptosis in atherosclerosis", J Lipid Res 2008,

M. Clarke, M. Bennett and T. Littlewood; "Cell death in the cardiovascular system", Heart 2007, 93(6) 659-664. 
E. M. Laufer, C. P. Reutelingsperger, J. Narula and L. Hofstra; "Annexin A5: an imaging biomarker of cardiovascular risk", Basic Res Cardiol 2008, 103(2) 95-104.

J. F. Tait and D. Gibson; "Phospholipid binding of annexin V: effects of calcium and membrane phosphatidylserine content", Arch Biochem Biophys 1992, 298(1) 187-91.

M. F. Corsten, C. P. Reutelingsperger and L. Hofstra; "Imaging apoptosis for detecting plaque instability: rendering death a brighter facade", Curr Opin Biotechnol 2007, 18(1) 83-9. Heidendal and C. P. Reutelingsperger; "Past, present, and future of annexin a5: from protein discovery to clinical applications", J Nucl Med 2005, 46(12) 2035-50. 71.

F. Rouzet, M. Dominguez Hernandez, F. Hervatin, L. Sarda-Mantel, A. Lefort, X. Duval, L. Louedec, B. Fantin, D. Le Guludec and J. B. Michel; "Technetium 99m-labeled annexin V scintigraphy of platelet activation in vegetations of experimental endocarditis", Circulation 2008, $117(6) 781-9$.

T. Hayashi, H. Mogami, Y. Murakami, T. Nakamura, N. Kanayama, H. Konno and T. Urano; "Real-time analysis of platelet aggregation and procoagulant activity during thrombus formation in vivo", Pflugers Arch 2008,

S. Isobe, S. Tsimikas, J. Zhou, S. Fujimoto, M. Sarai, M. J. Branks, A. Fujimoto, L. Hofstra, C. P. Reutelingsperger, T. Murohara, R. Virmani, F. D. Kolodgie, N. Narula, A. Petrov and J. Narula; "Noninvasive imaging of atherosclerotic lesions in apolipoprotein E-deficient and low-densitylipoprotein receptor-deficient mice with annexin A5", J Nucl Med 2006, 47(9) 1497-505. and J. Narula; "Noninvasive detection of plaque instability with use of radiolabeled annexin A5 in patients with carotid-artery atherosclerosis", N Engl J Med 2004, 350(14) 1472-3. Touat, P. Merlet, D. Le Guludec and J. B. Michel; "99mTc-annexin-V functional imaging of luminal thrombus activity in abdominal aortic aneurysms", Arterioscler Thromb Vasc Biol 2006, 26(9) 2153-9.

D. E. Sosnovik, E. A. Schellenberger, M. Nahrendorf, M. S. Novikov, T. Matsui, G. Dai, F. Reynolds, L. Grazette, A. Rosenzweig, R. Weissleder and L. Josephson; "Magnetic resonance imaging of cardiomyocyte apoptosis with a novel magneto-optical nanoparticle", Magn Reson Med 2005, 54(3) 718-24. 


\section{Chapter 2}

34

L. Hofstra, I. H. Liem, E. A. Dumont, H. H. Boersma, W. L. van Heerde, P. A. Doevendans, E. De Muinck, H. J. Wellens, G. J. Kemerink, C. P. Reutelingsperger and G. A. Heidendal; "Visualisation of cell death in vivo in patients with acute myocardial infarction", Lancet 2000, 356(9225) 209-12.

5 L. Sarda-Mantel, J. B. Michel, F. Rouzet, G. Martet, L. Louedec, J. L. Vanderheyden, F. Hervatin, O. Raguin, J. M. Vrigneaud, B. A. Khaw and D. Le Guludec; "(99m)Tc-annexin V and (111)In-antimyosin antibody uptake in experimental myocardial infarction in rats", Eur J Nucl Med Mol Imaging 2006, 33(3) 239-45.

B. L. Kietselaer, C. P. Reutelingsperger, H. H. Boersma, G. A. Heidendal, I. H. Liem, H. J. Crijns, J. Narula and L. Hofstra; "Noninvasive Detection of Programmed Cell Loss with 99mTc-Labeled Annexin A5 in Heart Failure", J Nucl Med 2007, 48(4) 562-567.

H. G. Keen, B. A. Dekker, L. Disley, D. Hastings, S. Lyons, A. J. Reader, P. Ottewell, A. Watson and J. Zweit; "Imaging apoptosis in vivo using 124I-annexin V and PET", Nucl Med Biol 2005, 32(4) 395-402.

H. Watanabe, Y. Murata, M. Miura, M. Hasegawa, T. Kawamoto and H. Shibuya; "In-vivo visualization of radiation-induced apoptosis using (125)I-annexin V", Nucl Med Commun 2006, 27(1) 81-9.

9

R. L. Haas, D. de Jong, R. A. Valdes Olmos, C. A. Hoefnagel, I. van den Heuvel, S. F. Zerp, H. Bartelink and M. Verheij; "In vivo imaging of radiation-induced apoptosis in follicular lymphoma patients", Int J Radiat Oncol Biol Phys 2004, 59(3) 782-7.

A. K. Vine; "Recent advances in haemostasis and thrombosis", Retina 2009, 29(1) 1-7.

D. M. Monroe and M. Hoffman; "What does it take to make the perfect clot?" Arterioscler Thromb Vasc Biol 2006, 26(1) 41-8.

L. Muszbek, V. C. Yee and Z. Hevessy; "Blood Coagulation Factor XIII: Structure and Function", Thrombosis Research 1999, 94(5) 271-305.

M. R. Aruva, J. Daviau, S. S. Sharma and M. L. Thakur; "Imaging thromboembolism with fibrinavid 99mTc-peptide: evaluation in swine", J Nucl Med 2006, 47(1) 155-62.

E. Spuentrup, A. Buecker, M. Katoh, A. J. Wiethoff, E. C. Parsons, Jr., R. M. Botnar, R. M. Weisskoff, P. B. Graham, W. J. Manning and R. W. Gunther; "Molecular magnetic resonance imaging of coronary thrombosis and pulmonary emboli with a novel fibrin-targeted contrast agent", Circulation 2005, 111(11) 1377-82.

E. Spuentrup, B. Fausten, S. Kinzel, A. J. Wiethoff, R. M. Botnar, P. B. Graham, S. Haller, M. Katoh, E. C. Parsons, Jr., W. J. Manning, T. Busch, R. W. Gunther and A. Buecker; "Molecular 
Magnetic Resonance Imaging of Atrial Clots in a Swine Model", Circulation 2005, 112(3) 396399.

E. Spuentrup, M. Katoh, A. Buecker, B. Fausten, A. J. Wiethoff, J. E. Wildberger, P. Haage, E. C. Parsons, Jr., R. M. Botnar, P. B. Graham, M. Vettelschoss and R. W. Gunther; "Molecular MR imaging of human thrombi in a swine model of pulmonary embolism using a fibrin-specific contrast agent", Invest Radiol 2007, 42(8) 586-95. C. P. Stracke, M. Katoh, A. J. Wiethoff, E. C. Parsons, P. Spangenberg and E. Spuntrup; "Molecular MRI of cerebral venous sinus thrombosis using a new fibrin-specific MR contrast agent", Stroke 2007, 38(5) 1476-81.

R. J. J. H. M. Miserus, M. V. Herias, L. Prinzen, M. B. I. Lobbes, R. J. van Suylen, A. Dirksen, T. M. Hackeng, J. W. M. Heemskerk, J. M. A. van Engelshoven, M. J. A. P. Daemen, M. A. M. J. van Zandvoort, S. Heeneman and M. E. Kooi; "Molecular Imaging of Early Thrombus Formation using a Bimodal $\alpha_{2}$-antiplasmin-based contrast agent", JACC Cardiovasc Imaging 2009, In press.

A. Alonso, A. Della Martina, M. Stroick, M. Fatar, M. Griebe, S. Pochon, M. Schneider, M. Hennerici, E. Allemann and S. Meairs; "Molecular imaging of human thrombus with novel abciximab immunobubbles and ultrasound", Stroke 2007, 38(5) 1508-14. B. Wang, W. J. Zang, M. Wang, H. Ai, Y. W. Wang, Y. P. Li, G. S. He, L. Wang and X. J. Yu; "Prolonging the ultrasound signal enhancement from thrombi using targeted microbubbles based on sulfur-hexafluoride-filled gas", Acad Radiol 2006, 13(4) 428-33.

D. W. Piston; "Imaging living cells and tissues by two-photon excitation microscopy", Trends Cell Biol 1999, 9(2) 66-9.

A. Diaspro and M. Robello; "Two-photon excitation of fluorescence for three-dimensional optical imaging of biological structures", J Photochem Photobiol B 2000, 55(1) 1-8.

W. Denk, J. H. Strickler and W. W. Webb; "Two-photon laser scanning fluorescence microscopy", Science 1990, 248(4951) 73-6.

B. R. Masters and P. T. So; "Antecedents of two-photon excitation laser scanning microscopy", Microsc Res Tech 2004, 63(1) 3-11.

F. Pan and W. B. Gan; "Two-photon imaging of dendritic spine development in the mouse cortex", Dev Neurobiol 2008, 68(6) 771-8.

L. L. Cavanagh and W. Weninger; "Dendritic cell behaviour in vivo: lessons learned from intravital two-photon microscopy", Immunol Cell Biol 2008, 86(5) 428-38. 


\section{Chapter 2}

C. E. Brown and T. H. Murphy; "Livin' on the edge: imaging dendritic spine turnover in the peri-infarct zone during ischemic stroke and recovery", Neuroscientist 2008, 14(2) 139-46.

G. Eichhoff, M. A. Busche and O. Garaschuk; "In vivo calcium imaging of the aging and diseased brain", Eur J Nucl Med Mol Imaging 2008, 35 Suppl 1(S99-106.

K. Svoboda, W. Denk, D. Kleinfeld and D. W. Tank; "In vivo dendritic calcium dynamics in neocortical pyramidal neurons", Nature 1997, 385(6612) 161-5.

L. G. Ng, P. Mrass, I. Kinjyo, S. L. Reiner and W. Weninger; "Two-photon imaging of effector Tcell behavior: lessons from a tumor model", Immunol Rev 2008, 221(147-62.

M. Mellado and Y. R. Carrasco; "Imaging techniques: new insights into chemokine/chemokine receptor biology at the immune system", Pharmacol Ther 2008, 119(1) 24-32.

M. D. Cahalan and I. Parker; "Choreography of cell motility and interaction dynamics imaged by two-photon microscopy in lymphoid organs", Annu Rev Immunol 2008, 26(585-626.

S. L. Ashworth, R. M. Sandoval, G. A. Tanner and B. A. Molitoris; "Two-photon microscopy: visualization of kidney dynamics", Kidney Int 2007, 72(4) 416-21.

C. L. Phillips, V. H. Gattone, 2nd and S. M. Bonsib; "Imaging glomeruli in renal biopsy specimens", Nephron Physiol 2006, 103(2) p75-81.

M. van Zandvoort, W. Engels, K. Douma, L. Beckers, M. Oude Egbrink, M. Daemen and D. W. Slaaf; "Two-photon microscopy for imaging of the (atherosclerotic) vascular wall: a proof of concept study", J Vasc Res 2004, 41(1) 54-63.

R. T. A. Megens, M. G. A. o. Egbrink, M. Merkx, D. W. Slaaf and M. A. M. J. van Zandvoort; "Two-photon microscopy on vital carotid arteries: imaging the relationship between collagen and inflammatory cells in atherosclerotic plaques", Journal of Biomedical Optics 2008, 13(4) 044022-10.

R. T. Megens, M. G. Oude Egbrink, J. P. Cleutjens, M. J. Kuijpers, P. H. Schiffers, M. Merkx, D. W. Slaaf and M. A. van Zandvoort; "Imaging Collagen in Intact Viable Healthy and Atherosclerotic Arteries Using Fluorescently Labeled CNA35 and Two-Photon Laser Scanning Microscopy", Mol Imaging 2007, 6(4) 247-60.

W. Yu, J. C. Braz, A. M. Dutton, P. Prusakov and M. Rekhter; "In vivo imaging of atherosclerotic plaques in apolipoprotein E deficient mice using nonlinear microscopy", J Biomed Opt 2007, 12(5) 054008.

M. B. Lilledahl, O. A. Haugen, C. de Lange Davies and L. O. Svaasand; "Characterization of vulnerable plaques by multiphoton microscopy", J Biomed Opt 2007, 12(4) 044005. 
Imaging of atherosclerosis in murine arteries T. T. Le, I. M. Langohr, M. J. Locker, M. Sturek and J.-X. Cheng; "Label-free molecular imaging of atherosclerotic lesions using multimodal nonlinear optical microscopy", Journal of Biomedical Optics 2007, 12(5) 054007-10. 
Chapter 2 


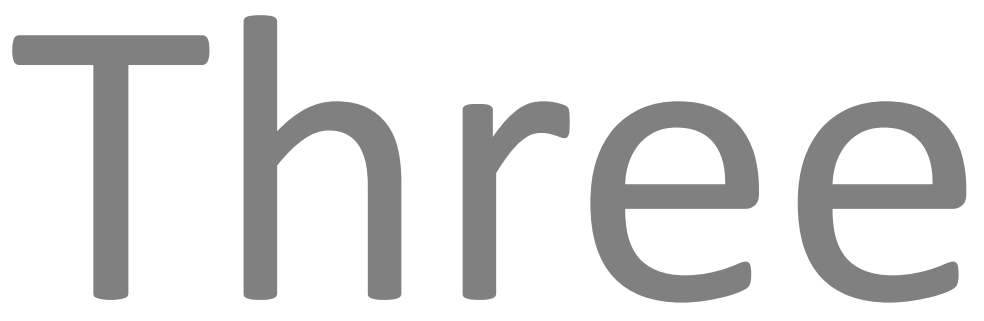

\section{Nanoparticles for optical molecular imaging of atherosclerosis}

Small 2009, 5(5) p544-557

K Douma *, L Prinzen *, DW Slaaf, CPM Reutelingsperger, EAL Biessen, TM Hackeng, MJ Post, MAMJ van Zandvoort

* Authors contributed equally. 


\section{Chapter 3}

\section{Abstract}

Molecular imaging contributes to future personalized medicine dedicated to treat cardiovascular disease, the leading cause of mortality in industrialized countries. Endoscope-compatible optical imaging techniques would offer a stand-alone alternative and high spatial resolution validation technique to the clinically accepted imaging techniques in the (intravascular) assessment of vulnerable atherosclerotic lesions, which are predisposed to initiate acute clinical events. Efficient optical visualization of molecular epitopes specific for vulnerable atherosclerotic lesions requires targeting of high quality optical contrast-enhancing particles. In this Review, we provide an overview of both current nanoparticles for optical imaging and targeting ligands for optical molecular imaging of atherosclerotic lesions and speculate on their applicability in the clinical setting. 


\section{Introduction}

Cardiovascular disease (CVD) is the leading cause of death in industrialized countries and places a social and financial burden on Western society by contributing strongly to acute clinical events, such as myocardial infarction. Myocardial infarction typically results from exposure of the plaque's thrombogenic content upon rupture. It would be of great clinical relevance to non-invasively assess atherosclerotic plaques in the presymptomatic phase, including detection of vulnerable plaques and intraplaque hemorrhage, and to classify the plaque's stage of development, thus allowing stratification of patients-at-risk. Assessment of atherogenesis in the early stage of development would direct early treatment to impede this process. The majority of current clinical imaging platforms, including X-ray angiography and Computed Tomography (CT), focus on postsymptomatic detection of morphological alterations of the arterial wall that affect the lumen diameter. However, the assessment of plaque extent is obscured by outward remodeling and does not provide direct information on plaque vulnerability. Therefore, molecular imaging strategies are being developed for non-invasive diagnosis of coronary artery disease (CAD) at various stages. Most of these strategies are based on recognizing the stage-specific expression of molecules that allow binding of ligandconjugated nanoparticles (NPs) ${ }^{1}$, which can be detected with non-invasive clinical imaging platforms such as Magnetic Resonance Imaging (MRI), radionuclide-based imaging (PET, SPECT), CT and Ultrasound (US). The routine application of NPs is still far ahead, but it is an actively evolving field of research with high promises for clinical use.

The feasibility of molecular imaging studies is based on progress made in three scientific fields: (1) non-invasive imaging platforms, (2) molecular epitope recognition and ligand identification, and (3) design of high quality contrast agents. As far as the latter is concerned, significant progress is being made in the design of contrast-enhancing NPs for molecular imaging ${ }^{2-5}$, enabling detection of these NPs with clinical imaging platforms, such as MRI and PET/SPECT. However, these imaging platforms have either limited sensitivity or low spatial resolution and therefore lack the ability to detect sparsely expressed molecular epitopes. Moreover, discrimination between specific and nonspecific binding or even between bound and circulating NPs is often difficult. In con- 


\section{Chapter 3}

trast, optical techniques combine high sensitivity with high spatial resolution ( $\mu \mathrm{m}$ range, reviewed in ${ }^{6}$ ) and therefore have gained considerable attention as techniques that allow (sub)cellular localization of targeted hybrid NPs with additional luminescent properties.

In this review, we explore the feasibility of optical imaging techniques in combination with NPs in the visualization and identification of vulnerable plaques and discuss the potential clinical applications. Common for all optical imaging techniques is the limited penetration depth, which ranges from sub-mm to several $\mathrm{cm}$ (see section 2 ). The penetration depth depends on tissue type, as each type has different absorbance and scattering properties which depend on composition and density. In order to reduce tissue absorbance, the excitation and emission light should match the optical window, which is usually in the 700-1200 $\mathrm{nm}$ range ${ }^{7}$. Below $650 \mathrm{~nm}$, absorbance of light by hemoglobin increases, while water absorbs light above $1200 \mathrm{~nm}$. Scattering of light may also cause a reduction in penetration depth. Plaque tissue consists for a large part of cholesterol esters, which are known to scatter light more than other tissue components. Also calcifications are known to scatter light. In other words, plaque imaging is hampered by its scattering properties. This can partially be resolved by using excitation with long wavelengths, and by using near infrared NPs.

Several optical imaging techniques are discussed. Two-photon laser scanning microscopy (TPLSM) allows fluorescence imaging at larger penetration depths than singlephoton techniques, such as confocal microscopy. Optical coherence tomography (OCT) is the optical equivalent of ultrasound, in which the backscatter of emitted light is captured into an image. Raman micro-spectroscopy (RMS) uses the vibration of chemical bonds to identify molecular composition. In photoacoustic tomography (PAT) light is converted into acoustic signals. These techniques will be more extensively discussed in section 2. This review further provides an overview of the physicochemical properties of five NPs for optical imaging that have been utilized in in vitro and in vivo studies in section 3. Additionally, in section 4 various possible targeting strategies are provided that ensure homing of the presented NPs to (vulnerable) atherosclerotic lesions. Subsequently, multi-modal NPs are presented that enable (sequential) visualization of pathological processes with complementary imaging platforms in section 5. Finally, we con- 
sider the clinical perspectives of the presented NPs for optical imaging in the Summary and Outlook section.

\section{Optical Imaging Platforms for Cardiovascular Research}

Optical imaging techniques offer a spatial resolution superior to conventional clinical imaging platforms and enable simultaneous visualization of multiple molecular epitopes. With optical imaging techniques, contrast can be generated by detecting (differences in) fluorophore emission spectra (fluorescence microscopy and derivatives), fluorescence lifetime (Fluorescence Lifetime Imaging, FLIM), polarization (polarization microscopy), molecular resonances (Raman Micro-Spectroscopy, RMS), backscattering (Optical Coherence Tomography, OCT) or photoacoustic waves (PhotoAcoustic Tomography, PAT). Due to the limited penetration depth of light, non-invasive whole-body animal luminescence imaging is confined to superficial or subcutaneous events, whereas exposure of tissue is mandatory in other situations, for example atherosclerosis. However, with progress made in (near) real-time endoscope-compatible microscopic systems, including Multi-Photon Laser Scanning Microscopy (MPLSM) ${ }^{8,9}, \operatorname{RMS}^{10}$, $\mathrm{OCT}^{11,12}$, and PAT ${ }^{13}$, state-of-the-art intravascular and real-time optical examination of the morphology of atherosclerotic plaques is within reach. Additionally, targeted NPs for optical imaging would allow assessment of the molecular expression patterns of these lesions (see Table 1 for the specifications of the optical imaging platforms).

Two-Photon Laser Scanning Microscopy (TPLSM) is the most frequently encountered version of MPLSM and is based on the non-linear absorption of two near-infrared (NIR) photons ${ }^{14}$, i.e. photons with wavelengths ranging $700-1000 \mathrm{~nm}$. The probability of excitation depends on the spatial and temporal overlap of two NIR photons at the excitable molecule. Even then absorption cross sections are extremely small and consequently excitation only occurs effectively at the objective's focal zone, the probability rapidly decreasing away from the focus. The absence of out-of-focus absorption and emission allows optical slicing and additionally limits photobleaching and-damage, allowing vital imaging of intact tissue. Furthermore, NIR photons have a wavelength approximately double that of traditional fluorescence microscopy and are thus less scattered, resulting in increased penetration depths up to $250 \mu \mathrm{m}$, the exact value strongly depending on 


\section{Chapter 3}

tissue characteristics. In cardiovascular research, TPLSM has primarily been applied ex vivo $^{15-17}$, but in vivo applications are within reach due to recent developments in imaging speed and optical detection efficiency.

Raman (Micro-)Spectroscopy (RMS) is based on detecting vibrational alterations of chemical bonds in molecules upon interaction with monochromatic light and hence provides detailed information on the molecular composition of tissue. For example, ex vivo RMS allows quantification of cholesterol content in arterial walls ${ }^{18}$ and recognition of atherosclerotic vasculature ${ }^{19,20}$. Furthermore, in vivo studies have shown that RMS is suitable for characterizing arterial wall composition ${ }^{10}$ and is applicable in tumor molecular imaging ${ }^{21}$.

Optical Coherence Tomography (OCT) utilizes the echo time delay and intensity of backscattered light to create cross-sectional images of sub-surface tissue structures. The penetration depth of $\sim 2 \mathrm{~mm}$ is 10 -fold larger than that of TPLSM; whereas the spatial resolution is 10 -fold lower. OCT allows ex vivo intravascular imaging and characterization of plaque morphology ${ }^{22-24}$ and in vivo visualization of vulnerable atherosclerotic plaques in mice ${ }^{25}$ and morphology in human coronary atherosclerotic plaques ${ }^{26,27}$.

In PhotoAcoustic Tomography (PAT), ultrasonic sources are generated by absorption of pulsed light. Though the application of PAT to cardiovascular disease is in its infancy, PAT allows vascular imaging in vivo with ${ }^{28,29}$ and without ${ }^{30,31}$ exogenous contrast agents. More interestingly, with intravascular PAT atherosclerotic plaques can be identified and characterized ex vivo ${ }^{32,33}$, which holds great promise for in vivo applications.

Taken together, RMS and OCT allow in vivo assessment of the molecular and structural changes of the atherosclerotic arterial wall. Examples of the in vivo application of TPLSM and PAT in visualizing atherosclerosis are currently unavailable in scientific literature, however, the various in vitro applications have shown their feasibility in atherosclerosis research. NPs for optical imaging, targeted towards molecular epitopes specifically expressed in each stage of plaque development, will increase the sensitivity in the microscopic evaluation of the plaque's stage of development. 
Table 1. Optical imaging platforms suitable for intravascular applications.

\begin{tabular}{|c|c|c|c|c|c|}
\hline $\begin{array}{l}\text { Optical plat- } \\
\text { form }\end{array}$ & Contrast & $\begin{array}{l}\text { Penetration } \\
\text { depth [a] }\end{array}$ & \multicolumn{2}{|c|}{$\begin{array}{l}\text { Spatial resolution } \\
{[\mu \mathrm{m}]}\end{array}$} & $\begin{array}{l}\text { Temporal resolution } \\
\text { [fps] [b] }\end{array}$ \\
\hline \multirow[t]{2}{*}{ TPLSM } & Spectral & $\sim 250[\mu \mathrm{m}]$ & Axial & $<1$ & up to 50 \\
\hline & & & Lateral & $<1$ & \\
\hline \multirow[t]{2}{*}{ OCT } & $\begin{array}{l}\text { Echo delay \& back- } \\
\text { scatter }\end{array}$ & $1-2[\mathrm{~mm}]$ & Axial & $\sim 20$ & 30 \\
\hline & & & Lateral & $2-4$ & \\
\hline \multirow[t]{2}{*}{ RMS } & $\begin{array}{l}\text { Molecular reso- } \\
\text { nance }\end{array}$ & $450[\mu \mathrm{m}]$ & Axial & - & 1 \\
\hline & & & Lateral & 25 & \\
\hline \multirow[t]{2}{*}{ PAT } & $\begin{array}{l}\text { Laser-induced ultra- } \\
\text { sound }\end{array}$ & several $[\mathrm{cm}]$ & Axial & 25 & 1 \\
\hline & & & Lateral & 100 & \\
\hline
\end{tabular}

[a] Penetration depth: maximal depth from which an adequate signal may be acquired.

[b] Temporal resolution: maximal frame-rate (in frames per second, $\mathrm{fps}$ ) that may be attained.

\section{Nanoparticles for Optical Imaging in Cardiovascular Research}

For in vivo molecular imaging, NPs for optical imaging require good physicochemical properties for efficient optical detection and functionalization by targeting ligands. In addition to NP size and shape, modifications in surface structure, e.g. by altering surface charge and attachment of bio-molecules, affect the pharmacokinetics (reviewed in ${ }^{34}$ ). Numerous covalent, amphiphilic and electrostatic strategies have been developed to coat NPs for optical imaging and adapt circulation half-life, organ accumulation and excretion pathways, but may have opposing effects on the NP luminescent properties. For example, organic coating of quantum dots (QDs) increases hydrophilicity, chemical stability and luminescent properties ${ }^{35}$, whereas covalent functionalization of carbon nanotubes (CNTs) decreases the luminescent properties ${ }^{36}$. Although NP surface chemistry affects their in vivo behavior and luminescent properties, an elaborate discussion on this matter extends the scope of this paper. We therefore refer to ${ }^{37}$ for additional information. Note that the physicochemical properties in Table 2 apply for watersoluble NPs for optical imaging without targeting ligands. 

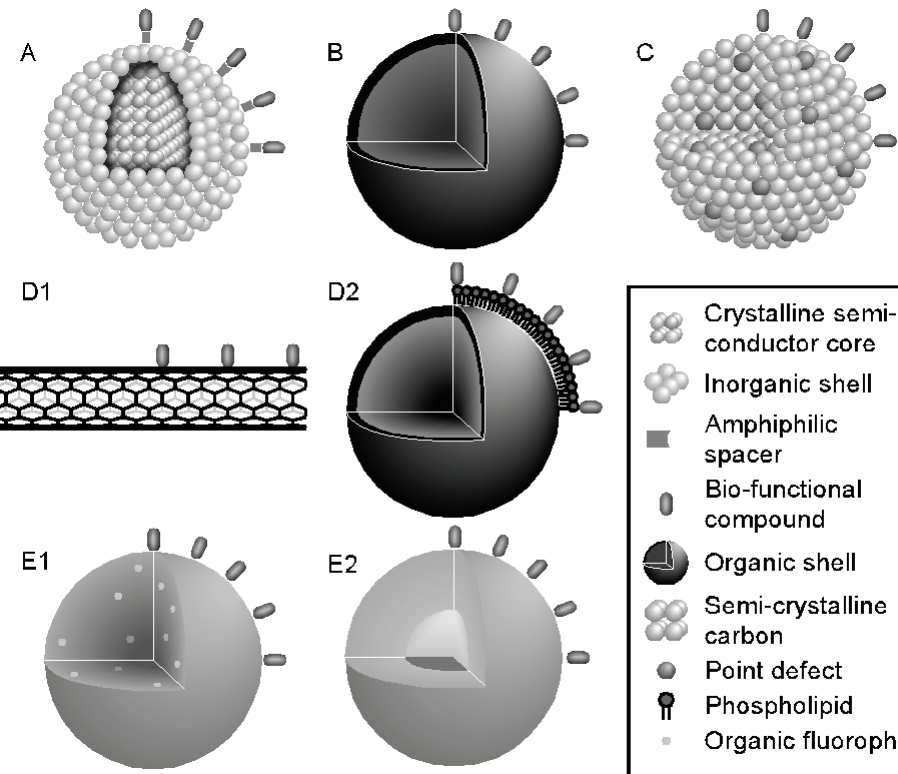

Crystalline semiconductor core Inorganic shell Amphiphilic spacer Bio-functional

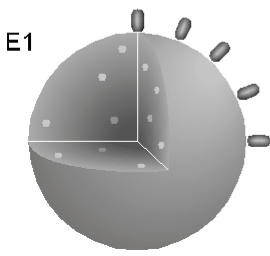
compound

d) Organic shell Semi-crystalline carbon

- Point defect

if Phospholipid

Organic fluorophore

Figure 1. Schematic overview of the composition of A) quantum dot, B) gold nanoparticle, C) diamond nanoparticle, D1) carbon nanotube, D2) carbon dot (C-Dot), E1) silica nanoparticle with encapsulated organic fluorophores, E2) silica nanoparticle with fluorescent core. Several chemical methods for surface binding of bio-active compounds are mentioned in the text. See Table 2 for spatial dimensions. Color figure on page 180.

The NPs for optical imaging described here (see Figure 1 for graphical representations and Table 2 for their main physicochemical properties) are relatively new, but some have already been tested in vivo in preclinical disease models. Quantum dots (QDs) and gold NPs (AuNPs) have proven extremely useful in molecular imaging with optical methods, including TPLSM, OCT and PAT, but presumably have limitations with respect to long term biocompatibility. Three recently synthesized NPs, diamond (DNP), carbon (CNP) and silica (SNP), theoretically have a better biocompatibility and have been tested in vitro for imaging purposes and were shown to have characteristics highly suitable for in vivo optical imaging. These five NPs will be discussed below.

The physical principles behind the luminescence from QDs, AuNPs and CNPs are beyond the scope of this paper. Therefore light emitted by these NPs is simply referred to as luminescence. 


\section{Quantum Dots}

Quantum dots (QDs) are nanometer-sized, semiconductor crystals (Figure 1A) that offer superior photophysical properties over organic dyes ${ }^{38,39}$, including high quantum yields 40, broad excitation spectra and narrow emission spectra and high photostability ${ }^{41,42}$ (Table 2).

Importantly, they allow efficient two-photon excitation, which together with the aforementioned advantageous properties makes them suitable for TPLSM imaging deep in scattering tissue. Moreover, the size-dependent emission wavelength ${ }^{43}$ allows simultaneous visualization of differently sized QD with a single-wavelength excitation source. The photophysical properties of QDs are strongly dependent on regularity in core structure and size-distribution and their synthesis therefore requires stringent conditions ${ }^{44,}$ ${ }^{45}$. The QD quantum yield can reach 70\% by encapsulating the QD core with an inorganic shell to obtain core/shell QDs ${ }^{46,47}$. The organic shell also reduces desorption and subsequent oxidation of the toxic core components ${ }^{48}$. Moreover, additional organic coating of core/shell QDs provides both water-solubility and increased core/shell stability ${ }^{35}$, which are required for in vivo applications.

In mice, QDs are generally cleared from the blood by accumulation in organs of the reticuloendothelial system (RES) and in some cases also the kidneys ${ }^{49,50}$, although this may vary depending on the surface chemistry and ligands attached. However, QDs are usually not excreted through urine or feces ${ }^{50,51}$, even for months after intravascular administration, and are therefore assumed to reside in these organs. It is assumed that eventually the accumulated QDs will chemically degrade and release their cytotoxic components and are therefore considered to be clinically unsafe. Therefore, several methods were recently developed to produce QDs that are composed of non-toxic components ${ }^{52,53}$, are encapsulated in a silica-shell to increase biocompatibility ${ }^{54}$, and are cleared by the kidneys ${ }^{49}$. These new developments will potentiate the use of QD in future clinical applications ${ }^{55}$.

Targeted QDs are obtained by covalently binding ligands to the shell-surface ${ }^{56}$ or via non-covalent strategies ${ }^{57}$, including the streptavidin-biotin bond ${ }^{58}$. The latter is frequently used in biomedical research. Thus far, QDs have been applied to cancer re- 


\section{Chapter 3}

search in animals, including sentinel node mapping ${ }^{59,60}$ and in vivo molecular imaging of tumors ${ }^{57,61}$. The application of (targeted) QDs to cardiovascular research is advancing. QDs have been used to visualize ex vivo the expression of the oxLDL receptor CD36 62, vascular adhesion molecule-1 (VCAM-1) and intracellular adhesion molecule-1 (ICAM-1) ${ }^{63}$, the angiogenic marker CD13 ${ }^{15}$, and phosphatedylserine (PS) exposing cells ${ }^{64}$. Recently, ICAM-, VCAM-, as well as platelet endothelial cell adhesion molecule (PECAM) - conjugated QDs have been used for simultaneous targeting and imaging of the endothelial phenotype in retinal vasculature ${ }^{65}$. In addition, the proteolytic activity of matrix metalloproteinases (MMPs) in atherosclerotic plaques, contributing to a mechanically unstable phenotype (see section 4), can be visualized using QD-based biosensors ${ }^{66}$, which were successfully applied in vivo in tumors ${ }^{67}$. However, the approaches mentioned above require external excitation, often implying exposure of the tissue of interest. By chemical excitation of infrared emitting QDs via bioluminescence resonance energy transfer (BRET) ${ }^{68}$, true non-invasive optical imaging may be realized.

The examples above show that QDs are highly suitable for optical imaging in cardiovascular research. However, besides the optical properties, the local contrast enhancing effects of targeted NPs are also dependent on ligand-receptor interactions. Various studies have shown that QDs allow quantification of ligand-receptor binding kinetics ${ }^{69}$ and monitoring of receptor-mediated endocytosis ${ }^{41,58}$. Therefore, QDs may be applicable to in vivo probing of binding affinity and subsequent processing of ligands specific for atherosclerosis-related molecular epitopes.

\section{Gold Nanoparticles}

Gold particles are used extensively in electron microscopy ${ }^{70}$. Recent progress in the synthesis of gold nanoparticles (AuNPs, Figure 1B) has yielded highly luminescent NPs with emissions ranging from $400 \mathrm{~nm}^{71}$ to $1200 \mathrm{~nm}^{72}$. The near-infrared emission makes them suitable for optical spectral imaging of biological tissues, since tissue absorption at these wavelengths is limited. AuNPs require an adequate protecting and stabilizing organic shell for their luminescence and display relatively narrow excitation and broad emission spectra with a quantum yield up to $40 \%{ }^{71}$. Similar to QDs, the emission spectra of AuNPs are size-dependent ${ }^{73}$. Moreover, AuNPs are strongly luminescent upon 
two-photon absorption ${ }^{74}$ and display specific surface scattering upon excitation with the appropriate wavelength ${ }^{75}$, indicating that AuNPs can be visualized by both TPLSM and OCT. Moreover, excitation with NIR photons induces ultrasonic waves, which allow detection with PAT $^{29}$ (see Table 2).

In vitro, organically stabilized AuNPs are internalized via endocytosis ${ }^{76,77}$ without affecting cell viability ${ }^{77,78}$. In vivo, AuNPs predominantly accumulate in the liver and spleen of mice ${ }^{79}$, do not initiate (hepatic) toxicity ${ }^{80}$, and are slowly excreted via the feces ${ }^{81}$. AuNPs are clinically approved for rheumatoid arthritis ${ }^{82,83}$ and are considered safe ${ }^{84}$, although several studies showed a correlation between AuNP therapy and lung disease 85,86

Covalent attachment of amine ${ }^{87}$ and thiol ${ }^{88}$ groups to the AuNP surface allow easy functionalization with amino acids ${ }^{89}$ and proteins ${ }^{90}$ for targeted delivery, e.g. to atherosclerosis-related markers. Moreover, direct capping of the AuNP surface with certain amino acids yields stable, water-dispersible and functionalized particles ${ }^{89,91}$.

Agglutination of AuNPs enhances their detection with reflectance microscopy, including $\mathrm{OCT}^{92}$, thus allowing in vitro visualization of cancer cells ${ }^{75,76}$. This phenomenon can be exploited in molecular imaging since surface-expressed markers spatially coincide, thereby bringing targeted AuNPs in close contact with each other. Additionally, TPLSM allows visualization of non-targeted AuNPs in vivo ${ }^{93}$ and identification of AuNP-labeled cancer cells in tissue phantoms targeted by AuNPs ${ }^{94}$.

\section{Diamond Nanoparticles}

Diamond nanoparticles (DNPs) represent a potentially ideal optical label, combining efficient scattering properties (i.e. they can be used for OCT) ${ }^{95}$, bright ${ }^{96}$, and photostable fluorescence ${ }^{96}$, low cytotoxicity ${ }^{97}$, and chemical stability (Table 2). Fluorescence arises from so-called point defects (Figure 1C) ${ }^{98}$, which are initiated by high-energy irradiation ${ }^{99}$. Each of the defect centers has a fluorescence brightness comparable to a single organic fluorophore, enabling detection of individual DNPs by fluorescence microscopy ${ }^{97}$, including TPLSM ${ }^{100}$. However, current DNPs have broad emission spectra ${ }^{96}$, which significantly hampers multicolor imaging. For functionalization, DNPs are chemically modified to yield carboxylated DNPs for the covalent attachment of bioactive 


\section{Chapter 3}

ligands ${ }^{96,101}$. Recent progress in the dispersion of DNPs in aqueous media has facilitated their use in physiological solutions ${ }^{102}$.

Preliminary results yielded DNPs as non-toxic in vitro ${ }^{97}$ as well as in vivo ${ }^{103}$. Long term follow-up studies on DNP toxicity however are currently lacking.

The favorable photophysical and potential pharmacodynamic characteristics of DNPS show great promise for biological applications, yet preclinical research on DNPs is still in its infancy. In vitro, DNPs were shown to enable single-particle tracking in the cytoplasm of HeLa cells ${ }^{96}$ and detection of growth hormone receptor expression ${ }^{101}$.

\section{Carbon Nanoparticles}

Carbon nanoparticles (CNPs), which include carbon nanotubes (CNTs, Figure 1D1) and carbon dots (or C-Dots ${ }^{104}$, Figure 1D2), may offer a less cytotoxic alternative to the aforementioned quantum dots. CNTs and C-Dots are photostable ${ }^{104,105}$, but display relatively broad - excitation wavelength dependent - emission spectra (Table 2) ${ }^{105,}{ }^{106}$. Therefore, in contrast to QDs, multi-color TPLSM imaging of CNPs in tissue is difficult. Non-aggregated CNTs display an inherent NIR luminescence with a tube-length dependent quantum yield ${ }^{107}$, reaching values up to $10 \%{ }^{108}$. C-Dots, however, require a large surface-to-volume ratio and surface stabilization with organic polymers, such as PEG1500, to become luminescent ${ }^{105}$. Several strategies were developed to obtain individualized and hydrophilic CNTs, including non-covalent functionalization with proteins ${ }^{109}$, dendrimers ${ }^{110}$ and phospholipids ${ }^{111}$. CNPs are internalized via endocytosis in vitro 105, 112 , but both CNTs and C-Dots display no acute toxicity ${ }^{104,113}$. However, long-term effects of CNPs on cell viability strongly depend on surface-bound (bio-)molecules and dose ${ }^{112,114}$ and traces of metal catalysts used during synthesis ${ }^{113}$. In addition, CNTs may penetrate the nuclear membrane physically ${ }^{115}$ and elicit DNA damage through ROS generation ${ }^{116}$. After intravenous administration of CNTs, no pathological abnormalities were found in the liver, kidneys and other organs ${ }^{117}$. In rodents, CNTs are cleared by the kidneys ${ }^{118}$, whereas excretion routes of C-Dots are currently unknown. CNPs may be functionalized for molecular imaging by coating with ligand-bound phospholipids ${ }^{119}$ and amino-terminated dendrimers ${ }^{110}$. 
Until now, few studies have utilized CNPs for in vivo optical imaging. Nevertheless, the NIR and Raman signals from CNTs were detected in the blood pool of Drosphilia melanogaster ${ }^{106}$ and in subcutaneously implanted tumors in mice ${ }^{21}$, respectively. These preliminary results indicate the potential of TPLSM in visualizing CNPs in biological tissue.

\section{Silica Nanoparticles}

Fluorescent silica nanoparticles (SNPS) are synthesized by trapping fluorophores in a mesoporous silica matrix (Figure 1). The spectral properties thus depend on the entrapped fluorophores and can therefore not be specified. The quantum yield has an optimum since both a low concentration and too high concentration (inducing selfquenching ${ }^{120}$ ) will affect SNP brightness ${ }^{121}$. The brightness of dye-doped SNPs (Figure 1E1) additionally suffers from dye-leakage from the silica pores, which also hampers accurate assessment of the spatial distribution of SNPs by optical imaging modalities. The leakage can be reduced by either covalent ${ }^{42}$ or electrostatic interactions ${ }^{120}$ between the fluorophore and the silica precursors, or by increasing the size of the dye molecule ${ }^{120}$, or by stabilizing the fluorophore-doped silica core with a dye-free silica shell ${ }^{42,120}$ (Figure 1E2). Core/shell SNPs exhibit a relatively high brightness, but still display an approximately 3-fold lower luminescence intensity than QDs ${ }^{42}$.

Reactive silica precursors enable chemical modifications of the SNP surface. For example, maleimide and iodoacetyl groups can be introduced ${ }^{122}$ and amino groups ${ }^{123}$ can be ionized to enable (covalent) attachment of bioactive ligands.

In vitro experiments show cytotoxic responses to SNPs. Upon internalization by endocytosis ${ }^{124}$, SNPs impair proliferative activity, stimulate the release of the inflammation marker interleukin-8, and reduce cell viability in vitro in a dose- and time-dependent manner ${ }^{125-127}$. In mice, however, intravenous administration of colloidal core/shell SNPs at biologically relevant concentrations do not induce acute or chronic responses, even 60 days post-injection ${ }^{128}$.

Several in vitro fluorescence microscopy studies have shown that SNPs allow peripheral labeling of cancer cells by targeting folate receptors ${ }^{42,129}$. In addition, antibodyconjugated SNPs allow multi-target monitoring of bacteria species ${ }^{130}$. 


\section{Detection of nanoparticles for optical imaging}

For efficient detection, NPs for optical imaging should have favorable luminescence properties, i.e. high quantum yield, broad excitation spectrum and narrow emission spectrum that matches the optical window of tissue. The presented NPs for optical imaging share the ability to be detected with TPLSM (Table 1). However, the $250 \mu \mathrm{m}$ penetration depth of TPLSM in biological tissue limits its clinical application to early atherosclerotic lesions. The penetration depth of OCT and PAT are 1 and 2 orders larger, respectively, and may therefore be preferred over TPLSM when greater depths should be attained, i.e. intraplaque visualization of developed lesions.

Table 2. Physicochemical properties of water-soluble non-targeted nanoparticles for optical molecular imaging.

\begin{tabular}{|c|c|c|c|c|c|c|}
\hline & Quantum Dot & Gold & Diamond & $\begin{array}{l}\text { Carbon } \\
\text { dot }\end{array}$ & Carbon tube & Silica \\
\hline Quantum yield & $<70 \%{ }^{46,47,131}$ & $<40 \%{ }^{71}$ & $\sim 50 \%{ }^{96}$ & $\sim 10 \%{ }^{105}$ & $<10 \%{ }^{108,132}$ & $\begin{array}{l}\text { Fluorophore \& } \\
\text { concentration } \\
\text { dependent }\end{array}$ \\
\hline $\begin{array}{l}\text { Fluorescence } \\
\text { excitation } \\
\text { spectrum [nm] }\end{array}$ & $>200$ [a] & $300 \sim 450$ [a] ${ }^{71}$ & $\begin{array}{l}450 \sim 550 \\
\text { [a] }^{97,133,134}\end{array}$ & $\begin{array}{l}450 \sim 650 \\
{[\mathrm{a}]^{105}}\end{array}$ & Unknown & $\begin{array}{l}\text { Fluorophore } \\
\text { dependent }\end{array}$ \\
\hline $\begin{array}{l}\text { Fluorescence } \\
\text { emission spectrum } \\
{[\mathrm{nm}]}\end{array}$ & 30 [b] & $\sim 60[\mathrm{~b}]$ & $\begin{array}{l}\sim 100[\mathrm{~b}]^{96,} \\
133\end{array}$ & $\begin{array}{l}\sim 100[\mathrm{~b}] \\
105\end{array}$ & $\begin{array}{l}\sim 500[\mathrm{a}]^{106,114,} \\
117\end{array}$ & $\begin{array}{l}\text { Fluorophore } \\
\text { dependent }\end{array}$ \\
\hline $\begin{array}{l}\text { Hydrodynamic } \\
\text { diameter [nm] }\end{array}$ & $<20$ & $1-50$ & $35-100$ & $<5$ & $\begin{array}{l}\sim 1 \mathrm{~nm}(\varnothing) ; 500 \\
\text { (length) }\end{array}$ & $>15$ \\
\hline Blood clearance & $\begin{array}{l}\text { Predominantly } \\
\text { spleen \& liver }\end{array}$ & $\begin{array}{l}\text { Predominantly } \\
\text { spleen \& liver }\end{array}$ & Unknown & Unknown & Kidneys & $\begin{array}{l}\text { Spleen, liver \& } \\
\text { lung }{ }^{128}\end{array}$ \\
\hline Excretion & None & $\begin{array}{l}\text { Slowly via feces } \\
81\end{array}$ & Unknown & Unknown & Urine $^{118}$ & $\begin{array}{l}\text { Urine and feces } \\
135\end{array}$ \\
\hline Optical platform & TPLSM & TPLSM, OCT, PAT & $\begin{array}{l}\text { TPLSM, } \\
\text { OCT }\end{array}$ & TPLSM & TPLSM & TPLSM \\
\hline
\end{tabular}

[a] Total bandwidth; [b] Full-Width-at-Half-Maximum (FWHM) 


\section{Targeting nanoparticles to atherosclerotic lesions}

The application of molecular imaging in assessing vulnerable atherosclerotic plaques is a challenging task, since these plaques are highly complex and constantly evolving structures. Consequently, these lesions display numerous molecular targets, expressed either at the endothelial surface or subendothelially by cells and extracellular matrix in the vascular wall.

The American Heart Association (AHA) has recognized six states of plaque development in humans, divided in precursor and advanced types (see Figure 2). Precursor lesions (types I, II, III) are asymptomatic and do not narrow the lumen ${ }^{136}$; whereas advanced lesions (types IV, V, VI) are hemodynamically and clinically more relevant since they may lead to ischemic attacks ${ }^{137}$. Type I plaques are characterized by endothelial activation, which is accompanied by luminal expression of various adhesion molecules ${ }^{138}$; these include intercellular (ICAM), vascular (VCAM), and platelet endothelial (PECAM) cell adhesion molecules, and the lectin-like oxLDL receptor LOX-1. These adhesion molecules initiate the accumulation of macrophages and lipids in the vascular wall, causing gradual progression to type II lesions. Type III lesions contain extracellular lipid and apoptotic cells ${ }^{138}$ and typically show extracellular matrix-degrading enzymes, such as matrix metalloproteinases (MMPs) and cathepsins ${ }^{138}$. In type IV lesions the necrotic core develops, as large amounts of extracellular lipid accumulate in the intima. Capillaries surround the lipid core, mostly in the shoulder of the plaque, and calcifications may also be present. Macrophages, foam cells, and lymphocytes are mainly present in the periphery of the lesion, most notably in the shoulders of the plaque. Type $\mathrm{V}$ lesions develop when the subendothelial layer grows into more collagen-rich tissue, forming the fibrous cap. More and larger capillaries are present in these lesions. Type VI lesions closely resemble type IV and V lesions, but have had disruptions of the lesion surface, hematoma or hemorrhages, with subsequent thrombus formation. It is important to stress that vulnerable plaques do not necessarily coincide with rupture-prone plaques. More accurately, vulnerable plaques are at risk for thrombotic complications and consequent rapid progression of plaque instability, as was proposed by Naghavi et al. ${ }^{139}$. 


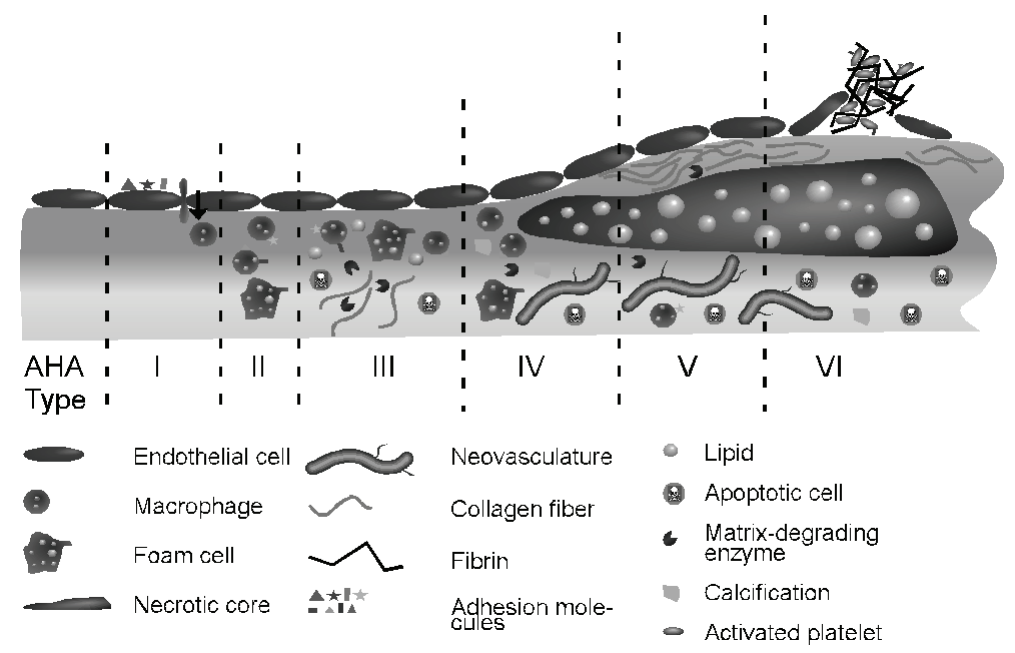

Figure 2. Schematic representation of human plaque progression. The different types of plaque morphologies are not necessarily successive and not all plaques progress to ruptured plaques (AHA type $\mathrm{VI}$ ). Color figure on page 180 .

Each stage of plaque development displays specific features, such as inflammation, activated endothelium, accumulated lipid and macrophages, collagen-rich fibrous cap, angiogenic activity, apoptosis and thrombi. Each of these features is associated with specific molecular targets that may be used for molecular imaging. Available targets and corresponding ligands, which have been used in clinical and pre-clinical studies, are mentioned in Table 3. The NPs in Table 3 are not necessarily NPs for optical imaging; however, the ligands mentioned in Table 3 can be combined with the NPs for optical imaging in section 3, to generate particles for specific and high-resolution visualization of vulnerable plaques using (endoscope-based) optical imaging techniques. Optical techniques described in Table 3 either require exposure of the tissue of interest or served as an ex vivo validation tool. Optical imaging techniques are currently being developed that would allow in vivo, endoscopic-based imaging (see section 2 ).

A distinction should be made between luminal (such as CD13 ${ }^{15,140}$ ) and sub-endothelial targets (such as apoptotic cells in atherosclerotic plaques ${ }^{141}$ ). For sub-endothelial targets the hydrodynamic size of the NP should be considered, as the particle has to translocate through the endothelial layer. Furthermore, the permeability of the endothelium should be taken into account, as plaque endothelium is leakier than healthy endothe- 
lium, allowing translocation of larger NPs. In advanced plaques, NPs may also reach the intraplaque targets through the vasa vasorum, an adventitial vascular network that expands into the lesion during plaque growth. 
Chapter 3

Table 3- CH3b 
Nanoparticles for optical molecular imaging of atherosclerosis

Table 3- CH3b 
Chapter 3

Table 3-CH3b 


\section{Hybrid nanoparticles for multimodal imaging}

Clinically approved imaging techniques allow non-invasive evaluation of the localization of targeted contrast enhancing NPs, yet lack the sensitivity and resolution to prove target specificity. Therefore, high spatial resolution validation of non-invasive molecular imaging is mandatory and requires NPs suitable for multimodal imaging. Hybrid NPs can be detected by optical imaging methods as well as by non-invasive clinical imaging methods. These hybrid NPs may also be linked to the ligands in table 3, for imaging of atherosclerosis-related molecular markers. In such a set up, a combination of noninvasive imaging for macroscopic detection, followed by invasive optical imaging, allows determination of distribution and exact location. Hybrid NPs developed so far are mostly decorated with MRI and fluorescent labels. Of the hybrid NPs currently available, few have been applied in vivo.

Hybrid lipidic structures, such as liposomes and micelles, are composed of natural amphiphilic lipids and are therefore highly biocompatible. They can be easily formulated and adjusted for various purposes. The maximum payload of added moieties, e.g., the ligand or contrast agent, depends on the size of the liposomes, but may be thousands per liposome. The size of liposomes is often larger than $100 \mathrm{~nm}$, which is large compared to the NPs discussed in previous paragraphs. In recent decades, though, liposomes have been investigated extensively as drug carriers ${ }^{173,174}$ and two liposomal cytostatic formulations have been approved for clinical use so far ${ }^{175}$. Incorporation of contrast agents allows their application in imaging purposes, e.g. for in vivo MRI detection of intimal thickening ${ }^{176}$. When contrast agents with different functionalities are incorporated in the lipid bilayer, dual detection with for instance MRI and fluorescence microscopy is enabled. Hybrid liposomes have been developed to specifically target and visualize the expression of E-selectin ${ }^{177}$ and apoptotic cells ${ }^{178} \cdot \alpha_{v} \beta_{3}$ targeted liposomes with surface-conjugated RGD have been used to detect angiogenic activity in tumors ${ }^{179}$ and in atherosclerotic plaques ${ }^{159}$ by in vivo MRI and subsequent ex vivo validation with fluorescence microscopy ${ }^{179}$. Bimodal micelles targeting the macrophage scavenger receptor (MSR) by surface-bound anti-MSR antibodies visualize the uptake of macro- 


\section{Chapter 3}

phages in atherosclerotic plaques of mice with in vivo $\mathrm{MRI}^{180}$, and by ex vivo fluorescence microscopy.

QDs may serve as a highly luminescent scaffold for MR probes, PET/SPECT tracers and CT contrast agents to produce multimodal imaging probes. The payload of additional contrast agents or ligands is estimated to be 10-30 per QD. QDs provided with a paramagnetic lipid-coating were applied to $M R$ and optical visualization of $\alpha_{v} \beta_{3}$-expression on the membranes of activated HUVECs ${ }^{181}$ or for PS exposure of apoptotic cells ${ }^{182}$. A different approach was chosen through silica-coated QDs with a hydrophobic phase between the QD surface and the silica-shell ${ }^{183}$ in order to incorporate hydrophobic contrast agents. The drawback of this approach is the rather tedious and stringent synthesis protocol to produce monodisperse NPs containing a single QD. The time-consuming synthesis makes these particles less suitable for PET/SPECT applications, due to decay of radioactive tracers.

A more flexible scaffold is provided by streptavidin-coated QDs, since the QD-surface can easily be functionalized by biotinylated moieties, including ligands and non-optical imaging probes. To further increase detectability, dendritic wedges with 8 DTPA or DOTA compounds were developed to incorporate paramagnetic gadolinium or radioactive PET/SPECT tracers, respectively ${ }^{184}$. Utilizing these dendritic structures, paramagnetic QDs were successfully applied for MR and optical imaging of apoptosis ex vivo using Annexin A5 ${ }^{64}$. Moreover, cNGR-coupled QDs were successfully utilized for in vivo optical detection of angiogenic activity after myocardial infarction ${ }^{15}$. Recently, a bimodal cNGR-coupled QD with a dendritic gadolinium wedge was shown to enable the detection of angiogenic activity in tumor-bearing mice by in vivo MRI and ex vivo TPLSM 140 .

A different type of hybrid NP is a QD containing a shell doped with paramagnetic manganese ${ }^{185}$. This NP was shown to be taken up by macrophages in vitro as shown by MRI and optical methods.

The mesoporous matrix of silica NPs and coatings allows the incorporation of probes with different functionality. By coating a luminescent ruthenium core with a silylated Gd complex, bimodal NPs were obtained for efficient luminescent and paramagnetic 
labeling of monocytes in vitro ${ }^{186}$, with the potential to extrapolate this to in vivo visualization of leukocyte accumulation in atherosclerotic plaques. Also, gadolinium oxide cores may be covered by a silica layer containing fluorophores ${ }^{187}$. In contrast to the previously discussed NPs, these PEGylated NPs were excreted mainly via the kidney when intravenously injected in mice, as was visualized in vivo with both fluorescence imaging and MRI. In addition, magnetite $\left(\mathrm{Fe}_{3} \mathrm{O}_{4}\right)$ can be coated with a silica shell, in which luminescent iridium is included, in order to visualize their passive ingestion by HeLa cells in vitro ${ }^{188}$.

In contrast to gadolinium, superparamagnetic compounds, such as iron oxides generate negative contrast with MRI. Several different iron oxide based multimodal nanoparticles have been developed, but only few have been applied in vivo so far. Magnetite particles, to which NIR fluorophores were conjugated ${ }^{189}$, were used to load macrophages for in vivo imaging applications. In addition, iron oxide particles covered with a phospholipidic layer containing fluorophores ${ }^{178}$ were developed to specifically visualize apoptotic cells in vitro. Also so-called nanosponges were developed in which the magnetic and luminescent characteristics of their components are maintained ${ }^{190}$. The in vivo experience with iron oxide based hybrids is still limited. Small $(3 \mathrm{~nm})$ iron oxide NPs coated with fluorescent cyanine 5.5, allowed visualization of atherosclerotic lesions in mice with in vivo MRI and ex vivo fluorescence microscopy ${ }^{191}$, apparently by virtue of their macrophages uptake. These results have promising implications for non-invasive assessment of inflammation, and vulnerability to rupture, of an atherosclerotic plaque.

Also, CNT conjugation to iron oxide nanoparticles resulted in a bimodal NP allowing visualization of the in vitro uptake by macrophages with MRI, fluorescence imaging and Raman scattering ${ }^{192}$. To increase biocompatibility, this NP was encapsulated in DNA.

\section{Summary and outlook}

In this paper, optical and hybrid contrast enhancing NPs were described that have been applied to in vitro and in vivo animal studies. As such, these particles may play a role in confirming (sub) cellular localization following non-invasive imaging in animal models. Due to fast developments, NPs may also find clinical applications in optical imaging techniques. The ideal NPs for optical molecular imaging in clinical use should bear the 


\section{Chapter 3}

following characteristics: (1) highly luminescent upon irradiation, (2) high binding affinity and specificity for the target, (3) resistance to chemical degradation and (4) clearance through kidney or liver upon intravenous injection, to prevent accumulation in vital organs. It should be kept in mind that the in vivo targeting behavior, as well as the level of toxicity, of the NPs highly depend on geometrical (i.e. NP size), biophysical (i.e. the availability of the target and hemodynamic forces), chemical (i.e. NP stability), and biological (i.e. ligand-target affinity and excretion pathway) parameters. As such, a thorough quantitative analysis of the in vivo bio-distribution and pharmacokinetics of each individual construct (i.e., NP and applied surface modifications) should be performed and should result in a well defined and uniform safety and efficacy profile prior to clinical use.

The relatively narrow emission spectra of QDs and AuNPs offer a valuable contribution to the research of complex and multifactorial diseases, including atherosclerosis, since multiple molecular targets may be visualized simultaneously. However, the current commercially available QDs consist of heavy metals such as cadmium, and are not likely to be suitable for clinical use, despite encapsulation of the core. CNPs offer a potentially non-toxic alternative to QDs, but display a low quantum yield and broad emission spectrum, both hampering multi-color imaging in vivo. AuNPs, DNPs and SNPs combine high luminescence with a non-toxic potential in vivo, although it is not yet known whether DNPs are excretable. Therefore, based on current knowledge on toxicity and optical properties of the presented NPs, AuNPs and SNPs are the most promising NPs for clinical use in optical molecular imaging of atherosclerosis. The use of AuNPs is clinically approved in treatment of rheumatoid arthritis; ${ }^{82,83}$ however, both AuNPs and SNPs have been poorly characterized for in vivo imaging applications at this moment.

Many of the presented NPs for optical imaging hold a dual-purpose character, i.e. they may be used as contrast agent in optical imaging as well as therapeutic agent to specifically disrupt processes that contribute to plaque destabilization. These processes include MMP expression by macrophages and angiogenic expansion of the vasa vasorum that penetrates the plaque. The use of NPs for optical imaging as therapeutic agent is still in initial phase of development, but several approaches can be recognized. First, surface binding or encapsulation of photosensitizers may employ QDs and SNPs, re- 
spectively, for targeted photodynamic therapy (PDT) of atherosclerotic lesions ${ }^{193-199}$. In photodynamic therapy, (indirect) illumination of the photosensitizers induces local formation of reactive oxygen species, which stimulates apoptosis of the surrounding cells. Second, AuNPs ${ }^{200}$ and CNTs ${ }^{201}$ convert absorbed radiation into heat ${ }^{200}$ and hence may allow directed photothermal destruction of cells ${ }^{76}$ to which these NPs were targeted. This is referred to as photothermal therapy (PTT). Third, AuNPs ${ }^{202,203}$, CNTs ${ }^{201}$, and SNPs ${ }^{123}$ may serve as scaffold for targeted gene therapy or, in the case of SNPs, may be filled with chemotherapeutic drugs ${ }^{204}$ to attenuate the progression of atherosclerotic lesions.

Research committed to finding the most suitable target, as well as the accompanying ligand for the assessment of vulnerable atherosclerotic plaques, has increased tremendously in recent years. It should be mentioned that the molecular targets used in molecular imaging and therapy of tumors show great resemblance to the targets expressed in atherosclerotic plaques, including inflammation ${ }^{205}$, proteolytic activity ${ }^{206,207}$, neovascularization ${ }^{208,209}$, and apoptosis ${ }^{210,211}$. Although both pathologies have different origins and progressions, the shared molecular markers may aid in the development and testing of ligands for specific targeting of NPs towards vulnerable plaques, as research committed to targeted tumor treatment is more advanced than targeted atherosclerotic plaque therapy.

In conclusion, current optical techniques and associated nanoparticles are promising for their application in high-resolution and high-sensitivity stand-alone imaging platforms as well as a complementary technique to clinically accepted imaging platforms.

\section{Acknowledgement}

This work was supported by SenterNovem, BSIK 03033. 


\section{References}

1 S. A. Wickline, A. M. Neubauer, P. M. Winter, S. D. Caruthers and G. M. Lanza; "Molecular imaging and therapy of atherosclerosis with targeted nanoparticles", J Magn Reson Imaging 2007, 25(4) 667-80.

S. D. Caruthers, S. A. Wickline and G. M. Lanza; "Nanotechnological applications in medicine", Curr Opin Biotechnol 2007,

R. H. Muller and C. M. Keck; "Challenges and solutions for the delivery of biotech drugs--a review of drug nanocrystal technology and lipid nanoparticles", J Biotechnol 2004, 113(1-3) 151-70.

M. Ozkan; "Quantum dots and other nanoparticles: what can they offer to drug discovery?" Drug Discov Today 2004, 9(24) 1065-71.

V. Wagner, A. Dullaart, A. K. Bock and A. Zweck; "The emerging nanomedicine landscape", Nat Biotechnol 2006, 24(10) 1211-7.

W. Cai and X. Chen; "Nanoplatforms for targeted molecular imaging in living subjects", Small 2007, 3(11) 1840-54.

R. R. Anderson and J. A. Parrish; "The optics of human skin", J Invest Dermatol 1981, 77(1) 139.

J. C. Jung and M. J. Schnitzer; "Multiphoton endoscopy", Opt Lett 2003, 28(11) 902-4.

K. Konig, A. Ehlers, I. Riemann, S. Schenkl, R. Buckle and M. Kaatz; "Clinical two-photon microendoscopy", Microsc Res Tech 2007, 70(5) 398-402.

H. P. Buschman, E. T. Marple, M. L. Wach, B. Bennett, T. C. Schut, H. A. Bruining, A. V. Bruschke, A. van der Laarse and G. J. Puppels; "In vivo determination of the molecular composition of artery wall by intravascular Raman spectroscopy", Anal Chem 2000, 72(16) 3771-5.

J. G. Fujimoto; "Optical coherence tomography for ultrahigh resolution in vivo imaging", Nat Biotechnol 2003, 21(11) 1361-7. Fujimoto; "In vivo endoscopic optical biopsy with optical coherence tomography", Science 1997, 276(5321) 2037-9.

S. Sethuraman, S. R. Aglyamov, J. H. Amirian, R. W. Smalling and S. Y. Emelianov; "Intravascular photoacoustic imaging using an IVUS imaging catheter", IEEE Trans Ultrason Ferroelectr Freq Control 2007, 54(5) 978-86. 
W. Denk, J. H. Strickler and W. W. Webb; "Two-photon laser scanning fluorescence microscopy", Science 1990, 248(4951) 73-6.

A. Buehler, M. A. M. J. van Zandvoort, B. J. Stelt, T. M. Hackeng, B. H. G. J. Schrans-Stassen, A. Bennaghmouch, L. Hofstra, J. P. M. Cleutjens, A. Duijvestijn, M. B. Smeets, D. P. V. de Kleijn, M. J. Post and E. D. de Muinck; "cNGR: A Novel Homing Sequence for CD13/APN Targeted Molecular Imaging of Murine Cardiac Angiogenesis In vivo", Arterioscler Thromb Vasc Biol 2006, 26(12) 2681-2687.

R. T. Megens, M. G. Oude Egbrink, J. P. Cleutjens, M. J. Kuijpers, P. H. Schiffers, M. Merkx, D. W. Slaaf and M. A. van Zandvoort; "Imaging Collagen in Intact Viable Healthy and Atherosclerotic Arteries Using Fluorescently Labeled CNA35 and Two-Photon Laser Scanning Microscopy", Mol Imaging 2007, 6(4) 247-60.

A. Zoumi, X. Lu, G. S. Kassab and B. J. Tromberg; "Imaging coronary artery microstructure using second-harmonic and two-photon fluorescence microscopy", Biophys J 2004, 87(4) 2778-86.

T. J. Romer, J. F. Brennan, 3rd, T. C. Schut, R. Wolthuis, R. C. van den Hoogen, J. J. Emeis, A. van der Laarse, A. V. Bruschke and G. J. Puppels; "Raman spectroscopy for quantifying cholesterol in intact coronary artery wall", Atherosclerosis 1998, 141(1) 117-24.

H. P. Buschman, G. Deinum, J. T. Motz, M. Fitzmaurice, J. R. Kramer, A. van der Laarse, A. V. Bruschke and M. S. Feld; "Raman microspectroscopy of human coronary atherosclerosis: biochemical assessment of cellular and extracellular morphologic structures in situ", Cardiovasc Pathol 2001, 10(2) 69-82. H. P. Buschman, J. T. Motz, G. Deinum, T. J. Romer, M. Fitzmaurice, J. R. Kramer, A. van der Laarse, A. V. Bruschke and M. S. Feld; "Diagnosis of human coronary atherosclerosis by morphology-based Raman spectroscopy", Cardiovasc Pathol 2001, 10(2) 59-68.

S. Keren, C. Zavaleta, Z. Cheng, A. de la Zerda, O. Gheysens and S. S. Gambhir; "Noninvasive molecular imaging of small living subjects using Raman spectroscopy", Proc Natl Acad Sci U S A 2008, 105(15) 5844-9.

M. E. Brezinski, G. J. Tearney, B. E. Bouma, S. A. Boppart, M. R. Hee, E. A. Swanson, J. F. Southern and J. G. Fujimoto; "Imaging of coronary artery microstructure (in vitro) with optical coherence tomography", Am J Cardiol 1996, 77(1) 92-3. and J. G. Fujimoto; "Optical coherence tomography for optical biopsy. Properties and demonstration of vascular pathology", Circulation 1996, 93(6) 1206-13. 


\section{Chapter 3}

H. Yabushita, B. E. Bouma, S. L. Houser, H. T. Aretz, I. K. Jang, K. H. Schlendorf, C. R. Kauffman, M. Shishkov, D. H. Kang, E. F. Halpern and G. J. Tearney; "Characterization of human atherosclerosis by optical coherence tomography", Circulation 2002, 106(13) 1640-5.

M. Cilingiroglu, J. H. Oh, B. Sugunan, N. J. Kemp, J. Kim, S. Lee, H. N. Zaatari, D. Escobedo, S. Thomsen, T. E. Milner and M. D. Feldman; "Detection of vulnerable plaque in a murine model of atherosclerosis with optical coherence tomography", Catheter Cardiovasc Interv 2006, 67(6) 915-23.

I. K. Jang, B. E. Bouma, D. H. Kang, S. J. Park, S. W. Park, K. B. Seung, K. B. Choi, M. Shishkov, K. Schlendorf, E. Pomerantsev, S. L. Houser, H. T. Aretz and G. J. Tearney; "Visualization of coronary atherosclerotic plaques in patients using optical coherence tomography: comparison with intravascular ultrasound", J Am Coll Cardiol 2002, 39(4) 604-9.

B. D. MacNeill, I. K. Jang, B. E. Bouma, N. Iftimia, M. Takano, H. Yabushita, M. Shishkov, C. R. Kauffman, S. L. Houser, H. T. Aretz, D. DeJoseph, E. F. Halpern and G. J. Tearney; "Focal and multi-focal plaque macrophage distributions in patients with acute and stable presentations of coronary artery disease", J Am Coll Cardiol 2004, 44(5) 972-9.

Y. Wang, X. Xie, X. Wang, G. Ku, K. L. Gill, D. P. O'Neal, G. Stoica and L. V. Wang; "Photoacoustic tomography of a nanoshell contrast agent in the in vivo rat brain", Nano Lett 2004, 4(9) 1689-1692.

X. Yang, S. E. Skrabalak, Z. Y. Li, Y. Xia and L. V. Wang; "Photoacoustic tomography of a rat cerebral cortex in vivo with au nanocages as an optical contrast agent", Nano Lett 2007, 7(12) 3798-802.

X. Wang, Y. Pang, G. Ku, X. Xie, G. Stoica and L. V. Wang; "Noninvasive laser-induced photoacoustic tomography for structural and functional in vivo imaging of the brain", Nat Biotechnol 2003, 21(7) 803-6. the microvasculature with a high-frequency ultrasound array transducer", J Biomed Opt 2007, 12(1) 010501.

S. Sethuraman, J. H. Amirian, S. H. Litovsky, R. W. Smalling and S. Y. Emelianov; "Ex vivo characterization of atherosclerosis using intravascular photoacoustic imaging", Opt Express 2007, 15(25) 16657-16666.

S. Sethuraman, J. H. Amirian, S. H. Litovsky, R. W. Smalling and S. Y. Emelianov; "Spectroscopic intravascular photoacoustic imaging to differentiate atherosclerotic plaques", Opt Express 2008, 16(5) 3363-3367. 
G. Oberdorster, E. Oberdorster and J. Oberdorster; "Nanotoxicology: an emerging discipline evolving from studies of ultrafine particles", Environ Health Perspect 2005, 113(7) 823-39.

C. Kirchner, T. Liedl, S. Kudera, T. Pellegrino, A. Munoz Javier, H. E. Gaub, S. Stolzle, N. Fertig and W. J. Parak; "Cytotoxicity of colloidal CdSe and CdSe/ZnS nanoparticles", Nano Lett 2005, $5(2) 331-8$.

A. Hartschuh, H. N. Pedrosa, L. Novotny and T. D. Krauss; "Simultaneous fluorescence and Raman scattering from single carbon nanotubes", Science 2003, 301(5638) 1354-6.

C. M. Niemeyer; "Nanoparticles, Proteins, and Nucleic Acids: Biotechnolohy meets Materials Science", Angewandte Chemie International Edition 2001, 40(22) 4128-4158.

M. Bruchez, Jr., M. Moronne, P. Gin, S. Weiss and A. P. Alivisatos; "Semiconductor nanocrystals as fluorescent biological labels", Science 1998, 281(5385) 2013-6.

W. C. W. Chan and S. Nie; "Quantum Dot Bioconjugates for Ultrasensitive Nonisotopic Detection", Science 1998, 281(5385) 2016-2018.

W. C. Chan, D. J. Maxwell, X. Gao, R. E. Bailey, M. Han and S. Nie; "Luminescent quantum dots for multiplexed biological detection and imaging", Curr Opin Biotechnol 2002, 13(1) 40-6.

J. K. Jaiswal, H. Mattoussi, J. M. Mauro and S. M. Simon; "Long-term multiple color imaging of live cells using quantum dot bioconjugates", Nat Biotechnol 2003, 21(1) 47-51. stable core-shell fluorescent silica nanoparticles", Nano Lett 2005, 5(1) 113-7.

A. P. Alivisatos; "Semiconductor Clusters, Nanocrystals, and Quantum Dots", Science 1996, 271(5251) 933-937.

A. Eychmuller, A. Mews and H. Weller; "A quantum dot quantum well: $\mathrm{CdS} / \mathrm{HgS} / \mathrm{CdS}$ ", Chemical Physics Letters 1993, 208(1-2) 59-62.

C. B. Murray, D. J. Norris and M. G. Bawendi; "Synthesis and characterization of nearly monodisperse CdE (E = sulfur, selenium, tellurium) semiconductor nanocrystallites", J. Am. Chem. Soc. 1993, 115(19) 8706-8715.

M. A. Hines and P. Guyot-Sionnest; "Synthesis and Characterization of Strongly Luminescing ZnS-Capped CdSe Nanocrystals", J. Phys. Chem. 1996, 100(2) 468-471. Luminescent CdSe/CdS Core/Shell Nanocrystals with Photostability and Electronic Accessibility", J. Am. Chem. Soc. 1997, 119(30) 7019-7029. 


\section{Chapter 3}

A. M. Derfus, W. C. W. Chan and S. N. Bhatia; "Probing the Cytotoxicity of Semiconductor Quantum Dots", Nano Lett. 2004, 4(1) 11-18.

H. Soo Choi, W. Liu, P. Misra, E. Tanaka, J. P. Zimmer, B. Itty Ipe, M. G. Bawendi and J. V. Frangioni; "Renal clearance of quantum dots", Nat Biotech 2007, 25(10) 1165-1170.

R. S. Yang, L. W. Chang, J. P. Wu, M. H. Tsai, H. J. Wang, Y. C. Kuo, T. K. Yeh, C. S. Yang and P. Lin; "Persistent tissue kinetics and redistribution of nanoparticles, quantum dot 705, in mice: ICP-MS quantitative assessment." Environ Health Perspect 2007, 115(9) 1339-43.

H. C. Fischer, L. Liu, K. S. Pang and W. C. W. Chan; "Pharmacokinetics of Nanoscale Quantum Dots: In vivo Distribution, Sequestration, and Clearance in the Rat", Advanced Functional Materials 2006, 16(10) 1299-1305.

2 M. Omura, A. Tanaka, M. Hirata, M. Zhao, Y. Makita, N. Inoue, K. Gotoh and N. Ishinishi; " Testicular Toxicity of Gallium Arsenide, Indium Arsenide, and Arsenic Oxide in Rats by Repetitive Intratracheal Instillation", Fundamental and Applied Toxicology 1996, 32(1) 72-78.

J. P. Zimmer, S. W. Kim, S. Ohnishi, E. Tanaka, J. V. Frangioni and M. G. Bawendi; "Size Series of Small Indium Arsenide-Zinc Selenide Core-Shell Nanocrystals and Their Application to In vivo Imaging", J. Am. Chem. Soc. 2006, 128(8) 2526-2527.

Z. Zhelev, H. Ohba and R. Bakalova; "Single quantum dot-micelles coated with silica shell as potentially non-cytotoxic fluorescent cell tracers", J Am Chem Soc 2006, 128(19) 6324-5.

W. Cai, A. Hsu, Z.-B. Li and X. Chen; "Are quantum dots ready for in vivo imaging in human subjects?" Nanoscale Research Letters 2007, 2(6) 265-281.

W. Cai, D. W. Shin, K. Chen, O. Gheysens, Q. Cao, S. X. Wang, S. S. Gambhir and X. Chen; "Peptide-Labeled Near-Infrared Quantum Dots for Imaging Tumor Vasculature in Living Subjects", Nano Lett 2006, 6(4) 669-676.

X. Gao, Y. Cui, R. M. Levenson, L. W. Chung and S. Nie; "In vivo cancer targeting and imaging with semiconductor quantum dots", Nat Biotechnol 2004, 22(8) 969-76.

A. Cambi, D. S. Lidke, D. J. Arndt-Jovin, C. G. Figdor and T. M. Jovin; "Ligand-conjugated quantum dots monitor antigen uptake and processing by dendritic cells", Nano Lett 2007, 7(4) 9707.

B. Ballou, L. A. Ernst, S. Andreko, T. Harper, J. A. Fitzpatrick, A. S. Waggoner and M. P. Bruchez; "Sentinel lymph node imaging using quantum dots in mouse tumor models", Bioconjug Chem 2007, 18(2) 389-96.

S. Kim, Y. T. Lim, E. G. Soltesz, A. M. De Grand, J. Lee, A. Nakayama, J. A. Parker, T. Mihaljevic, R. G. Laurence, D. M. Dor, L. H. Cohn, M. G. Bawendi and J. V. Frangioni; "Near-infrared fluo- 
rescent type II quantum dots for sentinel lymph node mapping", Nat Biotechnol 2004, 22(1) 93-7.

X. Wu, H. Liu, J. Liu, K. N. Haley, J. A. Treadway, J. P. Larson, N. Ge, F. Peale and M. P. Bruchez; "Immunofluorescent labeling of cancer marker Her2 and other cellular targets with semiconductor quantum dots", Nat Biotech 2003, 21(1) 41-46.

E. Kahn, A. Vejux, F. Menetrier, C. Maiza, A. Hammann, A. Sequeira-Le Grand, F. Frouin, Y. Tourneur, F. Brau, J. M. Riedinger, E. Steinmetz, A. Todd-Pokropek and G. Lizard; "Analysis of CD36 expression on human monocytic cells and atherosclerotic tissue sections with quantum dots: investigation by flow cytometry and spectral imaging microscopy", Anal Quant Cytol Histol 2006, 28(1) 14-26. namic shear stresses in mouse aortas: implications for atherogenesis", Arterioscler Thromb Vasc Biol 2007, 27(2) 346-51.

L. Prinzen, R. J. Miserus, A. Dirksen, T. M. Hackeng, N. Deckers, N. J. Bitsch, R. T. Megens, K. Douma, J. W. Heemskerk, M. E. Kooi, P. M. Frederik, D. W. Slaaf, M. A. van Zandvoort and C. P. Reutelingsperger; "Optical and magnetic resonance imaging of cell death and platelet activation using annexin a5-functionalized quantum dots", Nano Lett 2007, 7(1) 93-100. A. Jayagopal, P. K. Russ and F. R. Haselton; "Surface engineering of quantum dots for in vivo vascular imaging", Bioconjug Chem 2007, 18(5) 1424-33. H. E. Grecco, K. A. Lidke, R. Heintzmann, D. S. Lidke, C. Spagnuolo, O. E. Martinez, E. A. JaresErijman and T. M. Jovin; "Ensemble and single particle photophysical properties (two-photon excitation, anisotropy, FRET, lifetime, spectral conversion) of commercial quantum dots in solution and in live cells", Microsc Res Tech 2004, 65(4-5) 169-79.

R. Weissleder, C. H. Tung, U. Mahmood and A. Bogdanov, Jr.; "In vivo imaging of tumors with protease-activated near-infrared fluorescent probes", Nat Biotechnol 1999, 17(4) 375-8.

M. K. So, C. Xu, A. M. Loening, S. S. Gambhir and J. Rao; "Self-illuminating quantum dot conjugates for in vivo imaging", Nat Biotechnol 2006, K. Kampani, K. Quann, J. Ahuja, B. Wigdahl, Z. K. Khan and P. Jain; "A novel high throughput quantum dot-based fluorescence assay for quantitation of virus binding and attachment", Journal of Virological Methods 2007, 141(2) 125-132. electron microscopy", J Histochem Cytochem 1977, 25(4) 295-305. 


\section{Chapter 3}

71

J. Zheng, J. T. Petty and R. M. Dickson; "High quantum yield blue emission from water-soluble Au8 nanodots", J Am Chem Soc 2003, 125(26) 7780-1.

M. Montalti, N. Zaccheroni, L. Prodi, N. O'Reilly and S. L. James; "Enhanced sensitized NIR luminescence from gold nanoparticles via energy transfer from surface-bound fluorophores", J Am Chem Soc 2007, 129(9) 2418-9.

3 M. M. Alvarez, J. T. Khoury, T. G. Schaaff, M. N. Shafigullin, I. Vezmar and R. L. Whetten; "Optical Absorption Spectra of Nanocrystal Gold Molecules", Journal of Physical Chemistry B 1997, 101(3706-3712.

R. A. Farrer, F. L. Butterfield, V. W. Chen and J. T. Fourkas; "Highly efficient multiphotonabsorption-induced luminescence from gold nanoparticles", Nano Lett 2005, 5(6) 1139-42.

5

J. Chen, F. Saeki, B. J. Wiley, H. Cang, M. J. Cobb, Z. Y. Li, L. Au, H. Zhang, M. B. Kimmey, X. Li and Y. Xia; "Gold nanocages: bioconjugation and their potential use as optical imaging contrast agents", Nano Lett 2005, 5(3) 473-7.

6

X. Huang, I. H. El-Sayed, W. Qian and M. A. El-Sayed; "Cancer cell imaging and photothermal therapy in the near-infrared region by using gold nanorods", J Am Chem Soc 2006, 128(6) 2115-20.

7 R. Shukla, V. Bansal, M. Chaudhary, A. Basu, R. R. Bhonde and M. Sastry; "Biocompatibility of gold nanoparticles and their endocytotic fate inside the cellular compartment: a microscopic overview", Langmuir 2005, 21(23) 10644-54.

W. B. Tan and Y. Zhang; "Surface modification of gold and quantum dot nanoparticles with chitosan for bioapplications", J Biomed Mater Res A 2005, 75(1) 56-62.

D. Kim, S. Park, J. H. Lee, Y. Y. Jeong and S. Jon; "Antibiofouling polymer-coated gold nanoparticles as a contrast agent for in vivo X-ray computed tomography imaging", J Am Chem Soc 2007, 129(24) 7661-5.

P. Mukherjee, R. Bhattacharya, P. Wang, L. Wang, S. Basu, J. A. Nagy, A. Atala, D. Mukhopadhyay and S. Soker; "Antiangiogenic properties of gold nanoparticles", Clin Cancer Res 2005, 11(9) 3530-4.

1 G. Renaud, R. L. Hamilton and R. J. Havel; "Hepatic metabolism of colloidal gold-low-density lipoprotein complexes in the rat: evidence for bulk excretion of lysosomal contents into bile", Hepatology 1989, 9(3) 380-92.

2 R. Rau, G. Herborn, H. Menninger and O. Sangha; "Radiographic outcome after three years of patients with early erosive rheumatoid arthritis treated with intramuscular methotrexate or 
parenteral gold. Extension of a one-year double-blind study in 174 patients", Rheumatology (Oxford) 2002, 41(2) 196-204.

F. Wolfe and D. J. Hawley; "The relationship between clinical activity and depression in rheumatoid arthritis", J Rheumatol 1993, 20(12) 2032-7.

T. K. Kvien, H. K. Zeidler, P. Hannonen, F. A. Wollheim, O. Forre, I. Hafstrom, J. P. Kaltwasser, M. Leirisalo-Repo, B. Manger, L. Laasonen, H. Prestele and P. Kurki; "Long term efficacy and safety of cyclosporin versus parenteral gold in early rheumatoid arthritis: a three year study of radiographic progression, renal function, and arterial hypertension", Ann Rheum Dis 2002, 61(6) 511-6.

A. Hafejee and M. J. Burke; "Acute pneumonitis starting 2 hours after intramuscular gold administration in a patient with rheumatoid arthritis", Ann Rheum Dis 2004, 63(11) 1525-6. K. Hiramatsu, H. Ishizuka and M. Yamasaki; "Bronchiolar disease associated with gold compounds administration in a patient with rheumatoid arthritis", Mod Rheumatol 2005, 15(2) $148-52$.

D. V. Leff, L. Brandt and J. R. Heath; "Synthesis and Characterization of Hydrophobic, Organically-Soluble Gold Nanocrystals Functionalized with Primary Amines", Langmuir 1996, 12(20) 4723-4730.

T. Huang and R. W. Murray; "Visible Luminescence of Water-Soluble Monolayer-Protected Gold Clusters", J. Phys. Chem. B 2001, 105(50) 12498-12502.

P. R. Selvakannan, S. Mandal, S. Phadtare, R. Pasricha and M. Sastry; "Capping of Gold Nanoparticles by the Amino Acid Lysine Renders Them Water-Dispersible", Langmuir 2003, 19(8) 3545-3549.

A. Gole, C. Dash, C. Soman, S. R. Sainkar, M. Rao and M. Sastry; "On the preparation, characterization, and enzymatic activity of fungal protease-gold colloid bioconjugates", Bioconjug Chem 2001, 12(5) 684-90. P. Selvakannan, S. Mandal, S. Phadtare, A. Gole, R. Pasricha, S. D. Adyanthaya and M. Sastry; "Water-dispersible tryptophan-protected gold nanoparticles prepared by the spontaneous reduction of aqueous chloroaurate ions by the amino acid", J Colloid Interface Sci 2004, 269(1) 97-102.

K. Sokolov, M. Follen, J. Aaron, I. Pavlova, A. Malpica, R. Lotan and R. Richards-Kortum; "Realtime vital optical imaging of precancer using anti-epidermal growth factor receptor antibodies conjugated to gold nanoparticles", Cancer Res 2003, 63(9) 1999-2004. 


\section{Chapter 3}

H. Wang, T. B. Huff, D. A. Zweifel, W. He, P. S. Low, A. Wei and J. X. Cheng; "In vitro and in vivo two-photon luminescence imaging of single gold nanorods", Proc Natl Acad Sci U S A 2005, 102(44) 15752-6.

N. J. Durr, T. Larson, D. K. Smith, B. A. Korgel, K. Sokolov and A. Ben-Yakar; "Two-Photon Luminescence Imaging of Cancer Cells Using Molecularly Targeted Gold Nanorods", Nano Lett. 2007, 7(4) 941-945.

Y. Colpin, A. Swan, A. V. Zvyagin and T. Plakhotnik; "Imaging and sizing of diamond nanoparticles", Opt Lett 2006, 31(5) 625-7.

C. C. Fu, H. Y. Lee, K. Chen, T. S. Lim, H. Y. Wu, P. K. Lin, P. K. Wei, P. H. Tsao, H. C. Chang and W. Fann; "Characterization and application of single fluorescent nanodiamonds as cellular biomarkers", Proc Natl Acad Sci U S A 2007, 104(3) 727-32.

S. J. Yu, M. W. Kang, H. C. Chang, K. M. Chen and Y. C. Yu; "Bright fluorescent nanodiamonds: no photobleaching and low cytotoxicity", J Am Chem Soc 2005, 127(50) 17604-5.

A. Beveratos, R. Brouri, T. Gacoin, J. P. Poizat and P. Grangier; "Nonclassical radiation from diamond nanocrystals", Physical Review A 2001, 64(6)

R. Brouri, A. Beveratos, J. P. Poizat and P. Grangier; "Photon antibunching in the fluorescence of individual color centers in diamond", Opt. Lett. 2000, Opt. Lett.(17) 1294-6.

T. L. Wee, Y. K. Tzeng, C. C. Han, H. C. Chang, W. Fann, J. H. Hsu, K. M. Chen and Y. C. Yu; "Two-photon excited fluorescence of nitrogen-vacancy centers in proton-irradiated type Ib diamond", J Phys Chem A 2007, 111(38) 9379-86.

101

C. Y. Cheng, E. Perevedentseva, J. S. Tu, P. H. Chung, C. L. Cheng, K. K. Liu, J. I. Chao, P. H. Chen and C. C. Chang; "Direct and in vitro observation of growth hormone receptor molecules in A549 human lung epithelial cells by nanodiamond labeling", Applied Physics Letters 2007, 90(16) 163903-3.

102 A. Kruger, F. Kataoka, M. Ozawa, T. Fujino, Y. Suzuki, A. E. Aleksenskii, A. Y. Vul and E. Osawa; "Unusually tight aggregation in detonation nanodiamond: Identification and disintegration", Carbon 2005, 43(8) 1722-1730.

103 K. Bakowizc and S. Mitura; "Biocompatibility of NCD", Journal of Wide Bandgap Materials 2002, $9(261-272$. Murray, S. Y. Xie and Y. P. Sun; "Carbon dots for multiphoton bioimaging", J Am Chem Soc 2007, 129(37) 11318-9. 

Wang, H. Wang, P. G. Luo, H. Yang, M. E. Kose, B. Chen, L. M. Veca and S. Y. Xie; "Quantumsized carbon dots for bright and colorful photoluminescence", J Am Chem Soc 2006, 128(24) 7756-7.

T. K. Leeuw, R. M. Reith, R. A. Simonette, M. E. Harden, P. Cherukuri, D. A. Tsyboulski, K. M. Beckingham and R. B. Weisman; "Single-walled carbon nanotubes in the intact organism: near-IR imaging and biocompatibility studies in Drosophila", Nano Lett 2007, 7(9) 2650-4. D. A. Heller, R. M. Mayrhofer, S. Baik, Y. V. Grinkova, M. L. Usrey and M. S. Strano; "Concomitant length and diameter separation of single-walled carbon nanotubes", J Am Chem Soc 2004, 126(44) 14567-73.

J. Lefebvre, D. G. Austing, J. Bond and P. Finnie; "Photoluminescence Imaging of Suspended Single-Walled Carbon Nanotubes", Nano Lett. 2006, 6(8) 1603-1608.

H. Dai; "Noncovalent functionalization of carbon nanotubes for highly specific electronic biosensors", Proc Natl Acad Sci U S A 2003, 100(9) 4984-9.

S. Campidelli, C. Sooambar, E. Lozano Diz, C. Ehli, D. M. Guldi and M. Prato; "Dendrimerfunctionalized single-wall carbon nanotubes: synthesis, characterization, and photoinduced electron transfer", J Am Chem Soc 2006, 128(38) 12544-52.

Y. Wu, J. S. Hudson, Q. Lu, J. M. Moore, A. S. Mount, A. M. Rao, E. Alexov and P. C. Ke; "Coating Single-Walled Carbon Nanotubes with Phospholipids", J. Phys. Chem. B 2006, 110(6) 24752478.

112 N. W. S. Kam, T. C. Jessop, P. Wender and H. Dai; "Nanotube Molecular Transporters: Internalization of Carbon Nanotube-Protein Conjugates into Mammalian Cells", Journal of the American Society 2004, 126(6850-6851.

113 K. Pulskamp, S. Diabate and H. F. Krug; "Carbon nanotubes show no sign of acute toxicity but induce intracellular reactive oxygen species in dependence on contaminants", Toxicol Lett 2007, 168(1) 58-74.

114 P. Cherukuri, S. M. Bachilo, S. H. Litovsky and R. B. Weisman; "Near-infrared fluorescence microscopy of single-walled carbon nanotubes in phagocytic cells", J Am Chem Soc 2004, 126(48) 15638-9.

115 N. A. Monteiro-Riviere, R. J. Nemanich, A. O. Inman, Y. Y. Wang and J. E. Riviere; "Multiwalled carbon nanotube interactions with human epidermal keratinocytes", Toxicology Letters 2005, 155(3) 377-384. 


\section{Chapter 3}

116 L. Zhu, D. W. Chang, L. Dai and Y. Hong; "DNA damage induced by multiwalled carbon nanotubes in mouse embryonic stem cells", Nano Lett 2007, 7(12) 3592-7.

117 P. Cherukuri, C. J. Gannon, T. K. Leeuw, H. K. Schmidt, R. E. Smalley, S. A. Curley and R. B. Weisman; "Mammalian pharmacokinetics of carbon nanotubes using intrinsic near-infrared fluorescence", Proc Natl Acad Sci U S A 2006, 103(50) 18882-6.

118 R. Singh, D. Pantarotto, L. Lacerda, G. Pastorin, C. Klumpp, M. Prato, A. Bianco and K. Kostarelos; "Tissue biodistribution and blood clearance rates of intravenously administered carbon nanotube radiotracers", Proceedings of the National Academy of Sciences 2006, 103(9) 33573362.

119 N. W. Kam, M. O'Connell, J. A. Wisdom and H. Dai; "Carbon nanotubes as multifunctional biological transporters and near-infrared agents for selective cancer cell destruction", Proc Natl Acad Sci U S A 2005, 102(33) 11600-5.

120 X. Zhao, R. P. Bagwe and W. Tan; "Development of Organic-Dye-Doped Silica Nanoparticles in a Reverse Microemulsion", Adv Mater 2004, 16(2) 173-6.

121 M. Montalti, L. Prodi, N. Zaccheroni, A. Zattoni, P. Reschiglian and G. Falini; "Energy transfer in fluorescent silica nanoparticles", Langmuir 2004, 20(7) 2989-91.

R. Mallik, C. Wa and D. S. Hage; "Development of sulfhydryl-reactive silica for protein immobilization in high-performance affinity chromatography", Anal Chem 2007, 79(4) 1411-24.

I. Roy, T. Y. Ohulchanskyy, D. J. Bharali, H. E. Pudavar, R. A. Mistretta, N. Kaur and P. N. Prasad; "Optical tracking of organically modified silica nanoparticles as DNA carriers: a nonviral, nanomedicine approach for gene delivery", Proc Natl Acad Sci U S A 2005, 102(2) 279-84.

124 X. Xing, X. He, J. Peng, K. Wang and W. Tan; "Uptake of silica-coated nanoparticles by HeLa cells", J Nanosci Nanotechnol 2005, 5(10) 1688-93.

J. S. Chang, K. L. Chang, D. F. Hwang and Z. L. Kong; "In vitro cytotoxicitiy of silica nanoparticles at high concentrations strongly depends on the metabolic activity type of the cell line", Environ Sci Technol 2007, 41(6) 2064-8.

Y. Jin, S. Kannan, M. Wu and J. X. Zhao; "Toxicity of luminescent silica nanoparticles to living cells", Chem Res Toxicol 2007, 20(8) 1126-33.

127 K. Peters, R. E. Unger, C. J. Kirkpatrick, A. M. Gatti and E. Monari; "Effects of nano-scaled particles on endothelial cell function in vitro: studies on viability, proliferation and inflammation", J Mater Sci Mater Med 2004, 15(4) 321-5. 
J. Choi, A. A. Burns, R. M. Williams, Z. Zhou, A. Flesken-Nikitin, W. R. Zipfel, U. Wiesner and A. Y. Nikitin; "Core-shell silica nanoparticles as fluorescent labels for nanomedicine", J Biomed Opt 2007, 12(6) 064007.

S. Santra, B. Liesenfeld, D. Dutta, D. Chatel, C. D. Batich, W. Tan, B. M. Moudgil and R. A. Mericle; "Folate conjugated fluorescent silica nanoparticles for labeling neoplastic cells", J Nanosci Nanotechnol 2005, 5(6) 899-904.

L. Wang, W. Zhao, M. B. O'Donoghue and W. Tan; "Fluorescent nanoparticles for multiplexed bacteria monitoring", Bioconjug Chem 2007, 18(2) 297-301.

B. O. Dabbousi, J. Rodriguez-Viejo, F. V. Mikulec, J. R. Heine, H. Mattoussi, R. Ober, K. F. Jensen and M. G. Bawendi; "(CdSe)ZnS Core-Shell Quantum Dots: Synthesis and Characterization of a Size Series of Highly Luminescent Nanocrystallites", J. Phys. Chem. B 1997, 101(46) 94639475.

M. J. O'Connell, S. M. Bachilo, C. B. Huffman, V. C. Moore, M. S. Strano, E. H. Haroz, K. L. Rialon, P. J. Boul, W. H. Noon, C. Kittrell, J. Ma, R. H. Hauge, R. B. Weisman and R. E. Smalley; "Band gap fluorescence from individual single-walled carbon nanotubes", Science 2002, 297(5581) 593-6.

H.-C. Chang and K. Chen; "Nanodiamond as a possible carrier for extended red emission", The Astrophysical journal 2006, 639(2) L63-L66.

F. Treaussart, V. Jacques, E. Wu, T. Gacoin, P. Grangier and J.-F. Roch; "Photoluminescence of single colour defects in 50nm diamond nanocrystals", Physica B 2006, 376-377(

35 C. Barbé, J. Bartlett, L. Kong, K. Finiie, H. Q. Lin, M. Larkin, S. Calleja, A. Bush and Calleja; "Silica particles: A Novel drug Delivery System", Advanced Materials 2004, 16(21) 1959-66.

H. C. Stary, A. B. Chandler, S. Glagov, J. R. Guyton, W. Insull, Jr., M. E. Rosenfeld, S. A. Schaffer, C. J. Schwartz, W. D. Wagner and R. W. Wissler; "A definition of initial, fatty streak, and intermediate lesions of atherosclerosis. A report from the Committee on Vascular Lesions of the Council on Arteriosclerosis, American Heart Association", Circulation 1994, 89(5) 246278.

H. C. Stary, A. B. Chandler, R. E. Dinsmore, V. Fuster, S. Glagov, W. Insull, Jr., M. E. Rosenfeld, C. J. Schwartz, W. D. Wagner and R. W. Wissler; "A Definition of Advanced Types of Atherosclerotic Lesions and a Histological Classification of Atherosclerosis : A Report From the Committee on Vascular Lesions of the Council on Arteriosclerosis, American Heart Association", Circulation 1995, 92(5) 1355-1374. 
J. Sanz and Z. A. Fayad; "Imaging of atherosclerotic cardiovascular disease", Nature 2008, 451(7181) 953-7.

M. Naghavi, P. Libby, E. Falk, S. W. Casscells, S. Litovsky, J. Rumberger, J. J. Badimon, C. Stefanadis, P. Moreno, G. Pasterkamp, Z. Fayad, P. H. Stone, S. Waxman, P. Raggi, M. Madjid, A. Zarrabi, A. Burke, C. Yuan, P. J. Fitzgerald, D. S. Siscovick, C. L. de Korte, M. Aikawa, K. E. Juhani Airaksinen, G. Assmann, C. R. Becker, J. H. Chesebro, A. Farb, Z. S. Galis, C. Jackson, I.-K. Jang, W. Koenig, R. A. Lodder, K. March, J. Demirovic, M. Navab, S. G. Priori, M. D. Rekhter, R. Bahr, S. M. Grundy, R. Mehran, A. Colombo, E. Boerwinkle, C. Ballantyne, W. Insull, Jr., R. S. Schwartz, R. Vogel, P. W. Serruys, G. K. Hansson, D. P. Faxon, S. Kaul, H. Drexler, P. Greenland, J. E. Muller, R. Virmani, P. M. Ridker, D. P. Zipes, P. K. Shah and J. T. Willerson; "From Vulnerable Plaque to Vulnerable Patient: A Call for New Definitions and Risk Assessment Strategies: Part I", Circulation 2003, 108(14) 1664-1672.

M. Oostendorp, K. Douma, T. M. Hackeng, A. Dirksen, M. J. Post, M. A. Van Zandvoort and W. H. Backes; "In vivo $T_{1}$ and $T_{2}$ effects of paramagnetic Quantum Dot based contrast agents for molecular Magnetic Resonance Imaging", Proc Intl Soc Mag Reson Med 2008, 16(1699. and J. Narula; "Noninvasive detection of plaque instability with use of radiolabeled annexin A5 in patients with carotid-artery atherosclerosis", N Engl J Med 2004, 350(14) 1472-3.

A. Broisat, L. M. Riou, V. Ardisson, D. Boturyn, P. Dumy, D. Fagret and C. Ghezzi; "Molecular imaging of vascular cell adhesion molecule-1 expression in experimental atherosclerotic plaques with radiolabelled B2702-p", Eur J Nucl Med Mol Imaging 2007, 34(6) 830-40. "In vivo imaging of activated endothelium using an anti-VCAM-1 magnetooptical probe", Bioconjug Chem 2005, 16(3) 576-81.

B. A. Kaufmann, J. M. Sanders, C. Davis, A. Xie, P. Aldred, I. J. Sarembock and J. R. Lindner; "Molecular imaging of inflammation in atherosclerosis with targeted ultrasound detection of vascular cell adhesion molecule-1", Circulation 2007, 116(3) 276-84.

M. A. McAteer, N. R. Sibson, C. von Zur Muhlen, J. E. Schneider, A. S. Lowe, N. Warrick, K. M. Channon, D. C. Anthony and R. P. Choudhury; "In vivo magnetic resonance imaging of acute brain inflammation using microparticles of iron oxide", Nat Med 2007, 13(10) 1253-8. tection of vascular adhesion molecule-1 expression using a novel multimodal nanoparticle", Circ Res 2005, 96(3) 327-36. 
M. Nahrendorf, F. A. Jaffer, K. A. Kelly, D. E. Sosnovik, E. Aikawa, P. Libby and R. Weissleder; "Noninvasive vascular cell adhesion molecule-1 imaging identifies inflammatory activation of cells in atherosclerosis", Circulation 2006, 114(14) 1504-11.

M. A. McAteer, J. E. Schneider, Z. A. Ali, N. Warrick, C. A. Bursill, C. von zur Muhlen, D. R. Greaves, S. Neubauer, K. M. Channon and R. P. Choudhury; "Magnetic resonance imaging of endothelial adhesion molecules in mouse atherosclerosis using dual-targeted microparticles of iron oxide", Arterioscler Thromb Vasc Biol 2008, 28(1) 77-83. J. J. Rychak, J. R. Lindner, K. Ley and A. L. Klibanov; "Deformable gas-filled microbubbles targeted to P-selectin", J Control Release 2006, 114(3) 288-99. P. Libby; "Inflammation in atherosclerosis: visualizing matrix metalloproteinase action in macrophages in vivo", Circulation 2006, 114(1) 55-62. F. A. Jaffer, D. E. Kim, L. Quinti, C. H. Tung, E. Aikawa, A. N. Pande, R. H. Kohler, G. P. Shi, P. Libby and R. Weissleder; "Optical visualization of cathepsin $K$ activity in atherosclerosis with a novel, protease-activatable fluorescence sensor", Circulation 2007, 115(17) 2292-8. V. Amirbekian, M. J. Lipinski, K. C. Briley-Saebo, S. Amirbekian, J. G. Aguinaldo, D. B. Weinreb, E. Vucic, J. C. Frias, F. Hyafil, V. Mani, E. A. Fisher and Z. A. Fayad; "Detecting and assessing macrophages in vivo to evaluate atherosclerosis noninvasively using molecular MRI", Proc Natl Acad Sci U S A 2007, 104(3) 961-6.

D. R. Elmaleh, A. J. Fischman, A. Tawakol, A. Zhu, T. M. Shoup, U. Hoffmann, A. L. Brownell and P. C. Zamecnik; "Detection of inflamed atherosclerotic lesions with diadenosine-5',5"'P1,P4-tetraphosphate (Ap4A) and positron-emission tomography", Proc Natl Acad Sci U S A 2006, 103(43) 15992-6.

154 A. Tawakol, R. Q. Migrino, G. G. Bashian, S. Bedri, D. Vermylen, R. C. Cury, D. Yates, G. M. LaMuraglia, K. Furie, S. Houser, H. Gewirtz, J. E. Muller, T. J. Brady and A. J. Fischman; "In vivo 18F-fluorodeoxyglucose positron emission tomography imaging provides a noninvasive measure of carotid plaque inflammation in patients", J Am Coll Cardiol 2006, 48(9) 1818-24.

Z. Zhang, J. Machac, G. Helft, S. G. Worthley, C. Tang, A. G. Zaman, O. J. Rodriguez, M. S. Buchsbaum, V. Fuster and J. J. Badimon; "Non-invasive imaging of atherosclerotic plaque macrophage in a rabbit model with F-18 FDG PET: a histopathological correlation", BMC Nucl Med 2006, 6(3. 
"Noninvasive imaging of atherosclerotic lesions in apolipoprotein E-deficient and low-densitylipoprotein receptor-deficient mice with annexin A5", J Nucl Med 2006, 47(9) 1497-505.

F. D. Kolodgie, A. Petrov, R. Virmani, N. Narula, J. W. Verjans, D. K. Weber, D. Hartung, N. Steinmetz, J. L. Vanderheyden, M. A. Vannan, H. K. Gold, C. P. Reutelingsperger, L. Hofstra and J. Narula; "Targeting of apoptotic macrophages and experimental atheroma with radiolabeled annexin V: a technique with potential for noninvasive imaging of vulnerable plaque", Circulation 2003, 108(25) 3134-9.

B. R. Smith, J. Heverhagen, M. Knopp, P. Schmalbrock, J. Shapiro, M. Shiomi, N. I. Moldovan, M. Ferrari and S. C. Lee; "Localization to atherosclerotic plaque and biodistribution of biochemically derivatized superparamagnetic iron oxide nanoparticles (SPIONs) contrast particles for magnetic resonance imaging (MRI)", Biomed Microdevices 2007, 9(5) 719-27.

P. M. Winter, A. M. Morawski, S. D. Caruthers, R. W. Fuhrhop, H. Zhang, T. A. Williams, J. S. Allen, E. K. Lacy, J. D. Robertson, G. M. Lanza and S. A. Wickline; "Molecular imaging of angiogenesis in early-stage atherosclerosis with alpha(v)beta3-integrin-targeted nanoparticles", Circulation 2003, 108(18) 2270-4.

H. Wang, W. Cai, K. Chen, Z. B. Li, A. Kashefi, L. He and X. Chen; "A new PET tracer specific for vascular endothelial growth factor receptor 2", Eur J Nucl Med Mol Imaging 2007,

M. V. Backer, Z. Levashova, V. Patel, B. T. Jehning, K. Claffey, F. G. Blankenberg and J. M. Backer; "Molecular imaging of VEGF receptors in angiogenic vasculature with single-chain VEGF-based probes", Nat Med 2007, 13(4) 504-9.

C. v. Bary, A. Preissel, E. Spuentrup, A. Keithahn, S. Schachoff, S. Robinson, J. Lazewatsky, M. Schwaiger, J. Hausleiter and R. M. Botnar; "Molecular MRI of vascular remodeling in a swine model of coronary injury using an elastin-binding contrast agent", Proc Intl Soc Mag Reson Med 2008, 16(321.

R. M. Botnar, A. S. Perez, S. Witte, A. J. Wiethoff, J. Laredo, J. Hamilton, W. Quist, E. C. Parsons, Jr., A. Vaidya, A. Kolodziej, J. A. Barrett, P. B. Graham, R. M. Weisskoff, W. J. Manning and M. T. Johnstone; "In vivo molecular imaging of acute and subacute thrombosis using a fibrin-binding magnetic resonance imaging contrast agent", Circulation 2004, 109(16) 2023-9. E. Spuentrup, A. Buecker, M. Katoh, A. J. Wiethoff, E. C. Parsons, Jr., R. M. Botnar, R. M. Weisskoff, P. B. Graham, W. J. Manning and R. W. Gunther; "Molecular magnetic resonance imaging of coronary thrombosis and pulmonary emboli with a novel fibrin-targeted contrast agent", Circulation 2005, 111(11) 1377-82. 
Magnetic Resonance Imaging of Atrial Clots in a Swine Model", Circulation 2005, 112(3) 396399.

E. Spuentrup, M. Katoh, A. Buecker, B. Fausten, A. J. Wiethoff, J. E. Wildberger, P. Haage, E. C. Parsons, Jr., R. M. Botnar, P. B. Graham, M. Vettelschoss and R. W. Gunther; "Molecular MR imaging of human thrombi in a swine model of pulmonary embolism using a fibrin-specific contrast agent", Invest Radiol 2007, 42(8) 586-95.

167 C. P. Stracke, M. Katoh, A. J. Wiethoff, E. C. Parsons, P. Spangenberg and E. Spuntrup; "Molecular MRI of cerebral venous sinus thrombosis using a new fibrin-specific MR contrast agent", Stroke 2007, 38(5) 1476-81.

168 M. R. Aruva, J. Daviau, S. S. Sharma and M. L. Thakur; "Imaging thromboembolism with fibrinavid 99mTc-peptide: evaluation in swine", J Nucl Med 2006, 47(1) 155-62.

169 R. J. J. Miserus, L. Prinzen, M. V. Herias, T. M. Hackeng, A. Dirksen, W. Adriaens, M. A. M. J. Van Zandvoort, M. B. Lobbes, M. J. Daemen, J. J. Van Engelshoven, S. Heeneman and M. E. Kooi; "In vivo MR Imaging of Acute Thrombus Formation Using a Bimodal Contrast Agent", Proc Intl Soc Mag Reson Med 2008, $16(22$.

170 A. Alonso, A. Della Martina, M. Stroick, M. Fatar, M. Griebe, S. Pochon, M. Schneider, M. Hennerici, E. Allemann and S. Meairs; "Molecular imaging of human thrombus with novel abciximab immunobubbles and ultrasound", Stroke 2007, 38(5) 1508-14.

171 B. Wang, W. J. Zang, M. Wang, H. Ai, Y. W. Wang, Y. P. Li, G. S. He, L. Wang and X. J. Yu; "Prolonging the ultrasound signal enhancement from thrombi using targeted microbubbles based on sulfur-hexafluoride-filled gas", Acad Radiol 2006, 13(4) 428-33.

172 L. Sarda-Mantel, M. Coutard, F. Rouzet, O. Raguin, J. M. Vrigneaud, F. Hervatin, G. Martet, Z. Touat, P. Merlet, D. Le Guludec and J. B. Michel; "99mTc-annexin-V functional imaging of luminal thrombus activity in abdominal aortic aneurysms", Arterioscler Thromb Vasc Biol 2006, 26(9) 2153-9.

173 J. E. Adrian, J. A. Kamps, K. Poelstra, G. L. Scherphof, D. K. Meijer and Y. Kaneda; "Delivery of viral vectors to hepatic stellate cells in fibrotic livers using HVJ envelopes fused with targeted liposomes", J Drug Target 2007, 15(1) 75-82.

174 P. C. Rensen, J. C. Gras, E. K. Lindfors, K. W. van Dijk, J. W. Jukema, T. J. van Berkel and E. A. Biessen; "Selective targeting of liposomes to macrophages using a ligand with high affinity for the macrophage scavenger receptor class A", Curr Drug Discov Technol 2006, 3(2) 135-44.

175 W. C. Zamboni; "Concept and Clinical Evaluation of Carrier-Mediated Anticancer Agents", Oncologist 2008, 13(3) 248-260. 


\section{Chapter 3}

176 W. J. Mulder, K. Douma, G. A. Koning, M. A. van Zandvoort, E. Lutgens, M. J. Daemen, K. Nicolay and G. J. Strijkers; "Liposome-enhanced MRI of neointimal lesions in the ApoE-KO mouse", Magn Reson Med 2006, 55(5) 1170-4.

177 W. J. Mulder, G. J. Strijkers, A. W. Griffioen, L. van Bloois, G. Molema, G. Storm, G. A. Koning and K. Nicolay; "A liposomal system for contrast-enhanced magnetic resonance imaging of molecular targets", Bioconjug Chem 2004, 15(4) 799-806.

178 G. A. van Tilborg, W. J. Mulder, N. Deckers, G. Storm, C. P. Reutelingsperger, G. J. Strijkers and K. Nicolay; "Annexin A5-functionalized bimodal lipid-based contrast agents for the detection of apoptosis", Bioconjug Chem 2006, 17(3) 741-9.

179 W. J. M. Mulder, G. J. Strijkers, J. W. Habets, E. J. W. Bleeker, D. W. J. van der Schaft, G. Storm, G. A. Koning, A. W. Griffioen and K. Nicolay; "MR molecular imaging and fluorescence microscopy for identification of activated tumor endothelium using a bimodal lipidic nanoparticle", FASEB J. 2005, 05-4145fje.

180 W. J. Mulder, G. J. Strijkers, K. C. Briley-Saboe, J. C. Frias, J. G. Aguinaldo, E. Vucic, V. Amirbekian, C. Tang, P. T. Chin, K. Nicolay and Z. A. Fayad; "Molecular imaging of macrophages in atherosclerotic plaques using bimodal PEG-micelles", Magn Reson Med 2007, 58(6) 1164-70.

181 W. J. Mulder, R. Koole, R. J. Brandwijk, G. Storm, P. T. Chin, G. J. Strijkers, C. de Mello Donega, K. Nicolay and A. W. Griffioen; "Quantum dots with a paramagnetic coating as a bimodal molecular imaging probe", Nano Lett 2006, 6(1) 1-6.

182 G. A. van Tilborg, W. J. Mulder, P. T. Chin, G. Storm, C. P. Reutelingsperger, K. Nicolay and G. J. Strijkers; "Annexin A5-conjugated quantum dots with a paramagnetic lipidic coating for the multimodal detection of apoptotic cells", Bioconjug Chem 2006, 17(4) 865-8.

183 R. Bakalova, Z. Zhelev, I. Aoki, H. Ohba, Y. Imai and I. Kanno; "Silica-shelled single quantum dot micelles as imaging probes with dual or multimodality", Anal Chem 2006, 78(16) 5925-32.

184 A. Dirksen, E. W. Meijer, W. Adriaens and T. M. Hackeng; "Strategy for the synthesis of multivalent peptide-based nonsymmetric dendrimers by native chemical ligation", Chem Commun (Camb) 2006, 15) 1667-9.

S. Wang, B. R. Jarrett, S. M. Kauzlarich and A. Y. Louie; "Core/shell quantum dots with high relaxivity and photoluminescence for multimodality imaging", J Am Chem Soc 2007, 129(13) 3848-56. nanoparticles for multimodal imaging", Angew Chem Int Ed Engl 2007, 46(20) 3680-2. 

Coll, L. V. Elst, R. Muller, S. Roux, P. Perriat and O. Tillement; "Hybrid gadolinium oxide nanoparticles: multimodal contrast agents for in vivo imaging", J Am Chem Soc 2007, 129(16) 5076-84.

188 C. W. Lai, Y. H. Wang, C. H. Lai, M. J. Yang, C. Y. Chen, P. T. Chou, C. S. Chan, Y. Chi, Y. C. Chen and J. K. Hsiao; "Iridium-complex-functionalized Fe3O4/SiO2 core/shell nanoparticles: a facile three-in-one system in magnetic resonance imaging, luminescence imaging, and photodynamic therapy", Small 2008, 4(2) 218-24.

189 M. R. Lisy, A. Hartung, C. Lang, D. Schuler, W. Richter, J. R. Reichenbach, W. A. Kaiser and I. Hilger; "Fluorescent bacterial magnetic nanoparticles as bimodal contrast agents", Invest Radiol 2007, 42(4) 235-41.

190 H. Gu, R. Zheng, H. Liu, X. Zhang and B. Xu; "Direct synthesis of a bimodal nanosponge based on FePt and ZnS", Small 2005, 1(4) 402-6.

191 F. A. Jaffer, M. Nahrendorf, D. Sosnovik, K. A. Kelly, E. Aikawa and R. Weissleder; "Cellular imaging of inflammation in atherosclerosis using magnetofluorescent nanomaterials", Mol Imaging 2006, 5(2) 85-92.

192 J. H. Choi, F. T. Nguyen, P. W. Barone, D. A. Heller, A. E. Moll, D. Patel, S. A. Boppart and M. S. Strano; "Multimodal biomedical imaging with asymmetric single-walled carbon nanotube/iron oxide nanoparticle complexes", Nano Lett 2007, 7(4) 861-7.

193 T. Amemiya, H. Nakajima, T. Katoh, H. Rakue, M. Miyagi and C. Ibukiyama; "Photodynamic therapy of atherosclerosis using YAG-OPO laser and Porfimer sodium, and comparison with using argon-dye laser", Jpn Circ J 1999, 63(4) 288-95.

194 Z. Chen, K. W. Woodburn, C. Shi, D. C. Adelman, C. Rogers and D. I. Simon; "Photodynamic Therapy With Motexafin Lutetium Induces Redox-Sensitive Apoptosis of Vascular Cells", Arterioscler Thromb Vasc Biol 2001, 21(5) 759-764.

195 T. M. Chou, K. W. Woodburn, W. F. Cheong, S. A. Lacy, K. Sudhir, D. C. Adelman and D. Wahr; "Photodynamic therapy: applications in atherosclerotic vascular disease with motexafin lutetium", Catheter Cardiovasc Interv 2002, 57(3) 387-94.

196 S. Kim, T. Y. Ohulchanskyy, H. E. Pudavar, R. K. Pandey and P. N. Prasad; "Organically modified silica nanoparticles co-encapsulating photosensitizing drug and aggregation-enhanced two-photon absorbing fluorescent dye aggregates for two-photon photodynamic therapy", J Am Chem Soc 2007, 129(9) 2669-75. 
197 M. Overhaus, J. Heckenkamp, S. Kossodo, D. Leszczynski and G. M. LaMuraglia; "Photodynamic therapy generates a matrix barrier to invasive vascular cell migration", Circ Res 2000, $86(3) 334-40$.

198 S. G. Rockson, D. P. Lorenz, W. F. Cheong and K. W. Woodburn; "Photoangioplasty: An emerging clinical cardiovascular role for photodynamic therapy", Circulation 2000, 102(5) 591-6.

199 T. Wakamatsu, T. Saito, J. Hayashi, T. Takeichi, K. Kitamoto and K. Aizawa; "Long-term inhibition of intimal hyperplasia using vascular photodynamic therapy in balloon-injured carotid arteries", Med Mol Morphol 2005, 38(4) 225-32.

200 S. Link and M. A. El-Sayed; "Shape and size dependence of radiative, non-radiative and photothermal properties of gold nanocrystals", International Reviews in Physical Chemistry 2000, 19(3) $409-453$.

201 N. W. S. Kam, M. O'Connell, J. A. Wisdom and H. Dai; "Carbon nanotubes as multifunctional biological transporters and near-infrared agents for selective cancer cell destruction", Proc Natl Acad Sci U S A 2005, 102(33) 11600-5.

J. D. Gibson, B. P. Khanal and E. R. Zubarev; "Paclitaxel-functionalized gold nanoparticles", J Am Chem Soc 2007, 129(37) 11653-61.

G. F. Paciotti, L. Myer, D. Weinreich, D. Goia, N. Pavel, R. E. McLaughlin and L. Tamarkin; "Colloidal gold: a novel nanoparticle vector for tumor directed drug delivery", Drug Deliv 2004, 11(3) 169-83.

M. Shimada, S. Natsugoe and T. Aikou; "Enhanced efficacy of Bleomycin adsorbed on silica particles against lymph node metastasis derived from a transplanted tumor", Anticancer Res 1995, 15(1) 109-15.

S. Vallabhajosula; "(18)F-labeled positron emission tomographic radiopharmaceuticals in oncology: an overview of radiochemistry and mechanisms of tumor localization", Semin Nucl Med 2007, 37(6) 400-19.

H. Alencar, M. A. Funovics, J. Figueiredo, H. Sawaya, R. Weissleder and U. Mahmood; "Colonic adenocarcinomas: near-infrared microcatheter imaging of smart probes for early detection-study in mice", Radiology 2007, 244(1) 232-8.

C. Bremer, C. H. Tung and R. Weissleder; "Molecular imaging of MMP expression and therapeutic MMP inhibition", Acad Radiol 2002, 9 Suppl 2(S314-5.

208 M. L. Balestrieri and C. Napoli; "Novel challenges in exploring peptide ligands and corresponding tissue-specific endothelial receptors", Eur J Cancer 2007, 43(8) 1242-50. 
209 M. Palmowski, J. Huppert, G. Ladewig, P. Hauff, M. Reinhardt, M. M. Mueller, E. C. Woenne, J. W. Jenne, M. Maurer, G. W. Kauffmann, W. Semmler and F. Kiessling; "Molecular profiling of angiogenesis with targeted ultrasound imaging: early assessment of antiangiogenic therapy effects", Mol Cancer Ther 2008, 7(1) 101-9.

210 M. F. Corsten, L. Hofstra, J. Narula and C. P. Reutelingsperger; "Counting heads in the war against cancer: defining the role of annexin A5 imaging in cancer treatment and surveillance", Cancer Res 2006, 66(3) 1255-60.

211 N. Thapa, S. Kim, I. So, B. Lee, I. Kwon, K. Choi and I. Kim; "Discovery of a phosphatidylserinerecognizing peptide and its utility in molecular imaging of tumour apoptosis", Journal of CelIular and Molecular Medicine 2008, 12(5a) 1649-1660. 
Chapter 3 


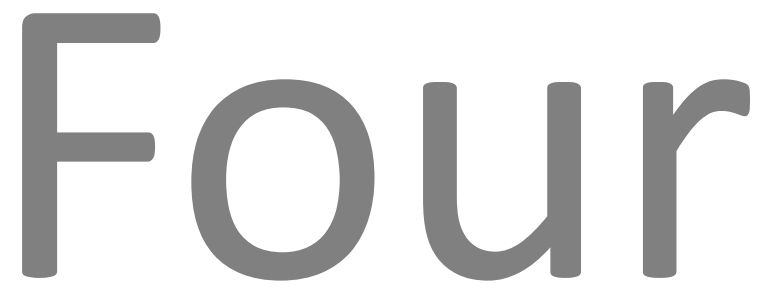

\section{Optical and magnetic resonance imaging of cell death and platelet activation using} Annexin A5-functionalized quantum dots

Nano Letters 2007, 7(1) p93-100

L Prinzen, RJJHM Miserus, A Dirksen, TM Hackeng, N Deckers, N Bitsch, RTA Megens, K Douma, JW Heemskerk, ME Kooi, PM Frederik, DW Slaaf, MAMJ van Zandvoort, CPM Reutelingsperger 


\section{Chapter 4}

\section{Abstract}

A quantum dot-based nanoparticle is presented, allowing visualization of cell death and activated platelets with fluorescence imaging and MRI. The particle exhibits intense fluorescence and a large MR relaxivity $\left(r_{1}\right)$ of $3000-4500 \mathrm{mM}^{-1} \mathrm{~s}^{-1}$ per nanoparticle, due to a newly designed construct increasing the gadolinium-DTPA load. The nanoparticle is suitable for both anatomic and sub-cellular imaging of structures in the vessel wall and is a promising bimodal contrast-agent for future in vivo imaging studies. 


\section{Introduction}

Contrast-enhancing nanoparticles are essential in the field of molecular imaging. Molecular imaging focuses on early detection of various diseases and supports personalized therapy. While conventional imaging modalities such as computer tomography and magnetic resonance imaging (MRI) allow visualization of anatomical and (patho)physiological consequences of the disease, molecular imaging is based on the molecular and cellular fingerprints of the disease, requiring disease-specific molecular targets as well as target-specific nanoparticles. Furthermore, these nanoparticles should allow visualization in vivo with sufficient perceptibility.

Programmed cell death (PCD) is a process of organized cell suicide, and occurs in various forms, of which apoptosis is the best known ${ }^{1}$. PCD plays important roles in physiology as well as pathology of multi-cellular organisms. During homeostasis a balance exists between cell proliferation and cell death. This equilibrium is disturbed under certain pathological conditions. Acute myocardial infarctions ${ }^{2}$, heart failure ${ }^{3}$, or unstable atherosclerotic plaques ${ }^{4,5}$ show increased PCD, whereas in cancer this balance shifts towards cell proliferation ${ }^{6}$. Anti-cancer therapies are being developed in which PCD is induced in cancer cells to reverse tumor growth ${ }^{7-9}$. Because of the role of PCD in numerous diseases, imaging of this process would be of great value to diagnosis and guidance of therapy.

One of the most explored molecular imaging targets of PCD is phosphatidylserine (PS). This aminophospholipid is translocated from the inner to the outer leaflet of the cellular membrane in dying cells, triggering phagocytosis ${ }^{10}$. Cell surface exposure of PS occurs during most types of cell death ${ }^{8}$, but is not limited to dying cells. For example, blood platelets expose PS in an advanced stage of activation ${ }^{11,12}$, providing a physiological surface for blood coagulation processes. Hence, molecular imaging of PS can also be of value to diagnosis and management of thrombotic diseases.

Annexin A5 (AnxA5) is a well explored molecular imaging probe to visualize cell surface exposure of PS ${ }^{13}$. AnxA5 binds to PS with a $\mathrm{K}_{d}$ in the $n M-r a n g e$ in the presence of $\mathrm{Ca}^{2+}$ ions ${ }^{14}$. It can be coupled to a variety of reporter compounds making it suitable to use in 


\section{Chapter 4}

combination with various imaging modalities including optical ${ }^{15,16}$, nuclear ${ }^{17,}{ }^{18}$, and magnetic resonance imaging (MRI) ${ }^{19,20}$. In vivo studies in animals and patients demonstrated that AnxA5 can be used to non-invasively detect the vulnerability of atherosclerotic plaques ${ }^{21,22}$, size and location of the infarct region in acute myocardial infarctions ${ }^{2}$, the extent of liver apoptosis ${ }^{23,24}$, and the localization of mural thrombi in abdominal aortic aneurisms ${ }^{25}$. In addition, imaging cell death with AnxA5 allows early assessment of the efficacy of anti-cancer therapy ${ }^{9}$. These studies utilized radioactively labeled AnxA5 in combination with single photon emission computed tomography (SPECT) or positron emission tomography (PET). SPECT and PET imaging do not yield anatomical information, and have a relatively poor resolution: $8-14 \mathrm{~mm}$ for SPECT and 4-7 $\mathrm{mm}$ for PET ${ }^{17,26}$. These limitations can be overcome by MRI using targeted contrast-agents. MRI has a better resolution $( \pm 300 \mu \mathrm{m}){ }^{26}$ and provides anatomical information, enabling a more accurate localization and characterization of the target site. However, sensitivity of this imaging modality for contrast-agents is still relatively low.

In this paper a new, AnxA5-functionalized and bimodal nanoparticle is presented that is suitable for MRI and fluorescence imaging allowing analysis at the anatomical as well as sub-cellular level. The nanoparticle is based on a quantum dot (QD), which is intensely fluorescent. The emission wavelength of QDs can be varied by altering the size of the semiconductor crystal core. Emission spectra of QDs are very narrow: emission peak width is about $20 \mathrm{~nm}$ at half maximum. Furthermore, QDs with different emission wavelengths have the same excitation wavelength. These properties allow multicolor analysis of samples in a single procedure ${ }^{27}$. A recent publication by Le Gac et al. ${ }^{28}$ showed that QDs (Quantum Dot Corporation, now Invitrogen) resist photo-bleaching and allow therefore imaging of fast, cellular processes over a longer period of time as compared to organic fluorescent dyes.

Long term in vivo effects of QDs are unknown so far. QDs used in this study are potentially toxic substances because the semiconductor crystal core contains cadmium and selenium. Toxicity of QDs depends on multiple factors, e.g. size, charge, concentration, outer coating bioactivity, and stability of the $Q D^{29}$. A recent study reported that mice tolerated intravenous injection of QDs well up to 133 days after administration. Neither 
signs of necrosis at sites of deposition nor signs of QD breakdown were observed ${ }^{30}$. The QDs used in that study are from the same supplier as the QDs of the current study.

The present study visualizes QDs by two-photon laser scanning microscopy (TPLSM). The laser power of TPLSM required to have sufficient QD fluorescence is considerably lower as compared to organic fluorescent dyes, due to the high quantum yield of QDs. This limits further potential photo-bleaching of the probe and photo-damage of the sample. Furthermore, high resolution images with low background fluorescence can be acquired. This is due to several factors: first, TPLSM excitation occurs only at the focal point preventing out-of-focus contribution ${ }^{31,32}$. Second, as indicated above, the required laser power is low, thereby also reducing background (auto)fluorescence. Third, because emission spectra of the QDs are narrow ${ }^{27}$, stringent emission filters can be applied to reduce background light as much as possible. Hence, it is possible to image structures hidden deeper in tissues more accurately with TPLSM without provoking tissue damage, for example atherosclerotic plaque imaging in arteries ${ }^{31}$.

\section{Materials and Methods}

Annexin A5: In order to accommodate conjugation chemistry for coupling AnxA5 to larger structures without impairing the PS binding property, a cysteine residue was engineered in the N-terminal tail, which is located apical to the PS binding sites (cys2AnxA5) ${ }^{33}$. Maleimide-activated biotin (Pierce, Rockford, IL) was coupled to cys2-AnxA5 according to the recommendations of the manufacturer. The cys2-M1234 variant, which does not bind PS ${ }^{34}$, was coupled to maleimide-activated biotin similarly and served as a negative control.

Gd-DTPA and Gd-DTPA wedge: Biotin was coupled to Gd-DTPA as described by Langereis et al. ${ }^{35}$; biotinylated Gd-wedge was synthesized similar to a method described previously ${ }^{36}$.

Functionalized quantum dots: AnxA5-QDs were prepared in a 1:1 stoichiometry by mixing equimolar amounts of streptavidin-coated QDs (stock concentration $1 \mu \mathrm{M}$, Invitrogen, Eugene, OR, with emission peaks at either 525 or $585 \mathrm{~nm}$ ) with biotinylated AnxA5 in binding buffer (10 mM HEPES, pH 7.4, containing $150 \mathrm{mM} \mathrm{NaCl}, 5 \mathrm{mM} \mathrm{KCl}, 1 \mathrm{mM}$ 


\section{Chapter 4}

$\mathrm{MgCl} 2,2.5 \mathrm{mM} \mathrm{CaCl} 2$ and $1 \mathrm{mg} / \mathrm{mL}$ Bovine Serum Albumin). The AnxA5-functionalized QDs were incubated with a minor excess of Gd-DTPA or Gd-wedge in order to saturate the unoccupied biotin binding sites of streptavidin. This procedure yields complexes with average stoichiometries of QD:AnxA5:Gd of 1:1:30 for AnxA5-QD-Gd and 1:1:240 for AnxA5-QD-Gd-wedge. These stoichiometries are derived from the information supplied by Invitrogen that each QD is coated with 8-12 streptavidin molecules.

Cryo-transmission electron microscopy: Specimen preparation for cryo-EM was done according to established procedures ${ }^{37}$. Briefly, a thin film was prepared from the sample in the Vitrobot ${ }^{\mathrm{TM}}$, an automated vitrification robot with temperature and humidity control (patented by the University of Maastricht; patent licensed to FEI company, Hillsboro, OR/USA). The thin film was formed by applying $3 \mu$ of the sample/suspension to a Quantifoil grid in the chamber of the Vitrobot at $22{ }^{\circ} \mathrm{C}$ and at a relative humidity $>97 \%$. Prior to sample application, the Quantifoil grid (R 2/2, Quantifoil $\mathrm{GmbH}$, Jena/BRD) was glow-discharged to make the surface hydrophilic. Excess liquid was blotted away (one blot for one second) and the thin film thus formed was shot into melting ethane. The grids with vitrified thin films were transferred to the microscope using a Gatan 626 cryo-transfer/cryo-holder system (Gatan, Pleasanton, CA, USA). Micrographs were taken with the specimen at $-170 \stackrel{\circ}{ } \mathrm{C}$, using low dose conditions in a CM12 transmission microscope (Philips, Eindhoven, The Netherlands) operating at 120 kV.

Cell culture, apoptosis stimulation, staining: Jurkat cells (ATCC, Middlesex, UK) were cultured in Roswell Park Memorial Institute (RPMI) 1640 medium (Gibco BRL), supplemented with $100 \mathrm{U} / \mathrm{mL}$ penicillin, $0.1 \mathrm{mg} / \mathrm{mL}$ streptomycin and $10 \%$ heat-inactivated fetal bovine serum, and incubated in a humidified atmosphere at $37{ }^{\circ} \mathrm{C}$ and $5 \% \mathrm{CO}_{2}$. Cells were split every two or three days to $10^{5} / \mathrm{mL}$. For experiments, cells were concentrated to $10^{6} / \mathrm{mL}$. Apoptosis was induced by adding anti-Fas (anti-human antibody, clone 7C11, Immunotech, Marseille, France) to the culture medium at a final concentration of $200 \mathrm{ng} / \mathrm{mL}$, and incubated in a humidified atmosphere at $37{ }^{\circ} \mathrm{C}$ and $5 \% \mathrm{CO}_{2}$ for three hours. Next, cells were washed with binding buffer, and stained, as indicated, with either of the two nanoparticles at a concentration of $40 \mathrm{nM}$ for 10 minutes. After- 
wards, cells were washed again with binding buffer and imaged with Two-Photon Laser Scanning Microscopy (TPLSM) or MRI.

For TPLSM experiments, cells were also incubated with a nucleic acid stain, either $2 \mu \mathrm{M}$ Syto41, a nucleic acid dye for viable and dead cells, (peak emission wavelength $450 \mathrm{~nm}$, stock concentration $5 \mathrm{mM}$, Invitrogen, Eugene, OR/USA), or $2.5 \mu \mathrm{g} / \mathrm{mL}$ Propidium lodide (peak emission wavelength $620 \mathrm{~nm}$, stock $250 \mu \mathrm{g} / \mathrm{mL}$, Molecular Probes, Leiden, the Netherlands) a nucleic acid dye for cells with a disrupted cellular membrane, i.e., dead cells. For internalization experiments, cells were incubated with 40 nM AnxA5-QD525$\mathrm{Gd}$, along with $200 \mathrm{ng} / \mathrm{mL}$ anti-Fas. After 5.5 hours cells were washed extensively in ethylene diamine tetra acetate (EDTA) to remove AnxA5 present on the outside of cells, and after washing with binding buffer, cells were stained with $40 \mathrm{nM}$ AnxA5-QD585-Gd. Next cells were washed with binding buffer and imaged with TPLSM.

Whole blood collection, clot formation: Blood was collected with informed consent from healthy volunteers by venapuncture and anticoagulated in 0.1 volume of $129 \mathrm{mM}$ trisodium citrate.

For TPLSM, clots were incubated with nanoparticles after clot-formation. Clot formation was induced by adding $15 \mathrm{mM} \mathrm{CaCl}_{2}$, and gentle mixing. Clots were allowed to form for one hour at $37{ }^{\circ} \mathrm{C}$ in coagulation tubes (BD Vacutainer SST II Advance, BD Diagnostics, Plymouth, UK). After coagulation, clots were washed in binding buffer, and next incubated with $40 \mathrm{nM}$ AnxA5-QD-Gd-wedge, prepared as described above, or with $500 \mathrm{nM}$ AnxA5-FITC (NeXins Research BV, Kattendijke, the Netherlands), for one hour at 37 ㅇ. Clots were also stained with Acridine Red (AR), a cytoplasm stain, (Chroma-Gesellschaft, Köngen, Germany) or $2 \mu \mathrm{M}$ Syto41. In one instance, whole blood was also preincubated with FITC-labeled anti-fibrinogen (anti-human antibody, WAK FA AF FITC-2, WAK Chemie Medical, Steinbach, Germany). Afterwards, clots were again washed in binding buffer and enclosed in $2 \%$ agarose gel for imaging.

For MRI, whole blood was incubated with $40 \mathrm{nM}$ or $200 \mathrm{nM}$ AnxA5-QD525-Gd-wedge prior to clot formation to label as many platelets as possible, because incubation of the clots after formation did not result in sufficient labeling for detection. To compensate for the anticoagulant effect of AnxA5, whole-blood was incubated with thrombin and 


\section{Chapter 4}

collagen, triggering additional PS-exposure on platelets. Clot-formation was induced by adding $15 \mathrm{mM} \mathrm{CaCl}_{2}, 1 \mathrm{nM}$ thrombin, and $20 \mu \mathrm{g} / \mathrm{mL}$ collagen in the presence of either 40 or 200 nM AnxA5-QD-Gd-wedge. Clots were allowed to form for one hour at $37^{\circ} \mathrm{C}$ in coagulation tubes as described above. Clots were placed in $2 \%$ agarose gel without washing, to minimize the wash out of labeled platelets.

Wire-injured carotid artery model: An 18-week old male Swiss mouse was anesthetized by pentobarbital (110 $\mathrm{mg} / \mathrm{kg}$ i.p.). The carotid artery was exposed while keeping it moist with saline and ligated at three sites: one proximal to the bifurcation of the artery and two distal to the bifurcation (one on each branch of the carotid artery). A flexible wire of $0.53 \mathrm{~mm}$ diameter was inserted in the common carotid artery via the external carotid artery and traversed three times to remove or damage the endothelium. Next, the blood flow was restored in the common carotid and internal branch; the external branch was tied off proximally of the incision with a fourth ligation ${ }^{38} .45$ minutes after injury the mouse was sacrificed by dissection of the diaphragm and cardiac perfusion with $5 \mathrm{~mL}$ of binding buffer in order to remove blood from the vasculature via liverpuncture. Then the carotid arteries were carefully excised, and connective and adipose tissue were removed. Next, the arteries were mounted into a home-built perfusion chamber ${ }^{39}$ (IDEE BV, Maastricht, the Netherlands) in Hanks Balanced Salt Solution (HBSS, pH 7.4), containing $144 \mathrm{mM} \mathrm{NaCl}, 14.9 \mathrm{mM}$ HEPES, $5.5 \mathrm{mM}$ glucose, $4.7 \mathrm{mM} \mathrm{KCl}$, $2.5 \mathrm{mM} \mathrm{CaCl}_{2}, 1.2 \mathrm{mM} \mathrm{KH}_{2} \mathrm{PO}_{4}$, and $1.2 \mathrm{mM} \mathrm{MgSO}_{4}$. The artery was slowly perfused with $200 \mathrm{nM}$ AnxA5-QD-Gd-wedge (500 $\mu \mathrm{L}$ in 30 minutes) using a microinjection pump (Predicor Infors, Basel, Switzerland). To stain cell nuclei and elastin in the vascular wall, the arteries were perfused with $500 \mu \mathrm{L}$ HBSS containing syto41 (final concentration $2 \mu \mathrm{M}$ ) and eosin (final concentration $0.5 \mu \mathrm{M}$ ) in a similar way, while simultaneously removing unbound nanoparticles. A static transmural pressure of $40 \mathrm{mmHg}$ was applied (using a modified Big Ben sphygmomanometer, Riester, Germany), and the arteries were imaged with TPLSM. Afterwards, the arteries were removed from the perfusion chamber and embedded in $2 \%$ agarose gel, and imaged with MRI.

Two-photon laser scanning microscopy: A standard Bio-Rad 2100MP (Hemel Hampstead, UK) was used in TPLSM mode. The excitation source was a Spectra Physics Tsunami Ti:Sapphire laser (Mountain View, CA/USA), tuned and mode-locked at $800 \mathrm{~nm}$. 
The Tsunami laser produces light pulses of about $140 \mathrm{fs}$ width at a repetition rate of 82 $\mathrm{MHz}$. Laser light reached the sample through the Nikon water immersion lenses $(60 \mathrm{x}$ water dipping, numerical aperture (NA) 1.0, working distance (WD) $2 \mathrm{~mm}$ or 60x water immersion, NA 1.2 and WD $0.22 \mathrm{~mm}$ ), incorporated in an upright Nikon E600FN microscope (Tokyo, Japan). Further magnification was achieved by optical zoom. Three photomultipliers detected the fluorescence. Each photomultiplier accepted a different, tunable wavelength region, which were color-coded: blue (for Syto41, 420-470 nm); green (for QD525 and FITC 510-540 nm); and red (for QD585 570-600 nm, for PI $580 \mathrm{~nm}$ and higher, and for acridine red $590 \mathrm{~nm}$ and higher). The three obtained images were combined into a single image. Only combined images are shown. No additional image processing was performed. An imaging speed of $0.1 \mathrm{~Hz}$ with a pixel dwell time of $39 \mu \mathrm{s}$ was used, while power at the sample was between 0.25 and $2.25 \mathrm{~mW}$.

Cells were imaged by applying $20 \mu \mathrm{L}$ of buffer containing cells on a microscope slide, and covering it by a coverslip. Blood clots were imaged while enclosed in $2 \%$ agarose gel, covered with binding buffer.

Magnetic resonance imaging: MRI was performed at room temperature with a $1.5 \mathrm{~T}$ whole-body system (Intera, Philips Medical Systems, Best, the Netherlands) using an inversion recovery, turbo spin echo (IR-TSE) MR pulse sequence for visualization of Jurkat cells and whole-blood clots. Murine carotid arteries were imaged at room temperature with a Bruker Biospec 7.0T scanner (Bruker Biospin GmbH, Ettlingen, Germany) using a multi-slice spin echo sequence.

Jurkat cell samples were imaged using a $23 \mathrm{~mm}$ diameter surface radio-frequency coil (Philips Medical Systems, Best, the Netherlands). The imaging parameters for the single slice IR-TSE sequence were: repetition time (TR), $1580 \mathrm{~ms}$; inversion time (TI), $546 \mathrm{~ms}$; echo time (TE), $13 \mathrm{~ms}$; field-of-view (FOV), 40x40 mm²; matrix size, 192x192; slice thickness, $1.5 \mathrm{~mm}$; echo train length, 6; number of signal averages (NSA), 16. The obtained in-plane resolution was $208 \times 208 \mu^{2}$.

Imaging of whole-blood clots was performed using a $47 \mathrm{~mm}$ diameter surface radiofrequency coil (Philips Medical Systems, Best, the Netherlands) and slightly different parameters for the IR-TSE sequence: TR, $1500 \mathrm{~ms}$; TI, $610 \mathrm{~ms}$; TE, $13 \mathrm{~ms}$; FOV, 40x40 


\section{Chapter 4}

$\mathrm{mm}^{2}$; matrix size, 192x192; slice thickness, $1.5 \mathrm{~mm}$; echo train length, 6; NSA, 32. The obtained in-plane resolution was $208 \times 208 \mu \mathrm{m}^{2}$.

Carotid images were obtained using a $35 \mathrm{~mm}$ diameter quadrature coil (Bruker Biospin $\mathrm{GmbH}$, Ettlingen, Germany) and a multi-slice spin echo sequence with the following parameters: TR, $800 \mathrm{~ms}$; TE, $10.2 \mathrm{~ms}$; FOV, $27.7 \times 27.7 \mathrm{~mm}^{2}$; matrix size, 312×312; slice thickness, $0.5 \mathrm{~mm}$; NSA, 125. The obtained in-plane resolution was $89 \times 89 \mu \mathrm{m}^{2}$.

Cells were imaged as a pellet in a PCR-eppendorf, while blood clots and mice carotid arteries were imaged enclosed in $2 \%$ agarose gel and covered by binding buffer to prevent dehydration.

In all images, regions of interest (ROIs) were drawn, and mean values and standard deviations were calculated using JiveX (version 4.1, VISUS Technology Transfer GmbH, Bochum, Germany) or Para-vision 4.0 (Bruker Biospin GmbH, Ettlingen, Germany). ROIs were placed in the center of the Jurkat cell samples and whole-blood clots. For the analysis of the carotid arteries ROls were placed over the entire vessel wall of the carotid arteries, and divided into quadrants to allow analysis and comparison of different regions of the vascular wall.

\section{Results}

The first version of the nanoparticle consisted of biotinylated AnxA5 coupled to streptavidin coated QDs in a 1:1 stoichiometry. QDs had emission peaks of either $525 \mathrm{~nm}$ (QD525, green) or $585 \mathrm{~nm}$ (QD585, red). AnxA5 contained a single biotin, located apical to the PS binding sites. The remaining binding sites of streptavidin were saturated with biotinylated Gadolinium-DTPA ${ }^{35}$, an MRI contrast-agent (biotinylated Gd-DTPA, figure 1A; AnxA5-QD-Gd, figure 2A).

To increase the perceptibility of the nanoparticle in MRI, it was aimed to increase the load of Gd-DTPA. Therefore, a biotinylated construct was designed consisting of a ly-

sine-wedge with eight Gd-DTPA complexes attached to the periphery ${ }^{36}$ (Gd-wedge, figure 1B). Addition of this wedge to the described QDs resulted in the nanoparticle represented in figure $2 B$ ( $A n x A 5-Q D-G d-w e d g e)$. 


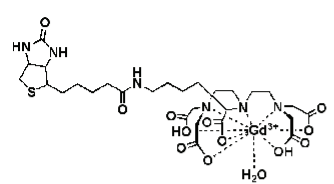

A<smiles>O=C1NC2CSCC2N1</smiles>

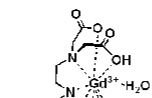

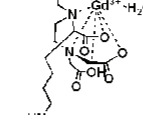

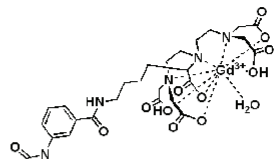

B

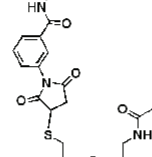

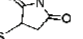<smiles>C1CCCCC1</smiles>

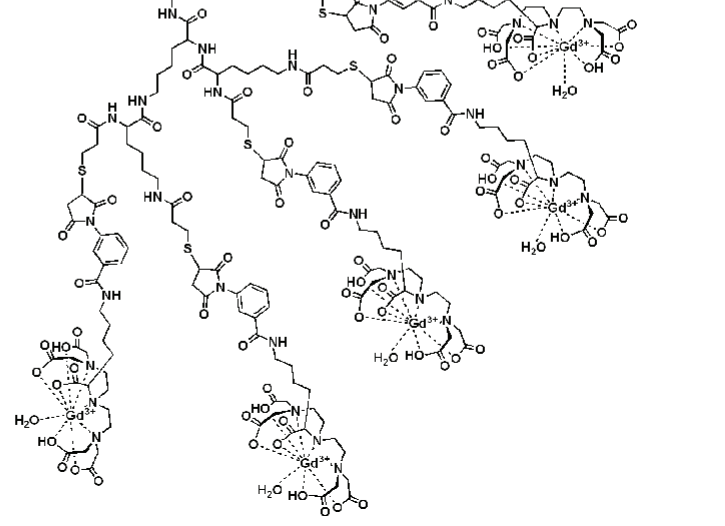

Figure 1. Molecular structure of the biotinylated Gd-DTPA (A) and biotinylated Gd-wedge (B) structures with biotin (red) and Gd-DTPA (blue). Color figure on page 181.

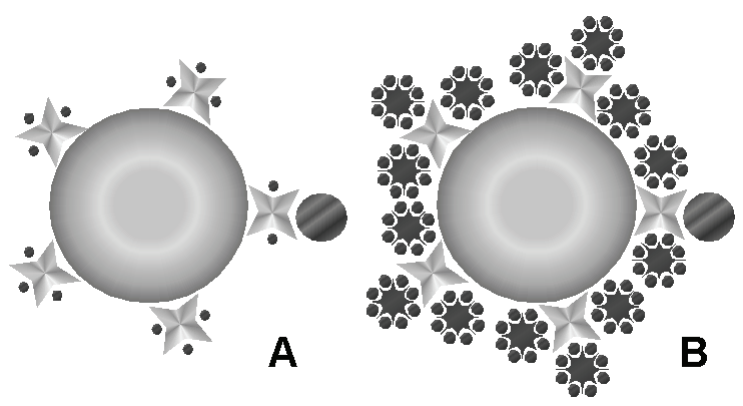

Figure 2. Schematic representation of the two nanoparticles. QDs contain approximately 1 AnxA5 and 10 streptavidin molecules. A: the nanoparticle with single biotinylated Gd-DTPA (AnxA5-QD-Gd); B: the nanoparticle with the biotinylated Gd-wedge, containing eight Gd-DTPA complexes each (AnxA5-QD-Gd-wedge). Green: QD, yellow: streptavidin, red dot: Gd-DTPA, red star: lysine-wedge, blue: AnxA5. Color figure on page 181. 


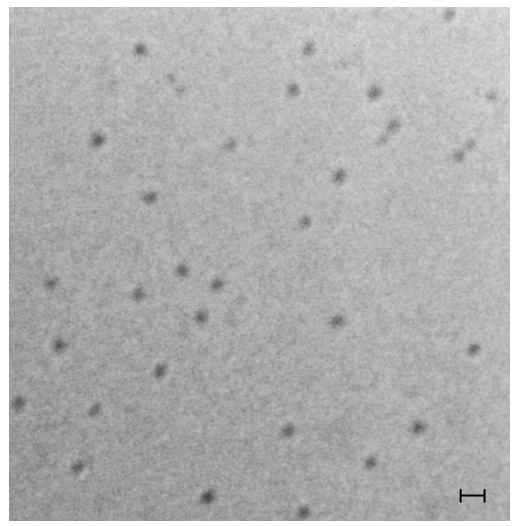

Figure 3. Cryo transmission electron microscopy image of AnxA5-QD525-Gd. Bar $=10 \mathrm{~nm}$.

Low temperature transmission electron microscopy (cryo-TEM, figure 3) showed that functionalized QDs in suspension were spherical, monodisperse, and non-aggregated. The spheric diameter was measured to be $6.7 \pm 1.0 \mathrm{~nm}$ (mean \pm standard deviation $(S D)), n=124$. AnxA5 and Gd-DTPA are not visible on the EM image; however, a gap of at least $1 \mathrm{~nm}$ between any two adjacent QDs indicates the presence of molecules on the surface of QDs. These results are similar to results in literature ${ }^{40}$.

In MRI, Gd-DTPA accelerates longitudinal relaxation ( $\mathrm{T}_{1}$-relaxation) which can be quantified by its longitudinal relaxivity $\left(r_{1}\right)$ expressed in $\mathrm{mM}^{-1} \mathrm{~s}^{-1}$. The $r_{1}$-values of AnxA5-QD-Gd and AnxA5-QD-Gd-wedge were estimated based on the $r_{1}$ per Gd-DTPA complex, which were subsequently multiplied by the number of Gd-DTPA complexes per nanoparticle. For biotinylated Gd-DTPA coupled to avidin $r_{1}$ was $17.5 \mathrm{mM}^{-1} \mathrm{~s}^{-1}$ per Gd-DTPA ${ }^{35}$, and for biotinylated Gd-wedge coupled to avidin $r_{1}$ was $15.6 \mathrm{mM}^{-1} \mathrm{~s}^{-1}$ per Gd-DTPA ${ }^{36}$. Based on information supplied by the manufacturer ( $\approx 10$ streptavidin molecules per $Q D$ ), it can be calculated that AnxA5-QD-Gd and AnxA5-QD-Gd-wedge have $r_{1}$-values per nanoparticle of $420-630 \mathrm{mM}^{-1} \mathrm{~s}^{-1}$ and $3000-4500 \mathrm{mM}^{-1} \mathrm{~s}^{-1}$, respectively.

AnxA5-QD-Gd and AnxA5-QD-Gd-wedge nanoparticles were examined on their property to discriminate between living and dying cells using TPLSM. Jurkat cells were triggered to execute apoptosis by activation of Fas receptor using anti-Fas antibodies. Binding of AnxA5-QD-Gd and AnxA5-QD-Gd-wedge occurred to early and late apoptotic cells but not to viable cells (figure 4) in a manner comparable to Oregon Green labeled AnxA5 (not shown) ${ }^{41}$. During early apoptosis, small patches of green fluorescence were 

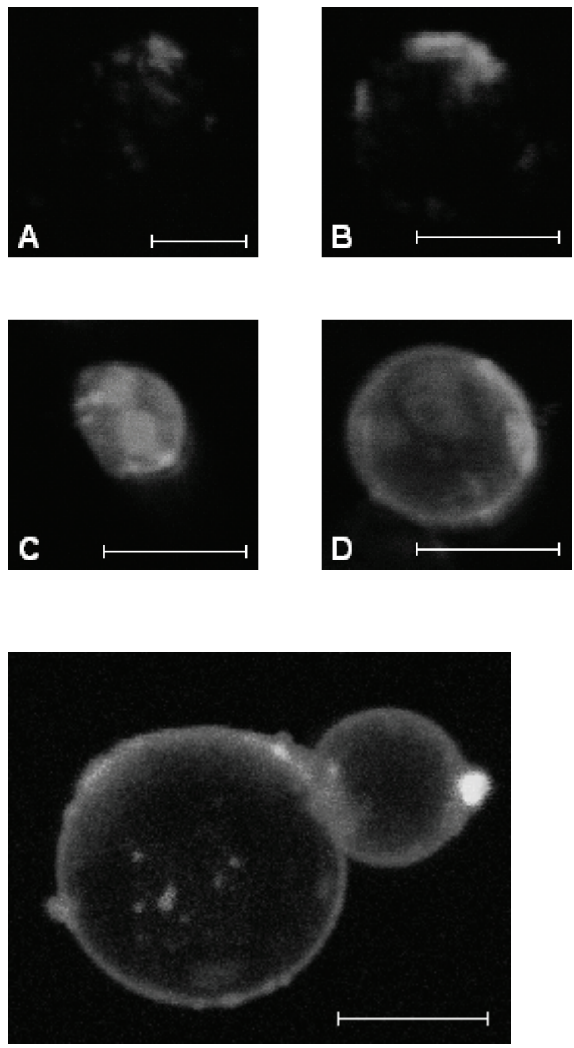

Figure 4. TPLSM images showing cellular AnxA5-QD525 (green) distribution. All cells were counterstained with $\mathrm{PI}$ (red) to determine membrane leakage, i.e., determine the stage of apoptosis. Top-row: early apoptotic Jurkat cells labeled with A; AnxA5-QD-Gd and B; AnxA5-QD-Gd-wedge. Bottom-row: late apoptotic cells labeled with C; AnxA5-QD-Gd and D; AnxA5-QD-Gdwedge. $B a r=10 \mu \mathrm{m}$. Color figure on page 182 .

visible on the cellular membrane (figure 4A/B), whereas during late apoptosis the entire cellular membrane was brightly fluorescent (figure 4C/D). Late apoptotic phase is characterized by loss of plasma membrane integrity and concomitant nuclear uptake of propidium iodide (PI), showing a red, fragmented nucleus. In addition, AnxA5-QD-Gd was able to be internalized by Jurkat cells (figure 5), as was reported for AnxA5 ${ }^{41}$. A movie, showing a z-stack of a cell, similar to the cell in figure 5, is also available at http://pubs.acs.org. Internalization of AnxA5-QD-Gd-wedge by Jurkat cells was not investigated in this study.

Binding of AnxA5-QD-Gd and AnxA5-QD-Gd-wedge to dying cells depends on cell surface exposure of PS, since QDs functionalized with M1234, a variant of AnxA5 that does not bind to PS ${ }^{34}$, do not show binding to apoptotic cells (figure 6). All together, these findings clearly demonstrate that AnxA5 coupled to a QD retains its full biological property to bind to PS exposed on the surface of dying cells. 

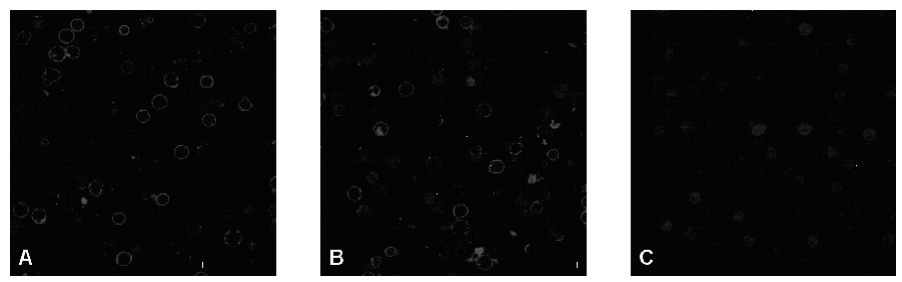

Figure 6. TPLSM images showing the cellular distributions of QD585 (red) coupled to AnxA5 or a mutant of AnxA5, unable to bind to PS (M1234), on apoptotic Jurkat cells. All cells were counterstained with Syto41 (blue), a nucleic acid stain. A: AnxA5-QD-Gd. B: AnxA5-QD-Gd-wedge. C: M1234-QD-Gd-wedge. Bar = $50 \mu \mathrm{m}$. Color figure on page 182.
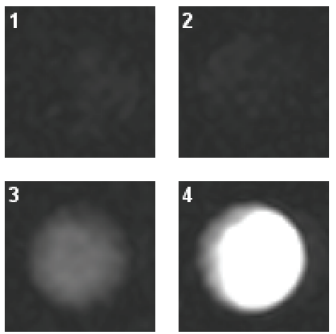

A

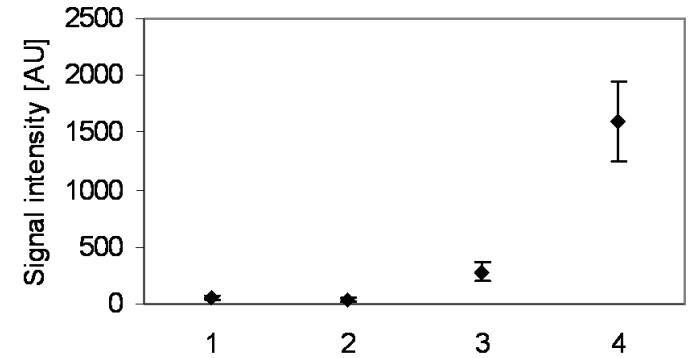

B

Figure 7. Sections of a single MR image, showing pellets of apoptotic Jurkat cells incubated with AnxA5-QD-Gd or AnxA5-QD-Gd-wedge. A: T1-weighted MR-images of the four cell samples. B: Graph showing mean \pm SD for signal intensities of the pixels in the regions of interest (ROIs), drawn within the samples shown in A. Cells incubated with: 1, buffer only (control 1); 2, $1.6 \mu \mathrm{M}$ Gd-wedge only (control 2); 3, $40 \mathrm{nM}$ AnxA5-QD-Gd; 4, $40 \mathrm{nM}$ AnxA5-QD-Gd-wedge.

Having established the AnxA5-specific binding properties of AnxA5-QD-Gd and AnxA5QD-Gd-wedge by fluorescence studies, the functional bimodality of the nanoparticle had to be confirmed. Apoptotic Jurkat cells were incubated with buffer (control 1), Gdwedge (control 2), AnxA5-QD-Gd or AnxA5-QD-Gd-wedge and subsequently measured with MRI (figure 7). The amount of Gd-wedge in sample 2 (control) was equal to the amount used in sample 4.

Cells incubated with AnxA5-QD-Gd (figure 7, sample 3) exhibit a signal intensity that is a factor 5.9 and 6.9 higher as compared to control 1 and control 2, respectively. Cells incubated with AnxA5-QD-Gd-wedge (4) exhibit a signal intensity that is a factor 5.8 higher than 3, demonstrating that AnxA5-QD-Gd-wedge has a much better $\mathrm{T}_{1}$-lowering 
effect than AnxA5-QD-Gd, as was expected from the differences in $r_{1}$. This results in a higher MRI signal intensity in $\mathrm{T}_{1}$-weighted imaging using the same amount of nanoparticles.

Cultured cells are simple in vitro systems for testing molecular imaging agents. Results derived from such systems hardly give reliable predictions about behavior in the in vivo situation. Therefore, a more complex system was chosen to assess the robustness of this nanoparticle as a bimodal molecular imaging agent. Since activated platelets expose PS, a human whole-blood clot system was chosen. Human whole blood was incubated with AnxA5-QD-Gd-wedge; subsequently PS exposure by platelets was induced to trigger clot-formation. The concentration of nanoparticles within the clot was expected to be much lower as compared to cell pellets. Within the clot only platelets expose PS, and not, for example, captured erythrocytes. An additional complexity of this system comes from the facts that AnxA5 competes with coagulation factors for binding to PS and acts as an anticoagulant preventing platelets from being incorporated into the thrombus ${ }^{11,42}$. Hence, MRI signal intensities were not expected to achieve the levels of figure 7. Therefore, following experiments were performed with AnxA5-QD-Gd-wedge only.

In order to label as many platelets as possible, whole blood was incubated with AnxA5QD-Gd-wedge prior to clot-formation. Coagulation in whole blood, mediated by thrombin generation and PS exposure on platelets, was triggered by adding $\mathrm{Ca}^{2+}$ to citrateanticoagulated whole blood. Additional PS exposure was triggered by pre-activating the platelets in whole blood with thrombin and collagen ${ }^{11}$, to counteract the anticoagulant effect of AnxA5. Whole-blood was incubated with buffer (control 1), Gd-wedge (control 2), $40 \mathrm{nM}$ or $200 \mathrm{nM}$ AnxA5-QD-Gd-wedge; subsequently clot-formation was induced and clots were measured with MRI (figure 8). The amount of Gd-wedge in sample 2 (control) was equal to the amount used in sample 4.

Signal intensities of the four clots are shown in figure 8B. Corresponding $\mathrm{T}_{1}$-values for clots $1,2,3$, and 4 are $734 \pm 85,740 \pm 44,678 \pm 89,628 \pm 30$ ms (mean \pm SD), respectively. The signal intensity of clot 4 was approximately $47 \%$ and $36 \%$ higher than controls 1 and 2, respectively. These results demonstrate that MRI of PS exposure in a more complex system is feasible using the AnxA5-QD-Gd-wedge nanoparticle. A recent study 

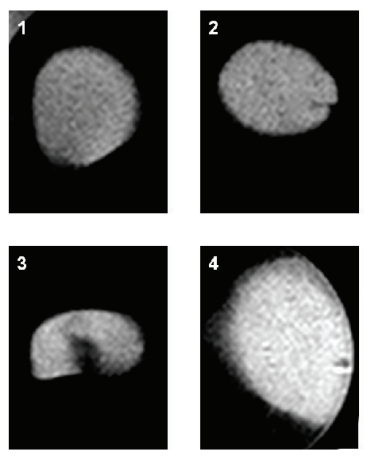

A

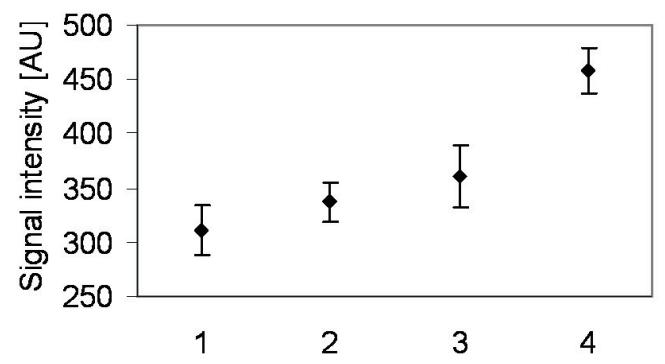

B

Figure 8. A: $\mathrm{T}_{1}$-weighted sections of a single MR image, showing whole-blood clots, activated with $\mathrm{Ca}^{2+}$, thrombin and collagen, and incubated with AnxA5-QD-Gd-wedge enclosed in 2\% agarose gel. B: graph showing mean $\pm \mathrm{SD}$ for the signal intensities of the pixels in ROls, drawn within the samples shown in A. Clots were incubated with: 1, buffer only (control 1); 2, $8 \mu \mathrm{M}$ Gd-wedge only (control 2); 3, $40 \mathrm{nM}$ AnxA5-QD-Gd-wedge; 4, $200 \mathrm{nM}$ AnxA5-QD-Gd-wedge. Clot 3 was slightly damaged, causing the black spot in the clot. Clot 4 was larger probably due to the fact that the higher concentration of AnxA5 inhibited clot retraction through its anticoagulant action.

showed that with SPECT imaging mural thrombus renewal in abdominal aortic aneurisms in vivo can be visualized using AnxA5 labeled with a radionuclide ${ }^{25}$. This study proved that thrombus imaging in vivo is feasible using AnxA5.

Whole-blood clots were also analyzed with TPLSM. For TPLSM, clots were incubated with AnxA5-QD-Gd-wedge after clot-formation, because in contrast to MRI, a lower amount of AnxA5-QD-Gd-wedge was sufficient for visualization by TPLSM. These experiments could thus be performed without interfering with clot-formation.

Distribution of AnxA5-QD-Gd-wedge in the whole-blood clots was similar to the distribution of FITC-labeled AnxA5 (figure 9A). AnxA5-QD-Gd-wedge specifically labeled activated platelets (figure $9 B / C$ ), as can be deduced from the size of the labeled structures, measuring ca. 1-2 $\mu \mathrm{m}$. Larger cells incorporated in the clot and easily discernible with a cytoplasm-specific or nucleic acid-specific dye, were stained neither with AnxA5-FITC nor AnxA5-QD-Gd-wedge (figure 9A/B). Figure 9C shows whole blood that was preincubated with anti-fibrinogen-FITC and subsequently activated to form a clot. Staining the clot with AnxA5-QD-Gd-wedge reveals that PS exposing platelets are aligned along the fibrin network within the clot. 

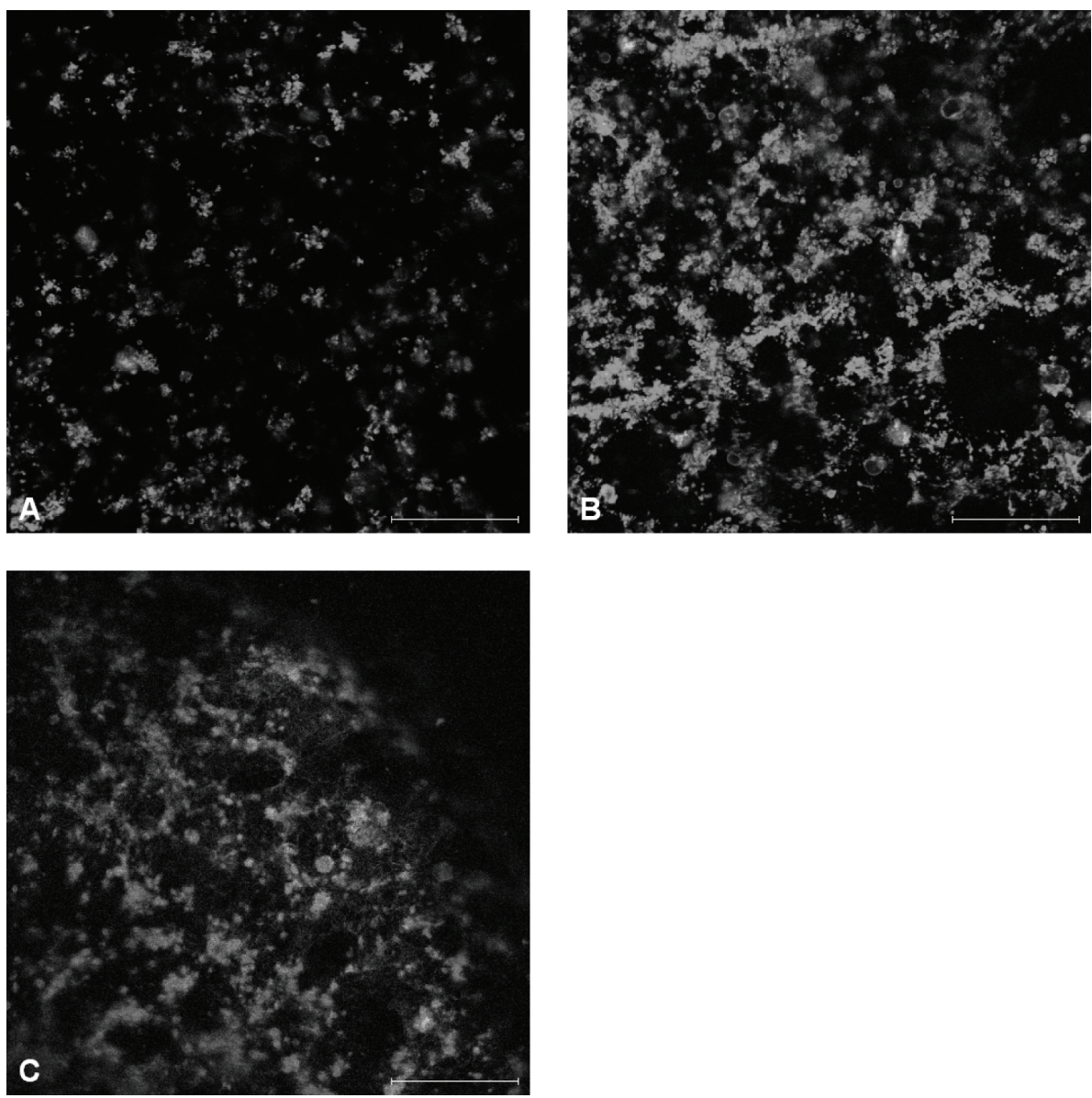

Figure 9: TPLSM images of whole-blood clots incubated with AnxA5 after clot-formation. A; green: AnxA5-FITC, blue: syto41, a nucleic acid stain; B; green: AnxA5-QD525-Gd, red: acridine red, a cytoplasmatic stain. C; red: AnxA5-QD585-Gd, green: anti-fibrinogen-FITC. Platelets show a bright cellular membrane, similar to apoptotic cells (compare to figures 4/5/6). Bar $=50 \mu \mathrm{m}$. Color figure on page 183.

To demonstrate the feasibility of this AnxA5-functionalized bimodal nanoparticle as a target-specific nanoparticle for cell death in the vascular wall, a murine carotid artery was mechanically injured in vivo by endothelial denudation using a metal wire as described elsewhere ${ }^{38}$. This injury results in rapid PS exposure by cells of the tunica intima, media, and adventitia ${ }^{38}$. Both damaged and undamaged carotid arteries were excised and mounted in a perfusion chamber. A static transmural pressure of $40 \mathrm{mmHg}$ was applied, and the arteries were labeled intraluminally with AnxA5-QD-Gd-wedge by 


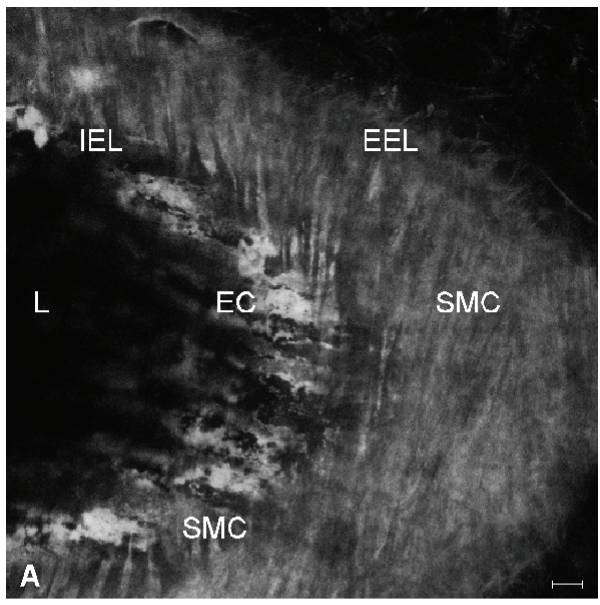

Figure 10: A; TPLSM image of a wire-injured murine carotid artery, showing the various vascular wall layers. The tunica intima consists of endothelial cells (ECs); the tunica media mainly consists of smooth muscle cells (SMCs). Uptake of nanoparticles is visible in ECs and SMCs. Green: AnxA5-QD-Gd-wedge; red: eosin, labeling elastin laminae; blue, syto41, labeling cell nuclei. L: lumen; IEL: internal elastic lamina; EEL: external elastic lamina. Bar $=10 \mu \mathrm{m}$. The imaging plane was slightly oblique, as opposed to a longitudinal direction of the artery. B; transversal MR image of two murine carotid arteries: undamaged (control, left) and damaged (right, same artery as shown in A). Bar $=0.5 \mathrm{~mm}$. Color figure on page 184

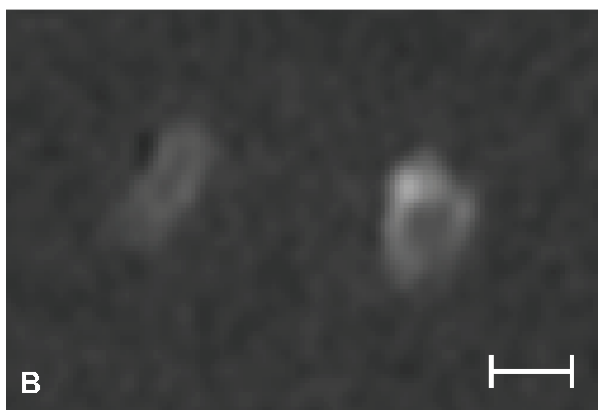

slow perfusion. The arteries were imaged by TPLSM as described in a recent article ${ }^{39}$. Figure 10A shows uptake of AnxA5-QD-Gd-wedge in the endothelial cells (ECs) in the tunica intima and in smooth muscle cells (SMCs) in the tunica media. Apparently, nanoparticles are able to penetrate through elastic laminae deep into the tunica media. It is unclear why the first SMC layer hardly shows any uptake of the nanoparticle, while the layer of SMCs between the outer two elastic laminae does show abundant labeling. Furthermore, no labeled platelets were visible, as no blood was present at time of labeling. A movie of a 3-dimensional representation of the carotid artery in figure $10 \mathrm{~A}$ is available at http://pubs.acs.org. The undamaged control artery hardly shows any labeling. Next, the arteries were removed from the perfusion chamber, and embedded in agarose gel for imaging with MRI. The amount of labeling of the damaged artery is sufficient to increase the MRI signal intensity compared to the control artery (figure 10B). To determine the increase in signal intensity, three transversal MRI slices of the carotid 
arteries were divided into quadrants. In these quadrants, ROIs were drawn to select the vascular wall. This is because the uptake of nanoparticle was not homogeneous throughout the vascular wall, as it was unevenly damaged by the wire. A total of twelve signal intensities was acquired per artery. The mean signal intensity increased from 83.2 \pm 3.6 for the control artery to $91.9 \pm 13.5$ for the damaged artery (arbitrary units, mean \pm SD), and ranging from 73.5 to 86.9 for the control artery and 73.4 to 125 for the damaged artery. This indicates that the increase in signal intensity differs per location in the vascular wall and ranges from 0 to $50 \%$ compared to the control, undamaged artery.

\section{Discussion}

Reports about QD-labeled AnxA5 have been published recently. As mentioned before, Le Gac et al. ${ }^{28}$ investigated photo-stability of streptavidin-coated, AnxA5-labeled QDs. In contrast to the biotinylated AnxA5 used in this study, the described AnxA5 contained three to four biotins, located at random sites on the AnxA5 polypeptide. This implies, as pointed out by the authors, that AnxA5 coupling to QDs may lead to masking of PS binding sites and aggregation of the QDs. The current study utilized cys2-AnxA5 which contains a single biotin attached to the $\mathrm{N}$-terminal tail that is located opposite to the side of the AnxA5 molecule harboring the PS binding sites ${ }^{43}$. Coupling of cys2-AnxA5 to streptavidin coated QDs neither induced aggregation nor caused a loss of biological activity. In addition, a recent study ${ }^{44}$ showed that cells, labeled with AnxA5-QDs, can be imaged using an in vivo imaging system.

Another study investigated bimodal, paramagnetic micellular QDs that were functionalized with AnxA5 ${ }^{45}$. Table 1 summarizes the most important characteristics of AnxA5functionalized bimodal nanoparticles published in literature. Two other $\mathrm{T}_{1}$-weighted nanoparticles, loaded with Gd-DTPA, are compared with the novel nanoparticle described in the current study. Furthermore, two $T_{2}$-weighted nanoparticles, containing iron oxide, are listed. AnxA5-QD-Gd-wedge has several advantages. First, it is the smallest nanoparticle so far. This can be advantageous when the imaging target is outside the vessel lumen, as in, for example, atherosclerotic plaques or tumors. Furthermore, of the $T_{1}$-weighted nanoparticles, AnxA5-QD-Gd-wedge is the nanoparticle with the highest relaxivity relative to the nanoparticle surface area. AnxA5-QD-Gd-wedge allows visi- 


\section{Chapter 4}

ble and near-infrared imaging and shows high fluorescence intensities. Additionally, QDs can be localized by electron microscopy ${ }^{40}$. Moreover, the $1: 1$ stoichiometry of the AnxA5-QD complex provides the most optimal configuration for maximizing the amount of bimodal nanoparticle bound to the PS exposing surface. Other advantages of AnxA5QD-Gd-wedge are straightforward preparation (add and mix), and variability of the nanoparticle's biological functionality. By replacing AnxA5 by a different biotinylated targeting function this nanoparticle can be used for molecular imaging of different biological processes ${ }^{46}$.

The results in this paper demonstrate that the bimodal AnxA5-QD-Gd-wedge nanoparticle can be used to analyze biological samples as well as vascular structures with MRI at the anatomical level and with TPLSM at the cellular level. AnxA5-QD-Gd-wedge has high potential for in vivo imaging in the near future.

\section{Acknowledgements}

We thank dr. Wim Engels for his assistance with the TPLSM experiments, and dr. Walter Backes for performing the preliminary MRI experiments. This work was financed by SenterNovem, BSIK 03033; TPLSM was financed by the Netherlands Organization for Scientific Research, NWO 902-16-276. 
Table 1. Comparison of five different bimodal AnxA5-conjugated nanoparticles. NIRF = near infrared fluorescence.

\begin{tabular}{|c|c|c|c|c|c|c|c|}
\hline & $\begin{array}{l}\text { nanopar- } \\
\text { ticle }\end{array}$ & $\begin{array}{l}T_{1} \text { or } T_{2^{-}} \\
\text {lowering }\end{array}$ & size $(n m)$ & $r_{1}$ & $r_{2}$ & $\begin{array}{l}\text { stoichiom } \\
\text { etry } \\
\text { AnxA5: } \\
\text { particle }\end{array}$ & optical \\
\hline 1 & $\begin{array}{l}\text { QD- } \\
\text { AnxA5- } \\
\text { Gd- } \\
\text { wedge }^{a}\end{array}$ & $\mathrm{~T}_{1}$ & $\sim 7$ & $\begin{array}{l}3000- \\
4500 \\
\left(\mathrm{mM}^{-1}\right. \\
\text { particle }) \mathrm{s}^{-}\end{array}$ & $\begin{array}{l}5600- \\
8400 \\
\left(\mathrm{mM}^{-1}\right. \\
\text { particle }) \mathrm{s}^{-}\end{array}$ & $1: 1$ & $\begin{array}{l}\text { Visible, } \\
\text { NIRF }\end{array}$ \\
\hline 2 & $\begin{array}{l}\text { Micellar } \\
\text { QD- } \\
\text { AnxA5 }\end{array}$ & $\mathrm{T}_{1}$ & $<10$ & $\begin{array}{l}\sim 1860 \\
\left(\mathrm{mM}^{-1}\right. \\
\text { particle }) \mathrm{s}^{-}\end{array}$ & $\begin{array}{l}\sim 2700 \\
\left(\mathrm{mM}^{-1}\right. \\
\text { particle }) \mathrm{s}^{-}\end{array}$ & $>5: 1^{e}$ & $\begin{array}{l}\text { Visible, } \\
\text { NIRF }\end{array}$ \\
\hline 3 & $\begin{array}{l}\text { AnxA5- } \\
\text { fluo- } \\
\text { rescein } \\
\text { lipo- } \\
\text { somes }^{c}\end{array}$ & $\mathrm{~T}_{1}$ & 100 & $\begin{array}{l}\sim 164,000 \\
\left(\mathrm{mM}^{-1}\right. \\
\text { particle }) \mathrm{s}^{-}\end{array}$ & $\begin{array}{l}\sim 272,000 \\
\left(\mathrm{mM}^{-1}\right. \\
\text { particle }) \mathrm{s}^{-}\end{array}$ & $>5: 1^{e}$ & Visible \\
\hline 4 & $\begin{array}{l}\text { AnxA5- } \\
\text { CLIO- } \\
\text { Cy5.5 }\end{array}$ & $\mathrm{T}_{2}$ & 50 & $\begin{array}{l}19\left(\mathrm{mM}^{-1}\right. \\
\mathrm{Fe}) \mathrm{s}^{-1}\end{array}$ & $\begin{array}{l}48\left(\mathrm{mM}^{-1}\right. \\
\mathrm{Fe}) \mathrm{s}^{-1}\end{array}$ & $3.5: 1$ & NIRF \\
\hline 5 & $\begin{array}{l}\text { AnxA5- } \\
\text { fluo- } \\
\text { rescein } \\
\text { micellar } \\
\text { iron } \\
\text { oxide }^{c}\end{array}$ & $\mathrm{~T}_{2}$ & 10 & $\begin{array}{l}13.3 \\
\left(\mathrm{mM}^{-1}\right. \\
\mathrm{Fe}) \mathrm{s}^{-1}\end{array}$ & $\begin{array}{l}159.6 \\
\left(\mathrm{mM}^{-1}\right. \\
\mathrm{Fe}) \mathrm{s}^{-1}\end{array}$ & $>5: 1^{e}$ & Visible \\
\hline
\end{tabular}

a, this paper; b, van Tilborg et al. ${ }^{45} ; c$, van Tilborg et $a .^{20}{ }^{20} d$, Schellenberger et al. ${ }^{19}$; e, personal communication. 


\section{References}

1 G. Kroemer, W. S. El-Deiry, P. Golstein, M. E. Peter, D. Vaux, P. Vandenabeele, B. Zhivotovsky, M. V. Blagosklonny, W. Malorni, R. A. Knight, M. Piacentini, S. Nagata and G. Melino; "Classification of cell death: recommendations of the Nomenclature Committee on Cell Death", Cell Death Differ 2005, 12 Suppl 2(1463-7.

L. Hofstra, I. H. Liem, E. A. Dumont, H. H. Boersma, W. L. van Heerde, P. A. Doevendans, E. De Muinck, H. J. Wellens, G. J. Kemerink, C. P. Reutelingsperger and G. A. Heidendal; "Visualisation of cell death in vivo in patients with acute myocardial infarction", Lancet 2000, 356(9225) 209-12.

J. Narula, N. Haider, R. Virmani, T. G. DiSalvo, F. D. Kolodgie, R. J. Hajjar, U. Schmidt, M. J. Semigran, G. W. Dec and B. A. Khaw; "Apoptosis in myocytes in end-stage heart failure", N Engl J Med 1996, 335(16) 1182-9.

J. M. Isner, M. Kearney, S. Bortman and J. Passeri; "Apoptosis in human atherosclerosis and restenosis", Circulation 1995, 91(11) 2703-11.

Y. J. Geng and P. Libby; "Evidence for apoptosis in advanced human atheroma. Colocalization with interleukin-1 beta-converting enzyme", Am J Pathol 1995, 147(2) 251-66.

G. I. Evan and K. H. Vousden; "Proliferation, cell cycle and apoptosis in cancer", Nature 2001, 411(6835) 342-8.

W. Hu and J. J. Kavanagh; "Anticancer therapy targeting the apoptotic pathway", Lancet Oncol 2003, 4(12) 721-9.

M. F. Corsten, L. Hofstra, J. Narula and C. P. Reutelingsperger; "Counting heads in the war against cancer: defining the role of annexin A5 imaging in cancer treatment and surveillance", Cancer Res 2006, 66(3) 1255-60.

R. L. Haas, D. de Jong, R. A. Valdes Olmos, C. A. Hoefnagel, I. van den Heuvel, S. F. Zerp, H. Bartelink and M. Verheij; "In vivo imaging of radiation-induced apoptosis in follicular lymphoma patients", Int J Radiat Oncol Biol Phys 2004, 59(3) 782-7.

J. Savill and V. Fadok; "Corpse clearance defines the meaning of cell death", Nature 2000, 407(6805) 784-788.

P. Thiagarajan and J. F. Tait; "Binding of annexin V/placental anticoagulant protein I to platelets. Evidence for phosphatidylserine exposure in the procoagulant response of activated platelets", J Biol Chem 1990, 265(29) 17420-3. 

M. G. oude Egbrink, B. Nieswandt and J. W. Heemskerk; "The glycoprotein VI-phospholipase Cgamma2 signaling pathway controls thrombus formation induced by collagen and tissue factor in vitro and in vivo", Arterioscler Thromb Vasc Biol 2005, 25(12) 2673-8.

H. H. Boersma, B. L. Kietselaer, L. M. Stolk, A. Bennaghmouch, L. Hofstra, J. Narula, G. A. Heidendal and C. P. Reutelingsperger; "Past, present, and future of annexin a5: from protein discovery to clinical applications", J Nucl Med 2005, 46(12) 2035-50.

C. P. Reutelingsperger and W. L. van Heerde; "Annexin V, the regulator of phosphatidylserinecatalyzed inflammation and coagulation during apoptosis", Cell Mol Life Sci 1997, 53(6) 52732.

M. van Engeland, L. J. Nieland, F. C. Ramaekers, B. Schutte and C. P. Reutelingsperger; "Annexin V-affinity assay: a review on an apoptosis detection system based on phosphatidylserine exposure", Cytometry 1998, 31(1) 1-9.

S. M. van den Eijnde, A. J. Luijsterburg, L. Boshart, C. I. De Zeeuw, J. H. van Dierendonck, C. P. Reutelingsperger and C. Vermeij-Keers; "In situ detection of apoptosis during embryogenesis with annexin V: from whole mount to ultrastructure", Cytometry 1997, 29(4) 313-20. C. P. Reutelingsperger, E. Dumont, P. W. Thimister, H. van Genderen, H. Kenis, S. van de Eijnde, G. Heidendal and L. Hofstra; "Visualization of cell death in vivo with the annexin A5 imaging protocol", J Immunol Methods 2002, 265(1-2) 123-32.

J. Toretsky, A. Levenson, I. N. Weinberg, J. F. Tait, A. Uren and R. C. Mease; "Preparation of F18 labeled annexin V: a potential PET radiopharmaceutical for imaging cell death", Nucl Med Biol 2004, 31(6) 747-52.

E. A. Schellenberger, D. Sosnovik, R. Weissleder and L. Josephson; "Magneto/optical annexin V, a multimodal protein", Bioconjug Chem 2004, 15(5) 1062-7.

G. A. van Tilborg, W. J. Mulder, N. Deckers, G. Storm, C. P. Reutelingsperger, G. J. Strijkers and K. Nicolay; "Annexin A5-functionalized bimodal lipid-based contrast agents for the detection of apoptosis", Bioconjug Chem 2006, 17(3) 741-9.

L. L. Johnson, L. Schofield, T. Donahay, N. Narula and J. Narula; "99mTc-annexin V imaging for in vivo detection of atherosclerotic lesions in porcine coronary arteries", J Nucl Med 2005, 46(7) 1186-93. and J. Narula; "Noninvasive detection of plaque instability with use of radiolabeled annexin A5 in patients with carotid-artery atherosclerosis", N Engl J Med 2004, 350(14) 1472-3. 


\section{Chapter 4}

and J. Zweit; "Imaging apoptosis in vivo using 124I-annexin V and PET", Nucl Med Biol 2005, 32(4) 395-402.

F. G. Blankenberg, P. D. Katsikis, J. F. Tait, R. E. Davis, L. Naumovski, K. Ohtsuki, S. Kopiwoda, M. J. Abrams, M. Darkes, R. C. Robbins, H. T. Maecker and H. W. Strauss; "In vivo detection and imaging of phosphatidylserine expression during programmed cell death", Proc Natl Acad Sci U S A 1998, 95(11) 6349-54.

L. Sarda-Mantel, M. Coutard, F. Rouzet, O. Raguin, J. M. Vrigneaud, F. Hervatin, G. Martet, Z. Touat, P. Merlet, D. Le Guludec and J. B. Michel; "99mTc-Annexin-V Functional Imaging of Luminal Thrombus Activity in Abdominal Aortic Aneurysms", Arterioscler Thromb Vasc Biol 2006, 26(9) 2153-9.

R. J. J. H. M. Miserus, S. Heeneman, J. M. A. v. Engelshoven, M. E. Kooi and M. J. A. P. Daemen; "Development and validation of novel imaging technologies to assist translational studies in atherosclerosis", Drug Disc. Today: Techn. 2006, 3(2) 195-204.

A. Watson, X. Wu and M. Bruchez; "Lighting up cells with quantum dots", Biotechniques 2003, 34(2) 296-300, 302-3.

S. LeGac, I. Vermes and A. vandenBerg; "Quantum Dots Based Probes Conjugated to Annexin V for Photostable Apoptosis Detection and Imaging", Nano Lett. 2006,

R. Hardman; "A toxicologic review of quantum dots: toxicity depends on physicochemical and environmental factors", Environ Health Perspect 2006, 114(2) 165-72.

B. Ballou, B. C. Lagerholm, L. A. Ernst, M. P. Bruchez and A. S. Waggoner; "Noninvasive imaging of quantum dots in mice", Bioconjug Chem 2004, 15(1) 79-86.

M. van Zandvoort, W. Engels, K. Douma, L. Beckers, M. Oude Egbrink, M. Daemen and D. W. Slaaf; "Two-photon microscopy for imaging of the (atherosclerotic) vascular wall: a proof of concept study", J Vasc Res 2004, 41(1) 54-63.

W. Denk, J. H. Strickler and W. W. Webb; "Two-photon laser scanning fluorescence microscopy", Science 1990, 248(4951) 73-6.

R. Huber, R. Berendes, A. Burger, M. Schneider, A. Karshikov, H. Luecke, J. Romisch and E. Paques; "Crystal and molecular structure of human annexin $V$ after refinement. Implications for structure, membrane binding and ion channel formation of the annexin family of proteins", J Mol Biol 1992, 223(3) 683-704. 
of crucial importance of domain I type I/ Ca2+-binding site in the mechanism of inhibition", J Biol Chem 1997, 272(16) 10474-82.

S. Langereis, H. A. Kooistra, M. H. van Genderen and E. W. Meijer; "Probing the interaction of the biotin-avidin complex with the relaxivity of biotinylated Gd-DTPA", Org Biomol Chem 2004, 2(9) 1271-3.

A. Dirksen, E. W. Meijer, W. Adriaens and T. M. Hackeng; "Strategy for the synthesis of multivalent peptide-based nonsymmetric dendrimers by native chemical ligation", Chem Commun (Camb) 2006, 15) 1667-9.

P. M. Frederik and D. H. Hubert; "Cryoelectron microscopy of liposomes", Methods Enzymol 2005, 391(431-48.

S. Ravassa, A. Bennaghmouch, H. Kenis, T. Lindhout, T. Hackeng, J. Narula, L. Hofstra and C. Reutelingsperger; "Annexin A5 Down-regulates Surface Expression of Tissue Factor: A NOVEL MECHANISM OF REGULATING THE MEMBRANE RECEPTOR REPERTOIR", J Biol Chem 2005, 280(7) 6028-35.

R. T. A. Megens, S. Reitsma, P. H. M. Schiffers, R. H. P. Hilgers, J. G. R. De Mey, D. W. Slaaf, M. G. A. oude Egbrink and M. A. M. J. van Zandvoort; "Two-Photon microscopy of vital murine elastic and muscular arteries. Combined structural and functional imaging with subcellular resolution", J Vasc Res 2006, in press(

B. N. Giepmans, T. J. Deerinck, B. L. Smarr, Y. Z. Jones and M. H. Ellisman; "Correlated light and electron microscopic imaging of multiple endogenous proteins using Quantum dots", Nat Methods 2005, 2(10) 743-9.

H. Kenis, H. van Genderen, A. Bennaghmouch, H. A. Rinia, P. Frederik, J. Narula, L. Hofstra and C. P. Reutelingsperger; "Cell surface-expressed phosphatidylserine and annexin A5 open a novel portal of cell entry", J Biol Chem 2004, 279(50) 52623-9.

S. Ramstrom, M. Ranby and T. L. Lindahl; "Platelet phosphatidylserine exposure and procoagulant activity in clotting whole blood--different effects of collagen, TRAP and calcium ionophore A23187", Thromb Haemost 2003, 89(1) 132-41.

R. Huber, M. Schneider, I. Mayr, J. Romisch and E. P. Paques; "The calcium binding sites in human annexin $V$ by crystal structure analysis at $2.0 \mathrm{~A}$ resolution. Implications for membrane binding and calcium channel activity", FEBS Lett 1990, 275(1-2) 15-21.

D. T. Dicker, S. H. Kim, Z. Jin and W. S. El-Deiry; "Heterogeneity in non-invasive detection of apoptosis among human tumor cell lines using annexin-V tagged with EGFP or Qdot-705", Cancer Biol Ther 2005, 4(9) 1014-7. 


\section{Chapter 4}

G. A. van Tilborg, W. J. Mulder, P. T. Chin, G. Storm, C. P. Reutelingsperger, K. Nicolay and G. J. Strijkers; "Annexin A5-Conjugated Quantum Dots with a Paramagnetic Lipidic Coating for the Multimodal Detection of Apoptotic Cells", Bioconjug Chem 2006, 17(4) 865-868.

46 A. Buehler, M. A. van Zandvoort, B. J. Stelt, T. M. Hackeng, B. H. Schrans-Stassen, A. Bennaghmouch, L. Hofstra, J. P. Cleutjens, A. Duijvestijn, M. B. Smeets, D. P. de Kleijn, M. J. Post and E. D. de Muinck; "cNGR: A Novel Homing Sequence for CD13/APN Targeted Molecular Imaging of Murine Cardiac Angiogenesis In vivo", Arterioscler Thromb Vasc Biol 2006, 


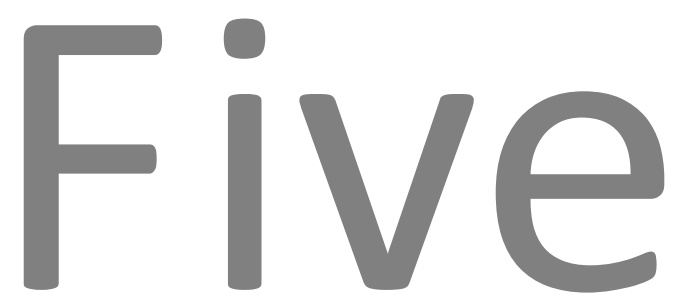

\section{Hot-spot labeling of apoptosis and} collagen in atherosclerotic plaques using

bimodal quantum dots

In preparation

L Prinzen, RJJHM Miserus, N Bitsch, TM Hackeng, ME Kooi, DW Slaaf, CPM

Reutelingsperger, MAMJ van Zandvoort 


\section{Chapter 5}

\section{Abstract}

Targeted bimodal quantum dots (Qdots) were utilized for imaging either collagen or cell death in atherosclerotic plaques of carotid arteries of ApoE-/- mice. Two-photon laser scanning microscopy (TPLSM) revealed that both types of Qdots bound to hot-spots in intimal as well as deeper layers of the atherosclerotic plaque. Likely, Qdots get access to vascular targets only at sites of endothelial damage, reflecting plaque instability. MRI showed a minor increase in signal intensity for collagen-targeting Qdots. 


\section{Introduction}

Molecular imaging is a fast growing research area. Specific molecular targeting of pathological sites by ligands coupled to contrast agents allows detection and diagnosis of disease using non-invasive imaging modalities such as MRI, PET, SPECT, ultrasound, $\mathrm{CT}$, or fluorescence imaging. Molecular imaging of atherosclerosis would allow noninvasive and accurate visualization of atherosclerotic plaques, as well as discrimination between stable and vulnerable plaques in patients at risk. Insight into biology of developing atherosclerotic plaques has provided potential targets such as matrix metalloproteinases ${ }^{1-3}$, macrophage markers ${ }^{4-6}$, and thrombus-specific molecular markers ${ }^{7,8}$.

Two promising targets are phosphatidylserine (PS) and collagen. PS is a phospholipid exposed on dying cells ${ }^{9}$ and activated platelets ${ }^{10}$. As these are both present in ruptured plaques or plaques at risk of rupture ${ }^{11}$, PS imaging may allow the detection of vulnerable plaques ${ }^{12,13}$. Annexin A5 (Anx) is a ligand for PS with an affinity in the nanomolar range $^{9,10}$, and is successfully used in several pre-clinical ${ }^{12,14,15}$ and clinical ${ }^{16-18}$ imaging studies on the detection of cell death.

More recently, collagen was proposed as a suitable target for imaging atherosclerosis ${ }^{19}$, ${ }^{20}$. Atherosclerotic plaques expose more collagen to the lumen compared to non-plaque areas of the vascular wall for two reasons: first, the intimal layer of the plaque is activated and possibly disrupted, which means that it is no longer a seamless cover of the collagen-containing basal lamina ${ }^{20}$. Second, as plaques become more advanced, a fibrous cap develops, consisting for a large part of collagen types I, III, IV, and $\mathrm{V}^{21}$. CNA35 (CNA) is a recently developed polypeptide that binds collagen types I, III, and IV with an affinity of $0.5 \mu \mathrm{M}^{22}$, and has shown to be a suitable probe for collagen targeting in atherosclerotic plaques in ApoE -/- mice ${ }^{19}$.

Recently, we reported a bimodal nanoparticle for molecular imaging. The nanoparticle consists of a streptavidin-coated quantum dot (Qdot, for fluorescence imaging) to which biotinylated Gadolinium-DTPA (for MR imaging) was coupled ${ }^{23}$. Bimodal imaging has the advantage of allowing non-invasive imaging by MRI and high-resolution imaging at subcellular level by fluorescence imaging of the same specimen. Qdots are fluores- 


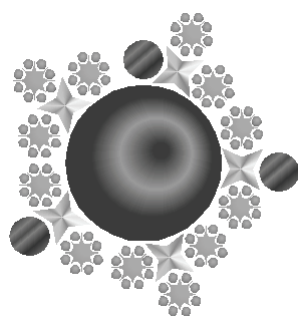

Figure 1 (left). Schematic representation of Anx- or CNA-Qdot. Red: Qdot; yellow: streptavidin; grey: Gd-DTPA8 wedge; blue: Anx or CNA. Color figure on page 184.

cent semiconductor nanocrystals, having several advantages over organic fluorophores: i) they have high quantum yields (i.e., they are brightly fluorescent) ${ }^{24}$, ii) low photobleaching ${ }^{25}$, allowing improved non-invasive ${ }^{26-28}$ and repetitive, long-term fluorescence imaging ${ }^{29}$, iii) they have broad excitation and narrow emission spectra, allowing simultaneous imaging of multiple targets using Qdots with different emission peaks ${ }^{30}$. These advantages render Qdots into attractive nanoparticles for in vivo molecular imaging, as demonstrated in imaging studies of tumor tissue ${ }^{31-33}$, sentinal lymph nodes ${ }^{34}$, and reticulo endothelial system (RES) ${ }^{35}$.

Qdots have previously been functionalized with additional contrast agents allowing magnetic resonance imaging (MRI) ${ }^{23,33,36}$ or positron emission tomography ${ }^{37}$. In the present study, we attempted to visualize vulnerable atherosclerotic plaques by both MRI and two-photon laser scanning microscopy (TPLSM). In our approach, we equipped streptavidin-conjugated Qdots with biotinylated Gadolinium-DTPA lysine-wedges, each containing 8 Gd-DTPA moieties for increased MRI sensitivity, as previously described ${ }^{23}$. This Qdot was further equipped with either biotinylated Anx or biotinylated CNA (both are polypeptides of approximately $35 \mathrm{kDa}$ ) for atherosclerotic plaque imaging (see figure 1).

First, biodistribution and pharmacokinetics of these bimodal CNA- and Anx-Qdots were determined in mice. Next, bimodal nanoparticles were investigated for their capability to detect atherosclerotic plaques in ApoE -/- mice. Atherosclerosis was induced by a high-fat diet and silicon perivascular collars around both carotid arteries ${ }^{38}$. Three or six weeks after collar placement, mice were scanned in vivo with MRI and injected with CNA-, Anx-, or control-Qdots. Afterwards, mice were sacrificed; carotid arteries were excised and imaged with TPLSM at sub-cellular resolution. 


\section{Materials and methods}

Annexin A5: A cysteine residue was engineered in the N-terminal tail, which is located apical to the PS binding sites (cys2-Anx). Maleimide-activated biotin (Pierce, Rockford, IL) was coupled to cys2-Anx according to the recommendations of the manufacturer.

CNA: CNA was produced as described previously ${ }^{22}$, and was biotinylated by coupling a C-terminal thioester on CNA to an $\mathrm{N}$-terminal cysteine residue on biotin using native chemical ligation.

Gd-DTPA wedge: Biotinylated Gd-wedge was synthesized similar to a method described previously ${ }^{23,39}$.

Bimodal Qdots: Anx-Qdots, CNA-Qdots, and control-Qdots were prepared as described previously ${ }^{23}$, by mixing streptavidin-coated QDots (stock concentration $1 \mu \mathrm{M}$ and emission peak at 525 or $585 \mathrm{~nm}$, Invitrogen, Eugene, OR/USA) with biotinylated Anx or CNA, and biotinylated Gd-wedge in Hanks Balanced Salt Solution (HBSS, pH 7.4), containing $144 \mathrm{mM} \mathrm{NaCl}, 14.9 \mathrm{mM}$ HEPES, $5.5 \mathrm{mM}$ glucose, $4.7 \mathrm{mM} \mathrm{KCl}, 2.5 \mathrm{mM} \mathrm{CaCl}$, $1.2 \mathrm{mM}$ $\mathrm{KH}_{2} \mathrm{PO}_{4}$, and $1.2 \mathrm{mM} \mathrm{MgSO}_{4}$. This procedure yields complexes with average stoichiometries of QD:Anx/CNA:Gd-wedge of 1:6:24. These stoichiometries were derived from information supplied by Invitrogen that each Qdot is coated with 8-12 streptavidin molecules. Control-Qdots lack both Anx and CNA.

Clearance and biodistribution study: Animal experiments were approved by the local ethics committee. Male Swiss mice $40.1 \pm 4.4 \mathrm{gr}$ (mean $\pm S D, n=1$ or 2 per group) were anesthetized by $3 \%$ isoflurane applied through a mask, anesthesia was maintained by $1.5 \%$ isoflurane. Mice were then canulated in the femoral vein for intravenous injection of Qdot585 solution (100 pmol Qdots per animal) and on the opposite side in the femoral artery for blood sample withdrawal. A blank blood sample was drawn prior to Qdot injection, and after injection samples were drawn $1^{\prime}, 2^{\prime}, 5^{\prime}, 10^{\prime}, 15^{\prime}, 20^{\prime}, 30^{\prime}, 45^{\prime}, 60^{\prime}$ and $90^{\prime}$ after injection. Samples of $50 \mu \mathrm{l}$ were drawn using a syringe, and kept at $4{ }^{\circ} \mathrm{C}$ until analysis. After 90' of circulation, mice were sacrificed by cervical dislocation, and liver, spleen, kidney, heart, lung, and intestine tissue were collected. To determine the fluorescence intensity in blood, samples were centrifuged for $3^{\prime}$ at $278 \mathrm{~g}$ and serum was collected. Whole-blood samples could not be measured due to the high absorbance of 


\section{Chapter 5}

erythrocytes. The residue did not contain Qdots, as was checked with TPLSM. Fluorescence intensities of blood samples were measured using a fluorospectrometer, the NanoDrop 3300 (NanoDrop Technologies, Inc, Wilmington, DE/USA). Two- $\mu$ l serum samples were applied to the NanoDrop pedestal and the fluorescence intensities were measured (arbitrary units) at the peak of the emission spectrum. In order to determine mono-exponential fits of the data and corresponding circulation half-lives, GraphPad Prism 4 (GraphPad Software, Inc.) was used. Figure 4 shows the clearance of Qdots normalized to the fluorescence intensity at $t=2^{\prime}$; intensity at $t=1^{\prime}$ was too variable (actual time of sample withdrawal could be between $0^{\prime}$ and $2^{\prime}$ ) due to too many handling steps in a short time.

Atherosclerotic mice: Experiments were approved by the local ethics committee. Fifteen-week old male ApoE -/- mice (Charles River Laboratories, Maastricht, the Netherlands), $\mathrm{n}=4$ per group, were fed a high-fat diet (Hope Farms diet 4021.06, Woerden, the Netherlands) for two weeks. After subcutaneous injection of buprenorphine (0.4 $\mathrm{mg} / \mathrm{kg}$ ), and while under isoflurane anesthesia applied through a mask, perivascular silicon collars (Standard Silicon Tubing, Helix Medical, Carpinteria, CA/USA; inner diameter $0.31 \mathrm{~mm}$, outer diameter $0.64 \mathrm{~mm}$, length $3 \mathrm{~mm}$ ) were placed bilaterally around the common carotid arteries and ligated using three ligatures, as described by Von der Thüsen et al. ${ }^{38}$, inducing atherosclerotic plaque growth proximal to the collar. Mice remained on high-fat diet. Either 3 or 6 weeks after collar placement, mice were again anesthetized by isoflurane, and received a cannula in the femoral vein. Three-week mice weighed $27.8 \pm 3.1 \mathrm{gr}($ mean \pm SD), six-week mice weighed $29.2 \pm 1.5 \mathrm{gr}$ (mean \pm SD) at time of cannulation. This cannula was approximately $40 \mathrm{~cm}$ in length, and already contained the Qdot525 solution. This length is necessary to allow injection from outside the MRI scanner without having to remove the mice from the scanner. This way, pre- and post-injection scans could be made of the carotid arteries at exactly the same position. The injected dose was 200 pmol Qdots per animal, which equals $\approx 1.3$ $\mu \mathrm{mol} / \mathrm{kg}$ Gd-DTPA. Mice were sacrificed after the scan-procedures were completed (after approximately 2 hours of circulation) by dissection of the diaphragm and cardiac perfusion with $5 \mathrm{~mL}$ of HBSS in order to remove blood from the vasculature via atrial- 
puncture. Then the carotid arteries were carefully excised; collars, connective tissue, and adipose tissue were removed.

MRI: Mice were placed in an animal holder and scanned in a 7T Bruker Biospec (Bruker Biospin, GmbH, Ettlingen, Germany), while under isoflurane anesthesia and warmed by a heating pad. Respiration and temperature were continuously monitored. Carotid images were obtained using a $35 \mathrm{~mm}$ diameter quadrature coil (Bruker Biospin $\mathrm{GmbH}$, Ettlingen, Germany). First, scout images were acquired in order to plan the transverse slices perpendicular to the carotids. Next, RARE $T_{1}$-weighted pulse sequences were performed with the following parameters: TR, $1014 \mathrm{~ms}$; TE, $8.2 \mathrm{~ms}$; field-of-view, 30×30 $\mathrm{mm}^{2}$; matrix size, 312×312; slice thickness, $0.5 \mathrm{~mm}$; number of averages, 32; rare partitions, 4. The obtained in-plane resolution was $96 \times 96 \mu \mathrm{m}^{2}$. Pre- and post contrast scans were made with the same gain settings. Post-injection scans were started 10 minutes after injection. Regions of interest (ROIs) were drawn on the vascular wall on the first transverse slice proximal to the collar independently by two observers. Absolute enhancement $\left(\mathrm{SI}_{\mathrm{enh}}\right)$ was calculated by dividing mean MR signal intensities of the ROI on the post-injection image by the mean MR signal intensities of the ROI on the preinjection image. Measurements of two independent observers were averaged. SPSS 15.0 (SPSS Inc) was used to determine statistical signal enhancement by testing if $\mathrm{SI}_{\mathrm{enh}} \neq$ 1 with a two-way, one-sample t-test. $\mathrm{P}<0.05$ was considered statistically significant.

Tissue preparation for TPLSM imaging: In the biodistribution study, samples of nonfixed, fresh tissue were embedded in $2 \%$ agarose gel, and covered by HBSS. For the imaging of Qdot uptake in plaques, fresh carotid arteries were imaged in either of three different ways (because plaque tissue can only be partially imaged due to high light scattering by fat tissue): 1) mounted into a home-built perfusion chamber ${ }^{40}$ (IDEE BV, Maastricht, the Netherlands) in HBSS, while under a physiological transmural pressure of $80 \mathrm{mmHg}$ (using a modified Big Ben sphygmomanometer, Riester, Germany) and viewed from the adventitial side; 2 ) dissected longitudinally, and viewed from the luminal side ('en face'); 3) dissected transversally so that the cross-section of the artery could be imaged.

To stain cell nuclei in the vascular wall, arteries were incubated in $2 \mu \mathrm{M}$ syto44 (Invitrogen, Eugene, OR/USA). Nile Red (Invitrogen, Eugene, OR/USA) was used to stain lipids. 


\section{Chapter 5}

Nile Red was dissolved in HBSS at $500 \mu \mathrm{g} / \mathrm{mL}$, containing 1\% DMSO and Pleuronic, and filtered using a $0.45 \mu \mathrm{m}$ filter. For proper labeling, arteries were incubated with either dye for at least $30^{\prime}$. For ex vivo labeling with CNA-streptavidin-Alexa568, one artery was incubated for $30^{\prime}$ in $500 \mu \mathrm{l}$ of 320 nM streptavidin-Alexa568 (Invitrogen, Eugene, OR/USA ) with bound biotinylated CNA in HBSS.

Two-photon laser scanning microscopy: A standard Bio-Rad 2100MP (Hemel Hampstead, UK) was used in TPLSM mode. The excitation source was a Spectra Physics Tsunami Ti:Sapphire laser (Mountain View, CA/USA), tuned and mode-locked at $800 \mathrm{~nm}$. The Tsunami laser produces light pulses of about $100 \mathrm{fs}$ width at a repetition rate of 82 MHz. Laser light reached the sample through a Nikon water immersion lens (60x water dipping, numerical aperture (NA) 1.0, working distance $2.8 \mathrm{~mm}$ ), incorporated in an upright Nikon E600FN microscope (Tokyo, Japan). Behind the objective, laser pulse width is estimated to be around $300 \mathrm{fs}$, based on optical properties as given by the microscope manufacturer. Three photomultipliers detected the fluorescence. Each photomultiplier accepted a different, tunable wavelength region. Initially, these filter settings were used: blue (for autofluorescence up to $500 \mathrm{~nm}$; for syto $44,420-470 \mathrm{~nm}$ ); green (for autofluorescence 500-560 nm; for Qdot525 510-540 nm); and red (for autofluorescence and Nile Red $560 \mathrm{~nm}$ and higher; for Qdot585, 570-600 nm). All three channels were optimized individually, such that they showed maximal intensities while avoiding saturation and leak-through to other channels. The three obtained images were combined into a single image. Only combined images are shown. No additional image processing was performed. An imaging speed of $0.1 \mathrm{~Hz}$ with a pixel dwell time of 37.8 us was used, and a Kalman filter over 1 frame was applied; or an imaging speed of $0.14 \mathrm{~Hz}$, pixel dwell time $10.5 \mu$ s in combination of a Kalman filter over 2 frames.

\section{Results}

The biodistribution of Qdots at 90min after intravenous injection was examined by ex vivo TPLSM. Anx- and CNA-Qdots localized mainly around liver and spleen vasculature, presumably inside vesicles in macrophages in the vicinity of the vasculature (figure 2). No kidney clearance was found, presumably due to the relatively large size of the Qdot (diameter $\approx 10 \mathrm{~nm}^{23}$ ). These results confirmed previous reports ${ }^{25,26,32,35}$. Though the 


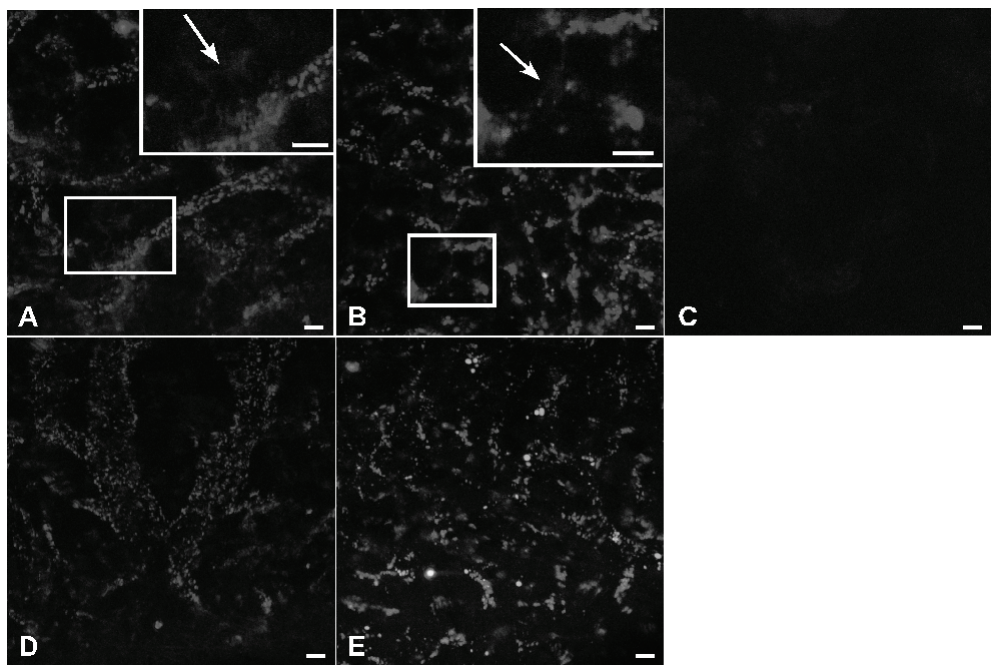

Figure 2 (below). Distribution of CNA-Qdots in A) spleen, inset is magnification of box; B) liver, inset is magnification of box; C) kidney glomerulus. Distribution of Anx-Qdots in D) spleen and E) liver. Qdots (red), autofluorescence (blue). Arrows in A) and B) indicate collagen-binding Qdots. Bar $=10 \mu \mathrm{m}$. Color figure on page 185 .

biodistributions of CNA- and Anx-Qdots were largely similar, some subtle differences became apparent. Anx-Qdots were only observed within spots; CNA-Qdots also appeared to bind collagen in vascular walls in spleen and liver (indicated by the arrows in figure 2). This is more clearly visible in figure 3, showing spleen and liver of a mouse injected with both CNA-Qdots (red) and bare streptavidin-Qdots (green). Though Qdots are not cleared by the kidneys, CNA-Qdots were observed within the glomerulus (figure 2C). CNA-, Anx-, and control-Qdots were never observed in kidney tubuli, confirming that Qdots were not renally excreted. Lung tissue showed some aggregates of Qdots, while intestine and heart tissue only sporadically showed small aggregates within microvessels (not shown).

Circulation half-lives reported in literature are highly variable, depending on, among others, size and surface chemistry ${ }^{41}$. Half-life values varying between $4.6 \mathrm{~min}$ and $19.8 \mathrm{~h}$ $26,28,32,42-44$ have been reported. The clearance of Anx- and CNA-Qdots followed a monoexponential clearance curve with circulation half-lives $\left(t_{1 / 2}\right)$ of $3.5 \mathrm{~min}$ for CNA-Qdots and 5.3min for Anx-Qdots (figure 4). To determine the component of the bimodal Qdot that causes fast clearance, also circulation half-lives of bare streptavidin-Qdots (without ligand or Gd-wedge) and Qtracker (Qdot without streptavidin) were determined. Bare 

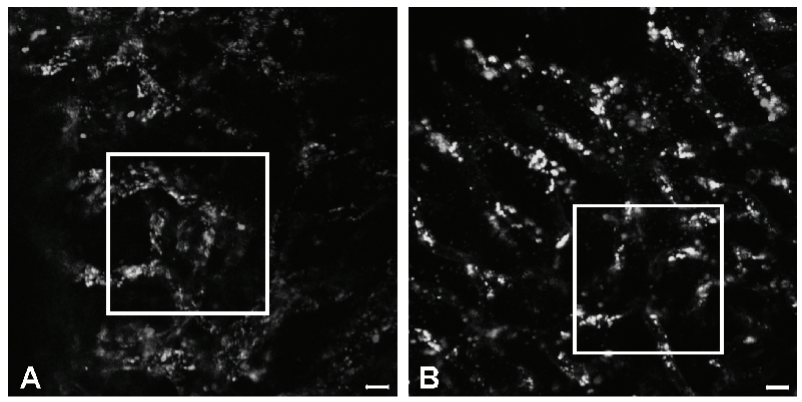

Figure 3. Difference in distribution of bare Qdots (green) and CNA-Qdots (red). A) spleen, B) liver, C) magnification of boxed part in A), D) magnification of boxed part in B). Arrows indicate collagen-specific binding of CNA-Qdots. Bar $=10 \mu \mathrm{m}$. Color figure on page 185 .
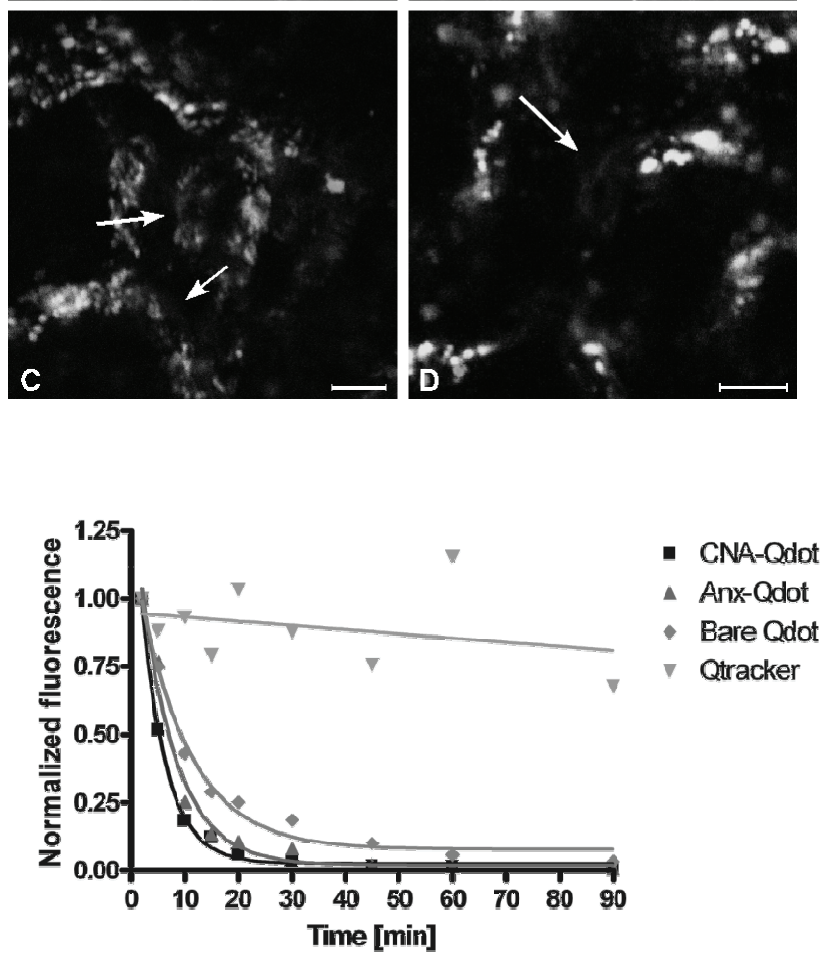

Figure 4. Clearance of CNA-Qdots, Anx-Qdots, bare Qdots (without ligand and Gd-wedge, but with streptavidin), and Qtracker (Qdot without streptavidin). Data points are normalized to fluorescence intensity at $\mathrm{t}=$ $2 \mathrm{~min}$ (see the Materials and Methods section). Mono-exponential curves are fitted through the data. The fit for Qtracker is indicative due to scattering of data points.

streptavidin-Qdots have a half-life of $6.6 \mathrm{~min}$, while the half-life of Qtracker could not be determined due to high variation in data points. However, it is clear from figure 4 that this half-life is much greater (> 90min) than for any of the streptavidin-coated Qdots. Consequently, the presence of streptavidin is most likely responsible for the fast clearance of the bimodal Qdots. 


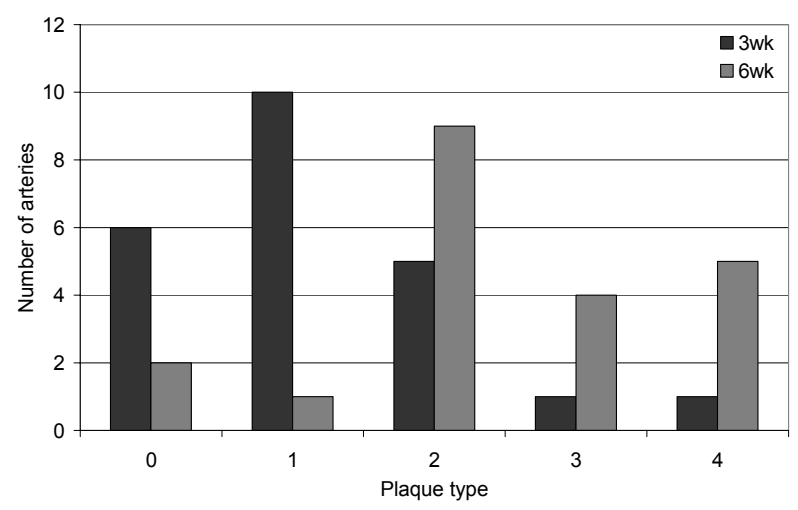

Figure 5. Plaque types in carotid arteries for three-week and six-week collar-bearing ApoE -/- mice. Plaque types of both carotid arteries of all mice are combined. Types were binned as follows: 0 , no plaque; 1 , small, not occlusive; 2 , moderate, up to $50 \%$ occlusive in lumen diameter; 3 , large, $50-70 \%$ occlusive in lumen diameter; 4 , severe, $70-100 \%$ occlusive in lumen diameter.

The size of the collar-induced plaques in ApoE -/- mice was determined based on lightmicroscopic evaluation after excision of the carotids. Plaques were assessed as one of the following types: 0 , no plaque; 1 , small, non-occlusive plaque; 2 , moderate plaque, up to $50 \%$ occlusion of lumen diameter; 3 , large plaque, $50-70 \%$ occlusion of lumen diameter; and 4 , severe plaque, more than $70 \%$ occlusion of lumen diameter. The median for three-week collar plaques was type 1, while for six-week collar plaques the median was of type 2 (figure 5). Type 1 plaques consisted mainly of a collection of small lipid cores. With increasing plaque size, the size of the lipid cores increased while the number of lipid cores decreased. Type 4 plaques consist generally of few but very large lipid cores.

Arteries were imaged ex vivo with TPLSM. Plaque tissue is difficult to visualize due to limited penetration depth of light in plaque (fat) tissue. With the set-up used, plaques could be imaged up to a thickness of approximately $100 \mu \mathrm{m}^{20}$. Depending on the viewangle ('en face', transversal, or mounted arteries, see Materials and Methods), various aspects of the plaque could be visualized. When imaging mounted arteries, generally less Qdots were observed. This can be explained by the fact that Qdots were localized 
Table 1. Overview of CNA-, Anx-, and control-Qdot labeling in plaque-containing carotid arteries.

\begin{tabular}{|c|c|c|c|}
\hline ligand & $\begin{array}{l}\text { plaque } \\
\text { type }\end{array}$ & $\begin{array}{l}\text { no. of } \\
\text { arteries }\end{array}$ & Qdot labeling of plaque \\
\hline \multirow[t]{5}{*}{ Control } & 0 & 2 & In one artery minor endothelial labeling, in one artery no labeling. \\
\hline & 1 & 3 & $\begin{array}{l}\text { In one artery minor endothelial labeling, two arteries showed no label- } \\
\text { ing. }\end{array}$ \\
\hline & 2 & 8 & $\begin{array}{l}\text { In seven arteries minor to moderate endothelial labeling (figure } \\
6 A / B / C \text { ), in one artery no labeling. }\end{array}$ \\
\hline & 3 & 1 & Minor endothelial labeling in one artery. \\
\hline & 4 & 1 & Moderate endothelial labeling in one artery (figure 6D). \\
\hline \multirow[t]{5}{*}{ CNA } & 0 & 3 & Minor Qdot labeling of fibrous caps in three arteries. \\
\hline & 1 & 5 & $\begin{array}{l}\text { Minor to moderate Qdot labeling of fibrous cap around lipid pools in all } \\
\text { five arteries (figure 7A). }\end{array}$ \\
\hline & 2 & 1 & The artery shows weak labeling of fibrous cap. \\
\hline & 3 & 3 & $\begin{array}{l}\text { In one carotid artery strong labeling of fibrous cap (figure } 8 \mathrm{~A} / \mathrm{B} \text { ), a } \\
\text { second artery shows CNA-Qdots on fibrous cap covering lipid pools } \\
\text { (figure } 8 \mathrm{C} \text { ); the third artery shows weak labeling. Figures } 8 \mathrm{~A} / \mathrm{B} / \mathrm{D} / \mathrm{F} \\
\text { show the same artery. }\end{array}$ \\
\hline & 4 & 2 & $\begin{array}{l}\text { In one artery strong labeling inside lipid pool (figure 7B), in other artery } \\
\text { strong labeling of basal lamina around lipid pools (figure 8E) }\end{array}$ \\
\hline \multirow[t]{5}{*}{ Anx } & 0 & 3 & Minor endothelial labeling in three arteries. \\
\hline & 1 & 3 & $\begin{array}{l}\text { Some endothelial labeling in two arteries that also show minor uptake } \\
\text { of Qdots in lipid pools (figure } 10 \mathrm{C} / \mathrm{D} \text { ). One artery shows no labeling. }\end{array}$ \\
\hline & 2 & 5 & $\begin{array}{l}\text { Minor to moderate endothelial labeling in four arteries. Minor labeling } \\
\text { inside lipid pool in another artery (figure 11C) }\end{array}$ \\
\hline & 3 & 1 & $\begin{array}{l}\text { This artery showed endothelial labeling, but also labeling inside lipid } \\
\text { pools (figure 10A/B, same artery). Stronger labeling than in types 1-2. }\end{array}$ \\
\hline & 4 & 3 & $\begin{array}{l}\text { Two arteries were completely occluded (collaterals had formed), no } \\
\text { Qdot labeling. One artery showed strong labeling inside lipid pool (fig- } \\
\text { ure } 11 B / D \text { ), and also endothelial labeling (figure } 11 \mathrm{~A}, 11 \mathrm{~A} / \mathrm{B} / \mathrm{D} \text { are the } \\
\text { same artery). }\end{array}$ \\
\hline
\end{tabular}

on or just underneath the endothelium, and excitation as well as emission light had to go through plaque tissue in order to reach the Qdots. In general, this means that only plaque shoulders could be imaged properly. However, an advantage of mounted arteries is that they are in a near-physiological situation as they are pressurized at normal transmural pressures. For an overview of the entire plaque, the artery was best imaged 


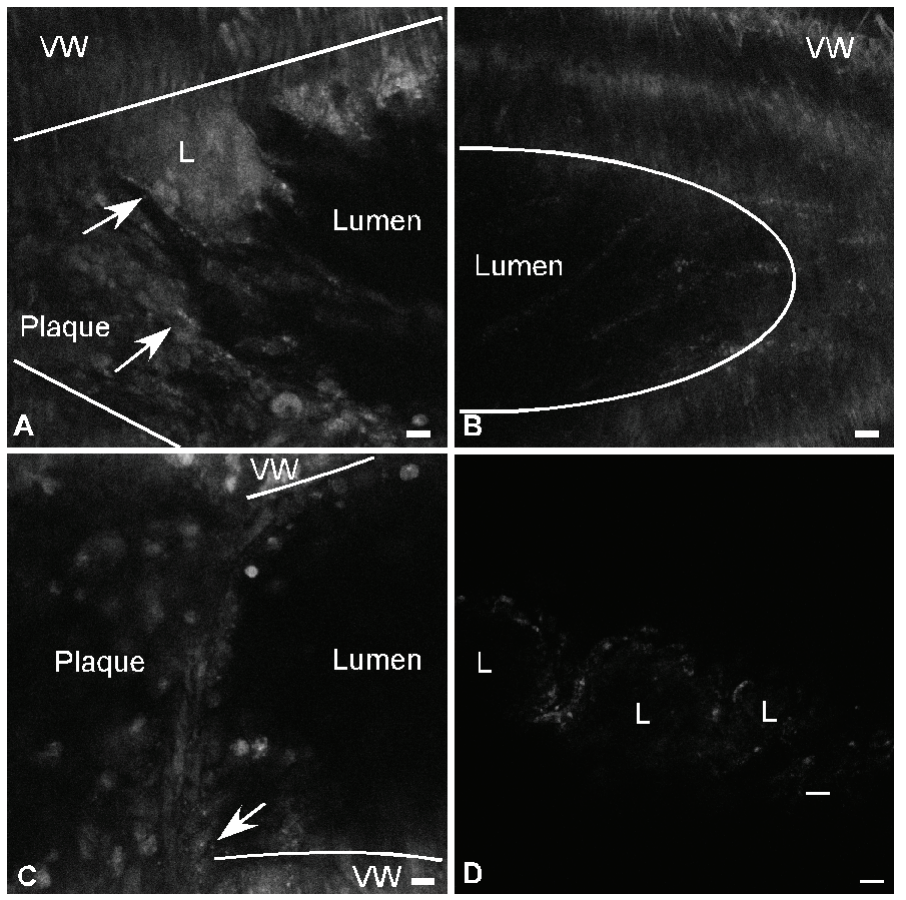

Figure 6. A and B) Control-Qdots (green) in three-week collar plaques. Both images show carotid arteries in a flow chamber and under $80 \mathrm{mmHg}$ transmural pressure. A) Control-Qdots are bound to endothelium covering lipid pools (type 2). B) Control-Qdots are seen in the grooves of elastic laminae in an area adjacent to plaque (type 2). C and D) Control-Qdots (green) in six-week collar plaques. C) Mounted carotid artery under $80 \mathrm{mmHg}$ (type 2). Endothelial cells on plaque are labeled with control-Qdots, indicated by arrow. D) 'En face' view of carotid artery (type 4). White lines indicate border between media and intima layers. Arrows indicate controlQdots bound to endothelium. Blue in A) and C) are nucleic acids, and in B) and D) autofluorescence. Red is lipid. ' $L$ ' is lipid pool, 'VW' is vascular wall. Bar $=10 \mu \mathrm{m}$. Color figure on page 186.

'en face'; the luminal surface of lipid pools could then be visualized. Finally, crosssections were best suitable to visualize the inside of a plaque or lipid pool.

Each of three views revealed different parts of the plaque, and they were used in this study alternately to gather as much information on the Qdot intra-plaque distribution as possible. A quantitative analysis of plaque labeling is impossible using TPLSM images, as the plaque cannot be imaged entirely with either of the view angles. Therefore, a semi-quantitative and qualitative description of our findings is presented here. An overview of the results of 3 and 6 week old collar-bearing ApoE -/- mice is given in table 1 and is further discussed in the following paragraphs. 

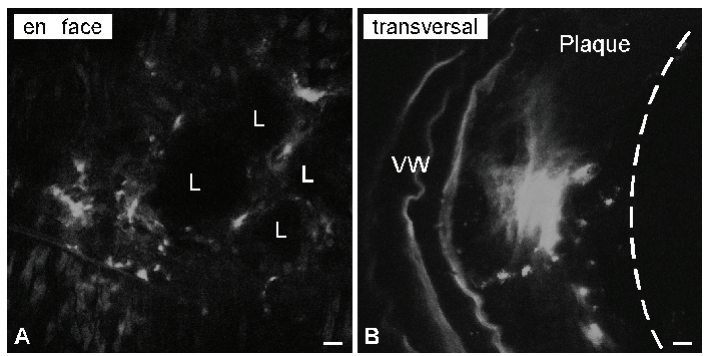

Figure 7. CNA-Qdots (green) in three-week collar plaques. A) 'En face' view (type 1). B) Transverse view (type 4). Dashed line indicates border between plaque and lumen. In A) blue are nucleic acids, in B) autofluorescence. $B$ ) shows the sum of a small stack of images. ' $\mathrm{L}$ ' is lipid core, ' $V W$ ' is vascular wall. $\mathrm{Bar}=10 \mu \mathrm{m}$. Color figure on page 186.

Three- and six-week plaques showed minor staining with control-Qdots. If labeling was observed, it localized to endothelium, and mostly plaque-endothelium (figure 6A/C/D). In smaller plaques, the grooves of the elastic laminae are visible due to the endothelial staining (figure 6B).

In three-week plaques, CNA-Qdots were mainly found directly underneath the endothelial layer, i.e. on the fibrous caps surrounding lipid cores for all plaque types. When visualized 'en face', lipid cores were visible by their fibrous caps containing collagenbound CNA-Qdots, indicating that the lipids were embedded in fibrous caps (figure 7A). On one occasion, CNA-Qdots were also clearly visible inside a type 4 plaque (figure 7B). On another occasion, CNA-Qdots also bound to the basal lamina of a non-plaque containing carotid artery (not shown).

In six-week collar-carotids, plaques were generally larger in size as compared to plaques of three-week collar carotids (see figure 5). Larger plaques contained more CNA-Qdots. Collagen-networks were visualized at the shoulder of plaques (fibrous caps, figure $8 A / B)$. When viewed 'en face', collagen-networks were visualized just underneath the plaque-endothelium around (figure 8C) and in between lipid-cores (figure 8D/E).

It appeared that only specific spots in the plaque were labeled with CNA-Qdots (hotspots, figures $8 \mathrm{D} / 8 \mathrm{~F} / 7 \mathrm{~B})$. This is in contrast to the labeling behavior of the much smaller CNA-OG488, which labels collagen in the entire plaque ${ }^{19}$. In order to verify that CNAQdot indeed labels specific sites of collagen and to exclude that the cause of this difference is a different collagen content, an atherosclerotic artery labeled in vivo with CNAQdots (green) was subsequently labeled ex vivo with CNA-streptavidin-Alexa568 (red, figure 9). Ex vivo labeling generally results in more labeling than in vivo labeling. Fur- 


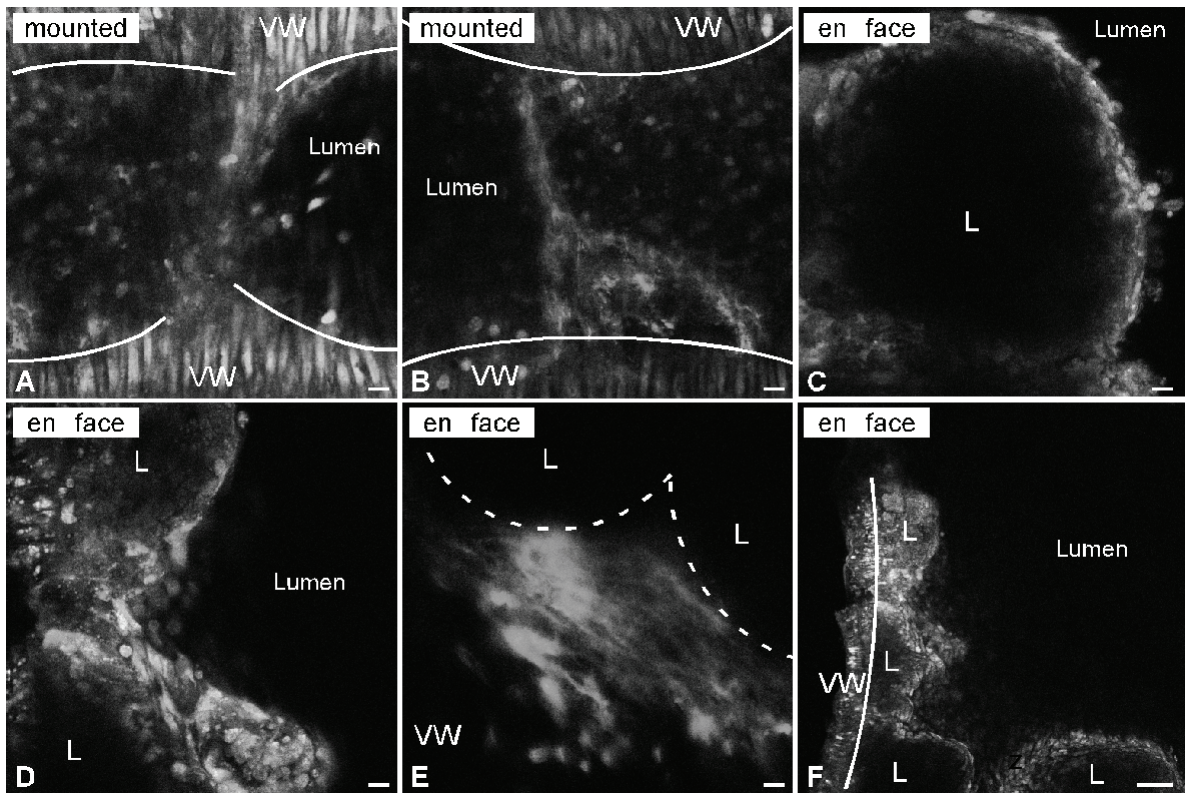

Figure 8. CNA-Qdots (green) in six-week collar plaques. A) and B) Mounted artery under $80 \mathrm{mmHg}$ transmural pressure, showing fibrous caps labeled with CNA-Qdots (same artery, type 3). C) 'En face' view, showing a lipid pool with CNA-Qdots just underneath the endothelium (type 3). D) 'En face' view, collagen network visible in between two lipid pools (type 3). E) 'En face' view, collagen network visible in between two lipid pools (type 4). F) 'En face' view showing multiple lipid pools, but only one (same as in D) shows bound CNA-Qdots. Blue are nucleic acids, red is lipid. White lines indicate border between media and intima, dotted lines in E) indicate border of lipid pools. ' $\mathrm{L}$ ' is lipid pool, ' $V W$ ' is vascular wall. Bar $=10 \mu \mathrm{m}$, except in F), bar $=50 \mu \mathrm{m}$. Color figure on page 187.

thermore, since CNA-streptavidin-Alexa568 is much smaller than CNA-Qdot, CNAstreptavidin-Alexa568 labels more collagen present. High amounts of broadly distributed collagen are present in these lesions (figure 9). This suggests that CNA-Qdots indeed preferentially label accessible hot-spots of collagen in a plaque.

Anx-Qdots localized to endothelium (figure 10A/B, white arrows) and inside lipid cores of three-week plaques. Inside lipid cores, Anx was visible in vesicle-shaped structures (figure $10 C / D$, orange arrows), or seemed attached to cells inside the cores, possibly macrophages (figure 10A/D, yellow arrows).

Anx-Qdots bound to endothelium of six-week plaques (figure 11A). In addition, AnxQdots were found inside lipid cores, on one occasion showing very strong labeling (figure 11B). Anx-Qdots also bound to (foam) cells (figure 11C/D) inside lipid pools. As with 


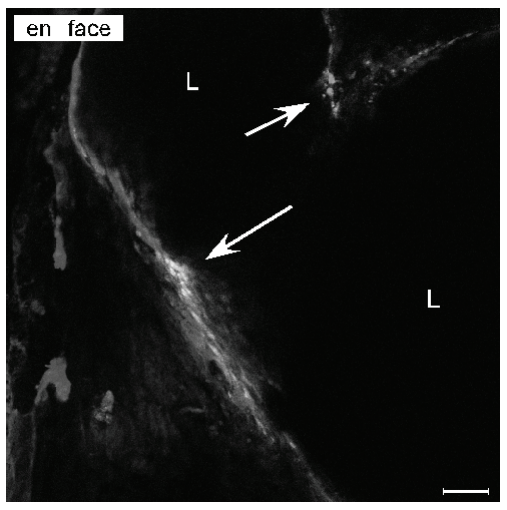

Figure 9. Two-photon image showing 'en face' view of a plaque, in vivo injected with CNA-Qdot (green) and ex vivo labeled with CNA-streptavidin-Alexa568 (red). Ex vivo labeling resulted in abundant collagen labeling, revealing that CNAQdots indeed only label accessible hot-spots of collagen within the plaque. Blue is syto44. Bar $=50 \mu \mathrm{m}$. Color figure on page 187.

CNA-Qdots, Anx-Qdot penetration into the plaque is restricted and occurs only at hotspots.

In vivo MRI was also performed. To quantify the signal intensity in the plaque, regions of interest (ROIs) were drawn independently by two observers on a transverse slice of a $\mathrm{T}_{1}$-weighted pulse sequence, on the plaque-portion of the carotid artery. Three- and sixweek collar mice combined, CNA-Qdots lead on average to a minor, but significant signal intensity increase (post-scan compared to pre-scan) of $6.2 \% \pm 2.1 \%$ (mean \pm SEM), while Anx-Qdots as well as control-Qdots lead to a non-significant average decrease of $2.3 \% \pm 1.4 \%$ and $0.23 \% \pm 2.2 \%$, respectively. This coincides with TPLSM results, as there appears to be more labeling in the plaques labeled with CNA-Qdots than those labeled with Anx-Qdots (see table 1 and figures 7/8/10/11). The fact that these changes in signal intensity are small, despite the high longitudinal relaxivity $\left(3000-4500 \mathrm{mM}^{-1} \mathrm{~s}^{-1}\right.$ per Qdot) reported previously ${ }^{23}$, can be explained by the low total injected dose of GdDTPA $(1.3 \mu \mathrm{mol} / \mathrm{kg})$. Furthermore, as can be seen in the TPLSM images, CNA- and AnxQdots were present only in hot-spots in the plaque, and not in the entire vascular wall. This is unfavorable for visualization with MRI as this imaging technique is relatively insensitive to contrast agents. This means that a high pay-load of contrast agents within the plaque is required for MRI visualization. In the used experimental set-up, higher dosing is limited by low stock concentration of Qdots. Similar bimodal Qdots, functionalized with angiogenic ligand cNGR, have been used successfully in the MRI visualization of tumor tissue using a low dose ${ }^{33}$. 

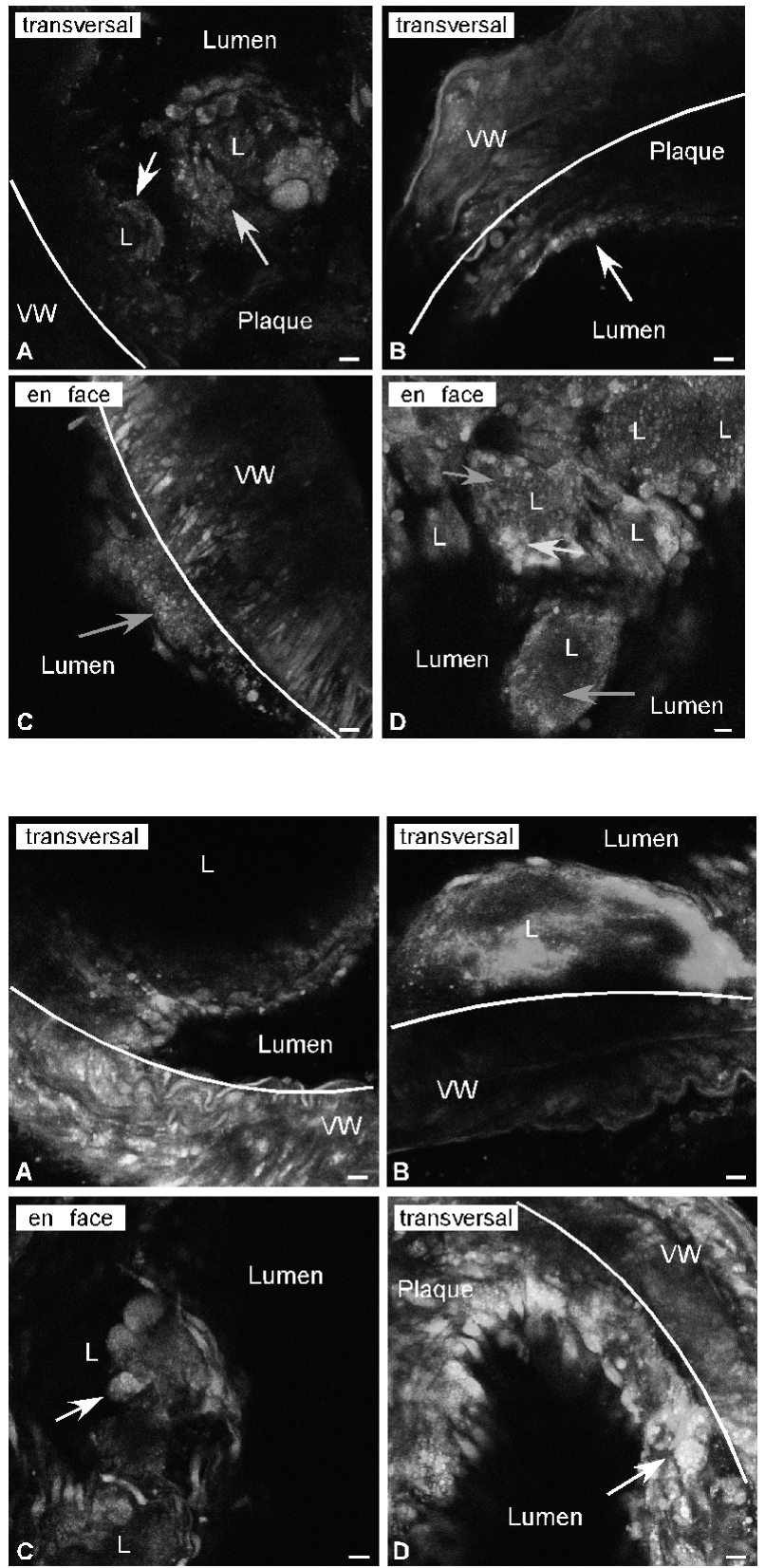

Figure 10. Anx-Qdots (green) in three-week collar plaques. A) Transverse view of plaque (type 3). B) Transverse view of plaque (type 3). C) 'En face' view of plaque (type 1). Vascular wall is visible because the cutting surface curled up. D) 'En face' view of plaque (type 1). White lines indicate border between media and intima. White arrows indicate endothelial labeling by AnxQdots, yellow arrows indicate cellular Anx-Qdots, and orange arrows indicate vesicular AnxQdots. ' $\mathrm{L}$ ' indicates lipid pool, ' $V W$ ' indicates vascular wall. Red/orange is lipid; blue are nucleic acids. Bar $=10 \mu \mathrm{m}$. Color figure on page 188 .

Figure 11. Anx-Qdots (green) in six-week collar plaques. A) Transverse slice of carotid artery. AnxQdots are visible on the endothelium covering the lipid pool (type 4). B) Transverse slice of carotid artery, showing a high load of Anx-Qdots inside a lipid pool (type 4). C) 'En face' view of carotid artery (type 2). Anx-Qdots are visible in a foam cell within a lipid pool. D) Transverse view of plaque in carotid artery (type 4). Arrows indicate (foam) cell that had taken up Anx-Qdots. White lines indicate border between media and intima. ' $\mathrm{L}$ ' is lipid pool; ' $V W$ ' is vascular wall. Blue are nucleic acids, red is lipid. Bar $=10$ $\mu \mathrm{m}$. Color figure on page 188. 

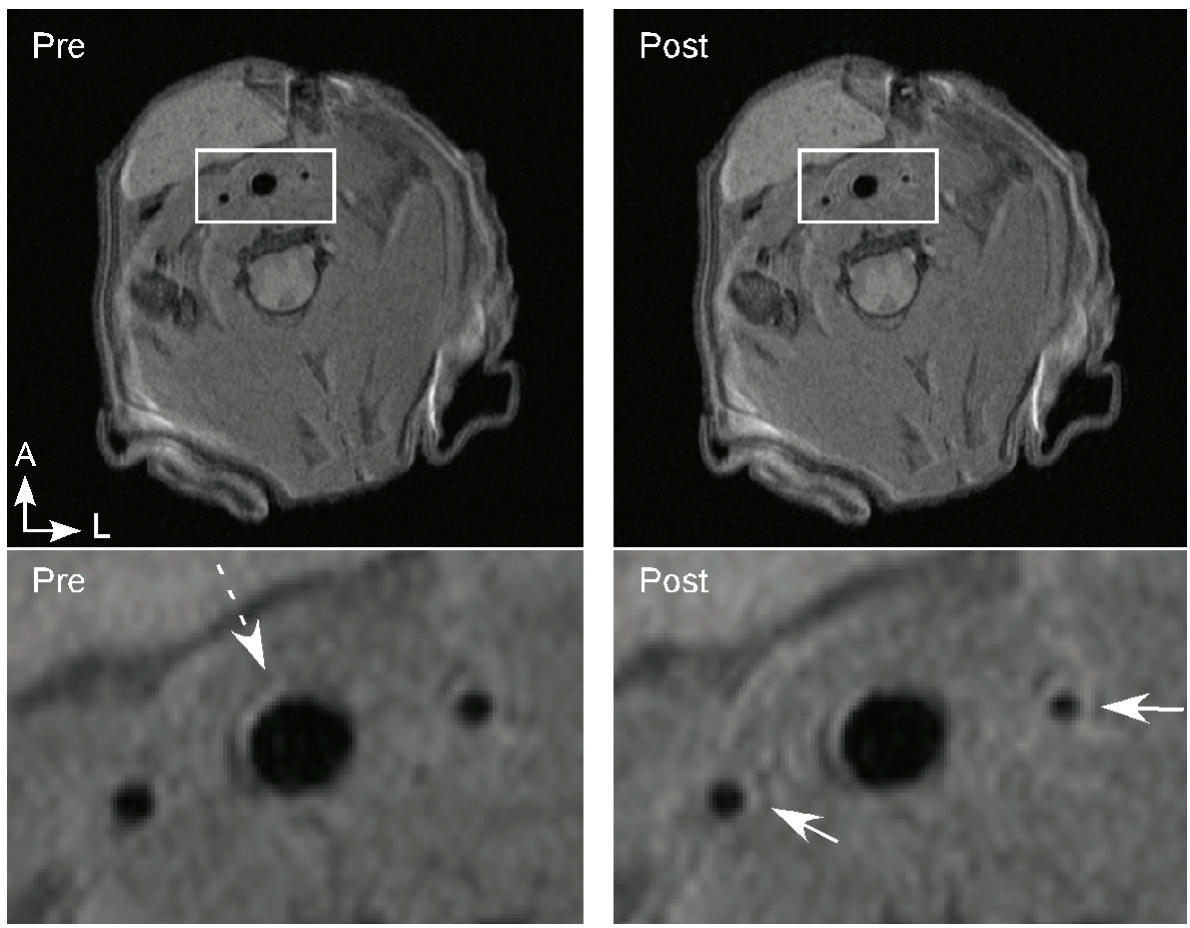

Figure 12. Pre- and post-MR images of first transverse slice proximal to collar. This is a 6-week collar mouse, intravenously injected with CNA-Qdots. Bottom row shows magnification of boxed parts on top row. ' $A$ ' indicates anterior, ' $L$ ' indicates left. White (non-dashed) arrows indicate signal intensity increases in vascular walls of carotid arteries. Dashed arrow indicates trachea. Left carotid artery is the same artery as in figure 8C.

\section{Discussion}

Qdots have to cross several barriers in order to reach the inside of a plaque. The glycocalyx is the first barrier, a matrix consisting of proteoglycans and glycoproteins attached to and covering the endothelium. It is hypothesized that this layer is perturbed at sites of atherosclerosis ${ }^{45}$. The next barrier is the endothelium. Endothelial permeability is based on two processes: transcytosis through the endothelial cells (active vesicle transport), and paracellular transport through gaps between the endothelial cells (passive diffusion) ${ }^{46}$. Healthy endothelium allows solutes up to $2 \mathrm{~nm}$ in diameter ${ }^{47}$ to pass via these intercellular gaps. Larger structures may pass the endothelium by transcytosis. As a result of endothelial activation, both transcytosis is increased and endothelial gaps become larger, which is known to occur at sites of inflammation, such as in atheroscle- 
rotic plaques ${ }^{48}$. Increased endothelial permeability is induced by, e.g., reactive oxygen species ${ }^{49}$ and cytokines ${ }^{50}$ released by inflammatory cells. The third barrier is the fibrous cap, an extracellular matrix consisting of collagen and elastin. Although the synthesis of extracellular matrix components is upregulated in plaques, also matrixdegrading matrix metalloproteinases that are released by, among others, macrophages are active ${ }^{1}$, presumably resulting in less organized extracellular matrix. Qdots are indeed able to pass through the intimal layer, but only at specific sites in the plaque, as shown in figures 7,10 , and 11 , most likely through perturbed endothelium.

Hot-spot labeling by CNA-Qdot (figures 7/8) contrasts a previous study using CNAOG488 to visualize atherosclerotic plaques in old ApoE -/- mice ${ }^{19}$. CNA-OG488, a fluorescent polypeptide, is substantially smaller than CNA-Qdot $(\approx 0.5 \mathrm{~nm}$ and $10 \mathrm{~nm}$ in diameter, respectively). CNA-OG488 labeled fibrous caps in the entire plaque and even the tunica intima adjacent to plaques ${ }^{19}$. Even though in the CNA-OG488 study ten-fold more CNA was injected and a different animal model was used, hot-spots of CNA-Qdots in plaques strongly suggest that CNA-Qdots do bind at specific sites within the plaque. Based on the assumption that CNA-Qdots $(\approx 10 \mathrm{~nm})$ enter the plaque through sufficiently large gaps in endothelium, this may also be assumed for Anx-Qdots. Indeed, Anx-Qdots were only seen inside few lipid pools, though this may also result from PSexpressing cells or debris present only in these lipid pools. On the other hand, ${ }^{99 \mathrm{~m}} \mathrm{Tc}$ Annexin, of similar size as CNA-OG488, has been used to image atherosclerotic plaques in ApoE -/- aortas. ${ }^{99 \mathrm{~m}} \mathrm{Tc}$-Annexin was indeed able to label large areas of aortic plaques, suggesting that ${ }^{99 \mathrm{~m}} \mathrm{Tc}$-Annexin does not label plaque tissue size-selectively ${ }^{12}$.

Hot-spot labeling by Qdots indicates increased intimal permeability and/ or disruption, and increased inflammation at these sites. It is intriguing to speculate that sites where Qdots penetrate the plaque, also platelets and plasma proteins, such as coagulation factors, extravasate into the plaque. Hence, the presence of Qdots at sites inside a plaque may indicate where inflammation is increased and rupture-prone sites are located. Whether these sites indeed indicate rupture-prone sites remains to be elucidated. CNA- and Anx-Qdots provide a promising novel approach of vulnerable plaque imaging, as it is based on size-selection, complementary to molecular targeting. 
Chapter 5

\section{Acknowledgements}

We thank Maarten Merkx for providing biotinylated CNA, and Rik Tinnemans for biotechnical assistance. This study was supported by SenterNovem, BSIK grant 03033. TPLSM was financed by The Netherlands Organization for Scientific Research, NWO 902-16-276. 


\section{References}

J. O. Deguchi, M. Aikawa, C. H. Tung, E. Aikawa, D. E. Kim, V. Ntziachristos, R. Weissleder and

P. Libby; "Inflammation in atherosclerosis: visualizing matrix metalloproteinase action in macrophages in vivo", Circulation 2006, 114(1) 55-62.

D. Hartung, M. Schafers, S. Fujimoto, B. Levkau, N. Narula, K. Kopka, R. Virmani, C. Reutelingsperger, L. Hofstra, F. D. Kolodgie, A. Petrov and J. Narula; "Targeting of matrix metalloproteinase activation for noninvasive detection of vulnerable atherosclerotic lesions", Eur J Nucl Med Mol Imaging 2007,

M. Schafers, B. Riemann, K. Kopka, H.-J. Breyholz, S. Wagner, K. P. Schafers, M. P. Law, O. Schober and B. Levkau; "Scintigraphic Imaging of Matrix Metalloproteinase Activity in the Arterial Wall In vivo", Circulation 2004, 109(21) 2554-2559.

V. Amirbekian, M. J. Lipinski, K. C. Briley-Saebo, S. Amirbekian, J. G. Aguinaldo, D. B. Weinreb, E. Vucic, J. C. Frias, F. Hyafil, V. Mani, E. A. Fisher and Z. A. Fayad; "Detecting and assessing macrophages in vivo to evaluate atherosclerosis noninvasively using molecular MRI", Proc Natl Acad Sci U S A 2007, 104(3) 961-6.

A. Tawakol, R. Q. Migrino, G. G. Bashian, S. Bedri, D. Vermylen, R. C. Cury, D. Yates, G. M. LaMuraglia, K. Furie, S. Houser, H. Gewirtz, J. E. Muller, T. J. Brady and A. J. Fischman; "In vivo 18F-fluorodeoxyglucose positron emission tomography imaging provides a noninvasive measure of carotid plaque inflammation in patients", J Am Coll Cardiol 2006, 48(9) 1818-24.

Z. Zhang, J. Machac, G. Helft, S. G. Worthley, C. Tang, A. G. Zaman, O. J. Rodriguez, M. S. Buchsbaum, V. Fuster and J. J. Badimon; "Non-invasive imaging of atherosclerotic plaque macrophage in a rabbit model with F-18 FDG PET: a histopathological correlation", BMC Nucl Med 2006, 6(3.

E. Spuentrup, A. Buecker, M. Katoh, A. J. Wiethoff, E. C. Parsons, Jr., R. M. Botnar, R. M. Weisskoff, P. B. Graham, W. J. Manning and R. W. Gunther; "Molecular magnetic resonance imaging of coronary thrombosis and pulmonary emboli with a novel fibrin-targeted contrast agent", Circulation 2005, 111(11) 1377-82.

R. J. J. Miserus, L. Prinzen, M. V. Herias, T. M. Hackeng, A. Dirksen, W. Adriaens, M. A. M. J. Van Zandvoort, M. B. Lobbes, M. J. Daemen, J. J. Van Engelshoven, S. Heeneman and M. E. Kooi; "In vivo MR Imaging of Acute Thrombus Formation Using a Bimodal Contrast Agent", Proc Intl Soc Mag Reson Med 2008, 16(22. 


\section{Chapter 5}

9

C. P. Reutelingsperger and W. L. van Heerde; "Annexin V, the regulator of phosphatidylserinecatalyzed inflammation and coagulation during apoptosis", Cell Mol Life Sci 1997, 53(6) 52732.

J. F. Tait and D. Gibson; "Phospholipid binding of annexin V: effects of calcium and membrane phosphatidylserine content", Arch Biochem Biophys 1992, 298(1) 187-91.

F. D. Kolodgie, J. Narula, A. P. Burke, N. Haider, A. Farb, Y. Hui-Liang, J. Smialek and R. Virmani; "Localization of apoptotic macrophages at the site of plaque rupture in sudden coronary death", Am J Pathol 2000, 157(4) 1259-68.

2

S. Isobe, S. Tsimikas, J. Zhou, S. Fujimoto, M. Sarai, M. J. Branks, A. Fujimoto, L. Hofstra, C. P. Reutelingsperger, T. Murohara, R. Virmani, F. D. Kolodgie, N. Narula, A. Petrov and J. Narula; "Noninvasive imaging of atherosclerotic lesions in apolipoprotein E-deficient and low-densitylipoprotein receptor-deficient mice with annexin A5", J Nucl Med 2006, 47(9) 1497-505.

F. D. Kolodgie, A. Petrov, R. Virmani, N. Narula, J. W. Verjans, D. K. Weber, D. Hartung, N. Steinmetz, J. L. Vanderheyden, M. A. Vannan, H. K. Gold, C. P. Reutelingsperger, L. Hofstra and J. Narula; "Targeting of apoptotic macrophages and experimental atheroma with radiolabeled annexin V: a technique with potential for noninvasive imaging of vulnerable plaque", Circulation 2003, 108(25) 3134-9.

4

L. L. Johnson, L. Schofield, T. Donahay, N. Narula and J. Narula; "99mTc-annexin V imaging for in vivo detection of atherosclerotic lesions in porcine coronary arteries", J Nucl Med 2005, 46(7) 1186-93.

5 F. Rouzet, M. Dominguez Hernandez, F. Hervatin, L. Sarda-Mantel, A. Lefort, X. Duval, L. Louedec, B. Fantin, D. Le Guludec and J. B. Michel; "Technetium 99m-labeled annexin V scintigraphy of platelet activation in vegetations of experimental endocarditis", Circulation 2008, 117(6) 781-9.

6 L. Hofstra, I. H. Liem, E. A. Dumont, H. H. Boersma, W. L. van Heerde, P. A. Doevendans, E. De Muinck, H. J. Wellens, G. J. Kemerink, C. P. Reutelingsperger and G. A. Heidendal; "Visualisation of cell death in vivo in patients with acute myocardial infarction", Lancet 2000, 356(9225) 209-12.

17 B. L. Kietselaer, C. P. Reutelingsperger, G. A. Heidendal, M. J. Daemen, W. H. Mess, L. Hofstra and J. Narula; "Noninvasive detection of plaque instability with use of radiolabeled annexin A5 in patients with carotid-artery atherosclerosis", N Engl J Med 2004, 350(14) 1472-3.

Y. Lampl, M. Lorberboym, F. G. Blankenberg, M. Sadeh and R. Gilad; "Annexin V SPECT imaging of phosphatidylserine expression in patients with dementia", Neurology 2006, 66(8) 12534. 
R. T. Megens, M. G. Oude Egbrink, J. P. Cleutjens, M. J. Kuijpers, P. H. Schiffers, M. Merkx, D. W. Slaaf and M. A. van Zandvoort; "Imaging Collagen in Intact Viable Healthy and Atherosclerotic Arteries Using Fluorescently Labeled CNA35 and Two-Photon Laser Scanning Microscopy", Mol Imaging 2007, 6(4) 247-60.

R. T. A. Megens, M. G. A. o. Egbrink, M. Merkx, D. W. Slaaf and M. A. M. J. van Zandvoort; "Two-photon microscopy on vital carotid arteries: imaging the relationship between collagen and inflammatory cells in atherosclerotic plaques", Journal of Biomedical Optics 2008, 13(4) 044022-10.

H. C. Stary, A. B. Chandler, R. E. Dinsmore, V. Fuster, S. Glagov, W. Insull, Jr., M. E. Rosenfeld, C. J. Schwartz, W. D. Wagner and R. W. Wissler; "A Definition of Advanced Types of Atherosclerotic Lesions and a Histological Classification of Atherosclerosis : A Report From the Committee on Vascular Lesions of the Council on Arteriosclerosis, American Heart Association", Circulation 1995, 92(5) 1355-1374.

K. N. Krahn, C. V. Bouten, S. van Tuijl, M. A. van Zandvoort and M. Merkx; "Fluorescently labeled collagen binding proteins allow specific visualization of collagen in tissues and live cell culture", Anal Biochem 2006, 350(2) 177-85.

L. Prinzen, R. J. Miserus, A. Dirksen, T. M. Hackeng, N. Deckers, N. J. Bitsch, R. T. Megens, K. Douma, J. W. Heemskerk, M. E. Kooi, P. M. Frederik, D. W. Slaaf, M. A. Zandvoort and C. P. Reutelingsperger; "Optical and Magnetic Resonance Imaging of Cell Death and Platelet Activation Using Annexin A5-Functionalized Quantum Dots", Nano Lett 2007, 7(1) 93-100.

U. Resch-Genger, M. Grabolle, S. Cavaliere-Jaricot, R. Nitschke and T. Nann; "Quantum dots versus organic dyes as fluorescent labels", Nat Methods 2008, 5(9) 763-75.

M. E. Akerman, W. C. Chan, P. Laakkonen, S. N. Bhatia and E. Ruoslahti; "Nanocrystal targeting in vivo", Proc Natl Acad Sci U S A 2002, 99(20) 12617-21.

B. Ballou, B. C. Lagerholm, L. A. Ernst, M. P. Bruchez and A. S. Waggoner; "Noninvasive imaging of quantum dots in mice", Bioconjug Chem 2004, 15(1) 79-86.

H. Chen, Y. Wang, J. Xu, J. Ji, J. Zhang, Y. Hu and Y. Gu; "Non-invasive Near Infrared Fluorescence Imaging of CdHgTe Quantum Dots in Mouse Model", J Fluoresc 2008,

N. Y. Morgan, S. English, W. Chen, V. Chernomordik, A. Russo, P. D. Smith and A. Gandjbakhche; "Real time in vivo non-invasive optical imaging using near-infrared fluorescent quantum dots", Acad Radiol 2005, 12(3) 313-23. lecular and cellular imaging", Adv Drug Deliv Rev 2008, 60(11) 1226-40. 


\section{Chapter 5}

30

31

H. Kobayashi, Y. Hama, Y. Koyama, T. Barrett, C. A. Regino, Y. Urano and P. L. Choyke; "Simultaneous multicolor imaging of five different lymphatic basins using quantum dots", Nano Lett 2007, 7(6) 1711-6.

1 W. Cai, D. W. Shin, K. Chen, O. Gheysens, Q. Cao, S. X. Wang, S. S. Gambhir and X. Chen; "Peptide-Labeled Near-Infrared Quantum Dots for Imaging Tumor Vasculature in Living Subjects", Nano Lett 2006, 6(4) 669-676.

X. Gao, Y. Cui, R. M. Levenson, L. W. Chung and S. Nie; "In vivo cancer targeting and imaging with semiconductor quantum dots", Nat Biotechnol 2004, 22(8) 969-76.

M. Oostendorp, K. Douma, T. M. Hackeng, A. Dirksen, M. J. Post, M. A. M. J. van Zandvoort and W. H. Backes; "Quantitative Molecular Magnetic Resonance Imaging of Tumor Angiogenesis Using cNGR-Labeled Paramagnetic Quantum Dots", Cancer Res 2008, 68(18) 76767683.

4

S. Kim, Y. T. Lim, E. G. Soltesz, A. M. De Grand, J. Lee, A. Nakayama, J. A. Parker, T. Mihaljevic, R. G. Laurence, D. M. Dor, L. H. Cohn, M. G. Bawendi and J. V. Frangioni; "Near-infrared fluorescent type II quantum dots for sentinel lymph node mapping", Nat Biotechnol 2004, 22(1) 93-7.

Y. Inoue, K. Izawa, K. Yoshikawa, H. Yamada, A. Tojo and K. Ohtomo; "In vivo fluorescence imaging of the reticuloendothelial system using quantum dots in combination with bioluminescent tumour monitoring", Eur J Nucl Med Mol Imaging 2007,

6 G. A. van Tilborg, W. J. Mulder, P. T. Chin, G. Storm, C. P. Reutelingsperger, K. Nicolay and G. J. Strijkers; "Annexin A5-Conjugated Quantum Dots with a Paramagnetic Lipidic Coating for the Multimodal Detection of Apoptotic Cells", Bioconjug Chem 2006, 17(4) 865-868.

7 K. Chen, Z.-B. Li, H. Wang, W. Cai and X. Chen; "Dual-modality optical and positron emission tomography imaging of vascular endothelial growth factor receptor on tumor vasculature using quantum dots", European Journal of Nuclear Medicine and Molecular Imaging 2008, 35(12) 2235-2244.

8 J. H. von der Thusen, T. J. C. van Berkel and E. A. L. Biessen; "Induction of Rapid Atherogenesis by Perivascular Carotid Collar Placement in Apolipoprotein E-Deficient and Low-Density Lipoprotein Receptor-Deficient Mice", Circulation 2001, 103(8) 1164-1170.

9 A. Dirksen, E. W. Meijer, W. Adriaens and T. M. Hackeng; "Strategy for the synthesis of multivalent peptide-based nonsymmetric dendrimers by native chemical ligation", Chem Commun (Camb) 2006, 15) 1667-9. 

Egbrink and M. A. van Zandvoort; "Two-Photon Microscopy of Vital Murine Elastic and Muscular Arteries. Combined Structural and Functional Imaging with Subcellular Resolution", J Vasc Res 2006, 44(2) 87-98.

R. Hardman; "A toxicologic review of quantum dots: toxicity depends on physicochemical and environmental factors", Environ Health Perspect 2006, 114(2) 165-72. Liu, F. Zhao, Y. L. Zhao and X. H. Fang; "Bio-distribution and metabolic paths of silica coated CdSeS quantum dots", Toxicology and Applied Pharmacology 2008, 230(3) 364-371.

H. C. Fischer, L. Liu, K. S. Pang and W. C. W. Chan; "Pharmacokinetics of Nanoscale Quantum Dots: In vivo Distribution, Sequestration, and Clearance in the Rat", Advanced Functional Materials 2006, 16(10) 1299-1305.

R. S. Yang, L. W. Chang, J. P. Wu, M. H. Tsai, H. J. Wang, Y. C. Kuo, T. K. Yeh, C. S. Yang and P. Lin; "Persistent tissue kinetics and redistribution of nanoparticles, quantum dot 705, in mice: ICP-MS quantitative assessment. " Environ Health Perspect 2007, 115(9) 1339-43. lial glycocalyx: composition, functions, and visualization", Pflugers Arch 2007,

L. Wang and S. M. Dudek; "Regulation of vascular permeability by sphingosine 1-phosphate", Microvascular Research 2009, In Press, Corrected Proof( S. A. Predescu, D. N. Predescu and A. B. Malik; "Molecular determinants of endothelial transcytosis and their role in endothelial permeability", Am J Physiol Lung Cell Mol Physiol 2007, 293(4) L823-842.

L. Wang and S. M. Dudek; "Regulation of Vascular Permeability by Sphingosine 1-phosphate", Microvasc Res 2008, In Press( A. Boueiz and P. M. Hassoun; "Regulation of endothelial barrier function by reactive oxygen and nitrogen species", Microvasc Res 2008, 2008, 103(5) 398-406. 
Chapter 5 


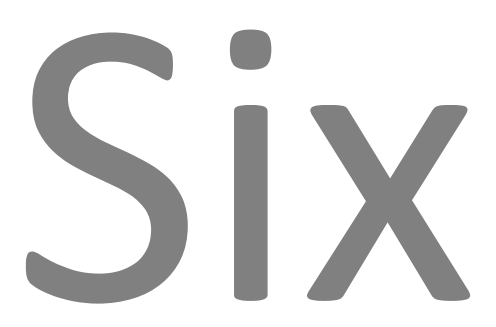

Molecular MRI of early thrombus formation using a bimodal $\alpha_{2}$-antiplasmin-based contrast-agent

JACC Cardiovascular Imaging, in press

RJJHM Miserus, VM Herías*, L Prinzen*, MBI Lobbes, RJ van Suylen, A Dirksen, TM Hackeng, JWM Heemskerk, JMA van Engelshoven, MJAP Daemen, MAMJ van Zandvoort, S Heeneman, ME Kooi

*Authors contributed equally 


\section{Abstract}

Objectives: We aimed to investigate whether early thrombus formation can be visualized with in vivo MRI using a novel bimodal $\alpha_{2}$-antiplasmin-based contrast agent (CA).

Background: Thrombus formation plays a central role in several vascular diseases and non-invasive detection of early thrombus formation is a major challenge in clinical practice. During the early phases of thrombus formation, activated factor XIII (FXIIIa) covalently cross-links $\alpha_{2}$-antiplasmin to fibrin, indicating the potential of $\alpha_{2}$-antiplasminbased CAs in detecting early thrombus formation.

Methods: A bimodal CA was synthesized by coupling gadolinium-DTPA and rhodamine to an $\alpha_{2}$-antiplasmin-based peptide. For the control CA, a glutamine residue essential for cross-linking was replaced by alanine. In vitro generated thrombi were exposed to both CAs and imaged by MRI and two-photon laser-scanning microscopy (TPLSM). Immunohistochemistry was performed on human pulmonary emboli sections to determine $\alpha_{2}$-antiplasmin and FXIII presence in different thrombus remodeling phases. In vivo feasibility of the $\mathrm{CA}$ in detecting early thrombus formation specifically, was investigated with MRI.

Results: In vitro generated thrombi exposed to the $\alpha_{2}$-antiplasmin-based CA showed hyperintense MR signal intensities at the thrombus edge. No hyperintense signal was observed using the $\alpha_{2}$-antiplasmin-based CA in the presence of FXIII inhibitor dansylcadaverine, nor when using the control CA. TPLSM demonstrated that the $\alpha_{2}$-antiplasminbased CA bound to fibrin. Immunohistochemistry demonstrated substantial $\alpha_{2}$ antiplasmin staining in fresh compared to lytic and organized thrombi. In vivo CA administration within seconds after inducing thrombus formation increased contrast-tonoise ratios (CNR: $2.28 \pm 0.39, \mathrm{~N}=6$ ) at the site of thrombus formation compared to the control CA (CNR: $-0.14 \pm 0.55, P=0.003, N=6$ ) and $\alpha_{2}$-antiplasmin-based CA administration 24-48 hours after thrombus formation (CNR: 0.11 $\pm 0.23, \mathrm{P}=0.006, \mathrm{~N}=6$ ).

Conclusions: A novel bimodal CA was developed, characterized, and validated. Our results showed that this bimodal CA enabled non-invasive in vivo MR visualization of early thrombus formation. 


\section{Introduction}

Thrombotic complications such as myocardial infarction, stroke, deep venous thrombosis, and pulmonary embolism, are major causes of morbidity and mortality ${ }^{1,2}$. The resistance of thrombi to fibrinolytic therapy increases with thrombus age ${ }^{3}$, and fibrinolytic agents can induce severe complications such as bleeding ${ }^{4,5}$. This indicates the necessity to accurately diagnose early thrombus formation as it may improve selection of patients that will benefit from fibrinolytic therapy. Currently, non-invasive detection of early thrombus formation is a major problem in clinical practice. Several specific contrast agents (CAs) have been developed to visualize thrombi using molecular imaging techniques ${ }^{6-8}$. Fibrin has frequently been used as a target for in vivo thrombus visualization using various magnetic resonance imaging (MRI) CAs, such as fibrin-targeted perfluorocarbon nanoparticles ${ }^{9}$, and fibrin-specific peptide-based CAs ${ }^{10-17}$. Recently, initial results of a human study using a fibrin-specific peptide-based CA (EP-2104R) were published, suggesting selective molecular MR imaging of thrombi ${ }^{18}$. Nonetheless, these CAs have low sensitivity for the estimation of thrombus age.

In this study, we searched for other coagulation factors that could be used as a target for thrombus imaging. We focused on activated factor XIII (FXIIIa) as it cross-links fibrin $\alpha$ - and $\gamma$-chains of neighboring fibrin molecules and covalently cross-links $\alpha_{2}$ antiplasmin $\left(\alpha_{2}-A P\right)$ to fibrin ${ }^{19}$. In this latter process, the glutamine $(G \ln 2)$ substrate in the $\mathrm{N}$-terminal domain of $\alpha_{2}$-AP is the primary substrate site for FXIIla ${ }^{20}$. Robinson et al. ${ }^{21}$ have shown that the catalytic ability of FXIIla in cross-linking $\alpha_{2}$-AP into formed thrombi declines with a short half-life. Therefore, a CA based on $\alpha_{2}$-AP might enable specific visualization of early thrombus formation.

Recently, a near-infrared (NIR) fluorescent probe (A15) and an MRI CA (A14), both based on $\alpha_{2}-A P$, enabled visualization of in vitro formed thrombi with NIR fluorescence and ex vivo MRI ${ }^{22}$. Subsequently, in vivo detection of FXIII activity was achieved in ferric chloride treated murine femoral vessels ${ }^{23}$ and in the superior sagittal sinus ${ }^{24}$ by intravital fluorescence microscopy using the A15 probe. However, translation of this probe to human studies in the near future is unlikely due to the limited penetration depth of NIR 


\section{Chapter 6}

fluorescence microscopy. Non-invasive thrombus visualization using imaging modalities such as MRI, may overcome this limitation.

In the present study, we first explored the feasibility of a bimodal $\alpha_{2}$-AP-based CA for visualization of in vitro formed thrombi with MRI and two-photon laser-scanning microscopy (TPLSM). Secondly, we analyzed the presence of FXIII and $\alpha_{2}$-AP in fresh, lytic and organized human pulmonary emboli using immunohistochemistry. Subsequently, we investigated whether early thrombus formation can be visualized in vivo with MRI using a bimodal $\alpha_{2}$-AP-based CA. Additionally, TPLSM was used to investigate whether the $\alpha_{2}$-AP based CA co-localizes with the fibrin polymers.

\section{Materials and methods}

\section{Synthesis of the CAs}

An $\alpha_{2}$-AP based peptide sequence (GNQEQVSPLTLL) that binds covalently to fibrin ${ }^{21,23}$ was synthesized using tertbutyloxycarbonyl (tBoc) solid-phase peptide synthesis (SPPS) ${ }^{25}$. The peptide was bimodally labeled with rhodamine and a DTPA-chelate (Bi- $\left.\alpha_{2} A P-C A\right)$ by cross-linking maleimide-DTPA ${ }^{26}$ and succinimidyl-rhodamine (Invitrogen, Molecular Probes, Breda, The Netherlands) to an additional C-terminal KW dipeptide, branched at the lysine $\varepsilon$-amino group with a cysteine. A control CA (Bi-con-CA) was obtained by replacing a glutamine residue essential for cross-linking by alanine ( $33 \rightarrow A 3)$ to prevent binding to fibrin (figure 1 ). Bi- $\alpha_{2} A P-C A$ and $B i-c o n-C A$ were characterized by matrixassisted laser desorption/ionization mass spectrometry (MALDI-MS). Gadolinium chloride $\left(\mathrm{GdCl}_{3}\right)$ was added in a 0.9:1 molar ratio after which no MALDI spectrum was observed due to poor ionization.

\section{Contrast agent validation}

For $r_{1}$ relaxivity measurements, the gadolinium-labeled peptides were solved (concentrations ranging from 25-150 $\mu \mathrm{M}$ ) in phosphate buffered saline solution (PBS). Solutions were imaged at room temperature with an 1.5 Tesla MR-scanner (MR Intera, Philips Healthcare, Best, The Netherlands) using a commercially available head coil and a 7.0 Tesla Bruker Biospec scanner (Bruker Biospin GmbH, Ettlingen, Germany) using a 154 

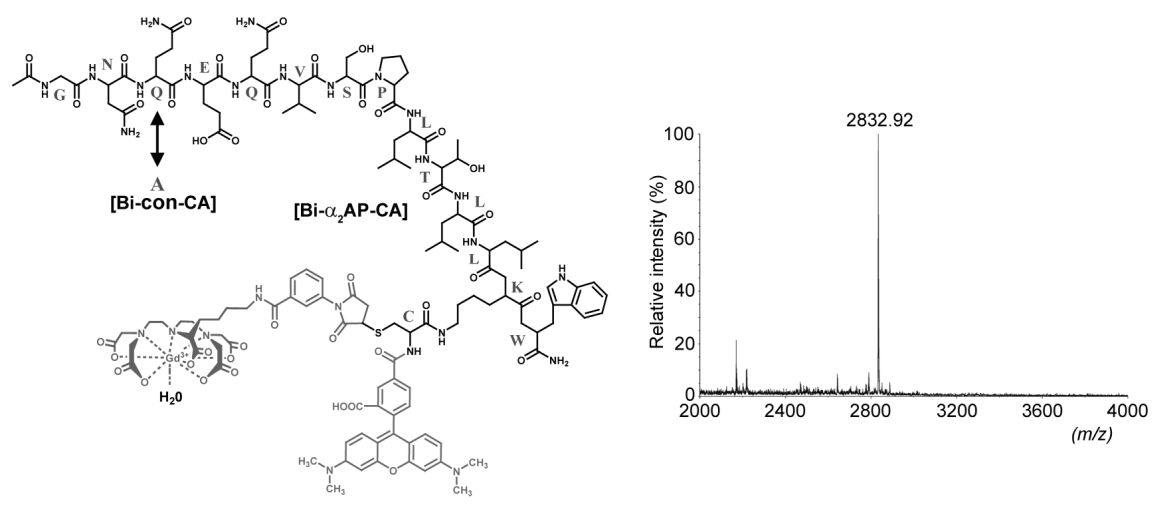

Figure 1: Schematic representation of the bimodal $\alpha_{2}-\mathrm{AP}$-based peptide labeled with rhodamine and DTPA (Bi$\alpha_{2}$ AP-CA) and the corresponding mass spectra. MALDI-MS shows a molecular mass of $2832.92 \mathrm{~g} / \mathrm{mol}$ for $\mathrm{Bi}-$ $\alpha_{2} A P-C A$. Substitution of one amino acid $(Q 3 \rightarrow A 3)$ results in a bimodal control $C A(B i-c o n-C A)$. Color figure on page 189.

$\mathrm{mm}$ diameter quadrature transmit-receive radio-frequency coil. $\mathrm{T}_{1}$ relaxation times were obtained using inversion recovery sequences with different inversion times (TI), ranging from 50-5000 ms. Additional parameters: repetition time (TR), $7500 \mathrm{~ms}$; echo time (TE), $14 \mathrm{~ms}$ (1.5T) or 8.4 (7.0T) ms; slice thickness, $3 \mathrm{~mm}$; number of signal averages (NSA), 1; in-plane resolution, $0.55 \times 0.55 \mathrm{~mm}^{2}$. Gadolinium content was determined using inductively coupled plasma mass spectrometry (ICP-MS).

The effect of $\mathrm{Bi}-\alpha_{2} \mathrm{AP}-\mathrm{CA}$ on thrombus formation was determined with a thrombin generation assay using platelet poor plasma with different concentrations $(0,10,20$, and 40 $\mu \mathrm{M})$ of $\mathrm{Bi}-\alpha_{2} \mathrm{AP}-\mathrm{CA}$.

\section{In vitro thrombus imaging}

Human blood was obtained by venous puncture from a healthy volunteer and collected in vacutainer tubes containing trisodium citrate (BD Vacutainer Systems, Preanalytical solutions, Plymouth, UK). Murine blood was obtained by right ventricle puncture. Human and murine thrombi were allowed to form during 90 minutes at $37^{\circ} \mathrm{C}$ in the presence of $30 \mu \mathrm{l}$ of $1.5 \mathrm{mg} / \mathrm{ml}$ Oregon Green 488-labeled fibrinogen (Invitrogen, Molecular Probes, Breda, The Netherlands). Thereafter, thrombi were incubated with $150 \mu \mathrm{M} \mathrm{Bi}$ $\alpha_{2} \mathrm{AP}-\mathrm{CA}$ or Bi-con-CA during 90 minutes at $37{ }^{\circ} \mathrm{C}$, followed by extensive washing with 


\section{Chapter 6}

PBS. To determine the effect of FXIIla on thrombus visualization, a human thrombus was formed and exposed to $\mathrm{Bi}-\alpha_{2} \mathrm{AP}-\mathrm{CA}$ in the presence of dansylcadaverine (final concentration $2 \mathrm{mM}$ ) (Fluka Chemie AG, Buchs, Switzerland), since dansylcadaverine is a competitive substrate for transglutaminases such as FXIII ${ }^{27}$.

Visualization with TPLSM was performed as previously described by Megens et al. ${ }^{28}$. Briefly, a BioRad 2100MP (Hemel Hampstead, UK) was used in TPLSM mode. Fluorescence was detected by 3 photomultipliers. Filter settings: $420-470 \mathrm{~nm}$ (blue), $510-$ $540 \mathrm{~nm}$ (green, fibrin network) and 570-590 nm (red, rhodamine detection of the CAs).

After TPLSM and approximately 4 hours after CA incubation, thrombi were embedded in $2 \%$ agarose gel and imaged at 1.5 Tesla using a commercially available $47 \mathrm{~mm}$ diameter surface coil (Philips Healthcare, Best, The Netherlands). Images were acquired using a $\mathrm{T}_{1}$-weighted Inversion Recovery Turbo Spin Echo (IR-TSE) sequence with the following scan-parameters: TR/TE/TI, 1580/13/546 ms; echo train length, 6; NSA, 16; slice thickness, $1.5 \mathrm{~mm}$; field of view (FOV), $40 \times 40 \mathrm{~mm}^{2}$; matrix size, 192x192; in-plane resolution, $0.21 \times 0.21 \mathrm{~mm}^{2}$.

\section{Thrombus age classification}

To assess whether FXIII and $\alpha_{2}$-AP can be seen as markers for early thrombus formation, tissue sections with pulmonary emboli were evaluated by immunohistochemistry (IHC). Twenty-two paraffin-embedded blocks (15 from 8 autopsy patients and 7 from 3 lung biopsy tissues) containing pulmonary emboli were used to analyze FXIII and $\alpha_{2}$-AP presence. All tissues were obtained from the Maastricht Pathology Tissue Collection (MPTC). Collection, storage, and use of tissue and patient data were performed in agreement with the "Code for Proper Secondary Use of Human Tissue in the Netherlands" (http://www.fmwv.nl).

Immunohistochemistry (IHC) was performed using conventional methods. Briefly, mouse monoclonal anti-human FXIII alpha subunit (1:300; clone AC-1A1, Lab Vision, Fremont, CA), rabbit-anti human $\alpha_{2}$-AP polyclonal (1:200; Biogenesis-MorphoSys AG, Martinsried/Munich, Germany), and mouse monoclonal anti-human fibrin II beta chain (1:100; clone T2G1, Accurate Chemical, Westbury, NY) were used as primary antibodies. For the negative controls, no primary antibody was used. Visualization was achieved 
with vectastain red (alkaline phosphatase substrate kit I, Vector Laboratories, Burlingame, CA).

Thrombi were classified into fresh ( $<1$ day; layered patterns of platelets, fibrin, erythrocytes, and intact granulocytes), lytic (1-5 days; areas of colliquation necrosis and karyorrhexis of granulocytes), organized ( $>5$ days; ingrowth of smooth muscle cells with or without deposition of connective tissue and capillary vessel ingrowth), or in thrombi containing more than one of these age classifications ${ }^{29}$. Percentages of thrombi positive for FXIII, $\alpha_{2}-A P$, and fibrin staining were determined for the various thrombus age classifications. Additionally, the degree of staining was scored for each thrombus age classification separately in no staining (score 0 ), slightly stained (score 1), positively stained (score 2), and strongly stained (score 3) (by VH, intraobserver variability $<10 \%$ ).

\section{Clearance and biodistribution}

All animal experiments were approved by the local ethical review committee. Mice were anesthetized by inhalation of air with $2 \%$ isoflurane inhalation gas. Half-life of $\mathrm{Bi}-$ $\alpha_{2}$ AP-CA ( $\left.n=3\right)$ and Bi-con-CA ( $\left.n=3\right)$ was determined by collecting blood samples before and at different time-points $(1,10,15,30,60$, and 90 minutes) after contrast administration (dose: $5.0 \mu \mathrm{mol} / \mathrm{kg}$ body weight). Using TPLSM, mean fluorescence of the red channel was determined in all blood samples. Exponential fitting was used for half-life determination (GraphPad Prism 4.0, La Jolla, USA). After blood collection, mice were sacrificed using an excess of pentobarbital. Biodistribution of both CAs was determined with TPLSM by studying CA uptake in excised liver, spleen, kidney, heart, lung, and intestine. Filter settings were: $\leq 500 \mathrm{~nm}$ (blue), 500-560nm (green) and 560-610nm (red, rhodamine detection of the CAs).

\section{In vivo thrombus imaging}

The right carotid artery was exposed by separating the sternocleidomastoid muscle from the trachea. Thrombus formation was induced by applying a strip of filter paper soaked in $10 \%$ ferric chloride $\left(\mathrm{FeCl}_{3}\right)$ on the carotid artery. After 5 minutes the filter paper was removed and the carotid artery was washed with PBS. Within seconds after $\mathrm{FeCl}_{3}$-induced thrombus formation, $5.0 \mu \mathrm{mol} / \mathrm{kg}$ body weight $\mathrm{Bi}-\alpha_{2} \mathrm{AP}-\mathrm{CA}(\mathrm{n}=6)$ or $\mathrm{Bi}$ - 


\section{Chapter 6}

con-CA ( $n=6)$ was administered intravenously. In 6 other mice, Bi- $\alpha_{2} A P-C A$ was administered 24-48 hours after $\mathrm{FeCl}_{3}$-induced thrombus formation.

MR imaging was performed at 7.0 Tesla using a $35 \mathrm{~mm}$ diameter quadrature transmitreceive radio-frequency coil (Bruker Biospin $\mathrm{GmbH}$, Ettlingen, Germany). After scout scans, sagittal carotid MR images were obtained approximately 90 minutes after CA administration using a rapid acquisition with relaxation enhancement (RARE) inversion recovery pulse sequence. Parameters were: TR/TE/TI, 7500/8.9/1654 ms; 21-25 slices; slice thickness, $0.25 \mathrm{~mm}$; interslice distance, 0.3-0.35 mm; NSA, 2; rare partitions, 4; FOV, $30 \times 30 \mathrm{~mm}^{2}$; matrix size, 312×312; in-plane resolution, $96 \times 96 \mu^{2}$. Additionally, after $\mathrm{Bi}-\alpha_{2} \mathrm{AP}-\mathrm{CA}$ administration within seconds after thrombus formation, repeated MR measurements were performed $(50,65,85,100,115$, and 130 minutes, $n=2)$.

For TPLSM, carotid arteries ( $n=4$ for each group) were excised and mounted into a home-built perfusion chamber (IDEE BV, Maastricht, The Netherlands), immersed in Hanks Balanced Salt Solution, maintaining $60-80 \mathrm{mmHg}$ transmural pressure ${ }^{28}$. Syto 13 (Invitrogen, Eugene, OR) was added (final concentration: $2.5 \mu \mathrm{M}$ ) to visualize nucleic acids. Filter settings: $420-470 \mathrm{~nm}$ (blue), 510-530 nm (green, nucleic acid staining) and $560-610 \mathrm{~nm}$ (red, rhodamine detection of the CAs).

MR contrast-to-noise ratios (CNRs) were calculated by dividing the difference between the mean MR SIs from a ROI positioned in the thrombus and that in neighboring muscle tissue by the noise measured in air. Noise values were corrected for magnitude effects by the Rayleigh factor. ROls were drawn in ParaVision version 4.0 (Bruker Biospin $\mathrm{GmbH}$, Ettlingen, Germany).

\section{Statistics}

Data are presented as mean \pm standard error. Statistical analyses were performed on the histology scores using the Mann-Whitney $\mathrm{U}$ test. For in vivo measurements the oneway ANOVA test was used. Due to Bonferroni correction for multiple group comparison, differences with a P-value less than 0.025 were considered significant. 


\section{Results}

\section{Synthesis of the CAs}

MALDI-MS showed a molecular mass for $\mathrm{Bi}-\alpha_{2} \mathrm{AP}-\mathrm{CA}$ of $2832.9 \mathrm{~g} / \mathrm{mol}$, in agreement with the theoretical average mass of $2893.1 \mathrm{~g} / \mathrm{mol}$ without $\mathrm{Gd}^{3+}$ bound (figure 1). The Bi-conCA with a Q3 to A mutation had a molecular mass of $2775.4 \mathrm{~g} / \mathrm{mol}$, corresponding to the theoretical average mass of $2776.1 \mathrm{~g} / \mathrm{mol}$ without $\mathrm{Gd}^{3+}$ attached (data not shown).

\section{Contrast agent validation}

The $r_{1}$ relaxation times of the Bi- $\alpha_{2} A P-C A$ at 1.5 and 7.0 Tesla were $5.6 \pm 0.4$ and $3.0 \pm 0.3$ $\mathrm{mM}^{-1} \mathrm{~s}^{-1}$, respectively. The $r_{1}$ relaxation times obtained for Bi-con-CA were $6.5 \pm 0.5 \mathrm{mM}$ ${ }^{1} \mathrm{~s}^{-1}(1.5 \mathrm{~T})$ and $3.3 \pm 0.2 \mathrm{mM}^{-1} \mathrm{~s}^{-1}(7.0 \mathrm{~T})$.

For all Bi- $\alpha_{2}$ AP-CA concentrations $(0,10,20$, and $40 \mu \mathrm{M})$ the endogenous thrombin potentials were comparable $(708,742,733$, and $717 \mathrm{nM} \cdot \mathrm{min}$, respectively). In addition, no effects of $\mathrm{Bi}-\alpha_{2} \mathrm{AP}-\mathrm{CA}$ on thrombin peak heights or lag times of thrombin generation were observed. These results indicate that $\mathrm{Bi}-\alpha_{2} \mathrm{AP}-\mathrm{CA}$ did not interfere with thrombin generation and subsequent thrombus formation.

\section{In vitro thrombus imaging}

TPLSM showed co-staining of the rhodamine-labeled Bi- $\alpha_{2} \mathrm{AP}-\mathrm{CA}$ (red) and the OG488labeled fibrin network (green) resulting in yellow areas of co-localization, indicating binding of $\mathrm{Bi}-\alpha_{2} \mathrm{AP}-\mathrm{CA}$ to fibrin in human and murine thrombi (figures $2 \mathrm{~B}$ and $\mathrm{H}$ ). In contrast, the control agent Bi-con-CA showed limited labeling and co-localization with the fibrin network (figures 2D and J). Hyperintense MR signals were found at the edge of thrombi incubated with $\mathrm{Bi}-\alpha_{2} \mathrm{AP}-\mathrm{CA}$ (figures $2 \mathrm{~A}$ and $\mathrm{G}$ ), which were absent using the $\mathrm{Bi}$ con-CA (figures $2 \mathrm{C}$ and I). FXIIla inhibition by dansylcadaverine decreased binding and co-localization of Bi- $\alpha_{2} A P-C A$ with fibrin, thereby lowering the MR signal intensity at the edge of a human thrombus exposed to $\mathrm{Bi}-\alpha_{2} \mathrm{AP}-\mathrm{CA}$ (figures $2 \mathrm{E}-\mathrm{F}$ ). This finding confirms that FXIII presence is needed for Bi- $\alpha_{2}$ AP-CA binding to fibrin. 


\section{Chapter 6}

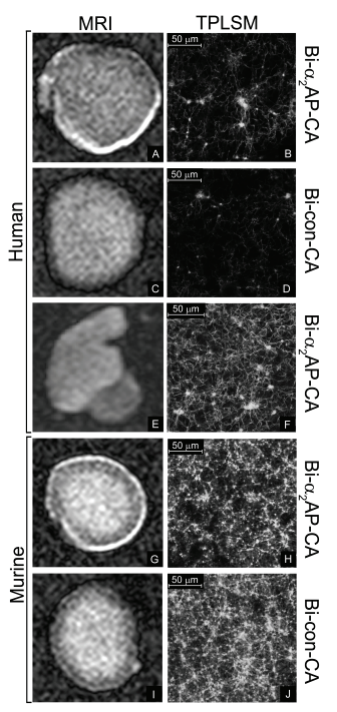

Figure 2: Two-photon laser-scanning microscopy (TPLSM) and magnetic resonance imaging (MRI) results for human (A-F) and murine (G-J) thrombi incubated with the bimodal $\alpha_{2}$-AP-based contrast agent ( $\left.\mathrm{Bi}-\alpha_{2} \mathrm{AP}-\mathrm{CA}\right)(\mathrm{A}, \mathrm{B}, \mathrm{E}-\mathrm{H})$ and the bimodal control CA (Bi-con-CA) (C, D, I, and J). For TPLSM, red color originated from the rhodamine-labeled CAs. Green color indicated the fibrin network due to use of fibrinogen-Oregon green 488. Yellow color illustrated co-localization of red and green. Hyperintense MR signals were found at the edge of thrombi incubated with Bi- $\alpha_{2} A P-C A(A, G)$. Lower MR signal intensities were found at the edge of a thrombus exposed to dansylcadaverine and $\mathrm{Bi}-$ $\alpha_{2} A P-C A(E)$. Color figure on page 189.

IHC staining for FXIII and $\alpha_{2}$-AP on a human thrombus formed during three hours showed that FXIII was present throughout the thrombus (figure $3 \mathrm{~A}$ ), while $\alpha_{2}$-AP was strongly expressed at the edge (figure 3B). This is in line with the increased MR signal intensities at the edge of thrombi exposed to $\mathrm{Bi}-\alpha_{2} \mathrm{AP}-\mathrm{CA}$.

\section{Thrombus age classification}

We evaluated 44 thrombi for FXIII, 38 for $\alpha_{2}-A P$, and 47 for fibrin and categorized them in fresh, lytic, or organized. High percentages of positive fibrin staining were found in all categories, whereas positive $\alpha_{2}-\mathrm{AP}$ and FXIII staining was mainly present in fresh thrombi followed by lytic and organized thrombi. Additionally, mean $\alpha_{2}$-AP score was significantly higher in fresh thrombi compared to lytic and organized thrombi, and mean FXIII score was significantly higher in fresh compared to organized thrombi while no significant difference was found between fresh and lytic thrombi. Mean fibrin score was relatively high in all thrombus age classifications (table 1). Additionally, FXIII was expressed in inflammatory cells (mainly macrophages), representing the cellular component of FXIII. A typical example of a thrombus containing fresh and organized components is shown in figure 4. 

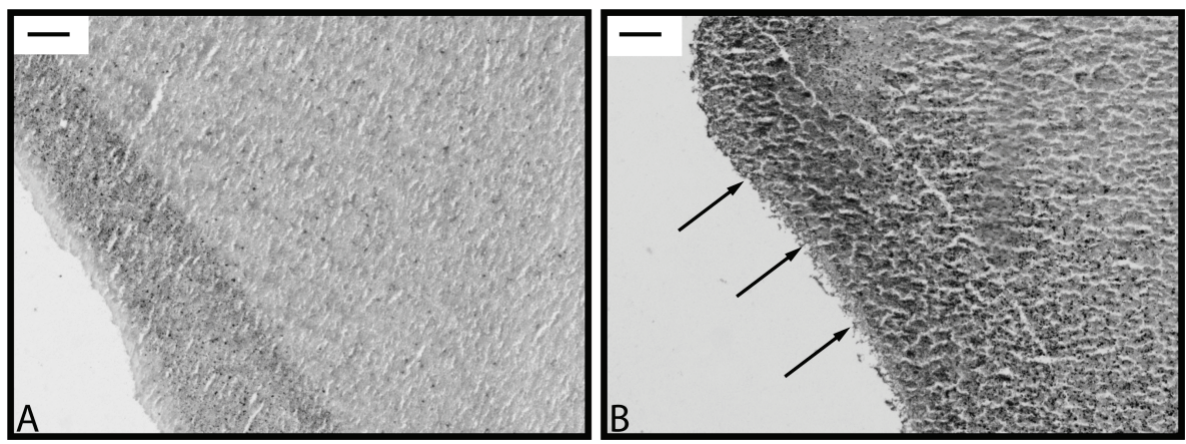

Figure 3: Immunohistochemical stainings of thrombi. A) factor XIII (FXIII) staining, B) $\alpha_{2}$-antiplasmin ( $\alpha_{2}$-AP) staining. Positive staining is depicted in red/pink color. FXIII staining (scanty spots) was present throughout the whole thrombus while $\alpha_{2}$-AP staining was mainly depicted at the edge of the thrombus (arrows). Bars: $50 \mu \mathrm{m}$. Color figure on page 190.

Table 1: Percentages and scores of $\alpha_{2}$-antiplasmin, factor XIII, and fibrin stainings in lung emboli tissue sections.

\begin{tabular}{|c|c|c|c|c|c|c|}
\hline & $\alpha_{2}$-antipl & $\operatorname{smin}$ & factor XI & & fibrin & \\
\hline Thrombus age & $\begin{array}{l}\text { Positive } \\
\text { staining }\end{array}$ & Mean score \pm s.e. & $\begin{array}{l}\text { Positive } \\
\text { staining }\end{array}$ & Mean score \pm s.e. & $\begin{array}{l}\text { Positive } \\
\text { staining }\end{array}$ & Mean score \pm s.e. \\
\hline Fresh (<1 day) & $95 \%$ & $1.4 \pm 0.1 \neg \neg$ & $92 \%$ & $1.5 \pm 0.1 \neg$ & $100 \%$ & $2.1 \pm 0.1$ \\
\hline Lytic (1-5 days) & $29 \%$ & $0.3 \pm 0.2$ & $75 \%$ & $0.8 \pm 0.3$ & $83 \%$ & $2.0 \pm 0.4$ \\
\hline $\begin{array}{l}\text { Organized (>5 } \\
\text { days) }\end{array}$ & $20 \%$ & $0.2 \pm 0.1$ & $31 \%$ & $0.3 \pm 0.1$ & $92 \%$ & $1.9 \pm 0.2$ \\
\hline
\end{tabular}

*Significantly different, s.e. indicates standard error

\section{Clearance and biodistribution}

Both CAs were rapidly cleared from the blood. Circulation half-lives of Bi- $\alpha_{2} A P-C A$ and Bi-con-CA were $14.1 \pm 8.4$ and $16.3 \pm 4.0$ minutes, respectively. TPLSM on excised organs showed that both CAs were mainly cleared through the kidneys, while limited CA was 


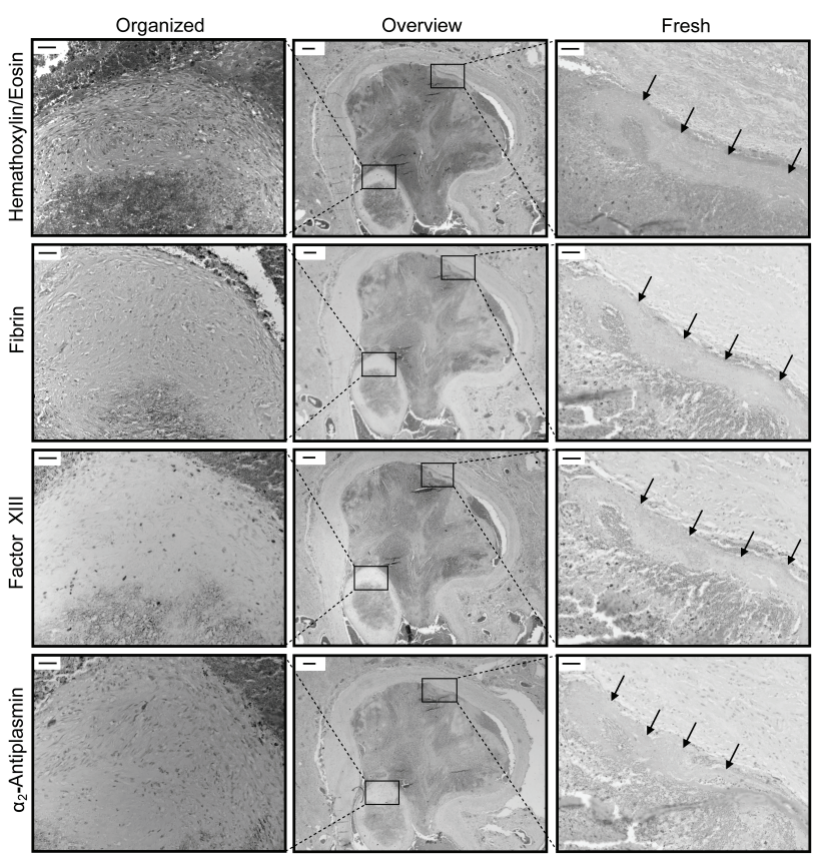

Figure 4: Lung emboli sections. Middle panels: overview of lung emboli, from top to bottom: Hemathoxyline/Eosin stained, immunohistochemically stained for fibrin, factor XIII, and $\alpha_{2}$-antiplasmin. Bars: $300 \mu \mathrm{m}$. Left panels: enlargements of boxed areas indicating an organized part of the lung emboli. In the enlarged FXIII stained area clear cellular FXIII staining is visable. Bars: $50 \mu \mathrm{m}$. Right panels: enlargements of boxed areas indicating a fresh segment (arrows). Bars: $50 \mu \mathrm{m}$. Color figure on page 190.

found in other organs (figure 5), indicating that there is no or limited accumulation of the CAs on organ level.

\section{In vivo thrombus imaging}

Clear hyperintense signals from early thrombus formation were observed with in vivo MRI (CNR: 2.29 \pm 0.39 ) when $\mathrm{Bi}-\alpha_{2} \mathrm{AP}-\mathrm{CA}$ was administered immediately after $\mathrm{FeCl}_{3}$ induced thrombus formation. No hyperintense MR signals were found using the control agent (Bi-con-CA) for fresh thrombus visualization (CNR: $-0.14 \pm 0.55, \mathrm{P}=0.003$ ) nor when using $\mathrm{Bi}-\alpha_{2} \mathrm{AP}-\mathrm{CA}$ for the visualization of $24-48$ hours old thrombi (CNR: $0.11 \pm 0.23$, $P=0.006$ ) (figures $6 A-D$ ). Additionally, CNR-ratios as a function of time after Bi- $\alpha_{2} A P-C A$ administration $(50,65,85,100,115$, and 130 minutes) were comparable $(2.65 \pm 0.11$, $2.43 \pm 0.06,2.51 \pm 0.15,2.43 \pm 0.37,2.24 \pm 0.21,2.01 \pm 0.44$, respectively).

TPLSM of excised carotid arteries confirmed $\mathrm{Bi}-\alpha_{2} \mathrm{AP}-\mathrm{CA}$ presence in the thrombi formed in the lumen of the $\mathrm{FeCl}_{3}$-treated artery when $\mathrm{Bi}-\alpha_{2} \mathrm{AP}-\mathrm{CA}$ was administered within seconds after thrombus formation (figure 6E). Limited fluorescence was found using Bi-con-CA (figure 6F) or when Bi- $\alpha_{2} \mathrm{AP}-\mathrm{CA}$ was administered 24-48 hours after 


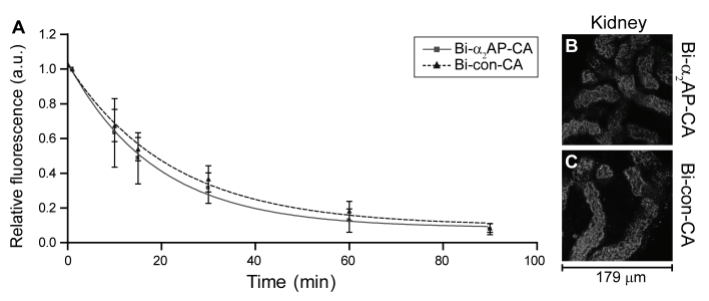

Figure 5: Clearance and kidney uptake of $\mathrm{Bi}-\alpha_{2} \mathrm{AP}-\mathrm{CA}$ and Bi-con-CA. Relative fluorescence measured in blood samples decreases with time after contrast administration (A). Half-life of the CAs is 14.1 \pm 8.4 and $16.3 \pm 4.0$ minutes for $\mathrm{Bi}$ $\alpha_{2} A P-C A$ and Bi-con-CA, respectively. Both CAs are mainly cleared through the kidney (B-C). Panels B-C: Red: Bimodal $C A$ present in kidney tubuli. Color figure on page 191.

$\mathrm{FeCl}_{3}$-induced thrombus formation (figure 6G). Occasionally, fluorescence was found in the medial layer (predominantly smooth muscle cells) of vessels that were treated with $\mathrm{FeCl}_{3}$ 24-48 hours prior to imaging. No fluorescence was detected in the non-treated contralateral carotid arteries. Histology confirmed presence of thrombi in all $\mathrm{FeCl}_{3}-$ treated carotid arteries.

\section{Discussion}

Since $\alpha_{2}$-AP covalently crosslinks to fibrin during the early phases of thrombus formation, $\alpha_{2}$-AP-based CAs might enable non-invasive detection of early thrombus formation. We demonstrated that the $\alpha_{2}$-AP-based CA enabled MR imaging and TPLSM of in vitro generated thrombi and that FXIII presence is required for binding of the $\alpha_{2}$-APbased CA to fibrin. Additionally, we showed that $\alpha_{2}$-AP and FXIII are predominantly present in fresh compared to lytic and organized human pulmonary emboli, indicating the potential of $\alpha_{2}$-AP-based CAs in detecting early thrombus formation. Subsequently, we demonstrated that the $\alpha_{2}$-AP-based CA enabled in vivo visualization of early thrombus formation with MRI. Two-photon laser-scanning microscopy confirmed specific binding of the $\alpha_{2}$-AP-based CA to fibrin.

Previously, $\alpha_{2}$-AP-based peptides labeled with either Alexa680 (A15) or gadolinium (A14) were used in vitro for visualization with NIR fluorescence microscopy and MRI. Both probes were tested for their ability to visualize murine ${ }^{22}$ and human thrombi ${ }^{23}$ by adding these probes during thrombus formation. Additionally, it was shown that $\alpha_{2}$-APbased peptides bind covalently to fibrin ${ }^{21,23}$, indicating that the $K_{d}$-value approaches 0 . 


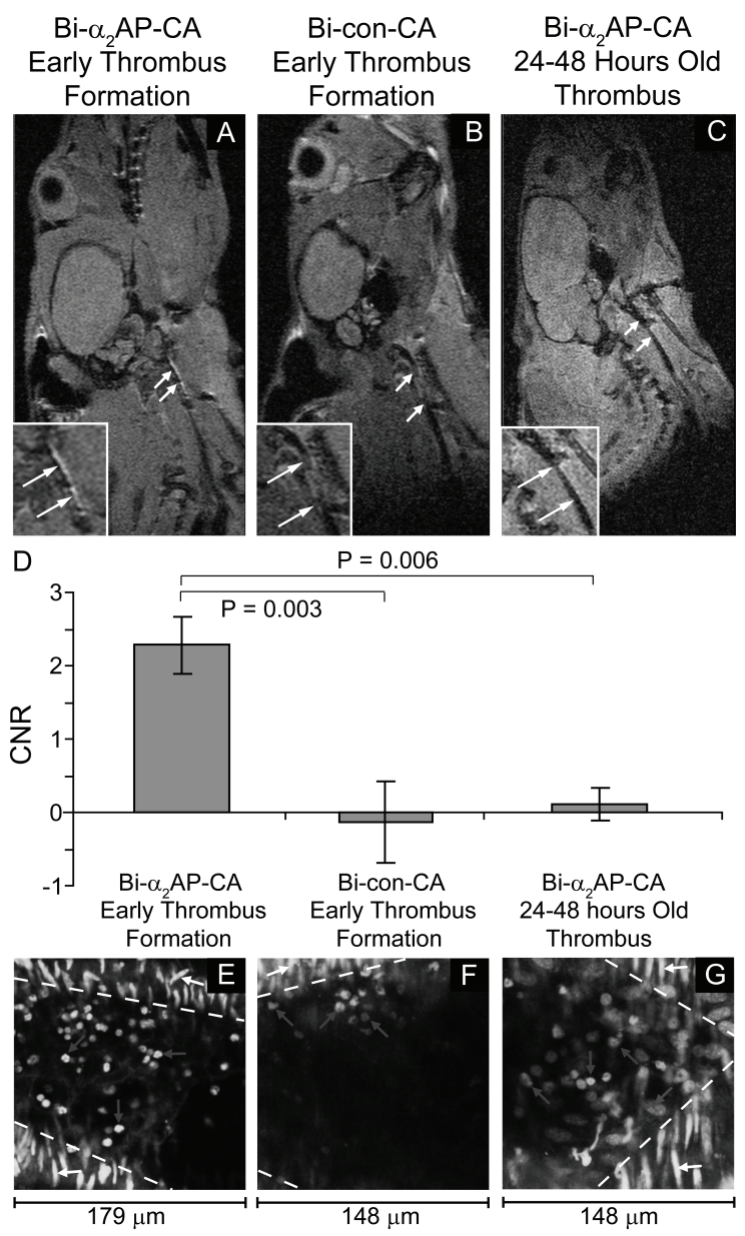

Figure 6: In vivo magnetic resonance images (MRI) (A-C) and ex vivo two-photon laser-scanning microscopy (TPLSM) results (E-G) of early thrombus formation and 24-48 hour old thrombi in murine carotid arteries after injection of the bimodal $\alpha_{2}$-antiplasmin-based CA (Bi- $\left.\alpha_{2} A P-C A\right)(A, C, E$, and $G$ ) and the bimodal control CA ( $B i-$ con- $C A$ ) ( $B$ and $F$ ). Contrast-to-noise ratios (CNR) are elevated in the early phases of thrombus formation after $\mathrm{Bi}-\alpha_{2} \mathrm{AP}-\mathrm{CA}$ administration (D). Panels E-G: Red: Bimodal CA. Green: Nuclear stain. Lumen is located between dotted lines. Blue arrows indicate leukocytes trapped in the thrombus. Elongated cells are smooth muscle cells (white arrows) and endothelial cells (red arrow). Color figure on page 191.

These findings were confirmed by the present study since contrast addition 90 minutes after the initiation of thrombus formation resulted in hyperintense MR signal at the edge of human as well as murine blood-derived thrombi.

In previous murine studies, intravital fluorescence microscopy enabled visualization of carotid ${ }^{23}$ and cerebral ${ }^{24}$ thrombi using the A15 probe. This probe proved to be specific 
for the early phases of thrombus formation ${ }^{23}$. Nevertheless, due to the limited penetration depth of NIR fluorescent microscopy it is unlikely that this probe can be used for human applications. Our $\alpha_{2}$-AP-based CA enabled in vivo visualization of early thrombus formation with non-invasive MRI. In our MRI study a higher CA dose was used compared to the NIR studies ${ }^{23,24}$. However, the present dose $(5 \mu \mathrm{mol} / \mathrm{kg})$ is still low for MRI. Biological amplification due to the abundance of fibrin and $r_{1}$ relaxivity increase of the CA upon binding may explain the observed signal enhancement achieved with this low dose. Other fibrin-targeted molecular imaging studies used comparable CA concentrations ${ }^{10-18}$. CAs like fibrin-targeted perfluorocarbon nanoparticles ${ }^{9}, \mathrm{EP}-1242{ }^{17}$, EP$1873^{16}$, and EP-2104R ${ }^{10-15,18}$ proved their ability to visualize thrombi in different animal models with MRI. Recently, initial results showed that EP-2104R allows in vivo MR imaging of thrombi in humans ${ }^{18}$. Additionally, EP-2104R demonstrated a time-dependent thrombus enhancement, showing a slow decrease in contrast-to-noise ratio (CNR) over a 8 weeks time period ${ }^{12}$. However, since increased CNRs were observed in all thrombi compared with pre-contrast imaging, it is unlikely that this CA could effectively discriminate between the early stages of thrombus formation and organized thrombi. In the present study, we demonstrated that the $\alpha_{2}$-AP-based CA significantly increased CNRs during early thrombus formation compared with 24-48 hours old thrombi. Therefore, our bimodal $\alpha_{2}$-AP-based CA is promising for the differentiation between the early phases of thrombus formation and organized thrombi.

Immunohistochemistry showed presence of FXIII in pulmonary thrombi, but cellular FXIII staining was also observed. The cellular form of FXIII consists of a homodimer of Asubunits ${ }^{19}$. We used a primary antibody addressed to the $\alpha$-chain of FXIII, explaining the cellular FXIII staining. The importance of FXIII beyond its function in coagulation is still not well known. An in vivo study, in which an ${ }^{111}$ In-labeled $\alpha_{2}$-AP-based CA was used to visualize FXIII activity in myocardial healing, suggested that FXIII plays a role in wound healing and tissue repair ${ }^{30}$. Therefore, the fluorescence observed in the medial layer of some carotid arteries might be explained by tissue repair 24-48 hours after $\mathrm{FeCl}_{3}$-treatment. Abdalla et al. ${ }^{31}$ suggested that intracellular FXIIla mediated monocyte adhesion during the atherosclerotic process. 


\section{Chapter 6}

Non-invasive assessment of early thrombus formation versus organized thrombi could be of major clinical relevance since specific detection of early thrombus formation may improve selection of patients eligible for fibrinolytic therapy, thereby reducing serious side-effects. Whether our CA is able to detect thrombi that are sensitive to fibrinolytic therapy remains to be determined. We have demonstrated that the $\alpha_{2}$-AP-based CA did not interfere with the thrombin generation, but it remains to be established whether this $\alpha_{2}$-AP-based CA inhibits thrombolysis. However, this CA only contains the part that is responsible for binding of $\alpha_{2}-A P$, which on its own is insufficient to inhibit plasmin activity. Finally, determination of the optimal dosage is still needed.

In conclusion, our bimodal $\alpha_{2}$-AP-based CA enabled specific, non-invasive visualization of the early phases of thrombus formation, while no hyperintense signal was observed in 24-48 hours old thrombi. Therefore, this bimodal $\alpha_{2}$-AP-based CA might be able to specifically select patients who are eligible for fibrinolytic therapy.

\section{Acknowledgements}

SH and MJAPD are members of the European Vascular Genomics Network (grant LSHMCT-2003-503254.). We thank Sander Langereis and Jeannette Smulders from Philips Research Eindhoven, The Netherlands for performing the ICP-MS measurements. This study was financially supported by the "Besluit Subsidies Investeringen Kennisinfrastructuur" (BSIK) program entitled Molecular Imaging of Ischemic Heart Disease (project number BSIK03033) and by the Dutch Heart Foundation, grant number 2002.B033. 


\section{References}

W. Rosamond, K. Flegal, G. Friday, K. Furie, A. Go, K. Greenlund, N. Haase, M. Ho, V. Howard, B. Kissela, S. Kittner, D. Lloyd-Jones, M. McDermott, J. Meigs, C. Moy, G. Nichol, C. J. O'Donnell, V. Roger, J. Rumsfeld, P. Sorlie, J. Steinberger, T. Thom, S. Wasserthiel-Smoller and Y. Hong; "Heart disease and stroke statistics--2007 update: a report from the American Heart Association Statistics Committee and Stroke Statistics Subcommittee", Circulation 2007, 115(5) e69-171.

R. H. White; "The epidemiology of venous thromboembolism", Circulation 2003, 107(23 Suppl 1) 14-8.

"Fibrinolytic Therapy Trialists' (FTT) Collaborative Group. Indications for fibrinolytic therapy in suspected acute myocardial infarction: collaborative overview of early mortality and major morbidity results from all randomised trials of more than 1000 patients. " Lancet 1994, 343(8893) 311-22.

R. A. Lange and L. D. Hillis; "Reperfusion therapy in acute myocardial infarction", N Engl J Med 2002, 346(13) 954-5.

L. B. Goldstein; "Acute ischemic stroke treatment in 2007", Circulation 2007, 116(13) 1504-14.

R. J. J. H. M. Miserus, S. Heeneman, J. M. A. v. Engelshoven, M. E. Kooi and M. J. A. P. Daemen; "Development and validation of novel imaging technologies to assist translational studies in atherosclerosis", Drug Disc. Today: Techn. 2006, 3(2) 195-204.

S. A. Wickline, A. M. Neubauer, P. Winter, S. Caruthers and G. Lanza; "Applications of nanotechnology to atherosclerosis, thrombosis, and vascular biology", Arterioscler Thromb Vasc Biol 2006, 26(3) 435-41.

F. A. Jaffer and R. Weissleder; "Molecular imaging in the clinical arena", Jama 2005, 293(7) 855-62.

S. Flacke, S. Fischer, M. J. Scott, R. J. Fuhrhop, J. S. Allen, M. McLean, P. Winter, G. A. Sicard, P. J. Gaffney, S. A. Wickline and G. M. Lanza; "Novel MRI contrast agent for molecular imaging of fibrin: implications for detecting vulnerable plaques", Circulation 2001, 104(11) 1280-5.

R. M. Botnar, A. Buecker, A. J. Wiethoff, E. C. Parsons, Jr., M. Katoh, G. Katsimaglis, R. M. Weisskoff, R. B. Lauffer, P. B. Graham, R. W. Gunther, W. J. Manning and E. Spuentrup; "In vivo magnetic resonance imaging of coronary thrombosis using a fibrin-binding molecular magnetic resonance contrast agent", Circulation 2004, 110(11) 1463-6. 


\section{Chapter 6}

11

E. Spuentrup, A. Buecker, M. Katoh, A. J. Wiethoff, E. C. Parsons, Jr., R. M. Botnar, R. M. Weisskoff, P. B. Graham, W. J. Manning and R. W. Gunther; "Molecular magnetic resonance imaging of coronary thrombosis and pulmonary emboli with a novel fibrin-targeted contrast agent", Circulation 2005, 111(11) 1377-82.

2 M. Sirol, V. Fuster, J. J. Badimon, J. T. Fallon, P. R. Moreno, J. F. Toussaint and Z. A. Fayad; "Chronic thrombus detection with in vivo magnetic resonance imaging and a fibrin-targeted contrast agent", Circulation 2005, 112(11) 1594-600.

E. Spuentrup, B. Fausten, S. Kinzel, A. J. Wiethoff, R. M. Botnar, P. B. Graham, S. Haller, M. Katoh, E. C. Parsons, Jr., W. J. Manning, T. Busch, R. W. Gunther and A. Buecker; "Molecular magnetic resonance imaging of atrial clots in a swine model", Circulation 2005, 112(3) 396-9.

E. Spuentrup, M. Katoh, A. Buecker, B. Fausten, A. J. Wiethoff, J. E. Wildberger, P. Haage, E. C. Parsons, Jr., R. M. Botnar, P. B. Graham, M. Vettelschoss and R. W. Gunther; "Molecular MR Imaging of Human Thrombi in a Swine Model of Pulmonary Embolism Using a Fibrin-Specific Contrast Agent", Invest Radiol 2007, 42(8) 586-595.

C. P. Stracke, M. Katoh, A. J. Wiethoff, E. C. Parsons, P. Spangenberg and E. Spuntrup; "Molecular MRI of cerebral venous sinus thrombosis using a new fibrin-specific MR contrast agent", Stroke 2007, 38(5) 1476-81.

R. M. Botnar, A. S. Perez, S. Witte, A. J. Wiethoff, J. Laredo, J. Hamilton, W. Quist, E. C. Parsons, Jr., A. Vaidya, A. Kolodziej, J. A. Barrett, P. B. Graham, R. M. Weisskoff, W. J. Manning and M. T. Johnstone; "In vivo molecular imaging of acute and subacute thrombosis using a fibrin-binding magnetic resonance imaging contrast agent", Circulation 2004, 109(16) 2023-9. M. Sirol, J. G. Aguinaldo, P. B. Graham, R. Weisskoff, R. Lauffer, G. Mizsei, I. Chereshnev, J. T. Fallon, E. Reis, V. Fuster, J. F. Toussaint and Z. A. Fayad; "Fibrin-targeted contrast agent for improvement of in vivo acute thrombus detection with magnetic resonance imaging", Atherosclerosis 2005, 182(1) 79-85.

E. Spuentrup, R. M. Botnar, A. J. Wiethoff, T. Ibrahim, S. Kelle, M. Katoh, M. Ozgun, E. Nagel, J. Vymazal, P. B. Graham, R. W. Gunther and D. Maintz; "MR imaging of thrombi using EP2104R, a fibrin-specific contrast agent: initial results in patients", Eur Radiol 2008,

L. Muszbek, V. C. Yee and Z. Hevessy; "Blood coagulation factor XIII: structure and function", Thromb Res 1999, 94(5) 271-305.

K. N. Lee, C. S. Lee, W. C. Tae, K. W. Jackson, V. J. Christiansen and P. A. McKee; "Crosslinking of alpha 2-antiplasmin to fibrin", Ann N Y Acad Sci 2001, 936(335-9. 
B. R. Robinson, A. K. Houng and G. L. Reed; "Catalytic life of activated factor XIII in thrombi. Implications for fibrinolytic resistance and thrombus aging", Circulation 2000, 102(10) 11517.

C. H. Tung, N. H. Ho, Q. Zeng, Y. Tang, F. A. Jaffer, G. L. Reed and R. Weissleder; "Novel factor XIII probes for blood coagulation imaging", Chembiochem 2003, 4(9) 897-9. Weissleder; "Molecular imaging of factor XIIla activity in thrombosis using a novel, nearinfrared fluorescent contrast agent that covalently links to thrombi", Circulation 2004, 110(2) $170-6$.

D. E. Kim, D. Schellingerhout, F. A. Jaffer, R. Weissleder and C. H. Tung; "Near-infrared fluorescent imaging of cerebral thrombi and blood-brain barrier disruption in a mouse model of cerebral venous sinus thrombosis", J Cereb Blood Flow Metab 2005, 25(2) 226-33.

M. Schnolzer, P. Alewood, A. Jones, D. Alewood and S. B. Kent; "In situ neutralization in Bocchemistry solid phase peptide synthesis. Rapid, high yield assembly of difficult sequences", Int J Pept Protein Res 1992, 40(3-4) 180-93.

A. Dirksen, S. Langereis, B. F. de Waal, M. H. van Genderen, E. W. Meijer, Q. G. de Lussanet and T. M. Hackeng; "Design and synthesis of a bimodal target-specific contrast agent for angiogenesis", Org Lett 2004, 6(26) 4857-60.

S. Kulkarni and S. P. Jackson; "Platelet factor XIII and calpain negatively regulate integrin alphallbbeta3 adhesive function and thrombus growth", J Biol Chem 2004, 279(29) 30697706.

R. T. Megens, S. Reitsma, P. H. Schiffers, R. H. Hilgers, J. G. De Mey, D. W. Slaaf, M. G. oude Egbrink and M. A. van Zandvoort; "Two-photon microscopy of vital murine elastic and muscular arteries. Combined structural and functional imaging with subcellular resolution", J Vasc Res 2007, 44(2) 87-98.

S. Z. Rittersma, A. C. van der Wal, K. T. Koch, J. J. Piek, J. P. Henriques, K. J. Mulder, J. P. Ploegmakers, M. Meesterman and R. J. de Winter; "Plaque instability frequently occurs days or weeks before occlusive coronary thrombosis: a pathological thrombectomy study in primary percutaneous coronary intervention", Circulation 2005, 111(9) 1160-5.

M. Nahrendorf, K. Hu, S. Frantz, F. A. Jaffer, C. H. Tung, K. H. Hiller, S. Voll, P. Nordbeck, D. Sosnovik, S. Gattenlohner, M. Novikov, G. Dickneite, G. L. Reed, P. Jakob, A. Rosenzweig, W. R. Bauer, R. Weissleder and G. Ertl; "Factor XIII deficiency causes cardiac rupture, impairs wound healing, and aggravates cardiac remodeling in mice with myocardial infarction", Circulation 2006, 113(9) 1196-202. 


\section{Chapter 6}

31

S. AbdAlla, H. Lother, A. Langer, Y. el Faramawy and U. Quitterer; "Factor XIIIA transglutaminase crosslinks AT1 receptor dimers of monocytes at the onset of atherosclerosis", Cell 2004, 119(3) 343-54. 


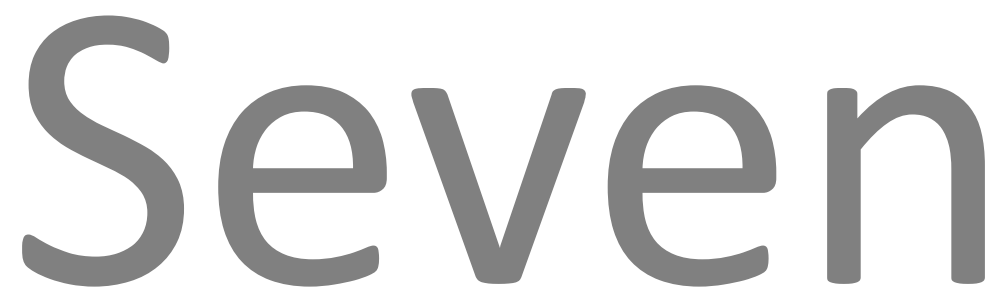

General discussion 


\section{Chapter 7}

In this thesis, the feasibility of bimodal imaging of vulnerable plaques in murine carotid arteries was investigated. Three different molecular target/ligand combinations based on two different nanoparticles were explored (Anx-Qdot, CNA-Qdot, and Bi- $\alpha_{2} A P-C A$ ) for their use in imaging with fluorescence microscopy and magnetic resonance imaging (MRI). The fluorescence microscopy imaging technique used in this thesis was twophoton laser scanning microscopy (TPLSM); a technique with several advantages, such as a larger penetration depth, less bleaching, and less tissue damage due to reduced laser-induced heat development. Dying cells, collagen, and fresh thrombi were used as targets of vulnerable plaques. The bimodal nanoparticles were applied to mouse models of atherosclerosis and thrombosis and imaged both in vivo with MRI and ex vivo with TPLSM. TPLSM allowed accurate cellular localization of the nanoparticles within the plaque or thrombus. MRI allowed minor visualization of CNA-Qdot and good visualization of $\mathrm{Bi}-\alpha_{2} \mathrm{AP}-\mathrm{CA}$ within the carotid arteries at tissue level. Anx-Qdot could not be visualized with MRI.

Qdots are nanoparticles with excellent fluorescent properties. They are very bright, hardly bleach, and have favorable excitation and emission spectra. Disadvantages are their potential toxicity, low stock concentration as provided by the manufacturer, and their high costs. In chapters 4 and 5, Qdots were utilized as bimodal nanoparticles by adding Gd-DTPA wedges, first in an in vitro/ex vivo proof-of-principle study and next in an in vivo atherosclerosis study. Two different ligands were explored. CNA targets collagen present in atherosclerotic plaques as was already shown previously in two TPLSM studies ${ }^{1,2}$, while Anx targets phosphatidylserine (PS) exposed by dying cells and activated platelets ${ }^{3,4}$. TPLSM results showed Anx-Qdots labeling plaque endothelium as well as distinct regions and foam cells within lipid pools. CNA-Qdots were visualized mainly on fibrous caps just underneath the endothelium in plaque areas and were also observed inside plaque on one occasion. These target-specific results strongly indicate that Qdots are able to enter plaques only at sites with increased permeability, presumably related to plaque vulnerability. Visualization with MRI should be further optimized. This is discussed below.

In chapter 6 TPLSM results showed that peptide-based Bi- $\alpha_{2}$ AP-CA specifically targeted activated factor XIII and co-localizes with fibrin. This was shown both in in vitro whole 
blood clots and in carotid arteries containing thrombi after $\mathrm{FeCl}_{3}$ treatment and intravenous injection with the bimodal contrast agent. Furthermore, Bi- $\alpha_{2} \mathrm{AP}-\mathrm{CA}$ was proven to be specific for fresh thrombi; thrombi of $24-48 \mathrm{~h}$ old were not targeted by Bi- $\alpha_{2} \mathrm{AP}-\mathrm{CA}$. Information about thrombus age is important in fibrinolytic treatment after infarctions. MRI results showed a contrast-to-noise ratio $(C N R)$ of $\approx 2.5$ for $B i-\alpha_{2} A P-C A$ in fresh thrombi, which is sufficient for visualization.

For MRI, visualization of nanoparticles is more difficult than for TPLSM as shown in chapters 4 and 5 , because sensitivity of MRI is relatively low. Many factors play a role in the potential of functionalized nanoparticles to visualize vulnerable plaques, and should be optimized for MRI visualization. First, the bioavailability of the target (both the number of targets present and the location of the target) is important. Fibrin and activated factor XIII are targets readily available in the vascular lumen in high amounts in case of active thrombus formation, in contrast to collagen and PS that are localized underneath the endothelium and presumably present to a lower extent than fibrin.

Second, the affinity of the ligand for the target plays an important role. Binding of Anx and CNA on one hand and $\alpha_{2}$-AP on the other to their respective targets are based on different biochemical processes. Anx and CNA (chapter 5) are ligands that bind their targets (PS and collagen, respectively) through dis/association equilibria with high affinities. Affinity of a ligand for its target is described by its dissociation constant $\left(K_{d}\right)$, which is defined as the concentration of ligand at which half of the number of target sites is occupied with the ligand; the lower this concentration the higher its affinity. For both CNA and Anx (chapter 5) these affinities are high, in the nanomolar range ${ }^{3-5}$. In the case of thrombus formation (chapter 6), $20-30 \%$ of target sites on fibrin are occupied with $\alpha_{2}-\mathrm{AP}^{6}$ through covalent coupling, catalyzed by activated factor XIII ${ }^{7}$. The overall reaction rate in enzyme kinetics is described by its Michaelis constant, $\mathrm{K}_{m}$, defined as the concentration of substrate at which the reaction rate is half maximum; or, the lower this concentration the faster this reaction may occur. For $\alpha_{2}$-AP coupling to fibrin catalyzed by activated factor XIII, $\mathrm{K}_{\mathrm{m}}$ is $\approx 5 \mu \mathrm{M}^{8}$, which is reasonably low for enzymes in general $^{9}$. All three target/ligand combinations are therefore biochemically suitable for utilization in molecular imaging. 


\section{Chapter 7}

Third, the availability (dose and circulation half-life) of the nanoparticle, and in case of MRI the Gd-content of this nanoparticle, is crucial. The total injected dose of Gd in the $\alpha_{2}$-AP-study (chapter 6 ) was approximately 4 times higher than in the bimodal Qdot study (chapter 5), despite the use of Gd-DTPA wedges that amplified the number of GdDTPA moieties by a factor eight. Furthermore, in both studies the circulation half-lives were short, reducing the chance of the nanoparticle to reach its target before being cleared from the circulation; $\approx 5 \mathrm{~min}$ for CNA- and Anx-Qdots and $\approx 15 \mathrm{~min}$ for $\mathrm{Bi}-\alpha_{2} \mathrm{AP}$ CA. Altogether, availability of Gd-DTPA in the thrombus study was approximately 12 times higher than in the atherosclerosis study.

Fourth, the size of the nanoparticle contributes to the amount of labeling. Bi- $\alpha 2 A P-C A$ $(\approx 3 \mathrm{kD}$, or $<<1 \mathrm{~nm})$ is much smaller than the bimodal Qdots $(\approx 10 \mathrm{~nm})$. The size of the nanoparticle mainly plays a role when the target is subendothelial, such as in the atherosclerosis study (chapter 5), because then the nanoparticle has to pass through the endothelium or intimal layer. Endothelial cells are, in healthy condition, neatly arranged. This means that nanoparticles up to $2 \mathrm{~nm}{ }^{10}$ are able to passively diffuse through the endothelium into the subendothelial space. Most nanoparticles are larger (see chapter 2), such as the bimodal Qdots used in chapter 5, and are thus unable to label subendothelial targets when the vascular wall is intact. However, endothelial permeability increases when tissue underlying the endothelium becomes inflamed, such as in atherosclerosis. As the plaque progresses into an advanced lesion and cells within the plaque release more cytokines and enzymes such as matrix degrading enzymes (matrix metalloproteinases), pathologic disruption of endothelium and fibrous cap occurs, leading to even larger gaps. The ultimate event of atherosclerotic plaque development may be plaque rupture, a process in which endothelium and underlying fibrous cap are completely destructed. Targeting subendothelial objects by sizeselection is, in addition to molecular targeting, a tool in development of an appropriate nanoparticle for the visualization of vulnerable atherosclerotic plaques, especially in subendothelial targets, as was shown in chapter 5 . In other words, the size of the nanoparticle may be adjusted to allow specific labeling of sites with a certain amount of endothelial or fibrous cap damage. Future experiments may utilize Qdots of various 
sizes $\left(\approx 5-20 \mathrm{~nm}^{11}\right)$, and hence, different emission spectra, to show size-specific labeling of atherosclerotic plaques.

These four factors combined, it may be concluded that due to the higher bioavailability of factor XIII/fibrin, and due to the higher availability of Gd-DTPA, the in vivo thrombus study described in chapter 6 lead to better results with MRI than in the atherosclerosis model described in chapter 5 . An additional cause of the disappointing MRI results of the Qdot study is that CNA- and Anx-Qdots were found only locally within plaques. This hot-spot labeling of CNA- and Anx-Qdots hinders MRI visualization, as resolution is $\approx$ $100 \mu \mathrm{m}$, about the size of these labeled spots.

In vivo atherosclerosis imaging studies with MRI have been performed previously, either non-targeted ${ }^{12-14}$, or targeting angiogenesis ${ }^{15,16}$, macrophages ${ }^{17-19}$, dying cells ${ }^{20}$, matrix metalloproteinases ${ }^{21}$, activated platelets ${ }^{22}$, or adhesion molecules ${ }^{23}$. Most of these studies used Gd-DTPA or Gd-DOTA as MRI contrast agent. In these studies a higher dose of Gd was used compared to the dose used in chapters 5 or 6 , and/or had slower clearance rates. Often lipidic structures such as micelles or liposomes were used, that can contain up to tens of thousands of Gd-moieties. A common disadvantage of lipidic structures is the relatively high unspecific binding ${ }^{16,17,19}$ that is presumably related to merging of lipids with cellular membranes. Unspecific binding is proven by the fact that untargeted liposomes also label atherosclerotic plaques ${ }^{14}$. This hinders specific molecular targeting and accurate discrimination between stable and vulnerable plaques. Other studies used iron-containing particles, inducing negative contrast (reduction of signal intensity). Bimodal Qdots used in chapter 5 have a high $r_{1}$ relaxivity of 3000-4500 $\mathrm{mM}^{-1} \mathrm{~s}^{-}{ }^{1}$ per particle; higher than other Anx-functionalized nanoparticles with respect to size (see chapter 4). Furthermore, aspecific binding to atherosclerotic plaques is negligible, as can be seen on TPLSM images in chapter 5. However, due to the low injected dose MRI visualization was not optimal. Of course, with increasing dose aspecific binding may also increase. Future experiments will show whether custommade, high-dose bimodal Qdots are able to improve visualization of vulnerable plaques with both TPLSM and MRI.

It is difficult to say which target/ligand combination would be optimal for the imaging of vulnerable plaques. On one hand, target should be readily available to allow sufficient 


\section{Chapter 7}

labeling. On the other hand, the target should be specific for vulnerable plaques; in the ideal case stable and vulnerable plaques are perfectly distinguishable. So far, a single target that predicts plaque vulnerability has not yet been discovered. The presence of thrombus is obvious for the presence of vulnerable plaque, though not necessarily at the same location. Whether Anx or CNA are ligands specific for vulnerable plaques still remains to be elucidated; there is one study concerning Anx in patients that points towards its potency to discriminate between plaque vulnerability and stability ${ }^{24}$. As discussed above and in chapter 5 , combining specific molecular targets with size-selective targeting may improve the specific visualization of targets inside vulnerable plaques.

As discussed in chapter 2 , not many in vivo studies have been performed using optical techniques. Non-optical and non-invasive techniques such as MRI, PET, and SPECT are more common in the clinical setting and more likely to implement molecular imaging. On the other hand, endoscope-based techniques such as OCT or TPLSM may provide complementary information due to the higher resolution. In the future, optical techniques may very well enter the clinical setting as well. As non-invasive imaging techniques are concerned, MRI is a promising technique to allow clinical molecular imaging, due to the relatively good resolution (compared to PET/SPECT/ultrasound), the lack of radiation (as opposed to $\mathrm{PET} / \mathrm{SPECT} / \mathrm{CT}$ ), and anatomical information (as opposed to PET/SPECT). A disadvantage is the low sensitivity to contrast agents. Nanoparticles should be optimized for visualization with MRI, as discussed above. An alternative to MRI may be PET imaging in the case of low-signal enhancement. Gd-DTPA used in this thesis may easily be replaced by $G a^{68}$-DTPA, a PET contrast agent. Future experiments may thus include combined PET/TPLSM experiments.

Concluding, this thesis provides novel bimodal nanoparticles for the specific visualization of vulnerable atherosclerotic plaques with TPLSM and MRI. Future research will show if the described bimodal Qdots will allow improved MRI visualization and if molecular imaging of vulnerable plaques is clinically applicable. 


\section{References}

R. T. Megens, M. G. Oude Egbrink, J. P. Cleutjens, M. J. Kuijpers, P. H. Schiffers, M. Merkx, D. W. Slaaf and M. A. van Zandvoort; "Imaging Collagen in Intact Viable Healthy and Atherosclerotic Arteries Using Fluorescently Labeled CNA35 and Two-Photon Laser Scanning Microscopy", Mol Imaging 2007, 6(4) 247-60.

R. T. A. Megens, M. G. A. o. Egbrink, M. Merkx, D. W. Slaaf and M. A. M. J. van Zandvoort; "Two-photon microscopy on vital carotid arteries: imaging the relationship between collagen and inflammatory cells in atherosclerotic plaques", Journal of Biomedical Optics 2008, 13(4) 044022-10.

C. P. Reutelingsperger and W. L. van Heerde; "Annexin V, the regulator of phosphatidylserine-catalyzed inflammation and coagulation during apoptosis", Cell Mol Life Sci 1997, 53(6) 527-32.

J. F. Tait and D. Gibson; "Phospholipid binding of annexin V: effects of calcium and membrane phosphatidylserine content", Arch Biochem Biophys 1992, 298(1) 187-91.

K. N. Krahn, C. V. Bouten, S. van Tuijl, M. A. van Zandvoort and M. Merkx; "Fluorescently labeled collagen binding proteins allow specific visualization of collagen in tissues and live cell culture", Anal Biochem 2006, 350(2) 177-85.

M. Mosesson, K. Siebenlist, I. Hernandez, K. Lee, V. Christiansen and P. Mckee; "Evidence that 2-antiplasmin becomes covalently ligated to plasma fibrinogen in the circulation: a new role for plasma factor XIII in fibrinolysis regulation", Journal of Thrombosis and Haemostasis 2008, 6(9) $1565-1570$.

L. Muszbek, V. C. Yee and Z. Hevessy; "Blood Coagulation Factor XIII: Structure and Function", Thrombosis Research 1999, 94(5) 271-305.

K. Lee, C. Lee, W. Tae, K. Jackson, V. Christiansen and P. Mckee; "Crosslinking of 2Antiplasmin to Fibrin", Ann N Y Acad Sci 2001, 936(FIBRINOGEN: XVIth INTERNATIONAL FIBRINOGEN WORKSHOP) 335-339.

J. Berg, J. Tymoczko, L. Stryer and N. Clarke, "Biochemistry", 5th edition, 2002, W. H. Freeman and Company. scytosis and their role in endothelial permeability", Am J Physiol Lung Cell Mol Physiol 2007, 293(4) L823-842. 


\section{Chapter 7}

11

B. N. Giepmans, T. J. Deerinck, B. L. Smarr, Y. Z. Jones and M. H. Ellisman; "Correlated light and electron microscopic imaging of multiple endogenous proteins using Quantum dots", Nat Methods 2005, 2(10) 743-9.

K. C. Briley-Saebo, V. Mani, F. Hyafil, J. C. Cornily and Z. A. Fayad; "Fractionated Feridex and positive contrast: in vivo MR imaging of atherosclerosis", Magn Reson Med 2008, 59(4) 72130.

S. P. Howarth, T. Y. Tang, M. J. Graves, U. K.-I. JM, Z. Y. Li, S. R. Walsh, M. E. Gaunt and J. H. Gillard; "Non-invasive MR imaging of inflammation in a patient with both asymptomatic carotid atheroma and an abdominal aortic aneurysm: a case report", Ann Surg Innov Res 2007, $1(4$.

W. J. Mulder, K. Douma, G. A. Koning, M. A. van Zandvoort, E. Lutgens, M. J. Daemen, K. Nicolay and G. J. Strijkers; "Liposome-enhanced MRI of neointimal lesions in the ApoE-KO mouse", Magn Reson Med 2006, 55(5) 1170-4.

C. Burtea, S. Laurent, O. Murariu, D. Rattat, G. Toubeau, A. Verbruggen, D. Vansthertem, L. Vander Elst and R. N. Muller; "Molecular imaging of alpha $v$ beta3 integrin expression in atherosclerotic plaques with a mimetic of RGD peptide grafted to Gd-DTPA", Cardiovasc Res 2008, 78(1) 148-57.

P. M. Winter, A. M. Morawski, S. D. Caruthers, R. W. Fuhrhop, H. Zhang, T. A. Williams, J. S. Allen, E. K. Lacy, J. D. Robertson, G. M. Lanza and S. A. Wickline; "Molecular imaging of angiogenesis in early-stage atherosclerosis with alpha(v)beta3-integrin-targeted nanoparticles", Circulation 2003, 108(18) 2270-4.

V. Amirbekian, M. J. Lipinski, K. C. Briley-Saebo, S. Amirbekian, J. G. Aguinaldo, D. B. Weinreb, E. Vucic, J. C. Frias, F. Hyafil, V. Mani, E. A. Fisher and Z. A. Fayad; "Detecting and assessing macrophages in vivo to evaluate atherosclerosis noninvasively using molecular MRI", Proc Natl Acad Sci U S A 2007, 104(3) 961-6.

A. Maiseyeu, G. Mihai, T. Kampfrath, O. P. Simonetti, C. K. Sen, S. Roy, S. Rajagopalan and S. Parthasarathy; "Gadolinium containing phosphatidylserine liposomes for molecular imaging of atherosclerosis", J Lipid Res 2008,

W. J. Mulder, G. J. Strijkers, K. C. Briley-Saboe, J. C. Frias, J. G. Aguinaldo, E. Vucic, V. Amirbekian, C. Tang, P. T. Chin, K. Nicolay and Z. A. Fayad; "Molecular imaging of macrophages in atherosclerotic plaques using bimodal PEG-micelles", Magn Reson Med 2007, 58(6) 1164-70.

B. R. Smith, J. Heverhagen, M. Knopp, P. Schmalbrock, J. Shapiro, M. Shiomi, N. I. Moldovan,

M. Ferrari and S. C. Lee; "Localization to atherosclerotic plaque and biodistribution of bio- 
chemically derivatized superparamagnetic iron oxide nanoparticles (SPIONs) contrast particles for magnetic resonance imaging (MRI)", Biomed Microdevices 2007, 9(5) 719-27. E. Lancelot, V. Amirbekian, I. Brigger, J. S. Raynaud, S. Ballet, C. David, O. Rousseaux, S. Le Greneur, M. Port, H. R. Lijnen, P. Bruneval, J. B. Michel, T. Ouimet, B. Roques, S. Amirbekian, F. Hyafil, E. Vucic, J. G. Aguinaldo, C. Corot and Z. A. Fayad; "Evaluation of matrix metalloproteinases in atherosclerosis using a novel noninvasive imaging approach", Arterioscler Thromb Vasc Biol 2008, 28(3) 425-32. C. von zur Muhlen, D. von Elverfeldt, J. A. Moeller, R. P. Choudhury, D. Paul, C. E. Hagemeyer, M. Olschewski, A. Becker, I. Neudorfer, N. Bassler, M. Schwarz, C. Bode and K. Peter; "Magnetic resonance imaging contrast agent targeted toward activated platelets allows in vivo detection of thrombosis and monitoring of thrombolysis", Circulation 2008, 118(3) 258-67. H. Alsaid, G. De Souza, M. C. Bourdillon, F. Chaubet, A. Sulaiman, C. Desbleds-Mansard, L. Chaabane, C. Zahir, E. Lancelot, O. Rousseaux, C. Corot, P. Douek, A. Briguet, D. Letourneur and E. Canet-Soulas; "Biomimetic MRI Contrast Agent for Imaging of Inflammation in Atherosclerotic Plaque of ApoE-/- Mice: A Pilot Study", Invest Radiol 2009, and J. Narula; "Noninvasive detection of plaque instability with use of radiolabeled annexin A5 in patients with carotid-artery atherosclerosis", N Engl J Med 2004, 350(14) 1472-3. 
Chapter 7 


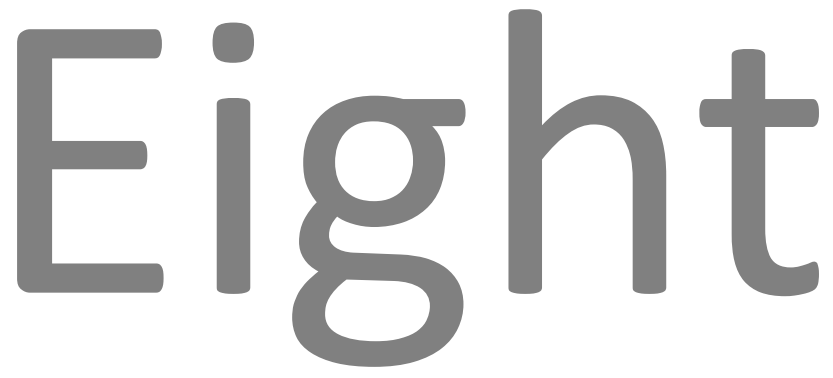

Summary

Samenvatting

Dankwoord

Curriculum Vitae

List of Publications 
Chapter 8 


\section{Summary}

Atherosclerosis is a systemic disease, developing in most humans during aging in which, among others, cholesterol-esters and monocytes are deposited at specific sites in the vascular wall. This causes an increase in vascular wall thickness, which may be remodeled either into the lumen (narrowing the lumen) or outward. Furthermore, inflammation occurs at these sites due to the presence of monocytes and macrophages. Eventually, plaque rupture may occur, causing downstream infarctions. Development of atherosclerosis can be more progressive due to, for example, genetic predisposition, high-fat diet, or smoking. During development of atherosclerotic plaques, not only plaque size increases, but also the molecular and cellular composition changes. Furthermore, there are molecular and cellular differences between stable plaques and plaques at risk of rupture. Visualization of specific pathologic characteristics would allow accurate distinction between non-symptomatic plaques and plaques that are potentially dangerous. In this thesis, the potential of nanoparticles in the molecular imaging of vulnerable plaques is examined.

In chapter 2 basic principles of imaging of murine carotid arteries and atherosclerosis are discussed. First, the cellular composition of vascular walls is explained. Though the development of the disease is similar, there are differences between human and murine atherosclerosis. This is discussed next. Morphological features of vulnerable atherosclerotic plaques are discussed. The physical principles of two-photon laser scanning microscopy (TPLSM) are explained. Finally, practical information on the imaging of atherosclerotic plaques in murine carotid arteries using TPLSM is provided.

Chapter 3 reviews various novel optical nanoparticles for the labeling of vulnerable atherosclerotic plaques. Advantages and disadvantages of quantum dots, and gold, diamond, carbon, and silica nanoparticles are discussed with respect to their optical characteristics and potential toxicity. Furthermore, endoscope-based optical imaging techniques that are in development for in vivo or clinical use are discussed. In the future these techniques may in the future contribute to personalized, specifically targeted treatment of cardiovascular disease. 


\section{Chapter 8}

Chapter $\mathbf{4}$ describes a quantum dot-based nanoparticle that allows imaging of apoptosis and activated platelets. Bimodality of the nanoparticle allows visualization by both fluorescence microscopy (by the quantum dot) and magnetic resonance imaging (MRI) imaging (by the attached Gd-DTPA moieties). This nanoparticle is functionalized by Annexin A5, a ligand that specifically targets phosphatidylserine (PS). PS is exposed on the cellular membrane of apoptotic cells and activated platelets. The functionality of this nanoparticle was shown with both TPLSM and MRI. TPLSM results demonstrated that the nanoparticle specifically bound to PS on the outer cellular membrane of apoptotic Jurkats cells and activated platelets. The nanoparticle also bound to damaged cells in the wire-injured carotid artery, as shown with TPLSM. Longitudinal relaxivity of the bimodal nanoparticle was substantially increased by the use of lysine-backbone Gd-DTPA wedges instead of single Gd-DTPA. This improves the ability of the nanoparticle to be visualized by MRI, as the use of Gd-DTPA wedges amplifies the number of MRI-contrast agents by a factor eight. Indeed, MRI results showed that the same nanoparticle allowed visualization of the three in vitro samples with MRI.

The bimodal quantum dot, described in chapter 4, was utilized for imaging either collagen or cell death in atherosclerotic plaques of carotid arteries of ApoE-/- mice in chapter 5. TPLSM revealed that both types of Qdots bound to hot-spots in intimal as well as deeper layers of the atherosclerotic plaque. This was shown to be specific binding as the control particle, without any targeting ligand, hardly exhibited any labeling of plaques. Likely, quantum dots access vascular targets only at sites showing a certain permeability, or endothelial damage. MRI showed a minor increase in signal intensity for collagen-targeting quantum dots only. Future experiments may reveal whether MRI visualization can be improved and if the relation between the amount of plaque labeling by quantum dots and plaque vulnerability can be determined.

Chapter 6 describes a study in which a novel bimodal $\alpha_{2}$-antiplasmin-based contrast agent was directed against fresh thrombi. During the early phases of thrombus formation, activated factor XIII (FXIIIa) covalently cross-links $\alpha_{2}$-antiplasmin to fibrin, indicating the potential of $\alpha_{2}$-antiplasmin-based contrast agents (CAs) in detecting early thrombus formation. A bimodal CA was synthesized by coupling gadolinium-DTPA and rhodamine to an $\alpha_{2}$-antiplasmin-based peptide. In vitro results showed that the CA 
binds specifically to fibrin in whole-blood clots mediated by FXIIla, as was shown both with MRI and TPLSM. In an in vivo mouse model of thrombus formation the combination of MRI and TPLSM was used to show the applicability of the CA to specifically enhance the signal intensity of fresh thrombi in vivo.

The General Discussion in chapter 7 discusses the requirements for optimized visualization of novel nanoparticles. Three different nanoparticles were used in this thesis. Because of their different composition they exhibit different optical and magnetic resonant properties and they showed different in vivo behavior. For optimized MRI visualization, which has lower sensitivity than TPLSM, four properties should be kept in mind. These are the bioavailability of the target, the affinity of the ligand for the target, the availability of the nanoparticle, and the size of the nanoparticle in case of subendothelial targets. The various characteristics of these nanoparticles are compared and their influence on the outcome of the experiments is discussed. 


\section{Samenvatting}

Aderverkalking is een ziekte die bij bijna iedereen voorkomt, in bepaalde grote bloedvaten door het hele lichaam. Het verloop van de ziekte kan versneld worden door bijvoorbeeld genetische eigenschappen, slechte voeding, of roken. Het is een proces waarbij cholesterol op specifieke plekken in de vaatwand worden gedeponeerd. Deze plekken worden plaques genoemd. Witte bloedcellen (ontstekingscellen) gaan daar actief naar toe om cholesterol te verwijderen. Doordat cholesterol in de vaatwand gedeponeerd blijft worden, kunnen de witte bloedcellen het 'opruimen' van cholesterol niet meer bijhouden en kunnen ze doodgaan. Vervolgens gaat een plaque ontsteken, waarbij er nog meer witte bloedcellen worden aangetrokken vanuit de bloedbaan. Uiteindelijk leidt dit tot de vorming van de 'necrotic core', oftewel een dode kern in de plaque. Door verschillende processen verandert de moleculaire en cellulaire samenstelling van de plaque in de loop van de tijd. Dit kan op den duur leiden tot scheuren van de plaque (plaque-ruptuur). Zo wordt bijvoorbeeld het fibreuse kapsel, een laag bestaande uit voornamelijk collageen die vermoedelijk zorgt voor de stabiliteit van de plaque, dunner door aanwezige enzymen (afkomstig uit onder andere macrofagen). Vele moleculaire en cellulaire processen spelen een rol in plaque-ruptuur; het is nog niet duidelijk welke de belangrijkste is. Bij plaque-ruptuur komt de necrotic core van de plaque in aanraking met de bloedstroom, waardoor bloedstolsels ontstaan en verstoppingen (infarcten) kunnen optreden.

De groei van plaques vindt niet alleen plaats naar binnen toe (bloedvat-vernauwend), maar kan ook naar buiten toe plaats vinden, waarbij het bloedvat niet vernauwd wordt. Dit heeft tot gevolg dat niet alle plaques zichtbaar zijn bij angiografie, waarbij de bloedstroom in de bloedvaten zichtbaar gemaakt wordt. Bovendien zijn er moleculaire en cellulaire verschillen tussen plaques die stabiel zijn en instabiele plaques die mogelijk wel scheuren en daardoor infarcten kunnen veroorzaken. Het is dan ook van belang dat de mogelijk gevaarlijke plaques op een eenduidige manier zichtbaar gemaakt kunnen worden. Wanneer de specifieke moleculaire kenmerken van een instabiele plaque zichtbaar gemaakt kunnen worden zou het dus mogelijk moeten zijn om onderscheid te 
maken tussen de stabiele en instabiele plaques. Het zichtbaar maken van moleculaire kenmerken door nanodeeltjes (deeltjes in de grootte orde van een miljoenste van een millimeter) heet 'Molecular Imaging'. In dit proefschrift is het vermogen van verschillende nanodeeltjes onderzocht om, op basis van molecular imaging, instabiele plaques zichtbaar te maken. Er is gekeken naar drie verschillende plaque-componenten: dode cellen, collageen en geactiveerde bloedplaatjes (die in bloedstolsels voorkomen). Hiervoor zijn, naast in vitro systemen (zoals celculturen) ook in vivo (levende) muismodellen van atherosclerose en bloedstolselvorming gebruikt. Verder zijn twee verschillende beeldvormingstechnieken gebruikt: fluorescentie microscopie vanwege de mogelijkheid om details van cellen te bekijken, en magnetic resonance imaging (MRI) vanwege de klinische toepasbaarheid en de mogelijkheid om van buitenaf bloedvaten te bekijken.

In hoofdstuk 2 wordt eerst de algemene cellulaire samenstelling van de vaatwand behandeld, waarbij aandacht wordt besteed aan een aantal eigenschappen van instabiele plaques. De vorming van aderverkalking in muizen wordt apart besproken, omdat deze nogal verschilt van aderverkalking bij mensen. Vervolgens worden de essentiële elementen van de gebruikte microscopische techniek (twee-foton laser scanning microscopie; TPLSM) besproken. Als laatste worden de praktische kanten met betrekking tot het met TPLSM waarnemen van plaques in muizen-halsslagaders behandeld.

Hoofdstuk $\mathbf{3}$ is een literatuuroverzicht van nieuwe nanodeeltjes die gebruikt kunnen worden bij optische beeldvormingstechnieken, toegepast op instabiele atherosclerotische plaques. De optische kenmerken en mogelijke toxiciteit van quantum dots (dit zijn sterk fluorescerende nanodeeltjes gemaakt van halfgeleider kristallen), en goud-, diamant-, koolstof- en silicadeeltjes worden één voor één besproken. Aangezien microscopie technieken niet eenvoudig klinisch toepasbaar zijn, worden ook mogelijkheden voor klinisch gebruik van optische beeldvormingstechnieken besproken. Endoscopietechnieken, waarbij microscopie mogelijk gemaakt wordt met behulp van optische fibers die een bloedvat ingeschoven kunnen worden, zouden het mogelijk kunnen maken om van binnen uit het bloedvat de vaatwand optisch te bekijken met een hogere resolutie dan met bijvoorbeeld MRI of computed tomography (CT). Deze 


\section{Chapter 8}

technieken kunnen in de toekomst mogelijk bijdragen aan individuele en specifieke behandeling van hart- en vaatziekten.

In hoofdstuk 4 wordt een nanodeeltje beschreven dat als basis uit een quantum dot bestaat. Dit nanodeeltje is specifiek gericht tegen cellen, die apoptose, ofwel geprogrammeerde celdood, ondergaan. Tijdens apoptose is een specifiek molecuul, phosphatidylserine (PS), aan de buitenkant van de cel aanwezig. PS is niet aanwezig aan de buitenkant van gezonde cellen. PS is verder ook zichtbaar aan de buitenkant van geactiveerde bloedplaatjes, die in bloedstolsels voorkomen. Dit nanodeeltje hecht zich aan PS door middel van Annexine A5 dat aan het nanodeeltje gekoppeld is. Dit molecuul zorgt ervoor dat het nanodeeltje specifiek aan apoptotische cellen en geactiveerde bloedplaatjes bindt. Dit nanodeeltje kan zichtbaar gemaakt worden met twee verschillende beeldvormingstechnieken: fluorescentie microscopie en MRI; dit wordt bimodaliteit genoemd. Het nanodeeltje is fluorescerend dankzij de quantum dot. Aan deze quantum dot is gadolinium gekoppeld, dat ervoor zorgt dat het zichtbaar is met MRI. Voor een verdere versterking van de zichtbaarheid met MRI is gebruik gemaakt van een gadolinium-wedge, een acht-armig molecuul dat het aantal gadolinium-ionen verachtvoudigd. Het resulterende nanodeeltje is getest op een celcultuur in apoptose en op bloedstolsels buiten het dier en bekeken met zowel TPLSM als MRI. Om de fysiologische relevantie beter aan te kunnen tonen is ook een beschadigde muizenhalsslagader na operatieve verwijdering gelabeld met dit nanodeeltje. Ook in dit bloedvat was het nanodeeltje zichtbaar met zowel TPLSM als MRI.

Hoofdstuk 5 beschrijft een in vivo studie waarin het nanodeeltje uit hoofdstuk 4 is gebruikt in muizen met aderverkalking. Er zijn twee versies van het nanodeeltje gebruikt: in het eerste geval is het nanodeeltje gekoppeld aan Annexine A5 en bindt het dus aan apoptotische cellen en geactiveerde bloedplaatjes (die beide aanwezig kunnen zijn in instabiele atherosclerotische plaques); in het tweede geval bindt het nanodeeltje aan collageen dankzij het molecuul CNA35. Collageen is aanwezig in zowel stabiele als instabiele plaques. Vermoedelijk is echter collageen in instabiele plaques meer toegankelijk voor het nanodeeltje omdat het endotheel dat erboven ligt meer beschadigd is. TPLSM opnames lieten zien dat beide versies van het nanodeeltje zowel oppervlakkig als in diepere lagen van de plaque binden. Aangezien de nanodeeltjes 
alleen in specifieke 'hot-spots' zichtbaar zijn lijkt het erop dat ze alleen de plaque labelen wanneer het endotheel toegankelijk, ofwel beschadigd genoeg is. Deze mate van labeling door quantum dots zou een maat kunnen zijn voor plaque instabiliteit. MRI resultaten lieten alleen voor het CNA35-nanodeeltje een zwak signaal zien; het Annexine A5-nanodeeltje was niet zichtbaar met MRI. Aanvullend onderzoek moet gedaan worden om zichtbaarheid met MRI te optimaliseren. Ook de relatie tussen de mate van plaque labeling door quantum dots en plaque instabiliteit moet nog verder worden uitgezocht.

In hoofdstuk 6 wordt een ander soort bimodaal nanodeeltje beschreven dat specifiek gericht is op verse bloedstolsels of trombi. Dit is een veel kleiner deeltje, dat $\alpha_{2}$-antiplasmine gebruikt als bindingsfactor. $\alpha_{2}$-antiplasmine is een molecuul dat op een speciale manier aan het bloedstolsel wordt gekoppeld onder invloed van geactiveerd stollingsfactor XIII. Dit gebeurt alleen in de vroege fase van trombusvorming. Het nanodeeltje was bimodaal vanwege de combinatie van het fluorescerende rhodamine en het MRI-zichtbare gadolinium. Tests met bloedstolsels buiten het lichaam laten zien dat het nanodeeltje specifiek bindt aan een onderdeel van bloedstolsels, fibrine. Bovendien laten deze tests zien dat het nanodeeltje zichtbaar is met zowel MRI als met TPLSM. Dit nanodeeltje is ook in vivo getest in een muismodel van acute trombusvorming. Ook hier is aangetoond dat het nanodeeltje gevisualiseerd kan worden specifiek in verse trombi met zowel MRI als TPLSM.

In de algemene discussie in hoofdstuk 7 worden de vereisten voor het optimaal zichtbaar maken van nieuwe nanodeeltjes besproken. Drie verschillende nanodeeltjes zijn gebruikt in dit proefschrift. Omdat deze verschillend zijn samengesteld hebben ze ook verschillende optische en magnetische resonantie eigenschappen. Bovendien vertonen ze ook verschillend gedrag in levende dieren. Omdat MRI visualisatie van gadolinium moeilijker is dan de weergave van fluorescentie met TPLSM, moet visualisatie met MRI geoptimaliseerd worden. Hiervoor zijn in de algemene discussie vier verschillende eigenschappen genoemd. Dit zijn de beschikbaarheid van het doelmolecuul, de sterkte van binding van het ligand aan het doelmolecuul, de beschikbaarheid van het nanodeeltje, en de grootte van het nanodeeltje wanneer het doelmolecuul onder het endotheel ligt. De verschillende eigenschappen van deze 
Chapter 8

nanodeeltjes worden vergeleken en de invloed van deze eigenschappen op het resultaat van de experimenten wordt besproken. 


\section{Dankwoord}

Na ruim vier jaar hard werken ligt het eindelijk voor me: mijn proefschrift. Uiteraard ben ik er erg blij mee, maar ik heb dit niet alleen gedaan. Ik wil graag op deze plek iedereen bedanken die aan dit eindresultaat heeft bijgedragen. Niet alleen de mensen die direct aan het onderzoek hebben bijgedragen, maar ook zij die mij persoonlijk ondersteund hebben. Dit heb ik op een aantal momenten tijdens mijn promotietraject hard nodig gehad. Een aantal mensen wil ik graag in het bijzonder noemen.

Marc, jij was mijn dagelijks begeleider; eerst als co-promotor en sinds kort als promotor. Ik wil je graag bedanken voor alle mogelijkheden die je me hebt gegeven om onderzoek te doen. Je was altijd erg enthousiast en vol met nieuwe ideeën. Dit heeft mij altijd erg gemotiveerd! Ik ben blij dat ik met jou heb mogen werken. Verder kon ik altijd bij je terecht als er iets was. Jij, Eline en de dames zijn ook goeie 'cat-sitters'! Heel erg bedankt voor alles!

Chris, ook jij ben sinds kort 'gepromoveerd' van co-promotor tot promotor. Jouw inbreng en alle nuttige discussies die we gehad hebben stelde ik altijd zeer op prijs. Heel erg bedankt!

Dick, jouw steun en inbreng heb ik altijd zeer gewaardeerd. Langs de zijlijn was je duidelijk aanwezig! Je stond altijd klaar met concrete adviezen. Bedankt voor alles.

Mijn twee-foton collega's wil ik in het bijzonder bedanken voor de leuke tijd die ik daar gehad heb. Kim, Sietze, Remco, collega's van het eerste uur, dankzij jullie zijn het een prachtige en enerverende vier jaar geworden! Wim, je stond altijd voor me klaar, reuzebedankt!

Ook mijn overige biofysica/BMT/fysiologie-collega's wil ik bedanken. Joost, Irene, Koen, Wilco, met jullie was het altijd gezellig, bij Thembi of waar dan ook! Evelien en Liselotte, jullie vrouwelijke versterking was hard nodig in dit toch overwegende mannenbolwerk! Ik ben blij dat ik af en toe bij jullie mijn ei kwijt kon.

Bij de afdeling biochemie zijn er ook een aantal mensen die ik wil bedanken. Allereerst Niko: hoe druk je het ook had, je had altijd een luisterend oor en tijd voor een gezellige 


\section{Chapter 8}

babbel. Bedankt dat je altijd tijd voor me had! Ook de andere mensen op het Annexinelab: Liset, Kristof, Petra, Heidi, Cecile, bedankt! Verder wil ik ook Tilman in het bijzonder bedanken. Ik mocht al sinds mijn allereerste stage in 2002 met je samenwerken, en dat heb ik altijd met veel plezier gedaan!

Met de afdeling radiologie heb ik ook intensief samengewerkt. Robbert-Jan, vele uren hebben we bij de MRI danwel twee-foton microscoop doorgebracht, maar saai werd het nooit! We raakten niet uitgepraat over hoe je jullie (of ik mijn) huis zou moeten inrichten of verbouwen. Of over de aanschaf van auto's, iets waar we het maar niet over eens werden $(-)$. Ik vond het altijd erg gezellig om met jou samen te werken! Ook Eline, Walter en Jos wil ik bedanken voor hun tijd en moeite.

Onmisbaar zijn de mensen geweest die de muis-operaties hebben gedaan. Nicole als eerste; ontelbaar veel canules heb je geplaatst, zonder jou was dit boekje er zeker nooit geweest! Superbedankt, ook voor de gezellige praatjes tijdens de operaties en daarbuiten. Rik, bedankt voor het plaatsen van (eveneens) ontelbaar veel collars! Ook Viviane wil ik graag wil ik bedanken voor haar hulp. Bernard, dankzij jouw techniek om carotiden open te knippen met een mini-schaartje en een paar spelden heb ik mooie opnames kunnen maken met de twee-foton microscoop! Heel erg bedankt voor je tip.

Susanne en Vivian, ik ben erg blij dat jullie er altijd voor me zijn! Heel erg bedankt voor jullie vriendschap en steun, zeker in de afgelopen maanden! Zonder jullie had ik het niet gered.... Jullie zijn schatten! Ook mijn oud-studiegenoten Thijs en Coen wil ik bedanken, ik vind het leuk dat we door de jaren heen contact zijn blijven houden!

Steve, jij hebt een belangrijke rol voor me gespeeld de afgelopen jaren. Ik wil je bedanken voor al je steun door mijn studie- en promotiejaren heen. Han en Peter, jullie stonden altijd voor me klaar. Zeker ook bedankt voor alle wintersportvakanties! Die waren erg gezellig. Marvin, Sharon, Kimberley en Robin, bedankt dat jullie voordeur (nog steeds) open staat! Joey, boefke, ik ben er trots op je peettante te zijn!

Als laatste wil ik mijn familie bedanken, zonder jullie was het niet gelukt! Bart, grote broer, ik ben trots op je. Bedankt voor het maken van de mooie omslag! Anique, ook jij bedankt voor alle gezelligheid. 
Pap en mam, jullie staan werkelijk altijd voor me klaar. Jullie zijn een flinke steun geweest de afgelopen jaren, in het bijzonder de laatste paar maanden. Niet in het minst omdat jullie me voor de derde keer in mijn AIO-tijd helpen met klussen en verhuizen... Heel erg bedankt voor alles! Pap, ik vond het heel fijn dat je verderop op de gang zat en dat ik (bijna) altijd kon binnenvallen, en dat je ook regelmatig even bij mij kwam checken of alles wel goed ging! 


\section{Curriculum Vitae}

Lenneke Prinzen was born on April 1, 1980 in Maastricht, the Netherlands. From 1992 until 1999 she attended secondary education (VWO) at the Trichter College, Maastricht, intermitted by a year (1995-1996), during which she attended Towson High School, Towson, MD, USA. Next, she studied Biomedical Engineering at the Eindhoven University of Technology (TU/e) from 1999-2004. During this time, she performed an internship at the Biochemistry department at Maastricht University, and an externship at the Physiology department at the Ludwig Maximilians Universität, München, Germany; her graduation thesis was done at the department of Molecular Cell Biology, Maastricht University. She received her Master's degree in 2004 with the designation 'cum laude'. Furthermore, she was nominated for the TU/e 2005 Mignot Graduation Award. In 2005 she started as a PhD-student at the department of Biophysics (now Biomedical Engineering) at Maastricht University on the subject of 'Quantum dots in ischemic diseases', which was part of the SenterNovem/BSIK project on 'Molecular Imaging of Ischemic Heart Disease'. During this time she attended several CARIM courses as well as general education courses. She also attended a number of international conferences, such as the Joint Molecular Imaging Conference and the conference organized by the International Society on Magnetic Resonance in Medicine. She received three travel awards for these conferences. As of May 1, 2009, she is working as a Post-Doc at the department of Biomedical Engineering at the Maastricht University. 


\section{List of publications}

\section{Articles}

L. Prinzen, R. J. Miserus, A. Dirksen, T. M. Hackeng, N. Deckers, N. J. Bitsch, R. T. Megens, K. Douma, J. W. Heemskerk, M. E. Kooi, P. M. Frederik, D. W. Slaaf, M. A. Zandvoort and C. P. Reutelingsperger; "Optical and Magnetic Resonance Imaging of Cell Death and Platelet Activation Using Annexin A5-Functionalized Quantum Dots", Nano Lett 2007, 7(1) 93-100.

K. Douma*, L. Prinzen*, D. Slaaf, C. P. M. Reutelingsperger, E. A. L. Biessen, T. M. Hackeng, M. J. Post and M. A. M. J. van Zandvoort; "Nanoparticles for Optical Molecular Imaging of Atherosclerosis", Small 2009, 5(5) 544-557.

L. Prinzen, R. J. J. H. M. Miserus, N. Bitsch, T. M. Hackeng, M. E. Kooi, D. W. Slaaf, C. P. M. Reutelingsperger and M. A. M. J. Zandvoort; "Hot-spot labeling of apoptosis and collagen in atherosclerotic plaques using bimodal quantum dots", in preparation.

R. J. J. H. M. Miserus, M. V. Herias*, L. Prinzen*, M. B. I. Lobbes, R. J. van Suylen, A. Dirksen, T. M. Hackeng, J. W. M. Heemskerk, J. M. A. van Engelshoven, M. J. A. P. Daemen, M. A. M. J. van Zandvoort, S. Heeneman and M. E. Kooi; "Molecular Imaging of Early Thrombus Formation using a Bimodal $\alpha_{2}$-antiplasmin-based contrast agent", JACC Cardiovasc Imaging 2009, in press.

R. T. A. Megens, S. Reitsma, L. Prinzen, M. G. A. oude Egbrink, W. Engels, P. J. A. Leenders, E. J. L. Brunenberg, K. D. Reesink, B. J. A. Janssen, B. M. ter Haar Romeny, D. W. Slaaf and M. A. M. J. Zandvoort; "In vivo high resolution structural imaging of large arteries in small rodents using two-photon laser scanning microscopy", Journal of Biomedical Optics 2009, in press.

K. Douma, R. T. Megens, S. Reitsma, L. Prinzen, D. W. Slaaf and M. A. Van Zandvoort; "Two-photon lifetime imaging of fluorescent probes in intact blood vessels: a window to sub-cellular structural information and binding status", Microsc Res Tech 2007, 70(5) 467-75. 


\section{Chapter 8}

M. J. Kuijpers, K. Gilio, S. Reitsma, R. Nergiz-Unal, L. Prinzen, S. Heeneman, E. Lutgens, M. A. M. J. van Zandvoort, B. Nieswandt, M. G. Oude Egbrink and J. W. Heemskerk; "Complementary roles of platelets and coagulation in thrombus formation on plaques acutely ruptured by targeted ultrasound treatment: a novel intravital model", J Thromb Haemost 2009, 7(1) 152-61.

* Authors contributed equally.

\section{Abstracts}

L. Prinzen, N. M. Deckers, W. Engels, D. W. Slaaf, M. A. M. J. Zandvoort and C. Reutelingsperger; "Visualizing instable atherosclerotic plaques using quantum dots (QDs)." Molecular Imaging 2005, 4(3) 278.

L. Prinzen, R. J. J. H. M. Miserus, A. Dirksen, T. M. Hackeng, W. H. Backes, M. E. Kooi, J. W. M. Heemskerk, D. W. Slaaf, C. P. M. Reutelingsperger and M. A. M. J. van Zandvoort; "Towards the imaging of vulnerable atherosclerotic plaques using quantum dots in optical and MR imaging", J Vasc Res 2006, 43(Suppl. 1) 39.

L. Prinzen, R. J. J. H. M. Miserus, A. Dirksen, R. T. Megens, K. Douma, T. M. Hackeng, J. W. Heemskerk, E. Kooi, D. W. Slaaf, C. P. Reutelingsperger and M. A. van Zandvoort; "Towards optical and MR imaging of vulnerable atherosclerotic plaques using bimodal quantum dots", Proc. Intl. Soc. Mag. Reson. Med. 2007, 15(3410).

L. Prinzen, R. J. J. H. M. Miserus, R. T. A. Megens, A. Dirksen, T. M. Hackeng, M. Merkx, E. Kooi, D. W. Slaaf, C. P. M. Reutelingsperger and M. A. M. J. Zandvoort; "Magnetic resonance and fluorescence imaging of vulnerable plaques in mice using bimodal quantum dots", Joint Molecular Imaging Conference 2007, Providence, RI, USA.

R. J. J. H. M. Miserus, L. Prinzen, V. Herias, T. M. Hackeng, A. Dirksen, W. Adriaens, M. A. M. J. Zandvoort, M. Lobbes, M. J. Daemen, J. v. Engelshoven, S. Heeneman and E. Kooi; "In vivo molecular imaging of thrombus formation using a bimodal alpha2-antiplasminbased contrast agent", Joint Molecular Imaging Conference 2007, Providence, RI, USA. 


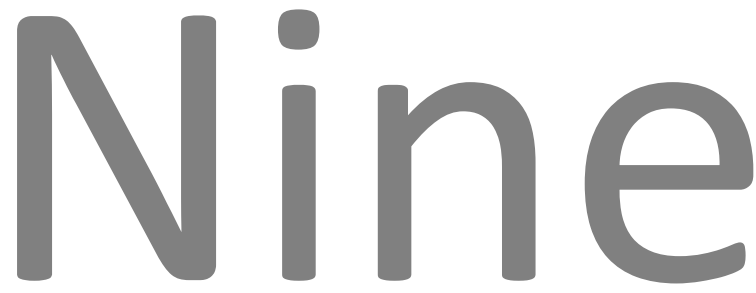

Full color figures 


\section{Chapter 9}

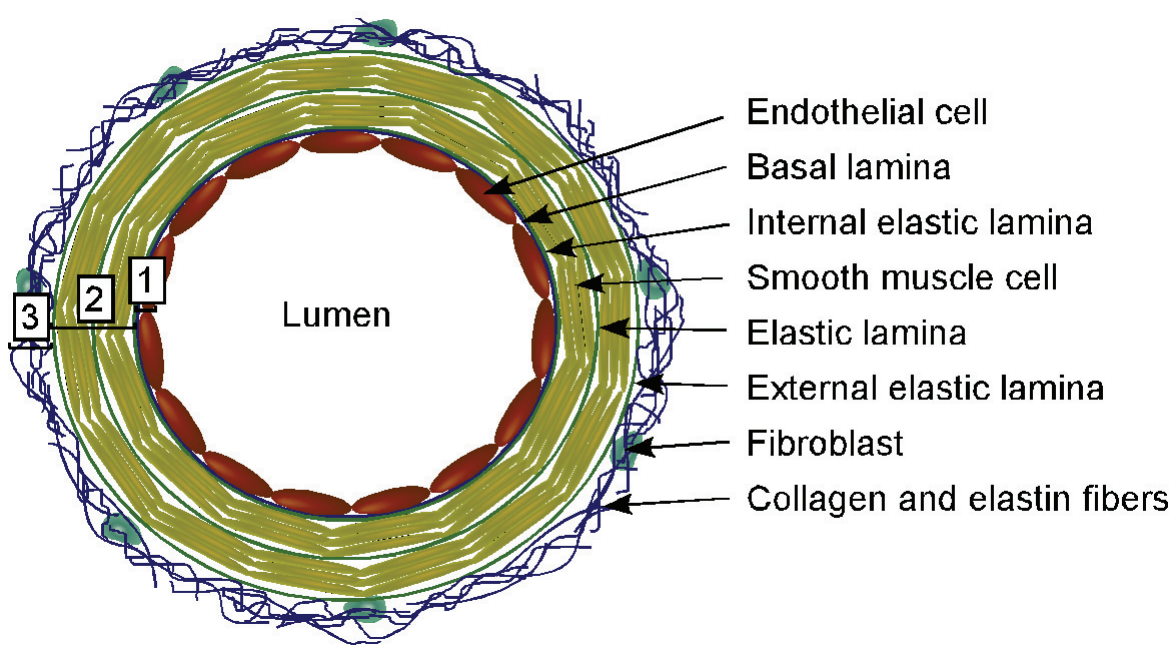

Chapter 2 - figure 2. Schematic representation of transverse view of arterial wall of a murine carotid artery. ' 1 ' indicates tunica intima, ' 2 ' tunica media, and ' 3 ' tunica adventitia.

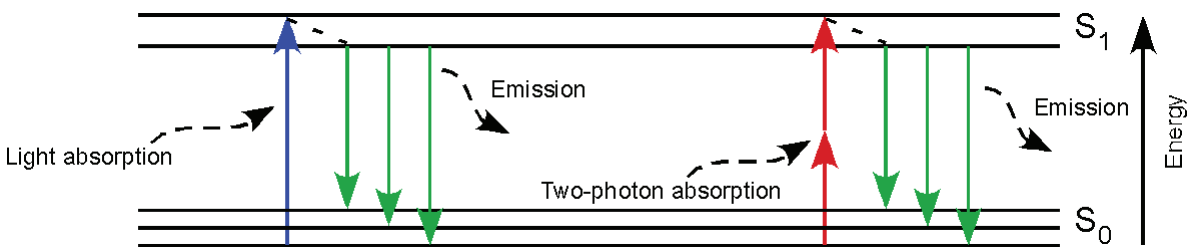

Chapter 2 - figure 2. Simplified Jablonski diagram showing single (blue) and two (red) -photon excitation. 

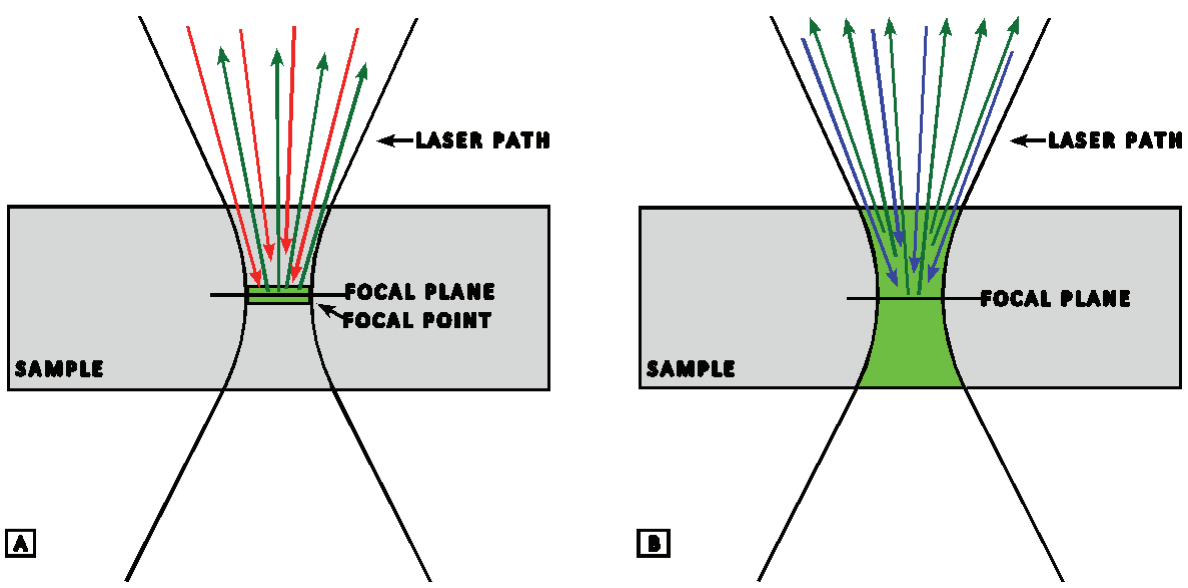

Chapter 2 - figure 4. Schematic representation of excitation and fluorescence emission in TPLSM (A) and confocal microscopy (B). In TPLSM, near infrared laser light (indicated by red arrows) is used to excite molecules in the focal point. As excitation only occurs in the focal point, all of the green emitted light (indicated by green arrows) originates from the focal point, and can be used for reconstruction of the image. In confocal microscopy, blue/green laser light is used for excitation, as indicated by blue arrows. Molecules are excited along the laser path, not necessarily only in the focal plane.
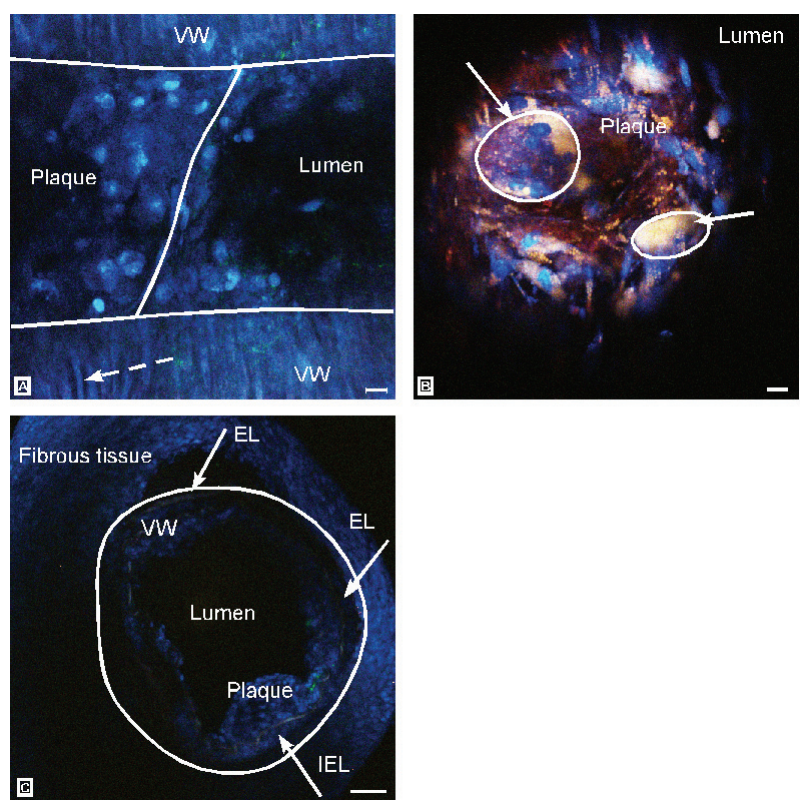

Chapter 2 - figure 6. TPLSM images of plaque-containing carotid arteries with three different methods. A) Artery mounted in flow chamber. In the vascular wall, smooth muscle cells are visible; an example is indicated by dashed arrow. Also inside the plaque cells are visible. Endothelial cells are not visible in this image. B) Artery longitudinally dissected and imaged while in agarose gel, with the luminal side on top. Here, only the plaque is visible. Inside the plaque, foam cells containing multiple nuclei (indicated by circles) are visible. C) Artery transversally dissected. Circle indicates border between vascular wall and surrounding tissue. IEL indicates border between vascular wall and plaque (endothelium is not clearly visible in this image). The three elastic laminae are indicated by arrows. Blue: nuclei; red in B: lipids. VW: vascular wall, EL: elastic lamina, IEL: internal elastic lamina. In A and B, bar $=10 \mu \mathrm{m}$; in $C$, bar $=50 \mu \mathrm{m}$. 


\section{Chapter 9}
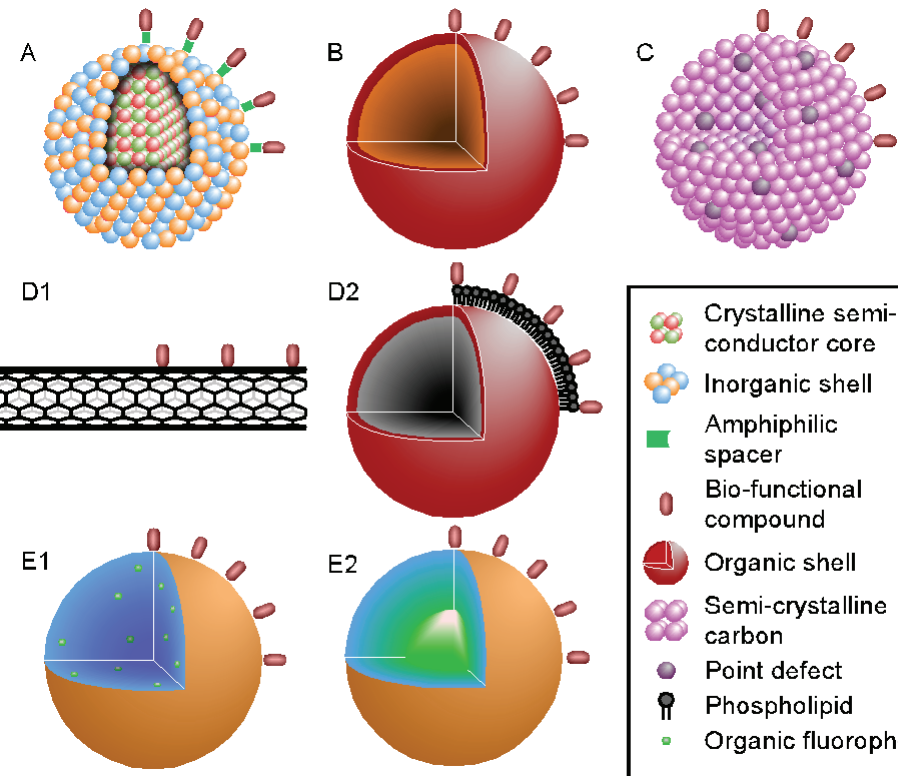

Crystalline semiconductor core

Inorganic shell

Amphiphilic spacer

Bio-functional compound

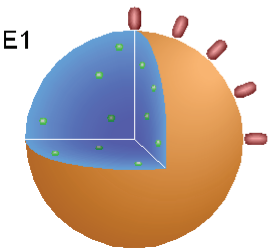

Organic shell

Semi-crystalline carbon

Point defect

if Phospholipid

- Organic fluorophore

Chapter 3 - figure 1. Schematic overview of the composition of A) quantum dot, B) gold nanoparticle, C) diamond nanoparticle, D1) carbon nanotube, D2) carbon dot (C-Dot), E1) silica nanoparticle with encapsulated organic fluorophores, E2) silica nanoparticle with fluorescent core. Several chemical methods for surface binding of bioactive compounds are mentioned in the text. See Table 2 for spatial dimensions.

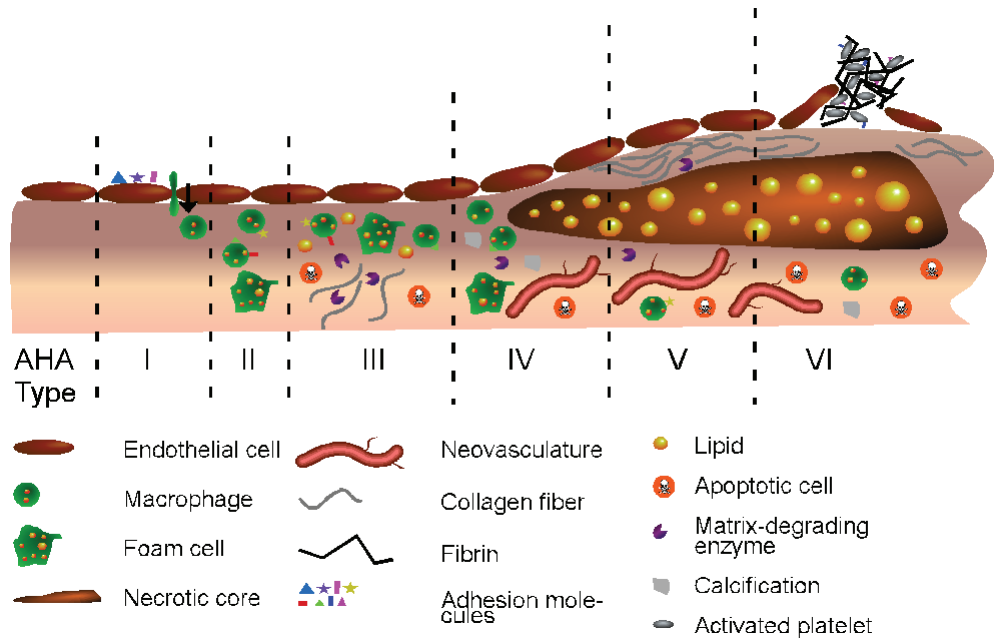

Chapter 3 - figure 2. Schematic representation of human plaque progression. The different types of plaque morphologies are not necessarily successive and not all plaques progress to ruptured plaques (AHA type VI). 


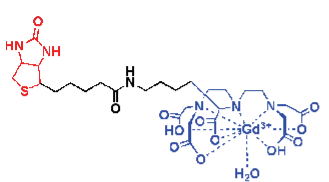

A

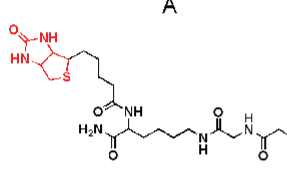

B
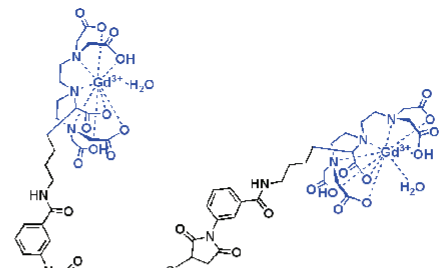


\section{Chapter 9}
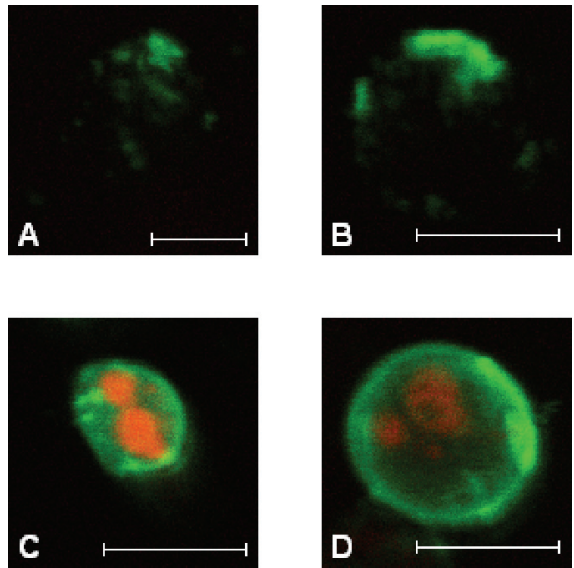

Chapter 4 - figure 4. TPLSM images showing cellular AnxA5-QD525 (green) distribution. All cells were counterstained with PI (red) to determine membrane leakage, i.e., determine the stage of apoptosis. Top-row: early apoptotic Jurkat cells labeled with A; AnxA5-QD-Gd and B; AnxA5-QD-Gd-wedge. Bottom-row: late apoptotic cells labeled with C; AnxA5-QD-Gd and D; AnxA5-QD-Gd-wedge. Bar $=10 \mu \mathrm{m}$.
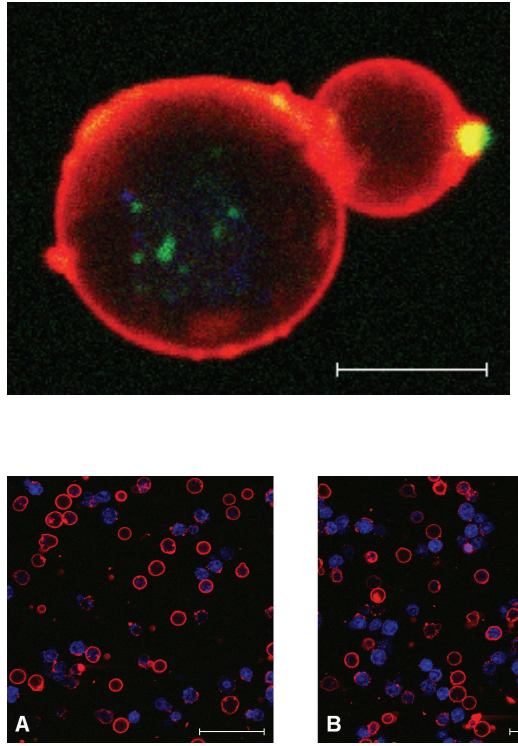
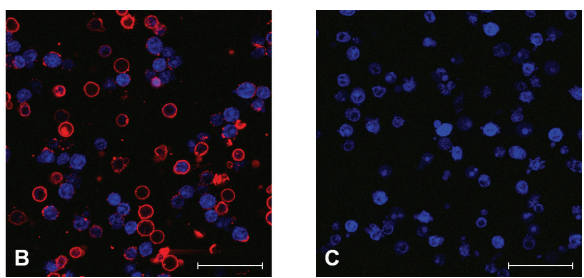

Chapter 4 - figure 6. TPLSM images showing the cellular distributions of QD585 (red) coupled to AnxA5 or a mutant of AnxA5, unable to bind to PS (M1234), on apoptotic Jurkat cells. All cells were counterstained with Syto41 (blue), a nucleic acid stain. A: AnxA5-QD-Gd. B: AnxA5-QD-Gd-wedge. C: M1234-QD-Gd-wedge. Bar = $50 \mu \mathrm{m}$. 

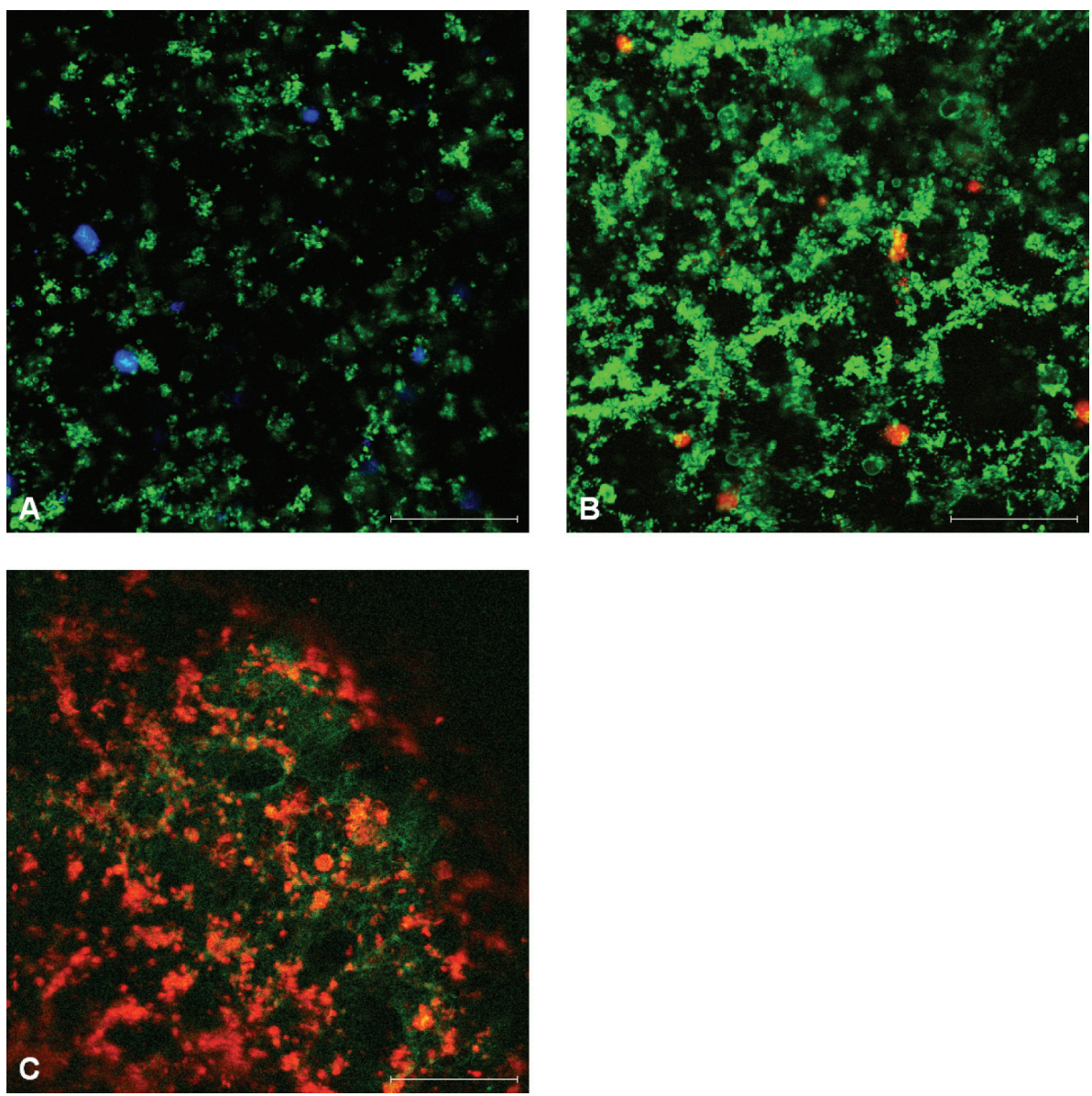

Chapter 4 - figure 9: TPLSM images of whole-blood clots incubated with AnxA5 after clot-formation. A; green: AnxA5-FITC, blue: syto41, a nucleic acid stain; B; green: AnxA5-QD525-Gd, red: acridine red, a cytoplasmatic stain. C; red: AnxA5-QD585-Gd, green: anti-fibrinogen-FITC. Platelets show a bright cellular membrane, similar to apoptotic cells (compare to figures 4/5/6). Bar $=50 \mu \mathrm{m}$. 

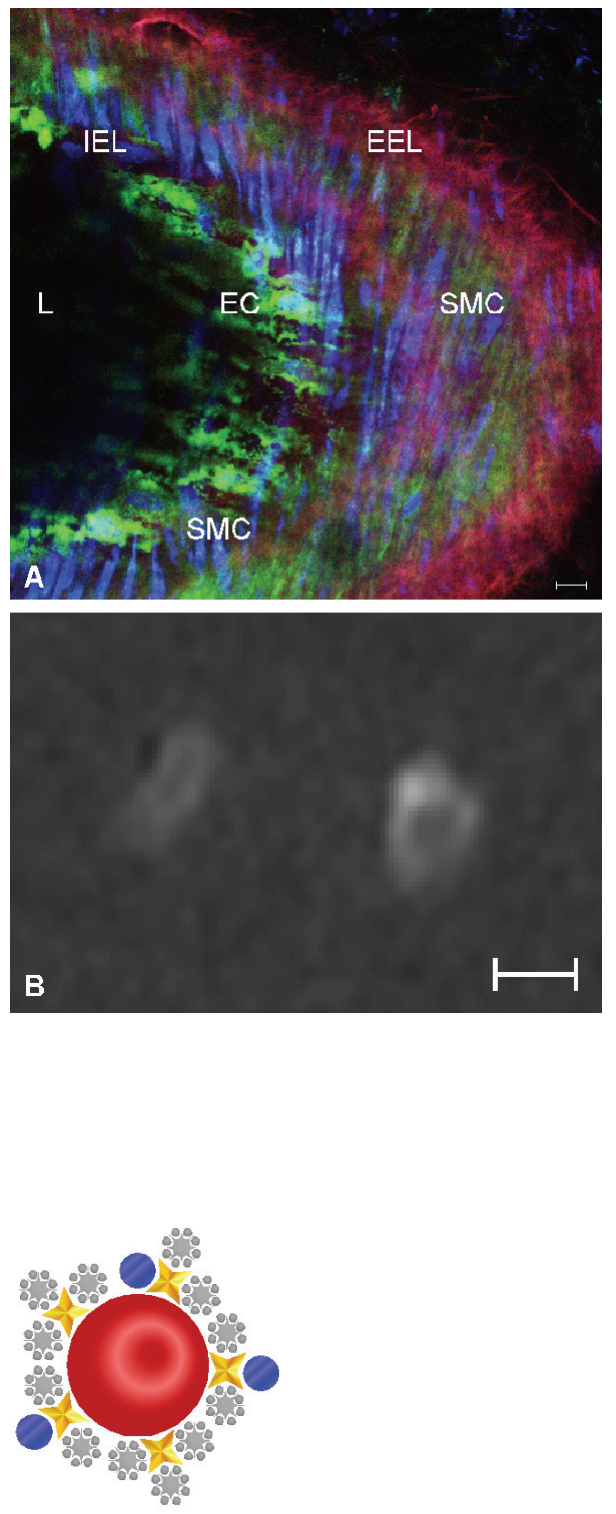

Chapter 4 - figure 10: A; TPLSM image of a wire-injured murine carotid artery, showing the various vascular wall layers. The tunica intima consists of endothelial cells (ECs); the tunica media mainly consists of smooth muscle cells (SMCs). Uptake of nanoparticles is visible in ECs and SMCs. Green: AnxA5-QD-Gd-wedge; red: eosin, labeling elastin laminae; blue, syto41, labeling cell nuclei. L: lumen; IEL: internal elastic lamina; EEL: external elastic lamina. Bar $=10 \mu \mathrm{m}$. The imaging plane was slightly oblique, as opposed to a longitudinal direction of the artery. $B$; transversal MR image of two murine carotid arteries: undamaged (control, left) and damaged (right, same artery as shown in A). Bar = $0.5 \mathrm{~mm}$.
Chapter 5 - figure 1. Schematic representation of Anxor CNA-Qdot. Red: Qdot; yellow: streptavidin; grey: Gd-DTPA8 wedge; blue: Anx or CNA. 


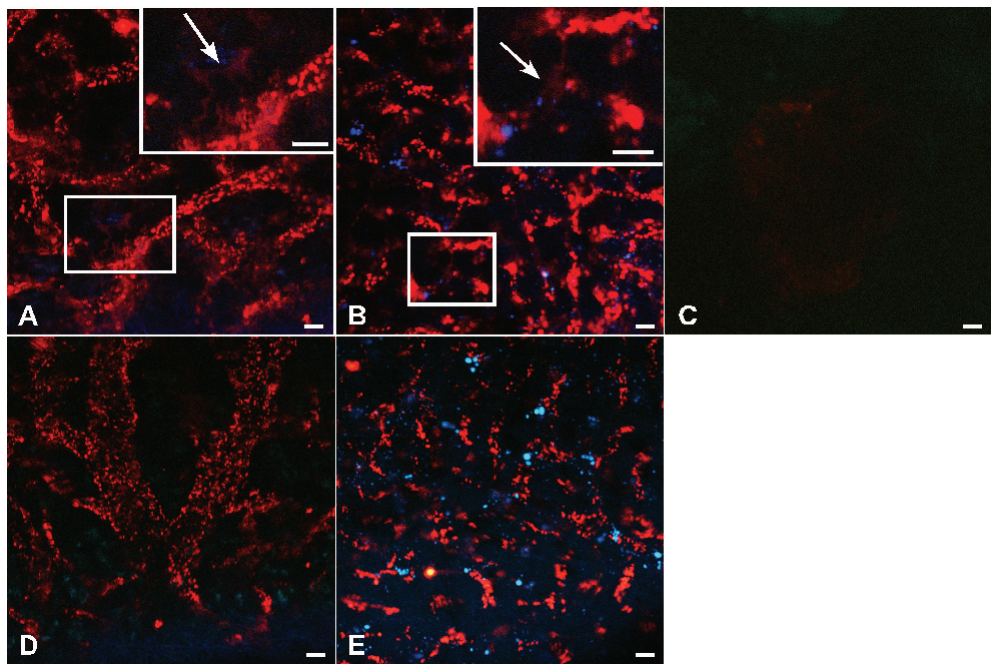

Chapter 5 - figure 2. Distribution of CNA-Qdots in A) spleen, inset is magnification of box; B) liver, inset is magnification of box; C) kidney glomerulus. Distribution of Anx-Qdots in D) spleen and E) liver. Qdots (red), autofluorescence (blue). Arrows in A) and B) indicate collagen-binding Qdots. Bar $=10 \mu \mathrm{m}$.
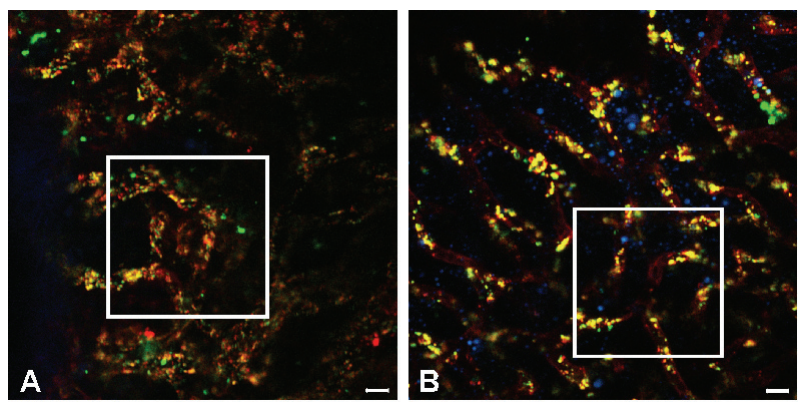

Chapter 5 - figure 3. Difference in distribution of bare Qdots (green) and CNA-Qdots (red). A) spleen, B) liver, C) magnification of boxed part in A), D) magnification of boxed part in B). Arrows indicate collagenspecific binding of CNA-Qdots. Bar $=10 \mu \mathrm{m}$.
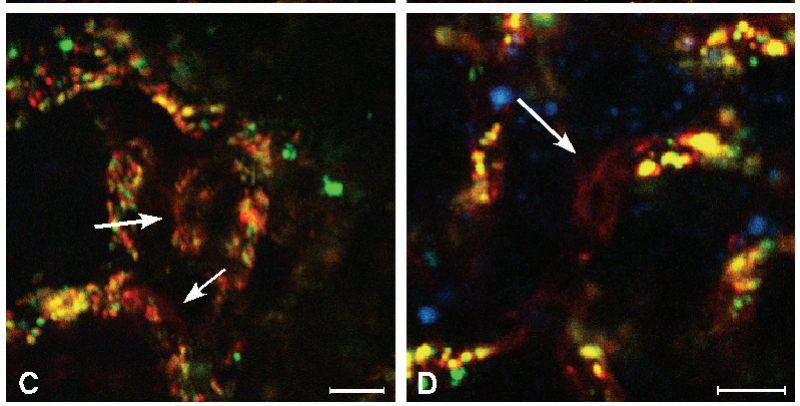


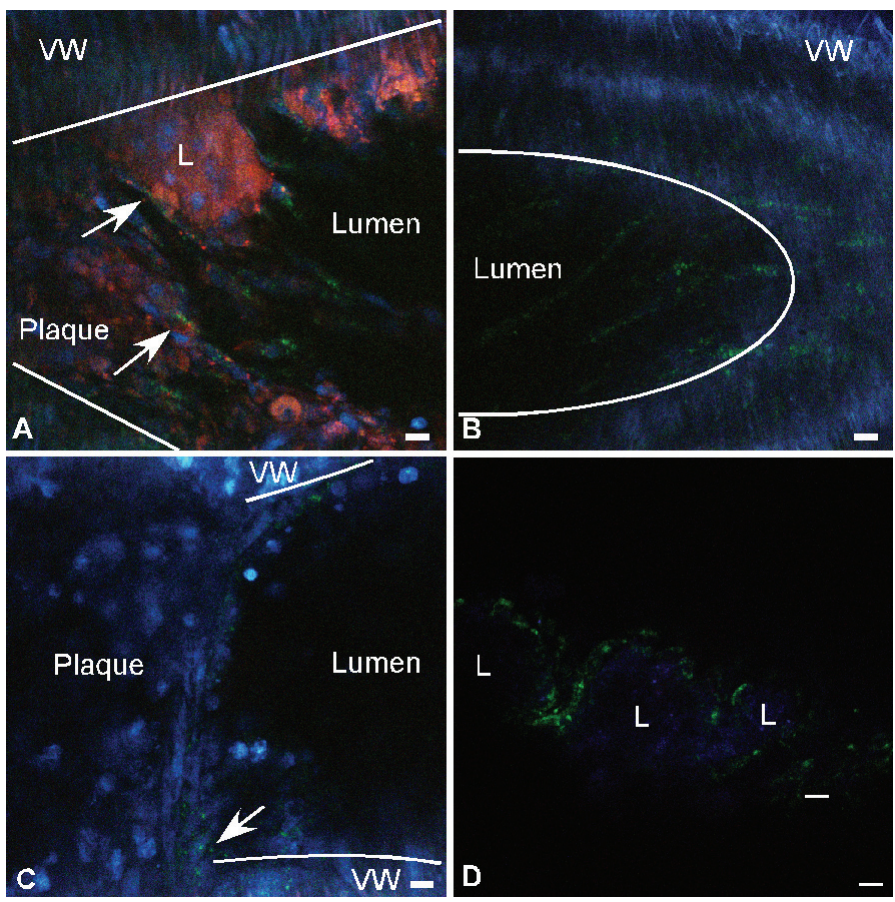

Chapter 5 - figure 6. A and B) Control-Qdots (green) in three-week collar plaques. Both images show carotid arteries in a flow chamber and under $80 \mathrm{mmHg}$ transmural pressure. A) Control-Qdots are bound to endothelium covering lipid pools (type 2). B) Control-Qdots are seen in the grooves of elastic laminae in an area adjacent to plaque (type 2). C and D) Control-Qdots (green) in six-week collar plaques. C) Mounted carotid artery under $80 \mathrm{mmHg}$ (type 2). Endothelial cells on plaque are labeled with control-Qdots, indicated by arrow. D) 'En face' view of carotid artery (type 4). White lines indicate border between media and intima layers. Arrows indicate control-Qdots bound to endothelium. Blue in A) and C) are nucleic acids, and in B) and D) autofluorescence. Red is lipid. ' $\mathrm{L}$ ' is lipid pool, 'VW' is vascular wall. Bar $=10 \mu \mathrm{m}$.

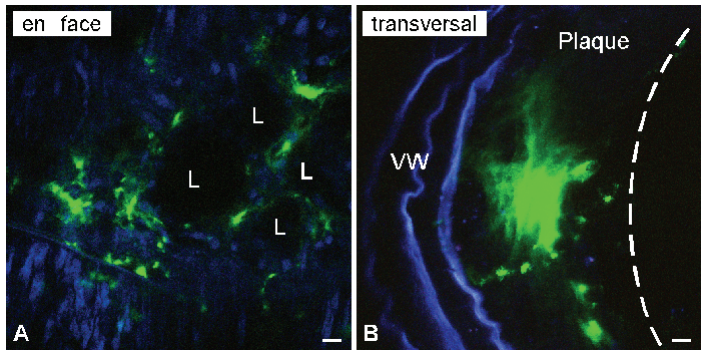

Chapter 5 - figure 7. CNA-Qdots (green) in three-week collar plaques. A) 'En face' view (type 1). B) Transverse view (type 4). Dashed line indicates border between plaque and lumen. In A) blue are nucleic acids, in B) autofluorescence. B) shows the sum of a small stack of images. ' $L$ ' is lipid core, ' $\mathrm{VW}$ ' is vascular wall. Bar $=10 \mu \mathrm{m}$. 


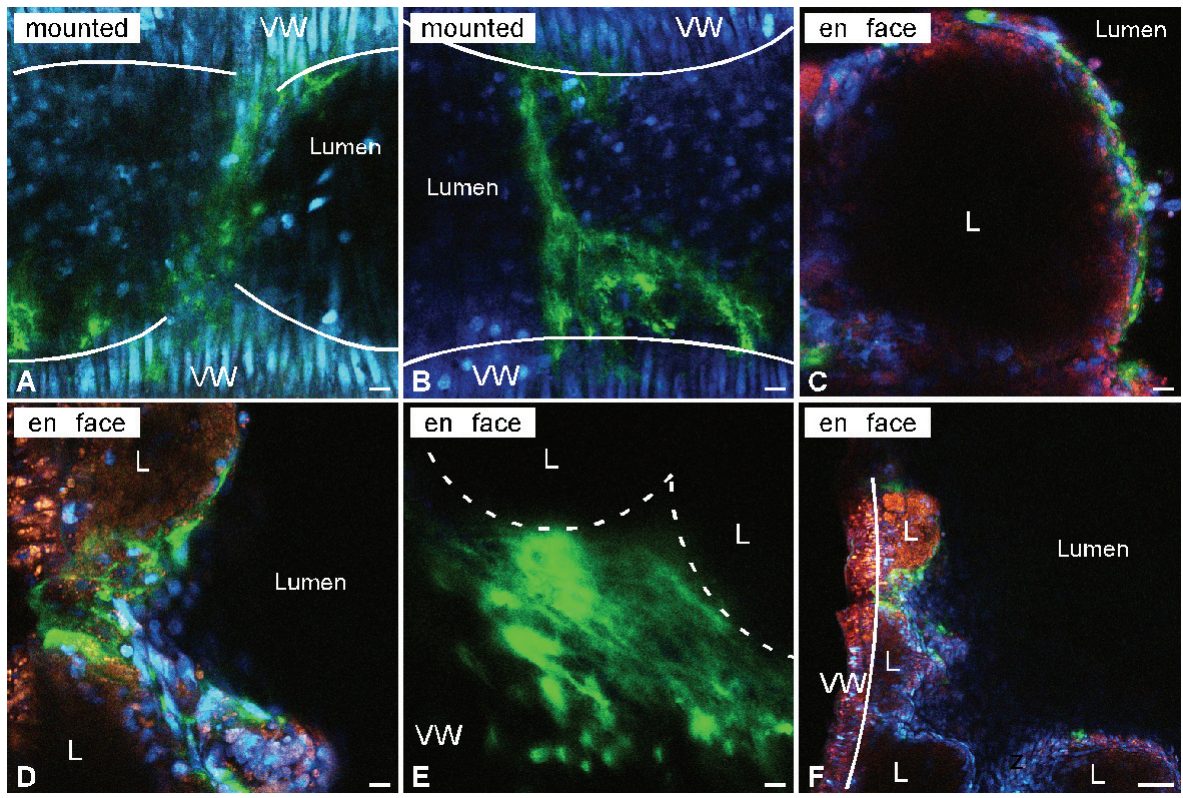

Chapter 5 - figure 8. CNA-Qdots (green) in six-week collar plaques. A) and B) Mounted artery under $80 \mathrm{mmHg}$ transmural pressure, showing fibrous caps labeled with CNA-Qdots (same artery, type 3). C) 'En face' view, showing a lipid pool with CNA-Qdots just underneath the endothelium (type 3). D) 'En face' view, collagen network visible in between two lipid pools (type 3). E) 'En face' view, collagen network visible in between two lipid pools (type 4). F) 'En face' view showing multiple lipid pools, but only one (same as in D) shows bound CNA-Qdots. Blue are nucleic acids, red is lipid. White lines indicate border between media and intima, dotted lines in E) indicate border of lipid pools. ' $\mathrm{L}$ ' is lipid pool, ' $\mathrm{VW}$ ' is vascular wall. Bar $=10 \mu \mathrm{m}$, except in F), bar = $50 \mu \mathrm{m}$.

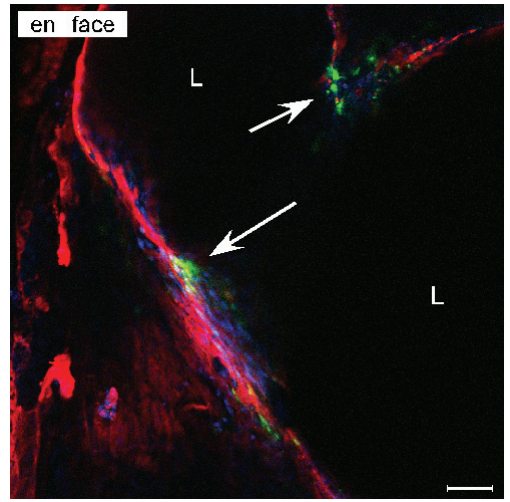

Chapter 5 - figure 9. Two-photon image showing 'en face' view of a plaque, in vivo injected with CNA-Qdot (green) and ex vivo labeled with CNA-streptavidin-Alexa568 (red). Ex vivo labeling resulted in abundant collagen labeling, revealing that CNA-Qdots indeed only label accessible hot-spots of collagen within the plaque. Blue is syto44. Bar $=50 \mu \mathrm{m}$. 

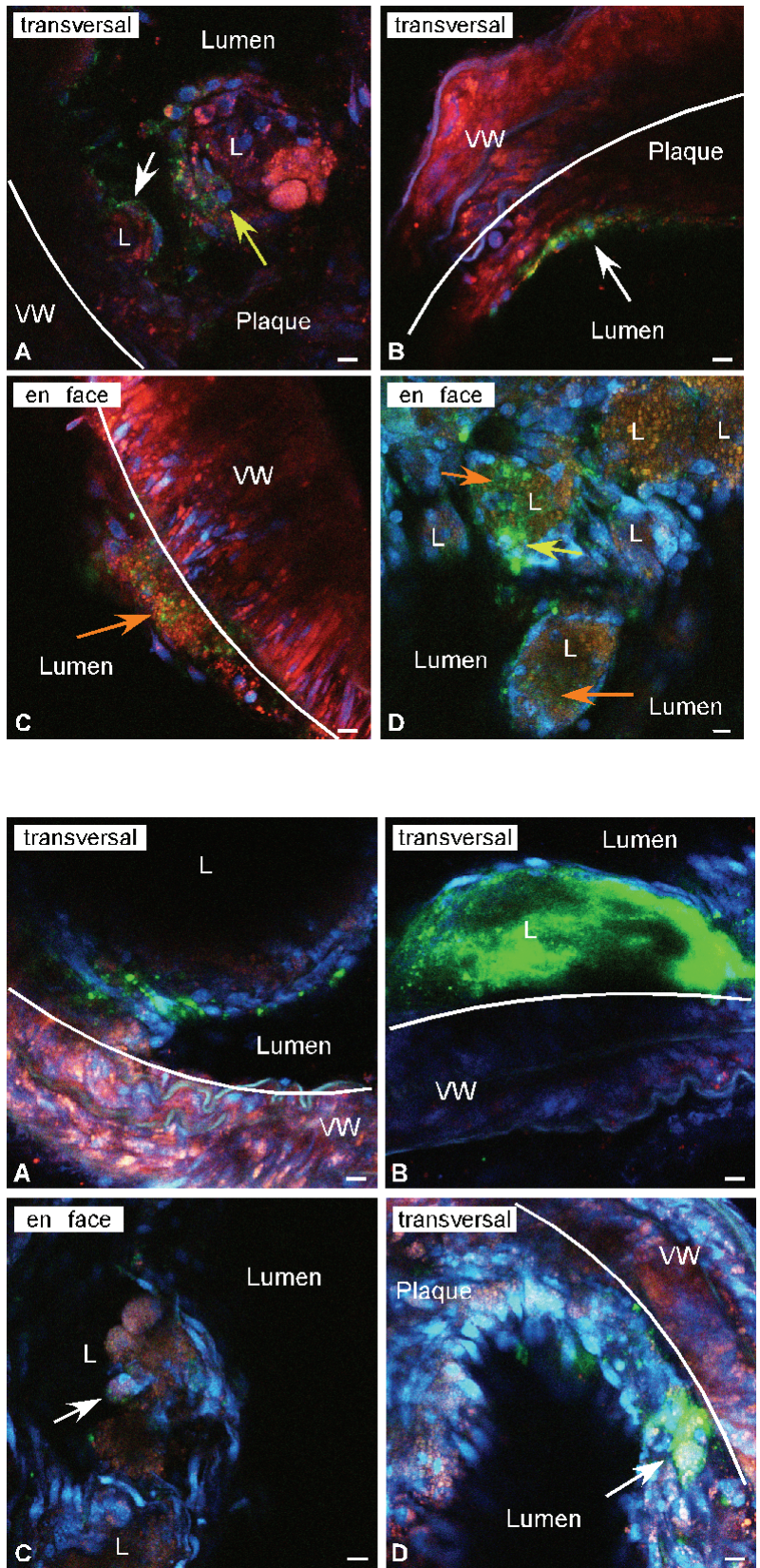

Chapter 5 - figure 10. Anx-Qdots (green) in three-week collar plaques. A) Transverse view of plaque (type 3). B) Transverse view of plaque (type 3). C) 'En face' view of plaque (type 1). Vascular wall is visible because the cutting surface curled up. D) 'En face' view of plaque (type 1). White lines indicate border between media and intima. White arrows indicate endothelial labeling by Anx-Qdots, yellow arrows indicate cellular Anx-Qdots, and orange arrows indicate vesicular Anx-Qdots. ' $L$ ' indicates lipid pool, ' $V W$ ' indicates vascular wall. Red/orange is lipid; blue are nucleic acids. Bar $=10 \mu \mathrm{m}$.

Chapter 5 - figure 11. Anx-Qdots (green) in six-week collar plaques. A) Transverse slice of carotid artery. Anx-Qdots are visible on the endothelium covering the lipid pool (type 4). B) Transverse slice of carotid artery, showing a high load of Anx-Qdots inside a lipid pool (type 4). C) 'En face' view of carotid artery (type 2). Anx-Qdots are visible in a foam cell within a lipid pool. D) Transverse view of plaque in carotid artery (type 4). Arrows indicate (foam) cell that had taken up AnxQdots. White lines indicate border between media and intima. ' $\mathrm{L}$ ' is lipid pool; ' $V W$ ' is vascular wall. Blue are nucleic acids, red is lipid. Bar $=10 \mu \mathrm{m}$. 

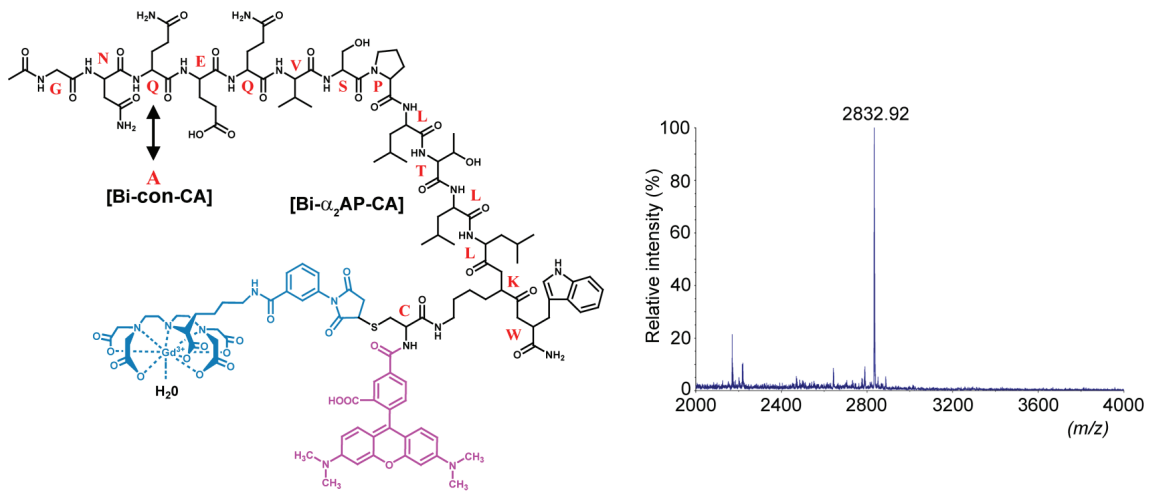

Chapter 6 - figure 1: Schematic representation of the bimodal $\alpha_{2}$-AP-based peptide labeled with rhodamine and DTPA $\left(\mathrm{Bi}-\alpha_{2} \mathrm{AP}-\mathrm{CA}\right)$ and the corresponding mass spectra. MALDI-MS shows a molecular mass of $2832.92 \mathrm{~g} / \mathrm{mol}$ for $\mathrm{Bi}-\alpha_{2} \mathrm{AP}-\mathrm{CA}$. Substitution of one amino acid $\left(Q_{3} \rightarrow A 3\right)$ results in a bimodal control $C A$ (Bi-con-CA).

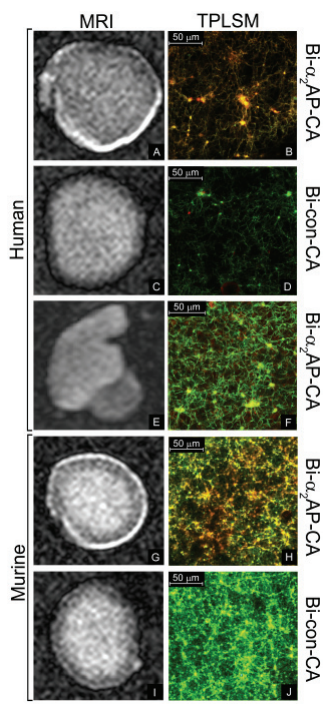

Chapter 6 - figure 2: Two-photon laser-scanning microscopy (TPLSM) and magnetic resonance imaging (MRI) results for human (A-F) and murine (G-J) thrombi incubated with the bimodal $\alpha_{2}$-AP-based contrast agent ( $B i-\alpha_{2} A P$ CA) (A, B, E-H) and the bimodal control CA (Bi-con-CA) (C, D, I, and J). For TPLSM, red color originated from the rhodamine-labeled CAs. Green color indicated the fibrin network due to use of fibrinogen-Oregon green 488 . Yellow color illustrated co-localization of red and green. Hyperintense MR signals were found the edge of thrombi incubated with $B i-\alpha_{2} A P-C A(A, G)$. Lower MR signal intensities were found at the edge of a thrombus exposed to dansylcadaverine and Bi- $\alpha_{2} \mathrm{AP}-\mathrm{CA}(\mathrm{E})$. 

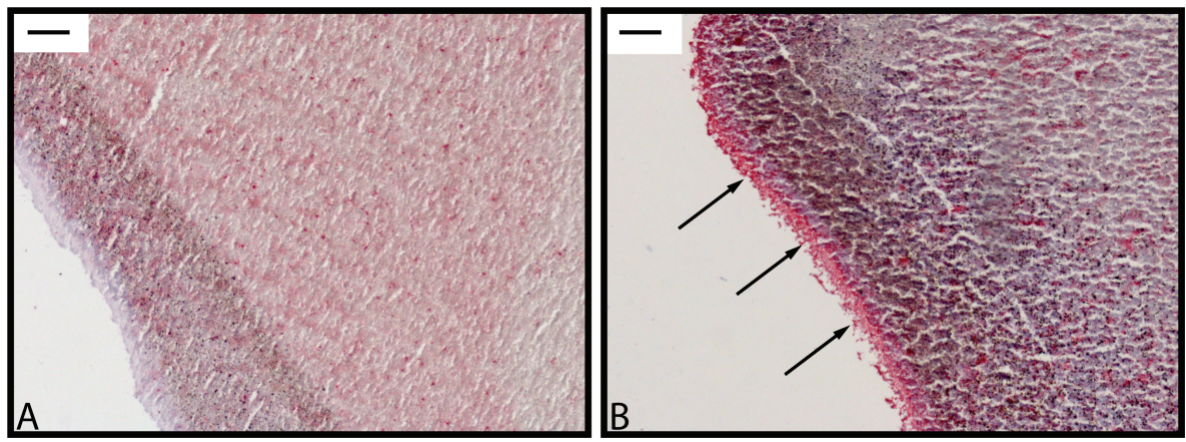

Chapter 6 - figure 3: Immunohistochemical stainings of thrombi. A) factor XIII (FXIII) staining, B) $\alpha_{2}$-antiplasmin ( $\alpha_{2}$-AP) staining. Positive staining is depicted in red/pink color. FXIII staining (scanty spots) was present throughout the whole thrombus while $\alpha_{2}$-AP staining was mainly depicted at the edge of the thrombus (arrows). Bars: $50 \mu \mathrm{m}$.

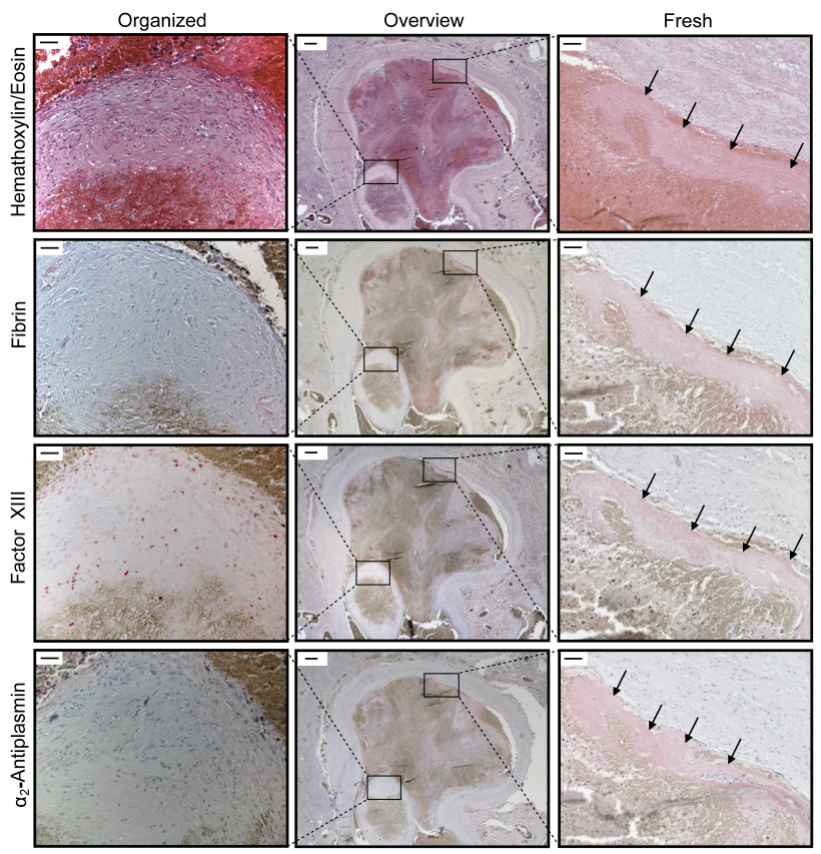

Chapter 6 - figure 4: Lung emboli sections. Middle panels: overview of lung emboli, from top to bottom: Hemathoxyline/Eosin stained, immunohistochemically stained for fibrin, factor XIII, and $\alpha_{2}$-antiplasmin. Bars: $300 \mu \mathrm{m}$. Left panels: enlargements of boxed areas indicating an organized part of the lung emboli. In the enlarged FXIII stained area clear cellular FXIII staining is visable. Bars: 50 $\mu \mathrm{m}$. Right panels: enlargements of boxed areas indicating a fresh segment (arrows). Bars: $50 \mu \mathrm{m}$. 

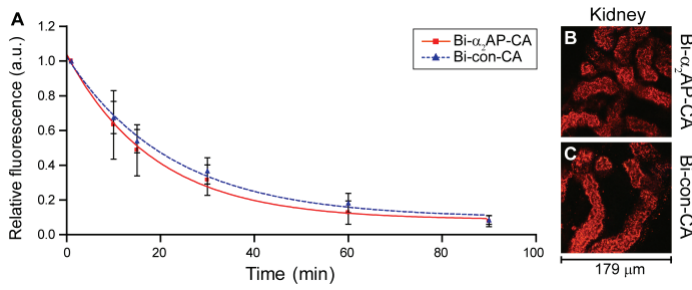

Chapter 6 - figure 5: Clearance and kidney uptake of $\mathrm{Bi}$ $\alpha_{2} \mathrm{AP}-\mathrm{CA}$ and $\mathrm{Bi}-\mathrm{con}-\mathrm{CA}$. Relative fluorescence measo ured in blood samples decreases with time after con$\rightarrow$ trast administration (A). Half-life of the CAs is $14.1 \pm 8.4$ and 16.3 \pm 4.0 minutes for $\mathrm{Bi}-\alpha_{2} \mathrm{AP}-\mathrm{CA}$ and $\mathrm{Bi}$-con-CA, respectively. Both $C A s$ are mainly cleared through the kidney (B-C). Panels B-C: Red: Bimodal CA present in kidney tubuli.

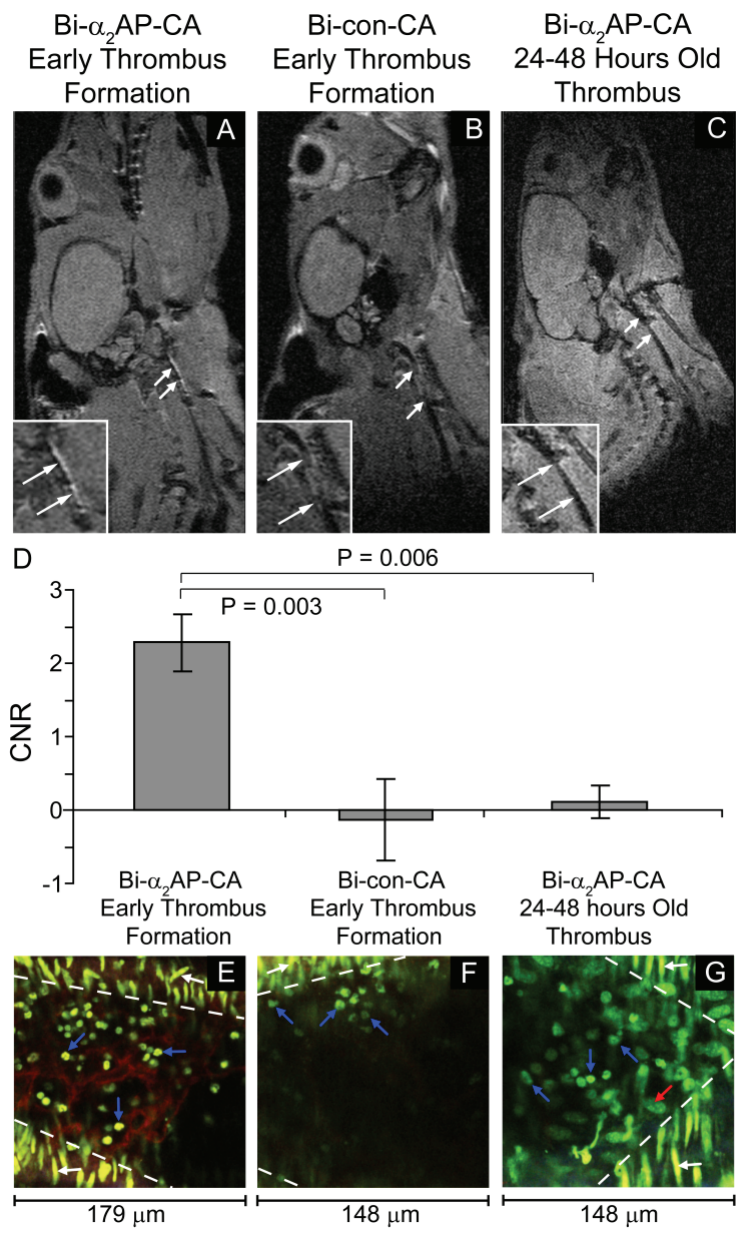

Chapter 6 - figure 6: In vivo magnetic resonance images (MRI) (A-C) and ex vivo two-photon laser-scanning microscopy (TPLSM) results (E-G) of early thrombus formation and 24-48 hour old thrombi in murine carotid arteries after injection of the bimodal $\alpha_{2}$-antiplasmin-based CA (Bi- $\left.\alpha_{2} \mathrm{AP}-\mathrm{CA}\right)(\mathrm{A}, \mathrm{C}, \mathrm{E}$, and $\mathrm{G}$ ) and the bimodal control CA (Bi-con-CA) (B and F). Contrast-to-noise ratios (CNR) are elevated in the early phases of thrombus formation after $B i-\alpha_{2} A P-C A$ administration (D). Panels E-G: Red: Bimodal CA. Green: Nuclear stain. Lumen is located between dotted lines. Blue arrows indicate leukocytes trapped in the thrombus. Elongated cells are smooth muscle cells (white arrows) and endothelial cells (red arrow). 
Chapter 9 